Florida International University

FIU Digital Commons

FIU Electronic Theses and Dissertations

University Graduate School

$10-26-2017$

\title{
Simplifying Sample Preparation using Fabric Phase Sorptive Extraction: Analysis of Trace Targeted Pollutant Residues in Environmental, Biological and Food Samples
}

Rodolfo Mesa

Florida International University, rmesa004@fiu.edu

DOI: $10.25148 /$ etd.FIDC004050

Follow this and additional works at: https://digitalcommons.fiu.edu/etd

Part of the Analytical Chemistry Commons, and the Water Resource Management Commons

\section{Recommended Citation}

Mesa, Rodolfo, "Simplifying Sample Preparation using Fabric Phase Sorptive Extraction: Analysis of Trace Targeted Pollutant Residues in Environmental, Biological and Food Samples" (2017). FIU Electronic Theses and Dissertations. 3513.

https://digitalcommons.fiu.edu/etd/3513 


\title{
FLORIDA INTERNATIONAL UNIVERSITY
}

\author{
Miami, Florida
}

\section{SIMPLIFYING SAMPLE PREPARATION USING FABRIC PHASE SORPTIVE EXTRACTION: ANALYSIS OF TRACE TARGETED POLLUTANT RESIDUES IN ENVIRONMENTAL, BIOLOGICAL AND FOOD SAMPLES}

A dissertation submitted in partial fulfillment of the

\author{
requirements for the degree of \\ DOCTOR OF PHILOSOPHY
}

in

CHEMISTRY

by

Rodolfo Mesa

2017 
To: Dean Michael R. Heithaus

College of Arts, Sciences and Education

This dissertation, written by Rodolfo Mesa, and entitled Simplifying Sample Preparation Using Fabric Phase Sorptive Extraction: Analysis of Trace Targeted Pollutant Residues in Environmental, Biological and Food Samples, having been approved in respect to style and intellectual content, is referred to you for judgment.

We have read this dissertation and recommend that it be approved.

Kathleen Rein

Anthony DeCaprio

\begin{tabular}{ll}
\hline Jeffrey Wells \\
\hline Abuzar Kabir
\end{tabular}

Kenneth G. Furton, Major Professor

Date of Defense: October 25, 2017

The dissertation of Rodolfo Mesa is approved.

Dean Michael R. Heithaus

College of Arts, Sciences and Education

Andrés G. Gil

Vice President for Research and Economic Development and Dean of the University Graduate School

Florida International University, 2017 
(C) Copyright 2017 by Rodolfo Mesa

All rights reserved. 


\section{DEDICATION}

Numerous wonderful people have positively contributed to helping me be successful in life. My mother and father, most notably, have sacrificed themselves throughout my lifetime to make me the best person I can be and ultimately allow me the opportunity to reach this goal of earning a Doctorate degree. It is ever present in my mind that their lives have been difficult in many instances but I want them to know that I recognize that because of their efforts mine has been much easier and that is, in my opinion, the true measure of success for parents. My brother Randy is one of the most extraordinary individuals I have ever met and he may not realize it but his perseverance has been an essential motivational force for me. If he had ever had the opportunity to read this he would have laughed at me and I love him for it. Perhaps at some point in my life I will have the mental fortitude to make the best of my life and live it like he lived his. The newest members of my immediate family are my wonderful love Lissette and the love of our lives, our precious daughter Miabella. Lissette is quite simply a benevolent individual who has made me consider views and aspects of situations that may not have otherwise and that has allowed me to become a more considerate and enduring man. My wonderful Miabella is now the foremost consideration in every decision-making thought I have and has brought a special joy to my life that I never imagined possible. I am extremely fortunate to have a family who have patiently loved me, even when I have made it very difficult for them to do so. I dedicate not only this work but my entire life to them. 


\section{ACKNOWLEDGMENTS}

I wish to thank the many professionals that have helped in my journey through my career at FIU, both undergraduate and graduate. I have had many professors and peers that have been a positive influence. Kenneth G. Furton, Ph.D. took time out of his very busy schedule to listen to me and welcome me into his research group when I was nothing more than an undergraduate chemistry student that was absolutely lost and subsequently, even with very limited interaction, managed to have a real significant influence in me in regards to how I conducted myself socially and professionally. Howard Holness, Ph.D. also helped me on several occasions with a variety of issues that I had in my career as a graduate student. He was always gracious and patient with me and others in the Furton Research Group, even when it was not part of his duties. My graduate committee, composed of Kathleen Rein, PhD., Anthony DeCaprio, PhD., Jeffrey Wells, PhD., and Abuzar Kabir, PhD. has given me very valuable advice and guidance regarding my research.

Lastly but definitely not least, I was fortunate to have lab colleagues that allowed me to feel comfortable working and made my time doing research enjoyable. Philip Russell Norman Davis is presently one of my closest friends and an amazing individual who I am quite fortunate to have known not only as a colleague but over time as a person. His ability to listen, understand and support me both professionally and personally has been truly invaluable. Vanquilla Shellman-Francis and Adhly Marie Huertas Rivera helped me tremendously on 
countless occasions. My experience in the lab would have been much more challenging had they not been so warm and accommodating.

I also worked directly with undergraduates who did their best to learn method development and validation as well as overarching principals of performing research while also being productive. Linda Maiben was an absolute pleasure to work with and in her short time in the Furton Research Group allowed me to meet my research objectives with greater efficiency. Jessica Jurmain was a great influence for me because not only was she very smart, hard-working and productive, she often challenged me to broaden my understanding and therefore be a better researcher. 


\section{ABSTRACT OF THE DISSERTATION}

\section{SIMPLIFYING SAMPLE PREPARATION USING FABRIC PHASE SORPTIVE EXTRACTION: ANALYSIS OF TRACE TARGETED POLLUTANT RESIDUES IN ENVIRONMENTAL, BIOLOGICAL AND FOOD SAMPLES}

by

Rodolfo Mesa

Florida International University, 2017

Miami, Florida

Professor Kenneth G. Furton, Major Professor

Sample preparation is an essential component of analytical methods in chemistry. It is not only necessary but also presents an opportunity to increase the effectiveness of the method significantly. There are various commercially available technologies for sample preparation, including numerous variations of LLE, SPE, and SPME. However, these technologies all present significant deficiencies, including the inability to extract directly from complex samples such as whole milk. Instrumental analysis has been improved greatly in the last two decades but still is not applicable to complex samples without sample preparation.

This work presents the theory of FPSE, including the synthesis of sol-gel sorbents, coating of FPSE cellulose substrates and the mechanism of retention. Original research data presented herein introduce a comprehensive view on 
possible applications of FPSE in forensic chemistry and otherwise. Five distinct FPSE-based methods were rigorously developed for analysis of targeted pollutant residues. These methods were validated and compare to leading methods published in peer-reviewed literature quite favorably. Four of the methods were coupled to HPLC-UV and designed for trace or ultra-trace analysis of PAHs, BTEX, substituted phenols and nitroaromatic explosives, respectively. An additional FPSE-based method was developed and validated for direct analysis of BPA and five estrogenic EDCs in commercially purchased whole milk. This latter was coupled to both HPLC-UV and HPLC(QQQ)-MS/MS.

The applicability of FPSE(PTHF) media was also tested for screening of aqueous samples and subsequent storage of analytes on the sorbent. My study included simultaneous extraction of a mixture of eight forensically significant compounds with various physicochemical properties and effective storage of each compound in frozen and ambient conditions for 32 weeks. These findings suggest that the storage ability of FPSE media can be extended as long as necessary, which is very significant in forensic laboratories where evidence often needs to be stored in a costly manner that may not be as effective in maintaining the chemical composition of the sample. 


\section{TABLE OF CONTENTS}

CHAPTER

PAGE

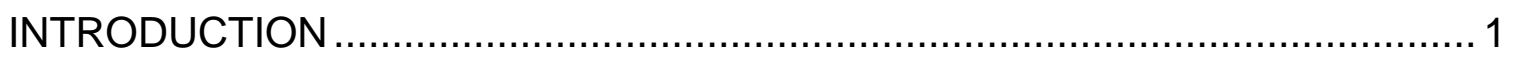

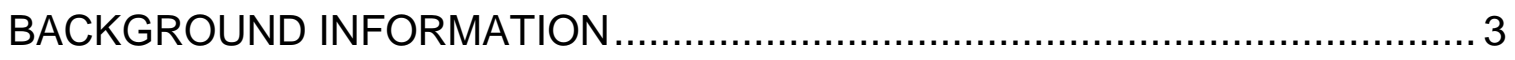

2.1 Sample Preparation in Analytical Chemistry .......................................... 3

2.2 Liquid-Liquid Extraction ................................................................ 7

2.2.1 Liquid-Liquid Microextraction........................................................ 14

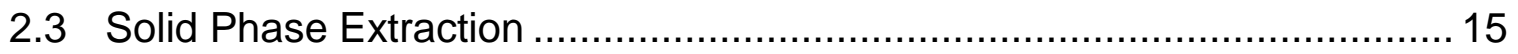

2.4 Solid Phase Microextraction ............................................................... 23

2.4.1 Stir-Bar Sorptive Extraction ......................................................... 29

2.4.2 Thin-Film Microextraction ............................................................. 30

2.5 Fabric Phase Sorptive Extraction ........................................................ 33

2.5.1 FPSE Theoretical Considerations ….................................................. 33

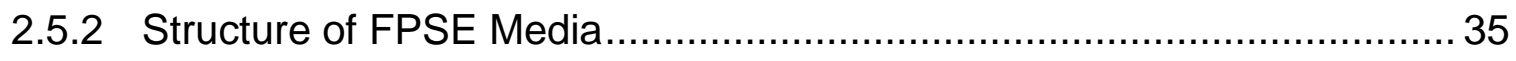

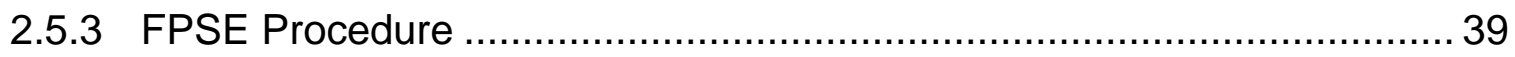

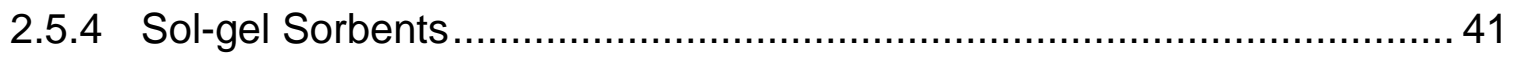

2.5.5 Advantageous Characteristics of FPSE …....................................... 43

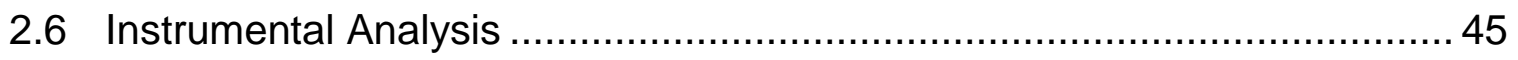

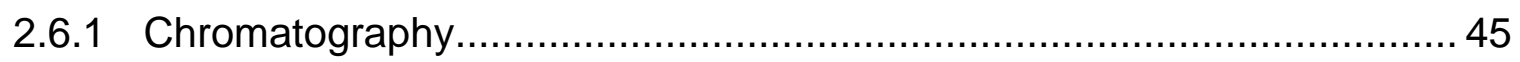

2.6.2 High Performance Liquid Chromatography ....................................... 48

2.6.3 Ultra-Violet/Visible Detector............................................................ 52

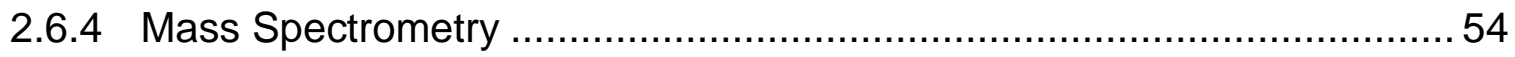

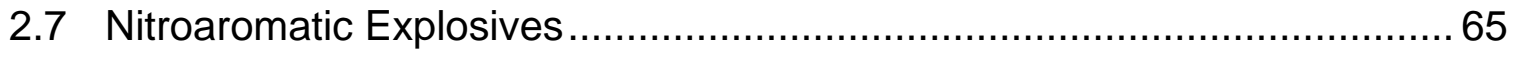

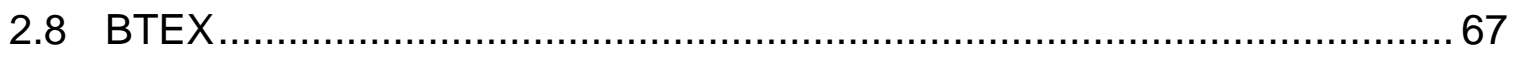




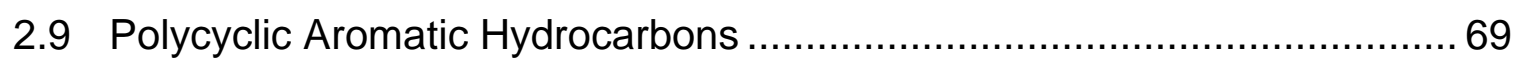

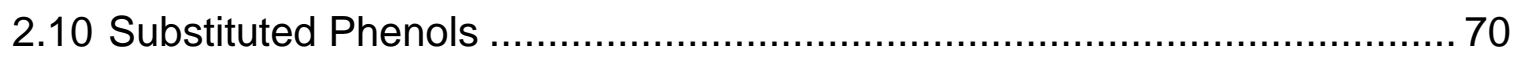

2.11 Endocrine Disrupting Chemicals in Milk ................................................. 72

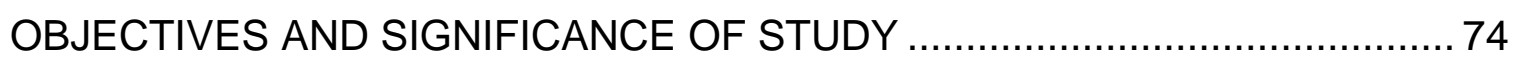

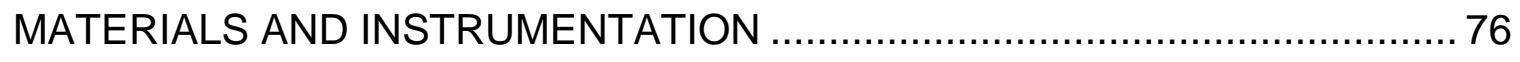

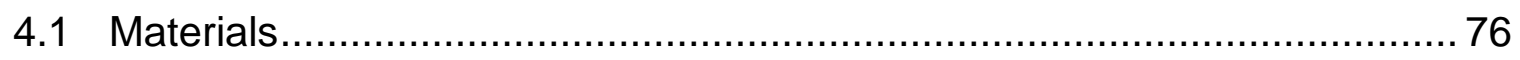

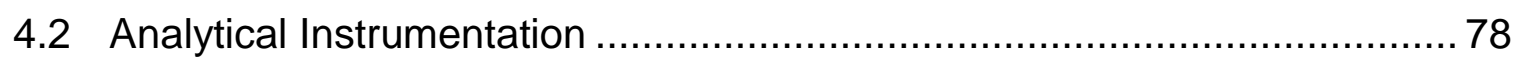

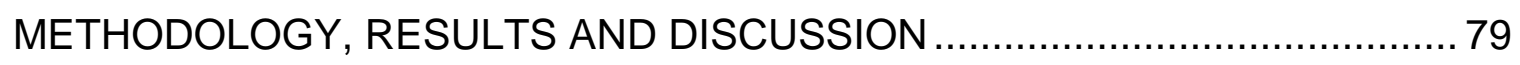

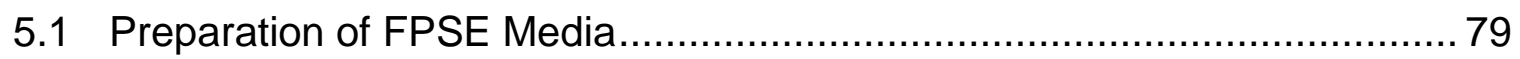

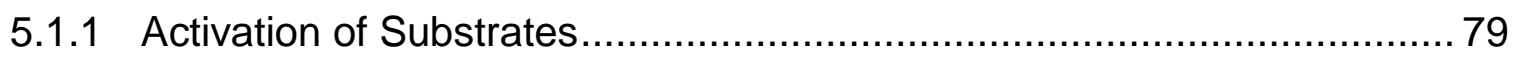

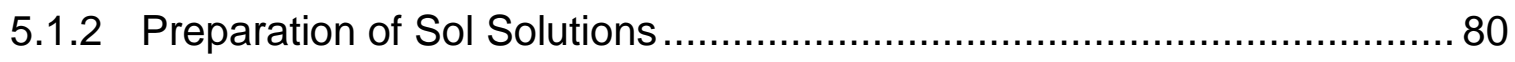

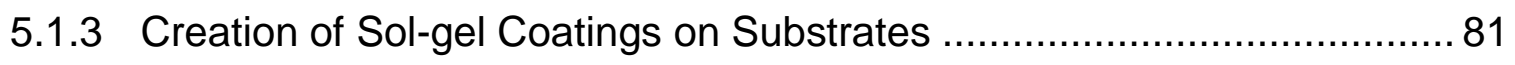

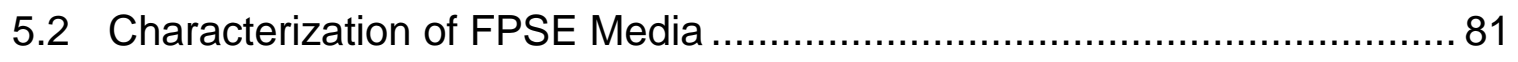

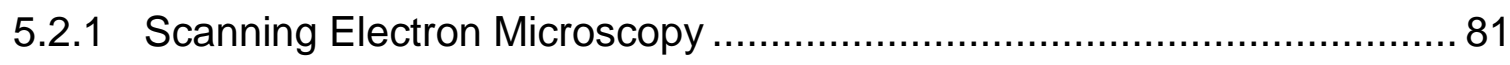

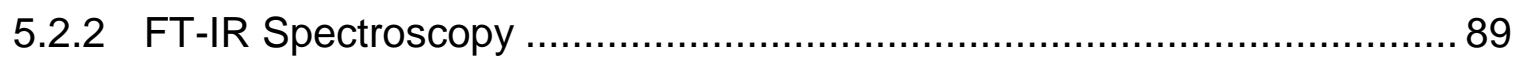

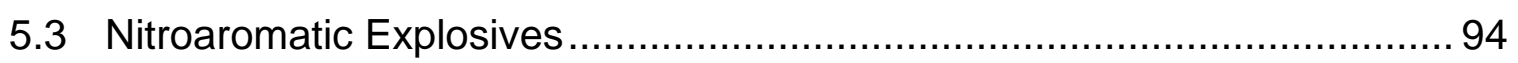

5.3.1 Preparation of Standard Solutions ....................................................... 95

5.3.2 Instrumental Method Development...................................................... 95

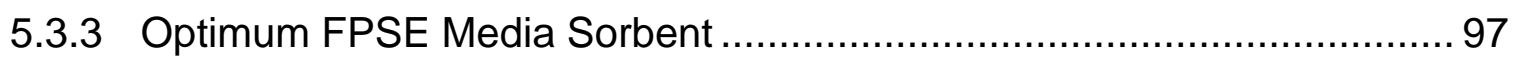

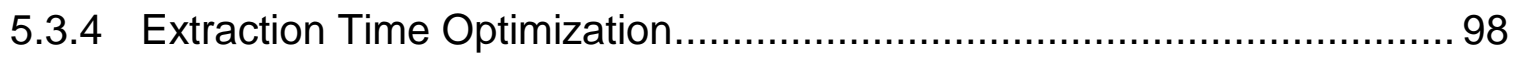

5.3.5 Back Extraction Time Optimization..................................................... 100

5.3.6 Extraction Volume Optimization ....................................................... 101

5.3.7 Linearity, Inter and Intraday Repeatability ......................................... 104

5.3.8 Limits of Detection and Quantitation............................................... 108 
5.3.9 Field Application to Environmental Samples ...................................... 110

5.4 Benzene, Toluene, Ethylbenzene, Xylenes ......................................... 112

5.4.1 Preparation of Standard Solutions..................................................... 113

5.4.2 Instrumental Method Development................................................. 113

5.4.3 Determination of Optimum FPSE Media Sorbent ............................... 114

5.4.4 Optimization of Sample Preparation Parameters ................................. 115

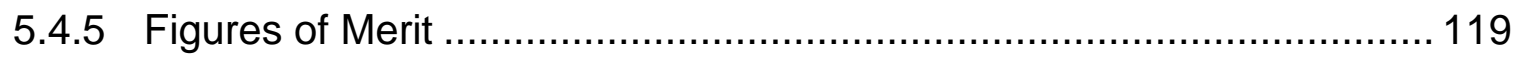

5.4.6 Application to Real Samples ........................................................ 122

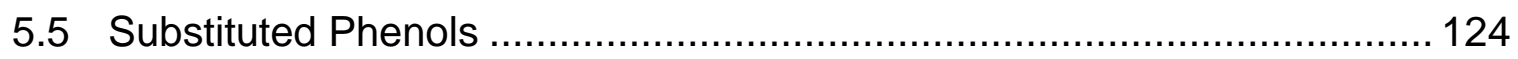

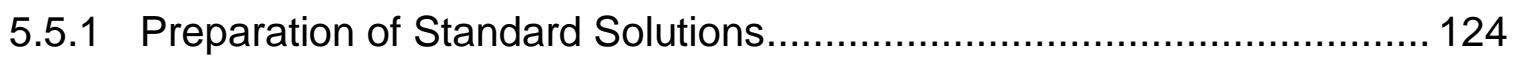

5.5.2 Instrumental Method Development.................................................. 125

5.5.3 Determination of Optimum FPSE Media Sorbent …............................. 126

5.5.4 Optimization of Sample Preparation Parameters ............................... 127

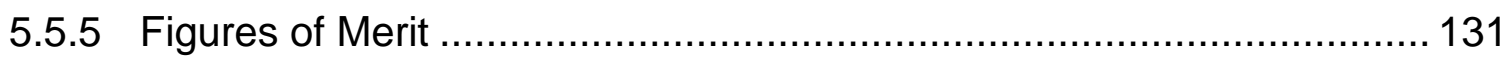

5.5.6 Field Application to Tap, Pond, and Reclaimed Water Samples ........... 136

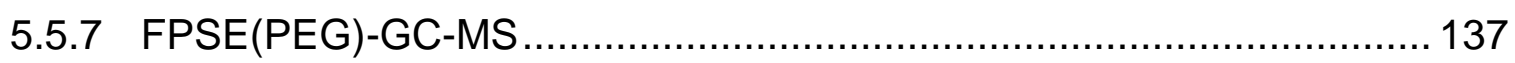

5.6 Polycyclic Aromatic Hydrocarbons …................................................ 138

5.6.1 Preparation of Standard Solutions........................................................ 139

5.6.2 Determination of Optimum FPSE Media Sorbent-............................. 140

5.6.3 Instrumental Method Development.................................................... 140

5.6.4 Optimization of Sample Preparation Parameters …............................ 141

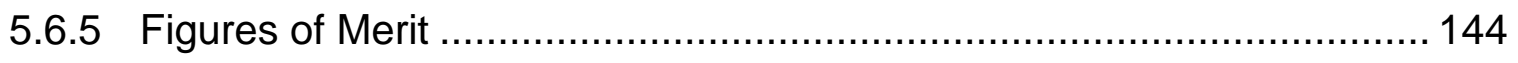

5.6.6 Ultra-Sensitive Alternate FPSE Procedure ........................................ 151

5.6.7 Field Application to Environmental Samples .................................... 152 
5.7 Endocrine Disrupting Chemicals in Milk ................................................ 154

5.7.1 Preparation of Standard Solutions................................................... 154

5.7.2 Instrumental Method Development.................................................... 155

5.7.3 Determination of Optimum FPSE Media Sorbent ............................... 156

5.7.4 Optimization of Sample Preparation Parameters ............................... 157

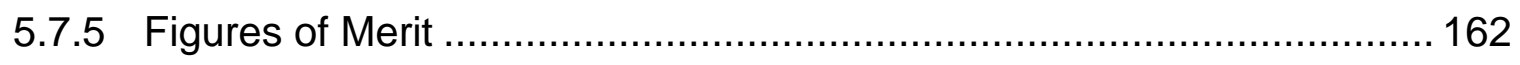

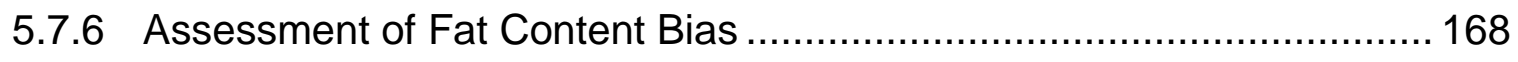

5.7.7 Direct Analysis vs. Analysis After Protein Precipitation and Defatting ... 169

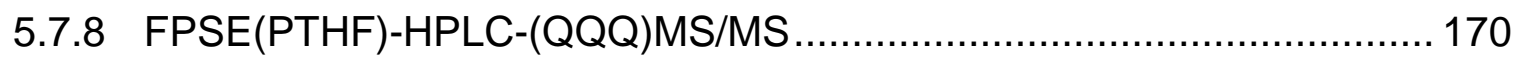

5.7.9 Comparison of Procedure of Developed Method to SBSE and SPE

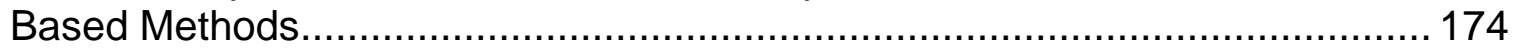

5.7.10 Application of Developed Method to Spiked Urine and Whole Blood

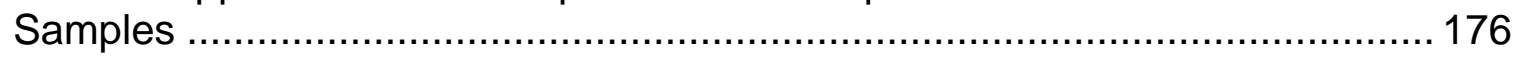

5.8 FPSE Media: Highly Effective Extraction and Storage Device ................ 177

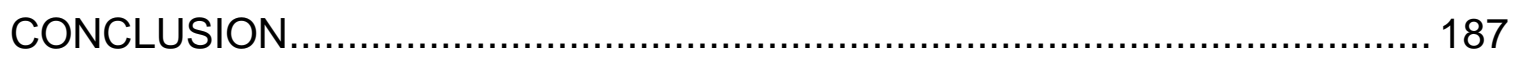

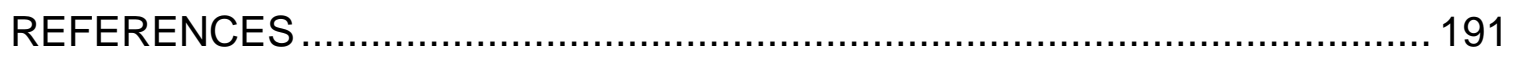

VITA 


\section{LIST OF TABLES}

TABLE

PAGE

Table 1: List of the ten nitroaromatic explosive compounds included in this report, including their structure with the corresponding octanol-water partition coefficient (log Kow) available, it is given.

Table 2: List of the 5 BTEX compounds included in this report, in addition to the structure and octanol-water partition coefficient (log $\mathrm{K}_{\mathrm{ow}}$ ) of each.

Table 3: List of the 5 PAHs included in this report, including the structure and octanol-water partition coefficient (log Kow) of each.

Table 4: List of the 5 substituted phenols included in this report, including the structure and octanol-water coefficient (log Kow) of each.

Table 5: Structure, source of prduction and octanol-water partition coefficient (log Kow) of each endocrine disruptor included in this study.

Table 6: Summary of the compound specific results for intra and inter-day repeatability as well as dynamic range and the corresponding correlation coefficient for the developed FPSE(PEG)-HPLC-UV method for the analysis of nitroaromatic explosives in water

Table 7: Compound specific results for experimental calculations of LOD and LOQ for the developed FPSE(PEG)-HPLC-UV method and comparison to performance of methods in the literature. N/A signifies that the compound was not included in the research article.

Table 8: Summary of the compound specific results for intra and inter-day repeatability as well as dynamic range and the corresponding correlation coefficient for the developed FPSE(PTHF)-HPLC-UV method for analysis of BTEX compounds in aqueous samples.

Table 9: Compound specific results for experimental calculations of LOD and LOQ for the developed FPSE(PEG)-HPLC-UV method and comparison to performance of a method in the literature.

Table 10: Summary of the compound specific results for intra and inter-day repeatability as well as dunamic range and the corresponding correlation coefficient for the developed FPSE(PEG)-HPLC-UV method for analysis of substituted phenols in aqueous samples. 
Table 11: Compound specific results for experimental calculations of LOD and LOQ for the developed FPSE(PEG)-HPLC-UV method and comparison performance of a method in the literature.

Table 12: Summary of the compound specific results for intra and inter-day repeatability as well as dynamic range and the corresponding correlation coefficient for the developed FPSE(PDMDPS)-HPLC-UV method for analysis of PAHs in aqueous samples.

Table 13: Compound specific results for experimental calculations of LOD and LOQ for the developed FPSE(PEG)-HPLC-UV method and cmparison to performance of a method in the literature.

Table 14: Comparison of the LOD range for the developed FPSE(PEG)HPLC-UV method to that of eight methods in the literature. The ranges reported for each method correspond to several PAHs, not necessarily the same included in the research presented herein.

Table 15: Summary of the compound specific results for intra and inter-day repeatability as well as dynamic range and the corresponding correlation coefficient for the developed FPSE(PTHF)-HPLC-UV method for analysis of EDCs in whole milk.

Table 16: Comparison of LOD and LOQ values obtained with the developed FPSE(PTHF)-HPLC-UV method vs HF-LPME-HPLC-DAD/FD and Acetate$\mathrm{MeOH}-S P E-H P L C-D A D$ methods from the literature. N/A signifies that the compound is not included in the data.

Table 17: Comparison of LOD and LOQ values determined with the developed FPSE(PTHF)-HPLC-UV and FPSE(PTHF)-HPLC-(QQQ)MS/MS methods and an ACN-SPE-HPLC-MS/MS method from the literature. N/A: that compound not included in the data [118]. 


\section{LIST OF FIGURES}

FIGURE

PAGE

Figure 1: Separatory funnel showing two distinct layers of immiscible liquid, one aqueous and one organic. 8

Figure 2: Demonstration of proper procedure for venting a separatory funnel. ....9

Figure 3: LLE scheme where the partitioning of analytes depends solely on $\mathrm{KD}_{\mathrm{D}}[18]$.

Figure 4: LLE scheme for a weak acid, where the partitioning of analytes depends on $K_{D}$ but also the relative abundance of the protonated acid HA, given by the acid dissociation constant Ka [18].

Figure 5: Representative plot of extraction efficiency vs number of extractions in LLE [18].

Figure 6: Plot of extraction efficiency vs $\mathrm{pH}$ of solution for a weak acid HA [18]. 13

Figure 7: Image of various commercially available SPE cartridges including plastic and glass construction [33].

Figure 8: Schematic of composition of SPE device employing disk technology instead of a packed cartridge [36].

Figure 9: Guide for stationary phase selection for organic compounds from aqueous and organic matrices. SAX, strong anion exchanger; SCX, strong cation exchanger; WCX, weak cation exchanger; RP, reversed-phase sampling conditions; NP, normal-phase sampling conditions; IE, ionexchange sampling conditions.

Figure 10: Graphical representation of curve used to determine breakthrough volume. VB stands for breakthrough volume; VR for the chromatographic elution volume; $\mathrm{VC}$ for the sample volume corresponding to the isolation of the maximum amount of analytes; $\mathrm{C} 0$ for the concentration of analyte in the sample; $\sigma \vee$ for the standard deviation of the derivative curve for the plot.

Figure 11: Top: Original schematic of SPME apparatus construction. Bottom: Schematic of a commercially available SPME fiber with exposure apparatus.... 25

Figure 12: Plot of relationship between extracted amount and extraction time for SPME, including the linear, kinetic, and equilibrium regimes. 28 
Figure 13: Schematic of the composition of TFME apparatus and steps involved in the corresponding headspace analysis procedure. 1. deactivated stainless steel rod; 2 . flat sheet membrane; 3 . sample solution; 4. tefloncoated stirring bar; 5. membrane; 6 . injector nut; 7. membrane; 8. glass liner; 9. capillary column

Figure 14: Schematic summarizing traditional SPME, its major derivatives and some distinguishing characteristics [14].

Figure 15: Picture of three $5 \mathrm{~cm}^{2}$ sol-gel PTHF FPSE media. The substrate is cellulose (cotton).

Figure 16: Representation of the chemical structure of the surface of cellulose, showing three exposed hydroxyl groups at positions 2, 3 and 6 of each glucopyranose unit.

Figure 17: Schematic of sol-gel PDMS FPSE media. The sol-gel network is enclosed in red.

Figure 18: Schematic of sol-gel PDMDPS FPSE media. The sol-gel network is enclosed in red.

Figure 19: Schematic of sol-gel PTHF FPSE media. The sol-gel network is enclosed in red.

Figure 20: Schematic of sol-gel PEG FPSE media. The sol-gel network is enclosed in red.

Figure 21: Picture sequence outlining procedure of sample preparation by FPSE.

Figure 22: Schematic of the major steps in the process of sol-gel synthesis and bonding to a surface, forming a coating that serves as a sorbent.

Figure 23: SEM image showing the high porosity inherent in hybrid inorganicorganic sol-gel materials [70].

Figure 24: Classification of chromatographic systems by the mobile phase....... 47

Figure 25: Typical HPLC chromatogram. Each peak represents a specific compound and each compound has a characteristic retention time (time at which the peak is seen from the start of the chromatogram).

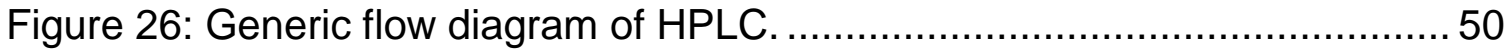

Figure 27: General schematic of generic UV detector..................................... 53 
Figure 28: Diagram of generic mass spectrometer.

Figure 29: Diagram of ESI source with skimmers for ion focalization and heated nitrogen gas for desolvation.

Figure 30: Diagram of ESI source with heated capillary for desolvation.

Figure 31: Illustration of the effect of the magnitude of applied voltage on a drop of liquid in ESI. When the voltage is low (left) the drop is spherical, at a higher voltage it elongates (middle) until the pressure is greater than the surface tension (at the onset voltage) and smaller droplets are released. 58

Figure 32: Two situations where the concept of looking at resolution per the height of the valley between the two peaks is illustrated.

Figure 33: Schematic of a quadrupole mass spectrometer, including the path of an ion transmitted to the detector (resonant ion) and another that is expelled before reaching the detector (non-resonant ion).

Figure 34: Low pass mass range representative diagram. ............................. 62

Figure 35: High pass mass range representative diagram............................. 62

Figure 36: Narrow mass range representative diagram.................................. 63

Figure 37: Picture of state-of-the-art QQQ mass spectrometer........................65

Figure 38: Labeled diagram of a scanning electron microscope......................... 82

Figure 39: Labeled schematic of the interaction between electrons from the electron beam and the sample in SEM.

Figure 40: Cross-sectional 100x magnified BSE generated SEM image of uncoated cellulose fabric (top left); sol-gel MTMS coated cellulose fabric (top right); PTHF FPSE media on cellulose (bottom left); PEG FPSE media on cellulose (bottom right).

Figure 41: 100x magnified BSE generated SEM surface view of uncoated cellulose fabric (top left); sol-gel MTMS coated cellulose fabric (top right); PTHF FPSE media on cellulose (bottom left); PEG FPSE media on cellulose (bottom right).

Figure 42: 500x magnified BSE generated SEM surface view of uncoated cellulose fabric (top left); sol-gel MTMS coated cellulose fabric (top right); PTHF FPSE media on cellulose (bottom left); PEG FPSE media on cellulose (bottom right). 
Figure 43: Cross-sectional 100x magnified BSE generated SEM image of uncoated polyester fabric (left); PDMDPS FPSE media (right).

Figure 44: 100x magnified BSE generated SEM surface view of uncoated polyester fabric (left); PDMDPS FPSE media (right).

Figure 45: 500x magnified BSE generated SEM surface view of uncoated polyester fabric (left); PDMDPS FPSE media (right).

Figure 46: The top FT-IR spectrum is of uncoated cellulose fabric, the one at the bottom is of PTHF FPSE media on cellulose.

Figure 47: The top FT-IR spectrum is of uncoated cellulose fabric, the one at the bottom is of PEG FPSE media on cellulose.

Figure 48: The top FT-IR spectrum is of uncoated polyester fabric, the one at the bottom is of PDMDPS FPSE on polyester.

Figure 49: FT-IR spectrum of pure PDMDPS polymer.

Figure 50: Structure of stationary phase of Ascentis RP Amide HPLC column.

Figure 51: Representative chromatogram of developed method for 10 nitroaromatic explosives.

Figure 52: Experimental data of extraction time optimization for FPSE(PEG)HPLC-UV analysis of nitroaromatic explosives in water.

Figure 53: Experimental data of back extraction time optimization for FPSE(PEG)-HPLC-UV analysis of nitroaromatic explosives in water.

Figure 54: Experimental data of back extraction solvent optimization for FPSE(PEG)-HPLC-UV analysis of nitroaromatic explosives in water.

Figure 55: Experimental data of back extraction volume optimization for FPSE(PEG)-HPLC-UV analysis of nitroaromatic explosives in water. 102

Figure 56: Experimental data for determination of compound specific linear range for the developed FPSE(PEG)-HPLC-UV method for analysis of nitroaromatic explosives in water.

Figure 57: Schematic demonstrating how peak-to-peak noise distances are measured 
Figure 58: Experimental data pertaining to the comparison of the effectiveness of the developed FPSE(PEG)-HPLC-UV method at an FIU pond vs. analysis of the same sample transported to the laboratory.

Figure 59: Experimental data pertaining to the comparison of the effectiveness of the developed FPSE(PEG)-HPLC-UV method at Key Biscayne Beach vs. analysis of the same sample transported to the laboratory

Figure 60: Representative chromatogram of analysis of a $10 \mathrm{ppb}$ solution of the 5 pertinent BTEX compounds from deionized water with the developed FPSE-HPLC-UV method.

Figure 61: Experimental data of the comparison of the effectiveness of FPSE-PTHF and FPSE-PDMDPS media, respectively, for extraction of BTEX compounds from aqueous matrices.

Figure 62: Experimental data of extraction time optimization of the FPSE(PTHF)-HPLC-UV being developed for analysis of BTEX compounds in aqueous samples.

Figure 63: Experimental data of back extraction time optimization of the FPSE(PTHF)-HPLC-UV being developed for analysis of BTEX compounds in aqueous samples.

Figure 64: Experimental data of back extraction solvent optimization of the FPSE(PTHF)-HPLC-UV being developed for analysis of BTEX compounds in aqueous samples.

Figure 65: Experimental data of extraction volume optimization of the FPSE(PTHF)-HPLC-UV being developed for analysis of BTEX compounds in aqueous samples.

Figure 66: Experimental data of the linearity study for the developed FPSE(PTHF)-HPLC-UV method for analysis of BTEX compounds in aqueous samples.

Figure 67: Experimental data showing results of the application of the developed FPSE(PEG)-HPLC-UV method to spiked samples of tap, pond, and reclaimed water in the form of relative recovery \%. The samples were spiked to a concentration of $10 \mathrm{ppb}$ per analyte.

Figure 68: Representative HPLC-UV chromatogram of an analysis of 5 substituted phenols from deionized water. The elution order from the C-18 Extend column is as follows: (1) 4-chlorophenol, (2) 3,5-dimethyl phenol, (3) 2,6-dichlorophenol, (4) 2,4,6-trichlorophenol, (5) 2,4-diisopropyl phenol. ... 126 
Figure 69: Experimental data of the comparison of the effectiveness of FPSE-PTHF, FPSE-PEG, and FPSE-PEG/PEO media, respectively, for extraction of substituted phenols from aqueous matrices.

Figure 70: Experimental data of extraction time optimization of the FPSE(PEG)-HPLC-UV method being developed for analysis of substituted phenols in aqueous samples.

Figure 71: Experimental data of back extraction time optimization of the FPSE(PEG)-HPLC-UV method being developed for analysis of substituted phenols in aqueous samples.

Figure 72: Experimental data of extraction sample volume optimization of the FPSE(PEG)-HPLC-UV method being developed for analysis of substituted phenols in aqueous samples.

Figure 73: Experimental data of linearity study of the FPSE(PEG)-HPLC-UV method developed for analysis of substituted phenols in aqueous samples. ... 132

Figure 74: Comparison of response magnitudes and triplicate set repeatability for analysis of deionized, tap, pond, and reclaimed water samples spiked with five substituted phenols. 136

Figure 75: Representative GC-MS chromatogram of an analysis of 5 substituted phenols from deionized water. The elution order from the GC column is as follows: (1) 3,5-dimethyl phenol, (2) 4-chlorophenol, (3) 2,6dichlorophenol, (4) 2,4,6-trichlorophenol, (5) 2,4-diisopropyl phenol.

Figure 76: Representative HPLC-UV chromatogram of an analysis of 5 PAHs from deionized water. Each peak is labeled with the identity of the corresponding compound.

Figure 77: Experimental data of extraction time optimization of the FPSE(PDMDPS)-HPLC-UV method being developed for analysis of PAHs in aqueous samples.

Figure 78: Experimental data of back extraction time optimization of the FPSE(PDMDPS)-HPLC-UV method being developed for analysis of PAHs in aqueous samples.

Figure 79: Experimental data of extraction sample volume optimization of the FPSE(PDMDPS)-HPLC-UV method being developed for analysis of $\mathrm{PAHs}$ in a aqueous samples.

Figure 80: Experimental data of linearity study of the FPSE(PDMDPS)HPLC-UV method developed for analysis of PAHs in aqueous samples. 
Figure 81: Apparatus used to coat and evaluate the ST-SPME disk:

(1) PEEK tube, (2) PEEK screw, (3) PEEK ferrule, (4) stainless steel nut,

(5) PEEK ferrule, (6) sintered titanium disk, and (7) stainless steel screw.

Figure 82: Compound specific relative recovery percentages of each of the five PAHs from pond, reclaimed, and saline water samples, respectively.

Figure 83: Representative HPLC-UV chromatogram of an FPSE extraction of the 6 EDCs from whole milk. The concentration of BPA, E2, and E1 in the milk was $100 \mathrm{ppb}$, the concentration of EE2, HEX, and DES was $200 \mathrm{ppb}$, and the injection volume was $15 \mu \mathrm{L}$

Figure 84: Experimental data of the comparison of the effectiveness of FPSE-PTHF and FPSE-PDMS media, respectively, for extraction of EDCs from whole milk.

Figure 85: Experimental data of extraction time optimization of the FPSE(PTHF)-HPLC-UV method being developed for analysis of 6 EDCs from whole milk.

Figure 86: Experimental data of back extraction time optimization of the FPSE(PTHF)-HPLC-UV method being developed for analysis of 6 EDCs from whole milk.

Figure 87: Experimental data of extraction volume optimization of the FPSE(PTHF)-HPLC-UV method being developed for analysis of 6 EDCs from whole milk.

Figure 88: Experimental results of stirring speed optimization of the FPSE(PTHF)-HPLC-UV method being developed for analysis of 6 EDCs from whole milk.

Figure 89: Results of sorbent loading capacity study of the developed FPSE(PTHF)-HPLC-UV method for analysis of 6 EDCs from whole milk.

Figure 90: Illustration of experimental data comparison responses generated by analysis of EDCs in whole milk by the developed FPSE(PTHF)-HPLC-UV method before and after solvent evaporation and analyte reconstitution. 168

Figure 91: Experimental data of assessment of fat content effect on extraction sensitivity and reliability. Extraction efficiency of the developed FPSE(PTHF)-HPLC-UV method is explored for whole milk, reduced fat milks $(2 \%$ and $1 \%)$ and fat free milk $(<0.1 \%)$, respectively. 
Figure 92: Graphical representation of analysis bias due to protein precipitation and defatting using the developed FPSE(PTHF)-HPLC-UV for analysis of 6 EDCs from whole milk.

Figure 93: EICs of FPSE based analysis of whole milk spiked with the 6 pertinent EDCs. The concentration for BPA, E2, E1, DES, and HEX was $10 \mathrm{ppb}$ of each in whole milk. The concentration of EE2 was $50 \mathrm{ppb}$.

Figure 94: Flowcharts comparing the procedures of two recently published methods (SBSE and SPE used for sample preparation, respectively) for analysis of milk to the one being introduced here.

Figure 95: Chromatogram obtained from application of developed FPSE(PTHF)-HPLC-UV method to spiked diluted whole blood. The peaks correspond to the EDCs as follows: (1) BPA, (2) E2, (3) EE2, (4) D1, (5) DES, (6) HEX

Figure 96: Chromatogram obtained from application of developed FPSE(PTHF)-HPLC-UV method to spiked urine. The peaks correspond to the EDCs as follows: (1) BPA, (2) E2, (3) EE2, (4) D1, (5) DES, (6) HEX.

Figure 97: Experimental data showing compound specific results of the same day analysis of 8 relevant compounds in deionized water with the developed FPSE(PTHF)-HPLC-UV method vs the responses after frozen storage in the FPSE media sorbent for 2, 4, 6, 8, 16, and 32 weeks.

Figure 98: Experimental data showing compound specific results of the same day analysis of 8 relevant compounds in deionized water with the developed FPSE(PTHF)-HPLC-UV method vs the responses after storage at ambient temperature in the FPSE media sorbent for 2, 4, 6, 8, 16, and 32 weeks.

Figure 99: Experimental data showing compound specific results of the same day analysis of a spiked sample of pond water with the developed FPSE(PTHF)-HPLC-UV method vs the responses after refrigerated storage of the solution for $1,2,3,14$, and 32 weeks.

Figure 100: Experimental data showing compound specific results of the same day analysis of a spiked sample of pond water with the developed FPSE(PTHF)-HPLC-UV method vs the responses after storage of the solution at ambient temperature for $1,2,3,14$, and 32 weeks. 184

Figure 101: Experimental data showing results of analysis of 8 relevant compounds in deionized water vs the responses after refrigerated storage in the FPSE media sorbent for 1, 2, 4, 8, 16, and 32 weeks. Uncoated cellulose was used instead of FPSE-PTHF media. 
Figure 102: Experimental data showing results of analysis of 8 relevant compounds in deionized water vs the responses after storage at ambient temperature in the FPSE media sorbent for 2, 4, 6, 8, 16, and 32 weeks. Uncoated cellulose was used instead of FPSE-PTHF media. 


\section{LIST OF ABBREVIATIONS}

$\begin{array}{ll}\text { \% RSD } & \text { Percent Relative Standard Deviation } \\ \mu \mathrm{ESI} & \text { Micro Electrospray lonization } \\ \text { 1,3-DNB } & \text { 1,3-Dinitrobenzene } \\ \text { 2,4-DIPP } & \text { 2,4-Diisopropylphenol } \\ \text { 2,6-DCP } & \text { 2,6-Dichlorophenol } \\ \text { 2,6-DNT } & \text { 2,6-Dinitrotoluene } \\ \text { 2-NDPA } & \text { 2-Nitrodiphenylamine } \\ \text { 2-NT } & \text { 2-Nitrotoluene } \\ \text { 3,4-DMP } & \text { 3,4-Dimethylphenol } \\ \text { 3,5-DMP } & \text { 3,5-Dimethylphenol } \\ \text { 3-NT } & \text { 3-Nitrotoluene } \\ \text { 4-CP } & \text { 4-Chlorophenol } \\ \text { 4-NT } & \text { Alternating Current } \\ \text { AC } & \text { Acetonitrile } \\ \text { ACN } & \text { 4itrotoluene }\end{array}$
ALS
Automated Liquid Sampler 


\begin{tabular}{|c|c|}
\hline BPA & Bisphenol A \\
\hline BSE & Backscattered Electrons \\
\hline BTEX & Benzene, Toluene, Ethylbenzene, Xylenes \\
\hline CID & Collision Induced Dissociation \\
\hline CME & Capillary Microextraction \\
\hline$D-\mu S P E-U P L C$ & $\begin{array}{l}\text { Dispersive Micro Solid Phase Extraction- } \\
\text { Ultra Performance Liquid Chromatography }\end{array}$ \\
\hline DAD & Diode Array Detector \\
\hline DC & Direct Current \\
\hline DES & Diethylstilbestrol \\
\hline DLLME & Dispersive Liquid-Liquid Microextraction \\
\hline DPA & Diphenylamine \\
\hline $\mathrm{E} 1$ & Estrone \\
\hline E2 & Estradiol \\
\hline EDC & Endocrine Disrupting Chemical \\
\hline EE2 & Ethilnyl Estradiol \\
\hline ESI & Electrospray Ionization \\
\hline FID & Flame Ionization Detector \\
\hline
\end{tabular}




$\begin{array}{ll}\text { FPSE } & \text { Fabric Phase Sorptive Extraction } \\ \text { FT-IR } & \text { Fourier Transform-Infrared Spectroscopy } \\ \text { GAC } & \text { Green Analytical Chemistry } \\ \text { GC } & \text { Gas Chromatography } \\ \text { GC-MS } & \text { Gas Chromatography Mass Spectrometry } \\ \text { HEX } & \text { Hexestrol } \\ \text { HF-LPME } & \text { Hollow Fiber-Liquid Phase Microextraction } \\ \text { HPLC } & \text { High Performance Liquid Chromatography } \\ \text { HPLC-MS/MS } & \begin{array}{l}\text { High Performance Liquid Chromatography- } \\ \text { Tandem Mass Spectrometry Detector } \\ \text { High Performance Liquid Chromatography- } \\ \text { HPLC-UV }\end{array} \\ \text { Ultra Violet Detector } \\ \text { HS }\end{array}$




\begin{tabular}{|c|c|}
\hline LLME & Liquid-Liquid Microextraction \\
\hline LOD & Limit of Detection \\
\hline LOQ & Limit of Quantitration \\
\hline ME & Microextraction \\
\hline $\mathrm{MeOH}$ & Methanol \\
\hline MQL & Method Quantitation Limit \\
\hline MRM & Multiple Reaction Monitoring \\
\hline MS & Mass Spectrometry \\
\hline MTMS & Methyltrimethoxysiloxane \\
\hline$N$ & Chromatographic Efficiency \\
\hline nESI & Nano Electrospray lonization \\
\hline NB & Nitrobenzene \\
\hline NHANES & $\begin{array}{l}\text { National Health and Nutrition Examination } \\
\text { Survey }\end{array}$ \\
\hline NP & Normal Phase \\
\hline ODS & Ovarian Dysgenesis Syndrome \\
\hline $\mathrm{PAH}$ & Polycyclic Aromatic Hydrocarbon \\
\hline PCF & Preconcentration Factor \\
\hline
\end{tabular}




\begin{tabular}{|c|c|}
\hline PCSA & Primary Contact Surface Area \\
\hline PDMDPS & Polydimethyl Diphenyl Siloxane \\
\hline PDMS & Polydimethylsiloxane \\
\hline PEG & Polyethylene Glycol \\
\hline PEG/PEO & $\begin{array}{l}\text { Polyethylene Glycol Polyethylene Oxide } \\
\text { Copolymer }\end{array}$ \\
\hline PEO & Polyethylene Oxide \\
\hline ppb & Parts per Billion \\
\hline ppm & Parts per Million \\
\hline ppt & Parts per Trillion \\
\hline PTFE & Polytetrafluoroethylene \\
\hline PTHF & Poly-tetrahydrofuran \\
\hline$Q Q Q$ & Triple Quadrupole Mass Spectrometer \\
\hline QTRAP & Quadrupole Ion Trap Mass Spectrometer \\
\hline RP & Reverse Phase \\
\hline Rs & Chromatographic Resolution \\
\hline$S / N$ & Signal-to-Noise Ratio \\
\hline SAX & Strong Anion Exchanger \\
\hline
\end{tabular}




$\begin{array}{ll}\text { SBSE } & \text { Stir-Bar Sorptive Extraction } \\ \text { SCX } & \text { Strong Cation Exchange } \\ \text { SE } & \text { Secondary Electrons } \\ \text { SEM } & \text { Scanning Electron Microscope } \\ \text { SPE } & \text { Solid Phase Extraction } \\ \text { SPME } & \text { Solid Phase Microextraction } \\ \text { TCP } & \text { Trichlorophenol } \\ \text { TFA } & \text { Trifluoro Acetic Acid } \\ \text { TFME } & \text { Thin Film Microextraction } \\ \text { TNB } & \text { Trinitrobenzene } \\ \text { TNT } & \text { Trinitrotoluene } \\ \text { VWD } & \text { Weak Cation Exchange } \\ \text { US EPA } & \text { Anited States Environmental Protection } \\ \text { UV } & \text { Ultra Violet } \\ \text { Variable Wavelength Detector }\end{array}$




\section{INTRODUCTION}

It is an unfortunate reality, to be sure, that human beings are exposed to numerous dangers in every-day life. In some instances, these dangers are known and avoided with relative ease, but this is not always the case. Some of these hidden dangers are a result of trace or ultra-trace level concentrations of toxic compounds in environmental waters or nutrition sources.

There are several ways, for example, in which nitroaromatic explosive compounds can accumulate in environmental waters; bodies of water close to sites of former ammunition plants are likely to have explosive compounds in them [1] and trace levels of contaminants can result indirectly from military exercises and lead to unexploded ordinances being released into oceans [2-4]. These contaminants in environmental waters can be hazardous to wildlife in these areas, and ultimately to humans even at concentrations in the range of low $\mathrm{ng} / \mathrm{mL}$ in drinking water [5].

Benzene, Toluene, Ethylbenzene and Xylene isomers (BTEX) and Polycyclic Aromatic Hydrocarbons (PAHs) are an even more prevalent threat to the health of human beings as environmental pollutants than nitroaromatic explosives because of their carcinogenic nature. All BTEX compounds are known to be in the environment and will continue to be because their sources of production include power plants and all engines that run on gasoline or petroleum $[6,7]$. In the Fourth National Report on Human Exposure to Environmental Chemicals, ten different PAH metabolites were measured in the urine of more than 2,504 
participants. The participants, all of whom took part in the National Health and Nutrition Examination Survey (NHANES), were a minimum of 6 years old. The quantity of PAH metabolites was measured in urine in order to estimate the amounts of PAHs that had entered the bodies of the participants. The results clearly indicate widespread presence of these harmful chemical class of compounds in the United States because metabolites were found in most of the urine samples tested [8].

Substituted phenols, produced in wood processing plants, pharmaceutical labs and oil refineries, [9] among other kinds of common places, are also toxic compounds that can cause severely damaging or fatal damage [10]. These compounds can affect the viability of hemoglobin [9] and the urinary, circulatory, respiratory and central nervous systems of human beings [11].

Endocrine Disrupting Chemicals (EDCs) are very worrisome emerging contaminants not only in the environment but also food sources such as milk and other dairy products. There are many compounds that are considered EDCs, including bisphenol A (BPA) and a plethora of synthetic and natural hormones. As it pertains to female reproductive health, for example, the effects of EDC exposure are given by an umbrella term, Ovarian Dysgenesis Syndrome (ODS), which includes many troubling problems including abnormal Mullerian duct development, abnormal ovaries and reproductive tract cancer [12]. Additionally, maternal exposure to EDCs can result in decreased placental function because of inhibition of trophoblast formation leading to lack of reproductive success in 
female mammals [12]. Bisphenol A by itself is an increasingly significant problem in the environment and food items because millions of tons are produced annually worldwide and it is a persistent pollutant [13].

Fabric phase sorptive extraction (FPSE) is a novel sample preparation technique developed by Kabir and Furton at Florida International University [14]. Fabric Phase Sorptive Extraction is presented herein for development, validation, and application of methods for direct immersion trace analysis of nitroaromatic explosives, PAHs, and EDCs from various environmental waters. An FPSEbased method was also developed and validated for trace analysis of EDCs from whole milk, a primary food source worldwide for infants. The developed methods were found to outperform those considered the gold standard in their respective fields, presenting a paradigm shift in the hierarchy of available methodologies for sample preparation of complex environmental and biological samples.

\section{BACKGROUND INFORMATION}

\subsection{Sample Preparation in Analytical Chemistry}

Analytical chemistry is the branch of the physical sciences that provides information about an item at the molecular or atomic level. In analytical chemistry, the process of analyzing a sample is generally composed of three broad but distinct parts: sample preparation first, followed by instrumental analysis and lastly data analysis and interpretation. Sample preparation is essentially any treatment done to a sample before instrumental analysis. The second part of the analytical process, instrumental analysis, consists of gathering 
information about a sample with a machine that provides information on the identity of what is contained in the sample and, in some cases, also how much of it there is. In modern analytical chemistry, two commonly used types of instruments are gas chromatographs (GC) and liquid chromatographs (LC), both of which can be coupled to mass spectrometry (MS). Absorbance-based detection such as ultra-violet (UV) are also prominent and important options. The data interpretation is done after sample preparation and instrumental analysis and leads to conclusions on the results of the analysis, whether the purpose is innovation in research or discovery in routine analysis such as the work done in forensic laboratories.

Sample preparation is an umbrella term that includes a vast amount of possibilities that encompass a plethora of different approaches, shapes, requirements and specific goals and expectations. While this section of the analytical process is essential for most samples, it does not always receive as much attention as its significance warrants. As a consequence of the vast majority of samples not being suitable direct introduction into analytical instruments such as high performance liquid chromatography with ultra-violet (HPLC-UV) or tandem mass spectrometry detection (HPLC-MS/MS), [15] sample preparation is necessary and something that is necessary requires foremost consideration. Only if prioritized can sample preparation be turned from something that is viewed by some as bothersome and time-consuming to a great attribute in the analytical process. It will be shown here that sample preparation 
not only can, but should be the essence of the analytical method itself along with optimal instrumental analysis and data interpretation parts of it.

There are many considerations when selecting the best possible sample preparation option for an analytical chemistry method. Some of the goals of sample preparation are necessary, such as making the sample able to be analyzed in the desired instrument, or in some cases just the one available. Other attributes of sample preparation technologies may not be necessary but certainly desirable because these may greatly enhance the effectiveness of the entire analytical procedure. A great example of this is obtaining the best possible preconcentration factor (PCF) (see Equation 1), which compares the concentration of the analytes at the end of the sample preparation procedure to what it was in the original sample.

$P C F=\frac{\text { Conc after sample prep }}{\text { Conc before sample prep (in original sample })} \quad$ Equation 1

Achieving a certain value of PCF is necessary depending on the initial concentration of the compounds of interest in the sample and the sensitivity of the instrument that will be used. Instrumental sensitivity is a measure of the lowest amount of a certain compound that a specific instrument can detect. Let us consider a situation where the instrumental sensitivity for benzene is $50 \mathrm{ppb}$. If, for example, the concentration in the original sample is $100 \mathrm{ppb}$, and the preconcentration factor is 1 , then the solution that is injected in the instrument will be $100 \mathrm{ppb}$. However, if the concentration of the sample had been lower than the 
instrumental sensitivity, say $25 \mathrm{ppb}$, then the preconcentration factor would have to be at least $2(25 \mathrm{ppb} \times 2=50 \mathrm{ppb}$ which is the lower limit of the instrumental sensitivity) for the analytical method to be effective. This is an example of PCF being essential in the sample preparation part of the analysis. Overall, the following are considered important attributes of an ideal sample preparation technique $[15,16]$ : relative simplicity because the simpler the technique the less likelihood there is for errors to be made and it makes it easier to train new scientists to use it; high efficiency and throughput, given by short extraction time and overall time consumption, achieved by integration of sampling, extraction, preconcentration, and sample introduction; not physically demanding so that it can be performed by most people independently of overall physical condition and several samples can be analyzed simultaneously; ability to extract without pretreatment of sample matrix which helps increase sensitivity and improves the accuracy of quantitation while reducing cost and time consumption; high analyte preconcentration (if the main focus is sensitivity instead of quantitation); low cost of production (makes it accessible to more people worldwide); ruggedness because physical, chemical, and physical stability increase the situations where the method is useful and can make devices reusable. Other desirable features are relative ease of automation for essential for large-scale operations, portability when field sampling is necessary, and adherence to Green Analytical Chemistry (GAC) attributes nonexistent or minimal need for organic solvents by use of thermal desorption [17]. 


\subsection{Liquid-Liquid Extraction}

Liquid-Liquid Extraction (LLE) is practically a well-established antique sample preparation technique that is still used in modern analytical chemistry. The technique, which is also known as solvent extraction, is the concept of taking advantage of highly preferential partitioning of a certain compound from one liquid to another [18] and can be useful in many different applications and kinds of laboratories [19]. The two liquids must be immiscible, as much as possible, to avoid making a solution where the sample is simply diluted. Since the two liquids are immiscible, two distinct layers are created. Kinetic energy is applied to the container with these two immiscible liquids, usually in the form of shaking, in order to achieve as much exposure as possible between the two layers. Ultimately, since the compound of interest has much more affinity to one phase than the other, this repeated exposure and refreshment of the border of one phase with the other causes that compound to be transferred to the preferred phase.

As it pertains to real-world applications, one of the phases is typically aqueous, with the other being an organic solvent. There are many applications for analysis of aqueous solutions: environmental (fresh or saline water bodies); biological (blood, urine and milk); food (juices).

When an aqueous solution contains a compound of interest it is then considered the sample matrix. The sample is placed in a separatory funnel (see Fig. 1) along 
with the selected organic solvent. The densest layer of liquid will be the bottom layer.

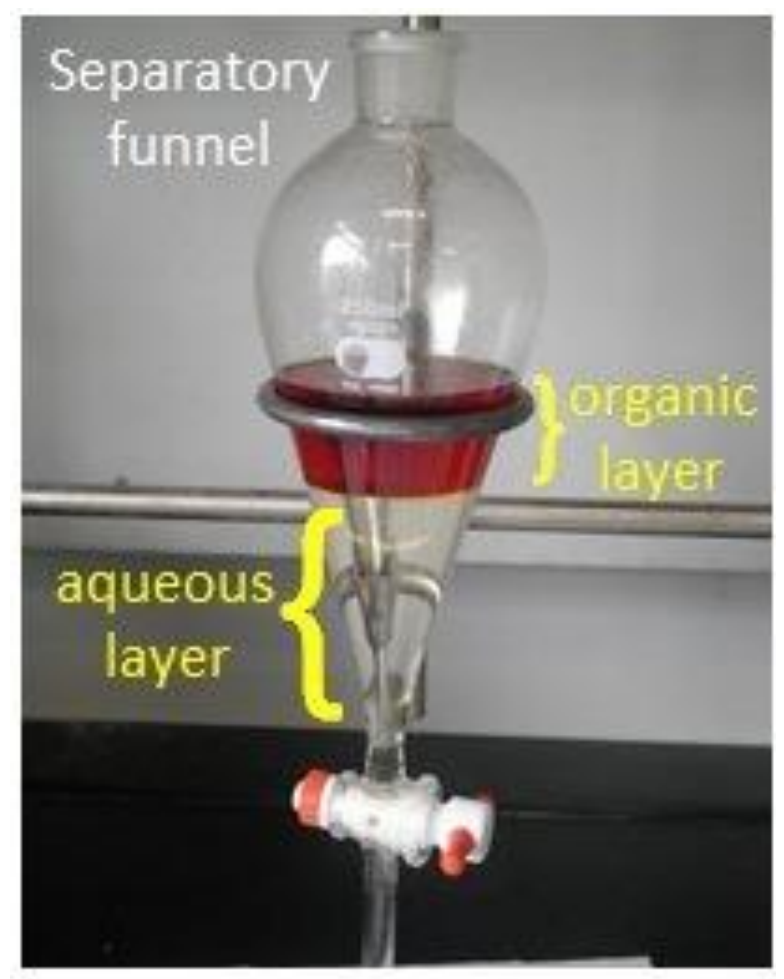

Figure 1: Separatory funnel showing two distinct layers of immiscible liquid, one aqueous and one organic.

The organic solvent is selected such that the analyte has greater affinity to it than to the aqueous matrix that it is already in. This difference in affinity is governed by the partition coefficient $\left(K_{D}\right)$ which depends on the physicochemical properties of the aqueous matrix and the organic solvent. After shaking of the funnel to force the two layers to come in contact with each other, the analytes are present in both layers. However, since the partition is preferential to the organic layer, most of the analytes will have left the aqueous matrix. After shaking the 
separatory funnel it should be vented, following proper procedure for safety reasons (see Fig. 2), because of dangerous formation of vapors.

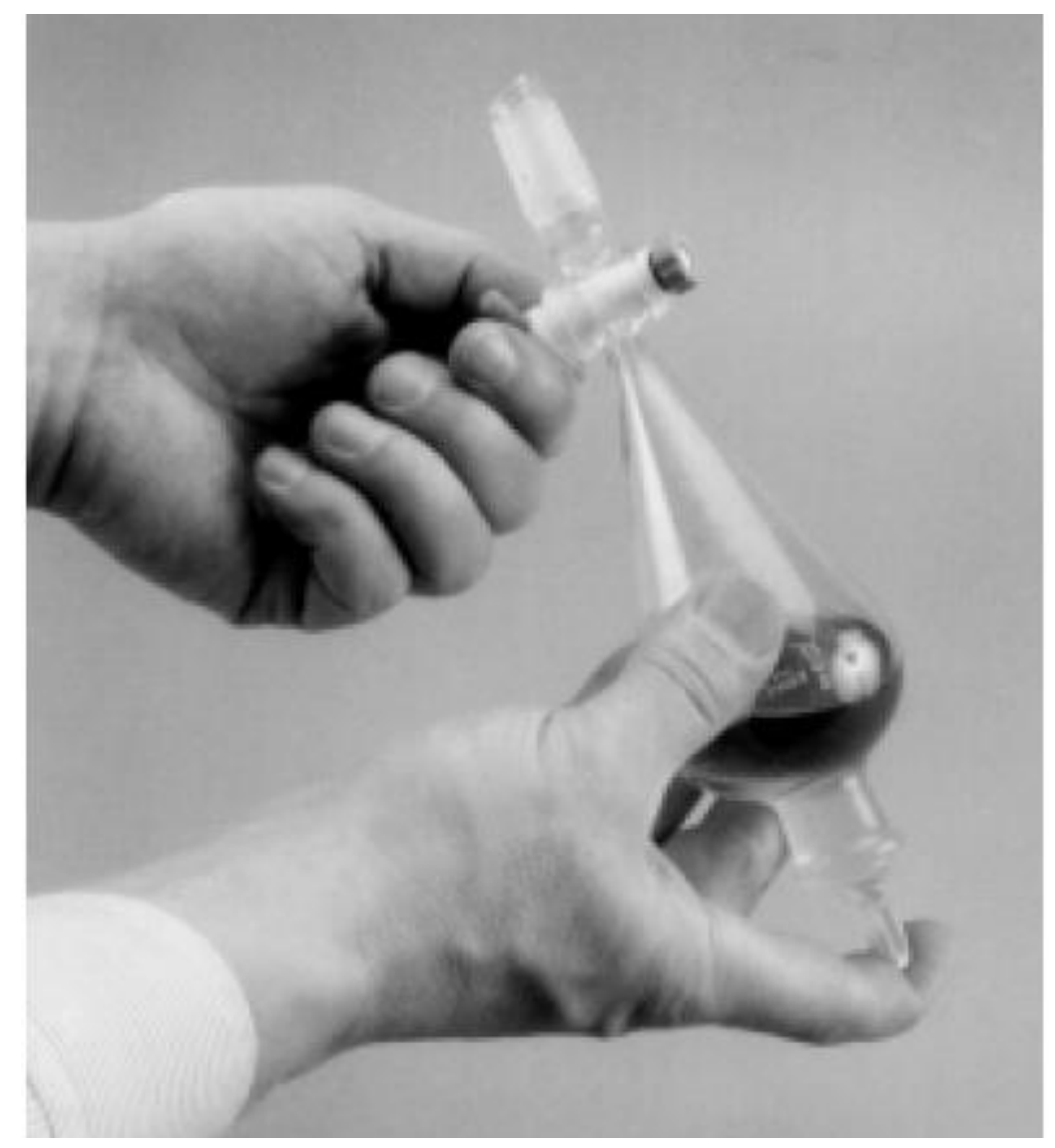

Figure 2: Demonstration of proper procedure for venting a separatory funnel. The effectiveness of the results of the procedure is generally measured by the extraction efficiency: the percentage of the analyte having moved from the aqueous to the organic phase [19]. The extraction efficiency is affected by several factors including acid-base and complexation reactions and is given by Equation 2, where $K_{D}$ is the partition coefficient, $S_{\text {org }}$ is the concentration of solute in the organic solvent and $S_{a q}$ is the concentration of solute in the aqueous matrix [19]. 
$K D=\frac{\text { sorg }}{\text { Saq }}$

If there are no acid-base considerations, the solute's partitioning is the only factor determining the extraction efficiency (Fig. 3).

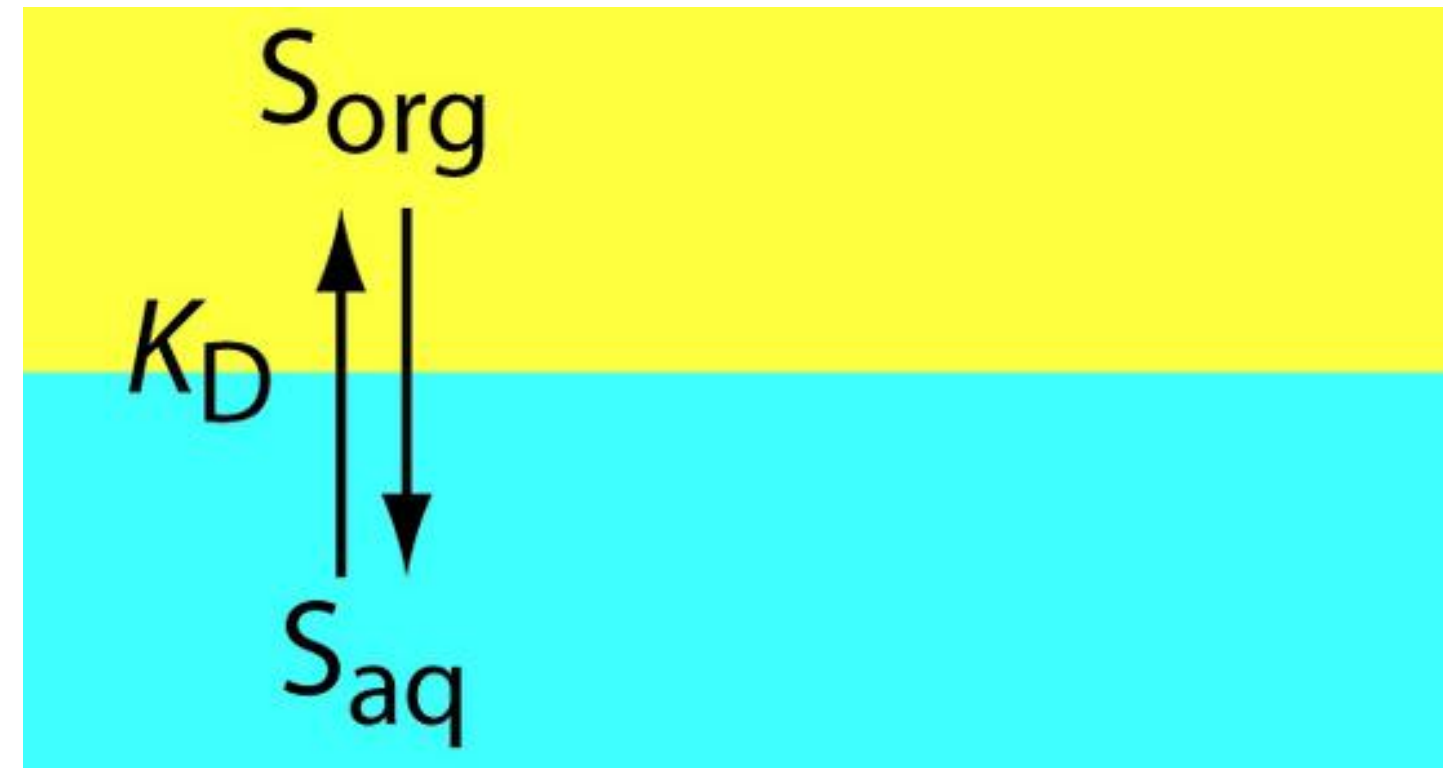

Figure 3: LLE scheme where the partitioning of analytes depends solely on $K_{D}$ [19].

Alternatively, if there are acid-base reactions that need to be accounted for, the extraction efficiency is determined by more than just the partition coefficient (Fig.

4): In this case the partition coefficient also depends on the acid dissociation constant Ka because the relative amount of the protonated form of the acid HA depends on it. 


\section{organic phase}

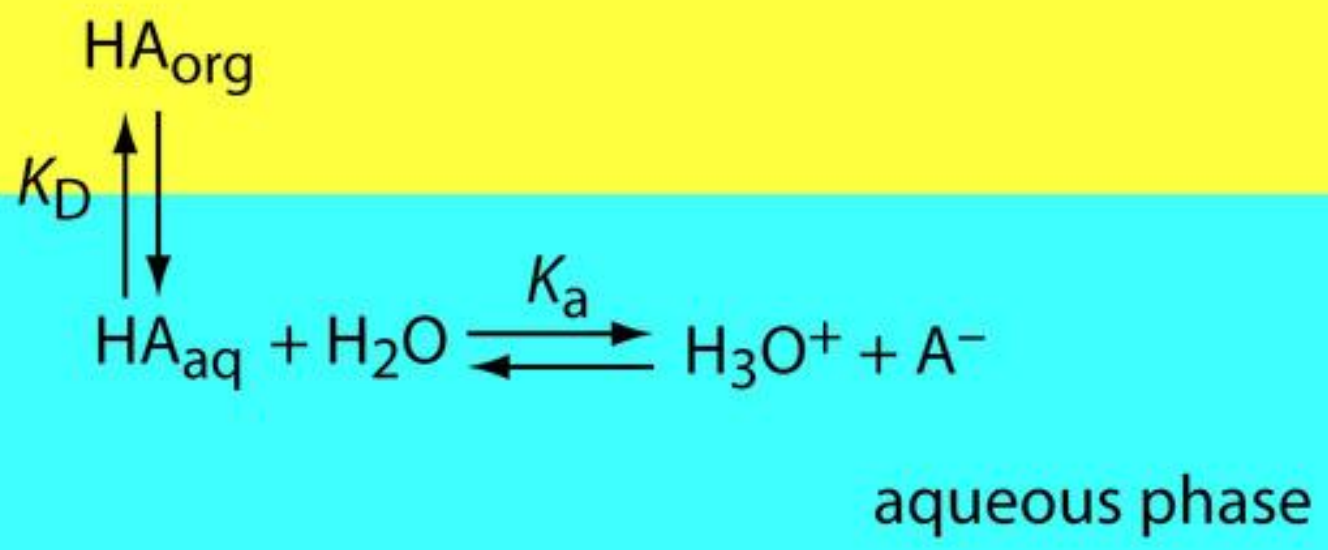

Figure 4: LLE scheme for a weak acid, where the partitioning of analytes depends on $K_{D}$ but also the relative abundance of the protonated acid HA, given by the acid dissociation constant $\mathrm{Ka} \mathrm{[19].}$

When using LLE, extracting several times can help maximize the amount that is extracted, but does not necessarily increase the resulting concentration, because each extraction obtains less solute than the previous process. Generally, after the third extraction the amount of solute gained is so small that it is not worth the time and effort to perform a fourth extraction (Fig. 5). This lack of efficiency is quite unfortunate because LLE is laborious, time-consuming, wasteful, and harms the environment and for those reasons it is less than ideal to have to perform a series of extractions to extract a large percentage of analytes. 


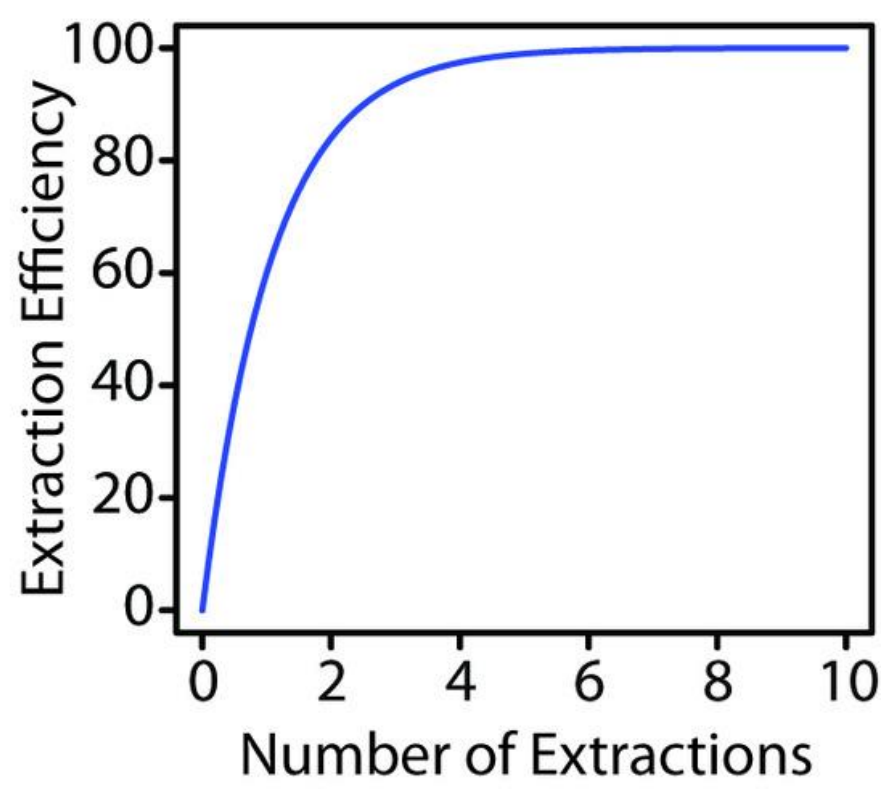

Figure 5: Representative plot of extraction efficiency vs number of extractions in LLE [19].

If there is an acid-base equilibrium that needs to be accounted for, the higher the relative concentration of the neutral species the better the extraction efficiency. If the analyte is a weak acid $\mathrm{HA}$, the $\mathrm{pH}$ being at least two units lower than the corresponding pKa will result in the best results (Fig. 6). If it is a weak base, the best possible results are achieved by increasing the $\mathrm{pH}$ to be at least two units above the pKb. 


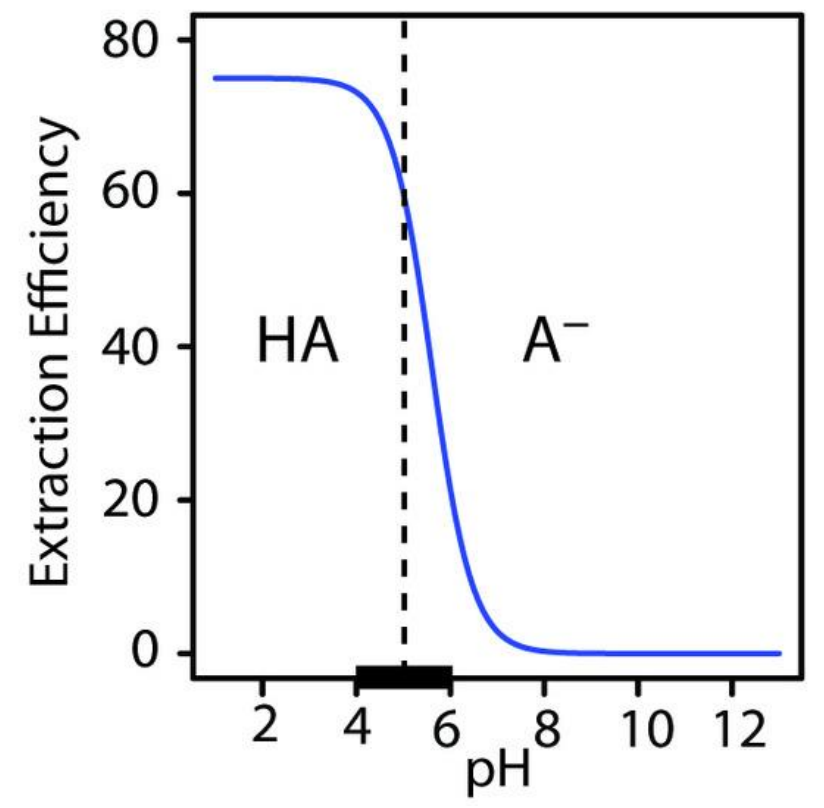

Figure 6: Plot of extraction efficiency vs $\mathrm{pH}$ of solution for a weak acid HA [19].

While LLE is still used in some laboratories and was once an incredible breakthrough in analytical chemistry, it has grave shortcomings:

Not acceptable under green analytical chemistry guidelines [17]

- This is the most important drawback of LLE: it requires large volumes of organic solvents, which is not at all compliant with GAC mandates. The GAC mandates are paramount in the modern world because of an ever-growing desire to protect the environment.

Evaporation and reconstitution

- In LLE, it is almost always necessary to perform analyte evaporation and reconstitution after extraction because the volume of the large amount of organic solvent. This process is done to 
procure a better PCF but is not ideal because it makes the analysis time hours longer and adds error to the quantitation.

$>$ Emulsion formation [20]

- Emulsions are small droplets of one of the liquids suspended in the other that lead to poor separation if not destroyed because those droplets will retain analyte that will not be analyzed. Destroying emulsions is very inconvenient because it usually requires lots of time or using additives.

$>$ Overly laborious [20]

- The amount of energy required to make two immiscible liquids interact is tiring and excludes some scientists who are otherwise qualified to perform the procedure.

\subsubsection{Liquid-Liquid Microextraction}

Growing concerns for environmental protection and the desire to pursue options that follow GAC principles led many researchers to focus on developing microextraction (ME) techniques, starting in the 1980s, which makes the approach relatively new $[21,22]$. One example of a ME sample preparation technique is a derivative of LLE: Liquid-Liquid Microextraction (LLME), which was introduced in the 1990s [23]. The difference between LLME and LLE is that LLME uses a much smaller amount of organic solvent (a few $\mu \mathrm{L}$ ) than LLE, which typically requires an amount measured in $\mathrm{mL}$ [21]. Mechanistically speaking, the specific objective of LLME is quite different from LLE because it focuses on creating preconcentration by using a very small volume of organic solvent 
instead of extracting as much of the analytes as possible [21,24]. While LLME addresses one of the major issues of LLE by being a GAC technique, it is still quite flawed because it retains a small absolute amount of analytes.

\subsubsection{Dispersive Liquid-Liquid Microextraction}

Approximately a decade after LLME was introduced as a derivative of LLE, Dispersive Liquid-Liquid Microextraction (DLLME), developed as a derivative of LLME. First introduced by Rezaee et al. in 2006 [25], DLLME consists of mixing the aqueous matrix with an immiscible organic solvent, just like LLME, but also uses a dispersive solvent (miscible in both the aqueous sample and the extractant); this results in microdroplets forming and a high contact surface area between the extractant and the analytes [25]. A centrifugation step is necessary to separate the two components [25]. The extraction efficiency is improved compared to LLME because of the high contact surface area and the amount of extractant used is much lower than that of the aqueous sample, leading to a high PCF [25]. Several applications of applications have been found and published for DLLME as a sample preparation technique $[26,27]$, but while it provides some improvement over LLME and maintains the GAC principles, it does not completely solve the problems of LLME and is still an overly laborious process that makes quantitation very challenging.

\subsection{Solid Phase Extraction}

Solid Phase Extraction (SPE) became the most prevalent and commonly used sample preparation technique late in the 1990 s, replacing LLE, to the extent that 
more than 50 companies were producing SPE products by the beginning of the $21^{\text {st }}$ century $[28,29]$. The first SPE disposable cartridges were made available in 1978 , followed by syringe cartridges in 1979 , so this major change in preference for sample preparation did not happen as a consequence of a sudden availability of better commercial products or ease of use, but rather because SPE is greener than LLE $[29,30]$.

The SPE apparatus, most commonly in the form of disposable cartridges (Fig. 7) packed with silica particles $(50-60 \mu \mathrm{m})$ that have a stationary phase (also referred to as the sorbent) on them, is arguably nothing more than a miniature LC column [31]. Just like LC columns, the SPE stationary phase retains analytes by adsorption, absorption, or both, depending on the analyte and the identity of the stationary phase. The effectiveness of the retention of SPE stationary phase is given by the same a partitioning coefficient, as seen previously with LLE (Equation 1). There are many different stationary phases available for SPE, ranging from all-purpose sorbents like $\mathrm{C}-18$ to targeting specific polarities and functionalities and even compound specific options by using molecularly imprinted polymers [31-33]. 


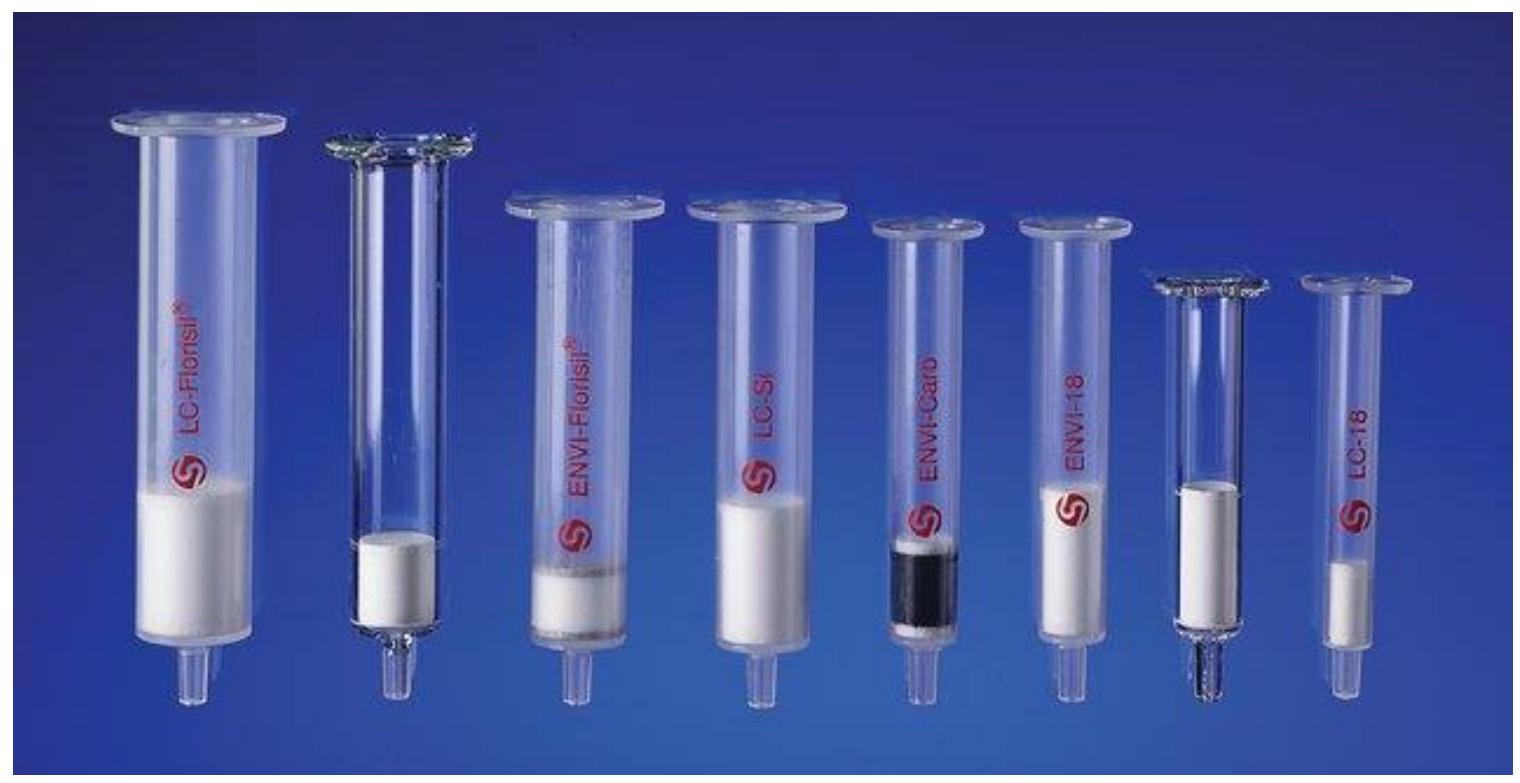

Figure 7: Image of various commercially available SPE cartridges including plastic and glass construction [34].

An alternate design of SPE employs disks (Fig. 8) instead of columns, which improve on the negative characteristics of the SPE cartridges by reducing the resistance to the flow of the sample and as a result make the process faster and applicable to matrices that are a bit denser as compared to SPE cartridges $[35,36]$. SPE disks also have the added advantages over SPE cartridges of exhibiting more reproducible synthesis, requiring less sample size because of smaller elution volume, and minimizing non-specific matrix adsorption [31]. The disks are composed of much smaller particles, suspended in a polytetrafluoroethylene (PTFE) membrane, than those of the cartridges. Using smaller particles is made possible because since the thickness is reduced exponentially, it is still easier to pass a sample through the disks than the cartridges [31]. The size of these particles is only $8-10 \mu \mathrm{m}$, while the disks are only $0.5 \mathrm{~mm}$ thick with a wide range of possible diameters [31]. 


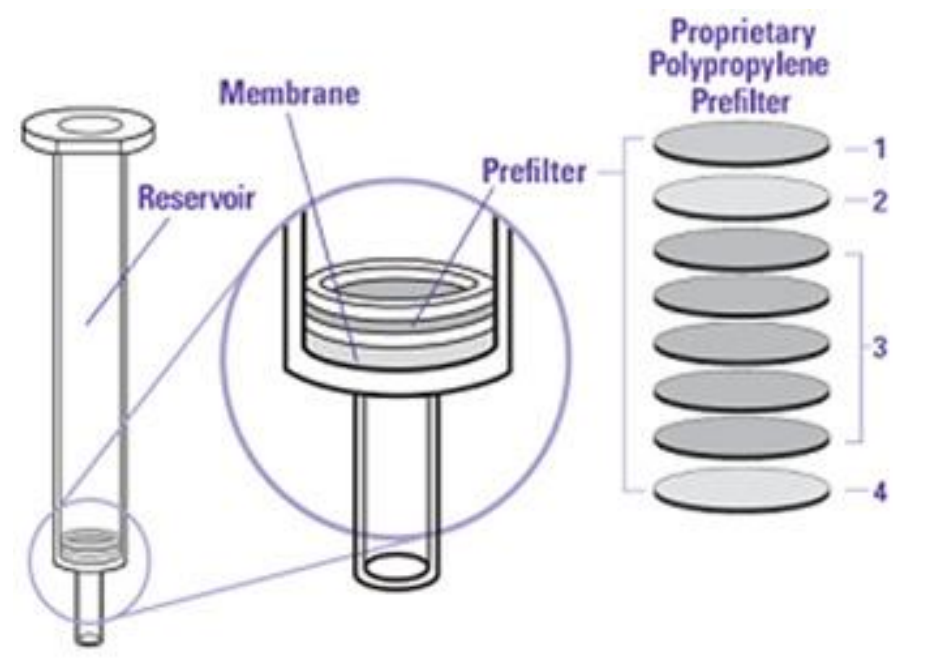

Figure 8: Schematic of composition of SPE device employing disk technology instead of a packed cartridge [37].

SPE procedure requires several steps that make the SPE procedure a troublesome and time-consuming sample preparation technique [31,38-40]: first, conditioning of the sorbent to diminish the amount of sorbent impurities that can affect eluted solution and to lower unit to unit variability, thereby improving repeatability. Even water can pose problems for SPE flow control, as the high surface tension of water combined with the microporosity of the particle-loaded membranes results in slow and uneven flow and low analyte recovery if the membranes are not first conditioned properly with an organic solvent [24]; second, acclimation of the stationary phase to the solvent of the sample by passing it through before the actual sample; third, passing the sample through the cartridge so that the sorbent can retain the analytes; fourth, rinsing of the sorbent to displace unwanted compounds of the matrix by using a solvent with very weak affinity to the analytes so that as few of them as possible are lost; fifth, trying to prevent carry-over water to interact with the eluting solvent, therefore 
reducing eluting efficiency and increasing volume of solution after elution; sixth, elution from the stationary phase with an organic solvent that has as much affinity to the analytes as possible; seventh, it is most common that for trace or ultra-trace analysis, solvent evaporation and reconstitution is carried out to reduce the volume of the resulting solution with the analytes and therefore increase the PCF and sensitivity of the method.

Since there is a great variety of stationary phase options for SPE, it can be difficult to select the best sorbent. There are guides that have been compiled based on scientific theory and experimental results, although their accuracy is not always satisfactory [31]. Figure 9 present one such guide for selecting SPE sorbents based on analyte chemical properties and the general category of the sample matrix [31]. 


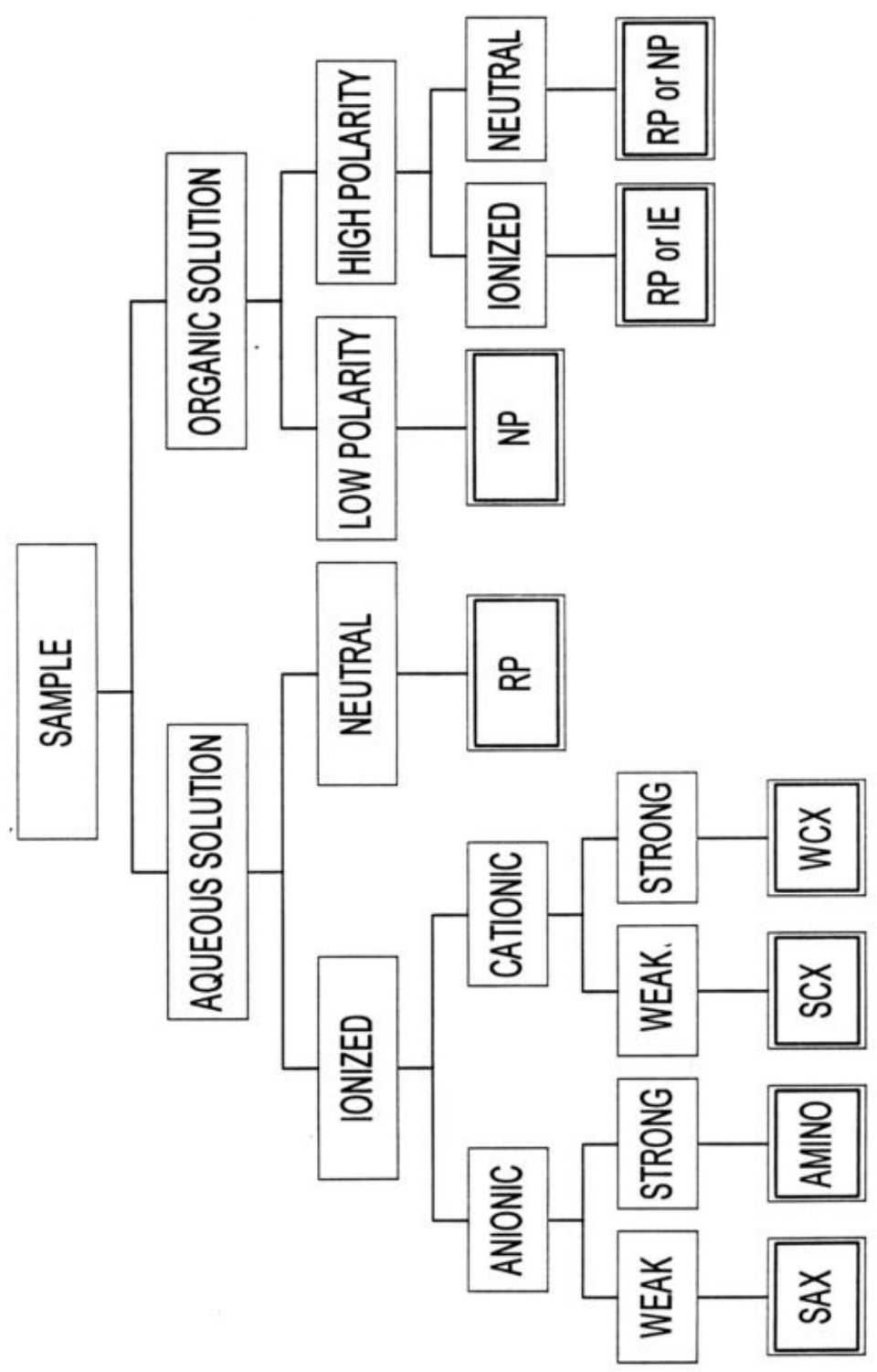

Figure 9: Guide for stationary phase selection for organic compounds from aqueous and organic matrices. SAX, strong anion exchanger; SCX, strong cation exchanger; WCX, weak cation exchanger; RP, reversed-phase sampling conditions; NP, normal-phase sampling conditions; IE, ion-exchange sampling conditions.

Solid Phase Extraction is commercially available in off-line as well as on-line formats [29], with the on-line version being faster and less laborious but also abundantly more costly and only sensible for laboratories that require high through-put for many samples. Off-line mode requires the analyst to manually 
pass the sample, as well as the subsequent solvents, through the cartridge, handling only one extraction at a time, before performing analyte evaporation and reconstitution and instrumental analysis. On-line mode, on the other hand, does not involve direct handling after loading the sample because it is couple to liquid chromatography and so the elution goes directly into the instrument $[29,30,41-$ 44]. The advantages of on-line SPE extend beyond being less laborious and more time efficient than traditional SPE: there is little to no risk of sample contamination since handling the process manually is not required [29]. On-line mode excludes analyte evaporation and reconstitution, which is possible without sacrificing sensitivity because all the elution solvent is introduced to the HPLC, whereas in off-line mode only a small aliquot of it is injected in the instrument unless evaporation and reconstitution is done. This represents a shift in focus, somewhat similar to the shift in focus from LLE to LLME, which is the preference of reducing the volume of solvent while sacrificing some of the amount that is actually extracted. It is worth considering that injecting all the elution solvent may also create a serious chromatographic problem because the efficiency of the peaks would certainly be diminished and as such the resolution as well.

There is no doubt that SPE has been a serviceable sample preparation technology for decades, but it is also universally accepted that it presents serious challenges and has some very important downsides. The first problem encountered with SPE is that, as described previously, the procedure is regularly hours long, arduous and generally inefficient because of the many steps required $[31,39,40]$. A second big disadvantage of SPE is that most of the material that 
the sample is passed through, whether it is a packed cartridge or a disk, is not stationary phase. This lack of functional stationary phase results in most of the material not being available for retention of analyte. Solid Phase Extraction cartridges and disks are dense and because the sample must be passed through, the sample cannot be dense or have too many macroparticles, which is often the case with real-world samples. For biological samples, such as milk or blood, for example, protein precipitation and defatting are necessary before starting the SPE procedure, but this has monumental downsides: makes the process even longer, more costly and inefficient; sacrifices a great deal of sensitivity because fat and proteins essentially act as separate stationary phases that retain analytes; makes quantitation inaccurate because it is not known how much is lost in the treatment of the sample before the SPE procedure. In the elution step it is imperative that the breakthrough volume is estimated accurately (or determined in previous trials) and that it is taken into account (see Fig. 10) [31]. Breakthrough volume is the volume of the elution solvent at which the analytes actually start to leave the SPE cartridge or disk. Additionally, it is common for SPE methods to require solvent evaporation and analyte reconstitution and this, as noted previously, creates its own set of inconveniences and further extends an already notoriously long procedure. 


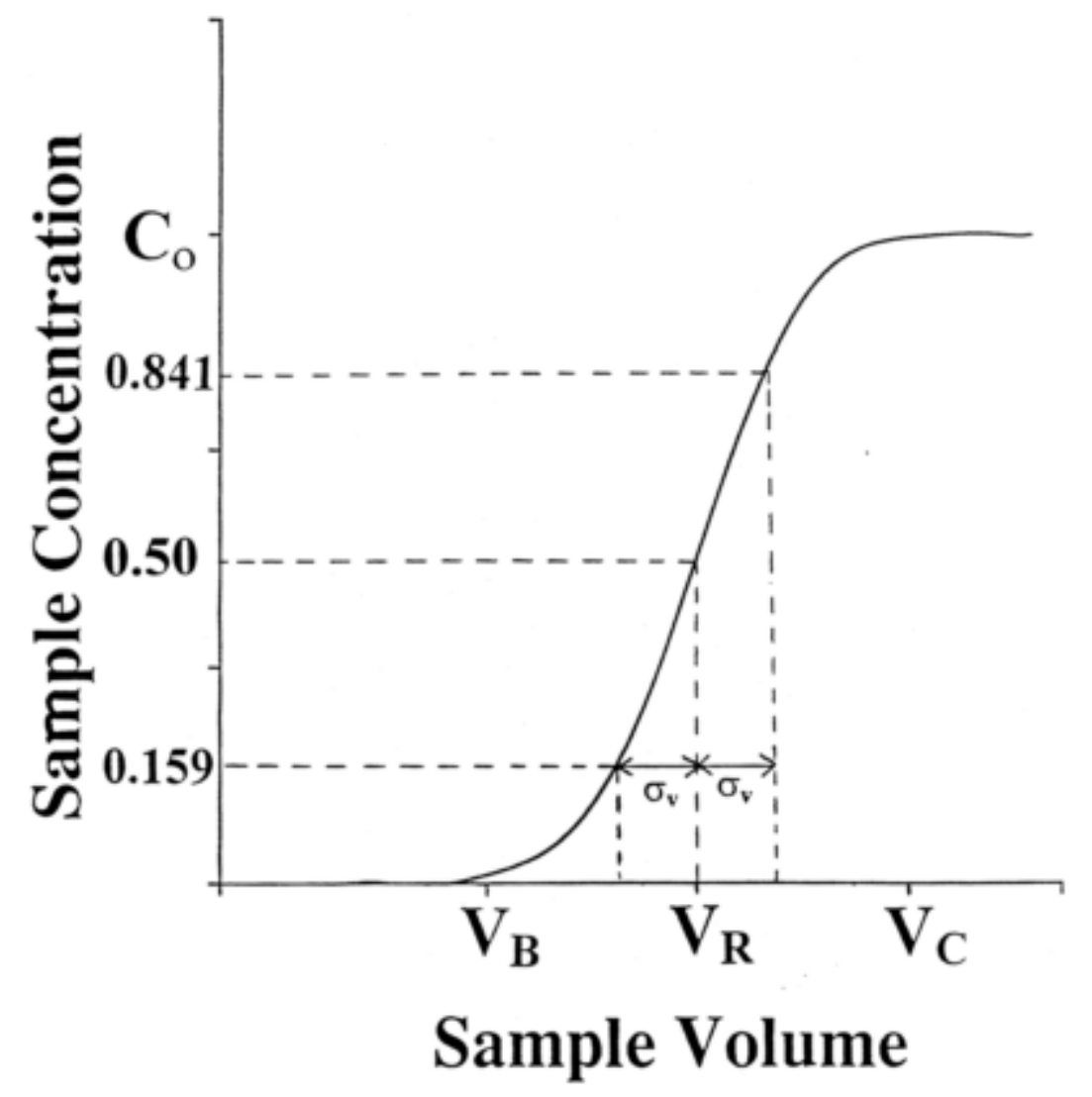

Figure 10: Graphical representation of curve used to determine breakthrough volume. $V_{B}$ stands for breakthrough volume; $V_{R}$ for the chromatographic elution volume; $V_{C}$ for the sample volume corresponding to the isolation of the maximum amount of analytes; $\mathrm{C}_{0}$ for the concentration of analyte in the sample; $\sigma \vee$ for the standard deviation of the derivative curve for the plot.

\subsection{Solid Phase Microextraction}

Microextraction techniques have gotten garnered great traction in the last two decades or so for sample preparation technologies because they provide advantages in addition to simply adhering to GAC principles. Of the aforementioned ideal traits of sample preparation technologies, ME techniques are inherently better at the following compared to traditional options such as LLE and SPE [15]: simplicity that reduces training time of new scientists and likelihood of experimental errors; high efficiency and throughput, especially due 
to integration of sampling, extraction, preconcentration, and sample introduction; high analyte preconcentration by using little or no organic solvent for elution of analytes; relative ease of automation by dramatically increases efficiency for large-scale operations; acceptable portability with effective field sampling; adherence to GAC principles [17].

The benchmark ME technique is undoubtedly Solid Phase Microextraction (SPME), which was a real breakthrough in sample preparation technologies. A creation of Dr. Janusz Pawliszyn at the University of Waterloo (Waterloo, Ontario, CAN), SPME was first introduced to the scientific community in 1990 [45]. Designed to diminish or eliminate some of the problems of SPE, the physical composition of SPME is quite simple: a fused silica fiber coated with a stationary phase held inside a syringe-like exposure device. The original schematic of the construction of this device can be seen in Fig. 11 [45], which also shows a diagram of a modern commercially available SPME fiber [46].

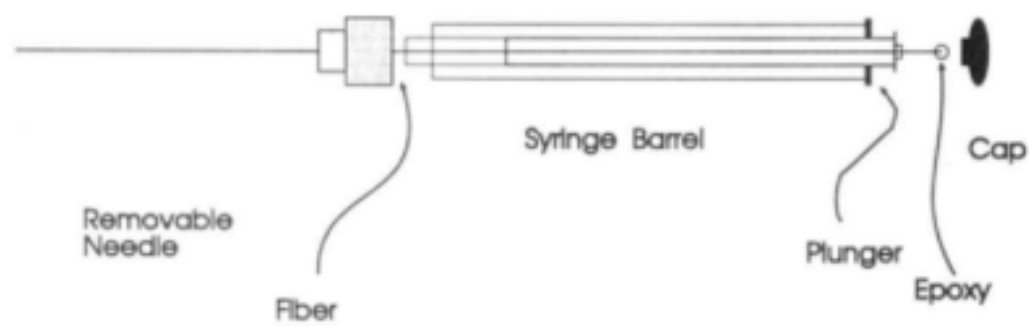




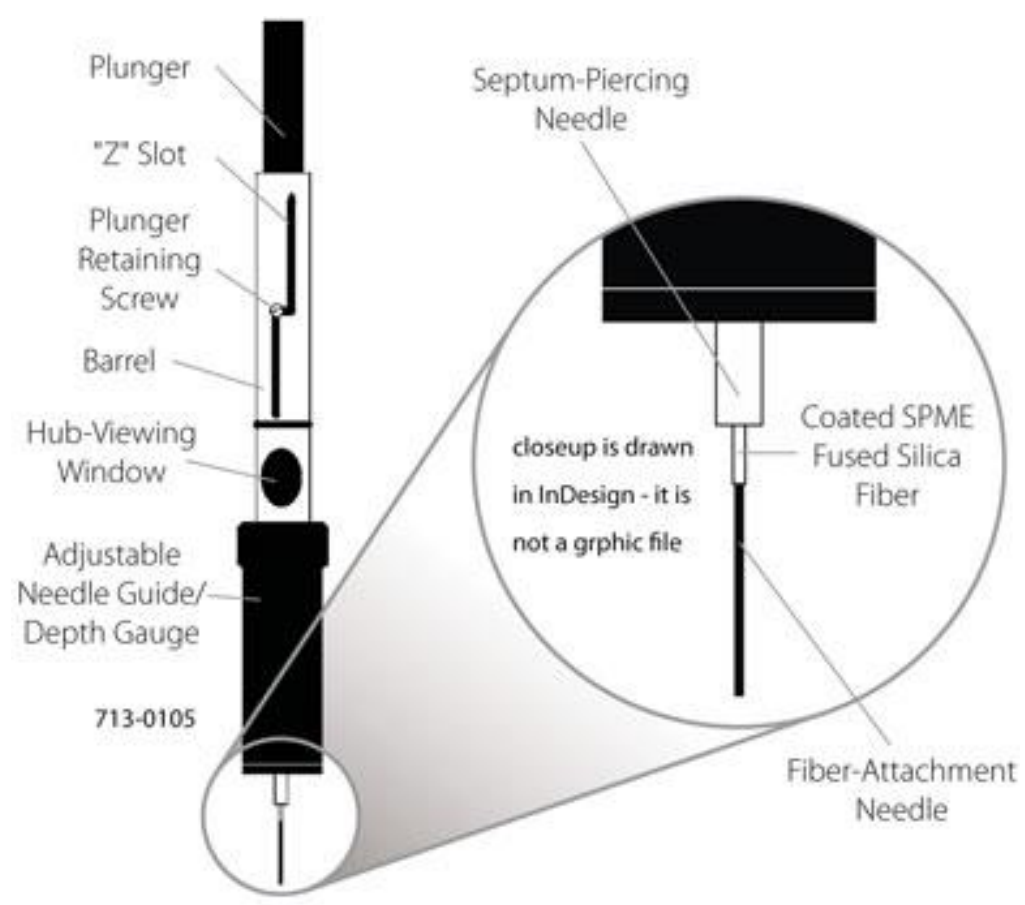

Figure 11: Top: Original schematic of SPME apparatus construction. Bottom: Schematic of a commercially available SPME fiber with exposure apparatus.

Solid phase microextraction brought unprecedented simplicity and ease of use to sample preparation and improves upon SPE by not violating GAC principles and making thermal desorption for GC analysis not only more practical but truly seamless [45,47]. The SPME device can be used like a syringe to push out the coated fused silica fiber into an aqueous matrix or headspace of a sample. Once exposed to the analytes, the stationary phase retains a certain amount of the analytes, the fiber is retracted into the syringe-like device and the analyst has two options: back extract with a small amount of organic solvent or thermally desorb the analyst directly into a GC. Back extraction is technically valid for both GC and LC analysis, but only chosen for LC because thermal desorption is more practical and all the analytes are introduced into the instrument for analysis. 
The retention of the analytes occurs by equilibrium adsorption, which means that the extraction mechanism depends on a partition between the sample and the stationary phase $[45,47]$, not unlike the mechanism that governs LLE and its derivative techniques LLME and DLLME. Equation 3 is a representation of this equilibrium extraction mechanism, where $\mathrm{K}$ is the distribution constant, $\mathrm{C}_{\mathrm{s}}$ is the concentration of analyte in the stationary phase and $\mathrm{C}_{\mathrm{aq}}$ is the concentration of the same analyte in the aqueous matrix $[45,47]$.

$K=\frac{C s}{C a q}$

Equation 3

Solid phase microextraction was immediately found to exhibit a linear relationship between the amount adsorbed on the stationary phase and the concentration in the sample, but only at relatively low concentrations [45,47]. From Equation 3, it follows that the partition ratio, $k^{\prime}$, is given by Equation 4 , where $V_{s}$ and $V_{a q}$ are the stationary and aqueous phase volumes, respectively, and $n_{s}$ and $n_{a q}$ are the number of moles of analyte in each phase, respectively [45].

$k^{\prime}=\frac{C s V s}{C a q V a q}=\frac{n s}{n a q}=K \frac{V s}{V a q}$ Equation 4

Equation 4 can be rearranged to produce Equation 5 [45]:

$n s=K * V s \frac{n a q}{V a q}$

Equation 5

Substituting $C_{a q}{ }^{*} V_{a q}$ into Equation 5 for $n_{a q}$ then yields Equation 6 where $A=K^{*} V_{s}$ [45]: 
$n s=K * V s * C a q=A * C a q$

Equation 6

Equation 6 clearly shows a linear equation in the form $y=m x$, even if the high end of this linearity is relatively low.

The significance of SPME as a breakthrough technique with a revolutionary design is undeniable and it is still used extensively, especially for head space extractions followed by GC instrumental analysis. However, SPME does have flaws that needed to be addressed. In terms of physical design, the structure of traditional SPME apparatus allows for the stationary phase on the fiber to be unprotected while sampling and subject to scraping, breakage, and bending [16]. The stationary phase is only placed on a short segment of the fiber, providing an extremely low primary contact surface area (PCSA) of active sites for retention of analytes and therefore a low sample loading capacity [16]. The low PCSA is one of the major drawbacks of SPME because it limits the sensitivity of the technique. Increasing the thickness of stationary phases has been proposed as possible solutions to a low PCSA, but the increased thickness makes it more difficult to immobilize them on the fiber [48]. Increasing the thickness of the coating also presents a drawback in that the extraction process is diffusion controlled and equilibration time is proportional to the thickness of the coating, so the amount of time necessary for extraction increases and so does the efficiency [16] (see Fig. 12) [47]. The reason that it is difficult to immobilize thick coatings on fibers is that this is done by physical deposition; the coating does not bond to the fiber [16]. The lack of bonding undermines the ruggedness of SPME in general and leads 
to poor solvent and chemical stability [16]. Solvent stability is a measure of resistance to damage associated with prolonged exposure to organic solvents. Chemical stability refers to resistance to damage that results from exposure to highly acidic or highly basic solutions.

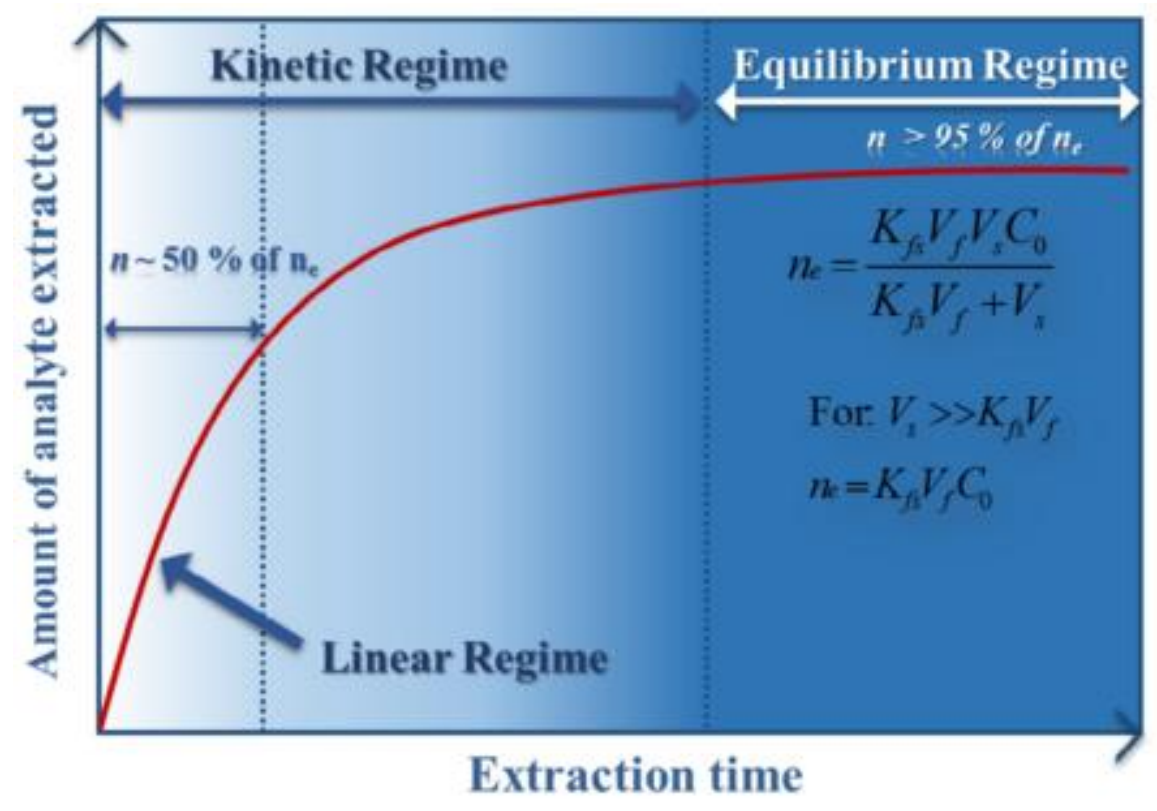

Figure 12: Plot of relationship between extracted amount and extraction time for SPME, including the linear, kinetic, and equilibrium regimes.

Solid phase microextraction is extremely simple and practical for headspace analysis of a sample, as explained previously, but for direct immersion analysis SPME is not considered nearly as adequate because the coating is not porous and particles in solution block access of the analytes to active sites on the coating [15]. The lack of direct immersion effectivness is another major drawback of SPME because while headspace analysis is adequate and convenient in some situations, there are cases when it is not as valid an approach as direct immersion because headspace analysis is biased in favor of volatile compounds 
so it does not always provide a fair representation of the composition of a sample. For instance, if an aqueous sample includes a combination of a relatively nonpolar compound, such as phenanthrene (a PAH), and another that is more polar, such as trinitrotoluene (a nitroaromatic explosive), headspace extraction of this sample will be heavily biased towards phenanthrene. Phenanthrene is not nearly as hydrophilic as trinitrotoluene because trinitrotoluene is much more polar and has nitrogen and oxygen atoms that induce hydrogen bonding with water molecules. This difference in hydrophilicity leads to phenanthrene being much more prone to leave the aqueous matrix into the headspace than stay in it. Moreover, if an analyte is hydrophilic enough, the analyte-matrix interactions may be so strong that a very small fraction of the analytes leaves the solution and headspace analysis would be rendered useless.

\subsubsection{Stir-Bar Sorptive Extraction}

Stir-Bar Sorptive Extraction (SBSE), is a term coined by Baltussen et al. in 1999 [49], although the technology was actually introduced two years earlier [50,51]. Stir-bar sorptive extraction is considered an alternative form to or derivative of SPME $[15,49]$. From a structural perspective, SBSE deviates significantly from traditional SPME $[15,49]$, as it consists of a bed of pure PDMS particles placed between a magnetic stir-bar and a glass jacket and has one major advantage over SPME: the sorbent loading capacity is much higher ( $24 \mu \mathrm{L}$ in SBSE vs. 0.5 $\mu \mathrm{L}$ in traditional SPME) $[15,49]$. The mechanism of analyte retention of SBSE is essentially the same as that of SPME, given by the partitioning coefficient, so the added sample loading capacity is as a consequence of the additional stationary 
phase and PCSA [49]. Originally SBSE was designed to use PDMS only as the extraction sorbent but other options have since been made available, with stationary phases such as poly-ethylene glycol (PEG) [15].

Despite the improvements of SBSE over SPME, there are some practical considerations that still favor SPME: if thermal desorption is not performed after extraction, SBSE requires a larger back extraction solvent volume than SPME (which decreases the preconcentration benefit of the sample preparation method and therefore the sensitivity); if thermal desorption is used, an expensive thermal desorption unit is necessary for SBSE and not for SPME [15]. Similarly to SPME, SBSE has also been adapted to headspace analysis [52].

\subsubsection{Thin-Film Microextraction}

After the creation of SPME and a tremendous growth in popularity in a very short period of time, Thin-Film Microextraction (TFME) was introduced by Dr.

Pawliszyn in 2003 [53]. A derivative of SPME, TFME, uses a thin sheet of a stationary phase such as polydimethyl siloxane (PDMS) instead of placing a relatively thick coating of said stationary phase on a fused silica fiber [53]. This new design concept was intended to correct one of the glaring flaws of traditional SPME: the low PCSA and therefore low sample loading capacity [53].

The TFME device is very similar to that of traditional SPME. As can be seen in Fig. 13a, the thin film is attached to a fiber, this time made of stainless steel, and made to interact with the sample as much as possible while being exposed like a flag [53], resulting in maximized interactions with the sample. Fig. 13b shows an 
intermediate step that is not part of the SPME procedure, as the thin film is rolled along the stainless steel fiber. Figure $13 \mathrm{c}$ demonstrates the rolled membrane inserted in a gas chromatography inlet for thermal desorption and subsequent instrumental analysis [53].

a

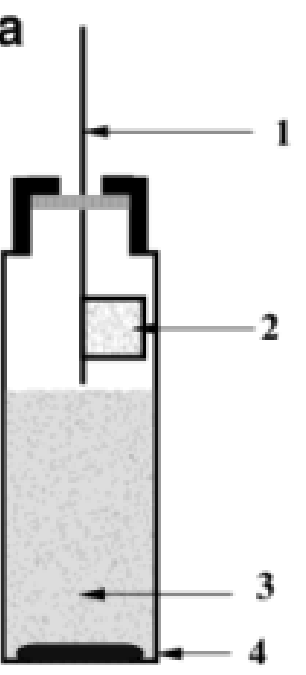

b

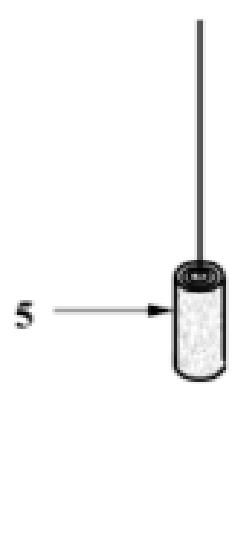

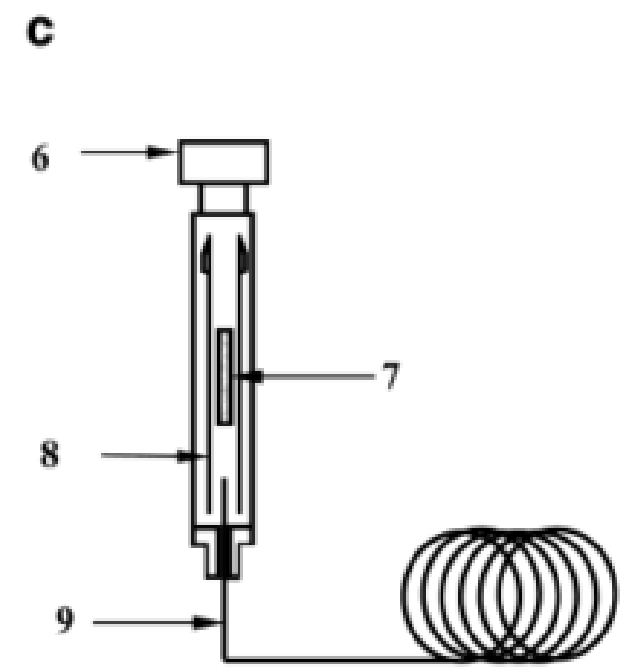

Figure 13: Schematic of the composition of TFME apparatus and steps involved in the corresponding headspace analysis procedure. 1. deactivated stainless steel rod; 2. flat sheet membrane; 3. sample solution; 4. teflon-coated stirring bar; 5. membrane; 6 . injector nut; 7. membrane; 8. glass liner; 9. capillary column.

Traditional SPME, SBSE and TFME all fall in the category of sample preparation techniques that utilize diffusion mediated by stirring. There are also derivatives of SPME that instead of utilizing diffusion mediated by stirring depend on the sample being flowed through the stationary phase. Examples of flow through diffusion sample preparation techniques that come from SPME are In-Tip SPME, Syringe SPME and In-Tube SPME [15]. These six sample preparation options are summarized ion Fig. 14 [15]. In-Tube SPME has also been referred to as Capillary Microextraction (CME) [16,54]. 


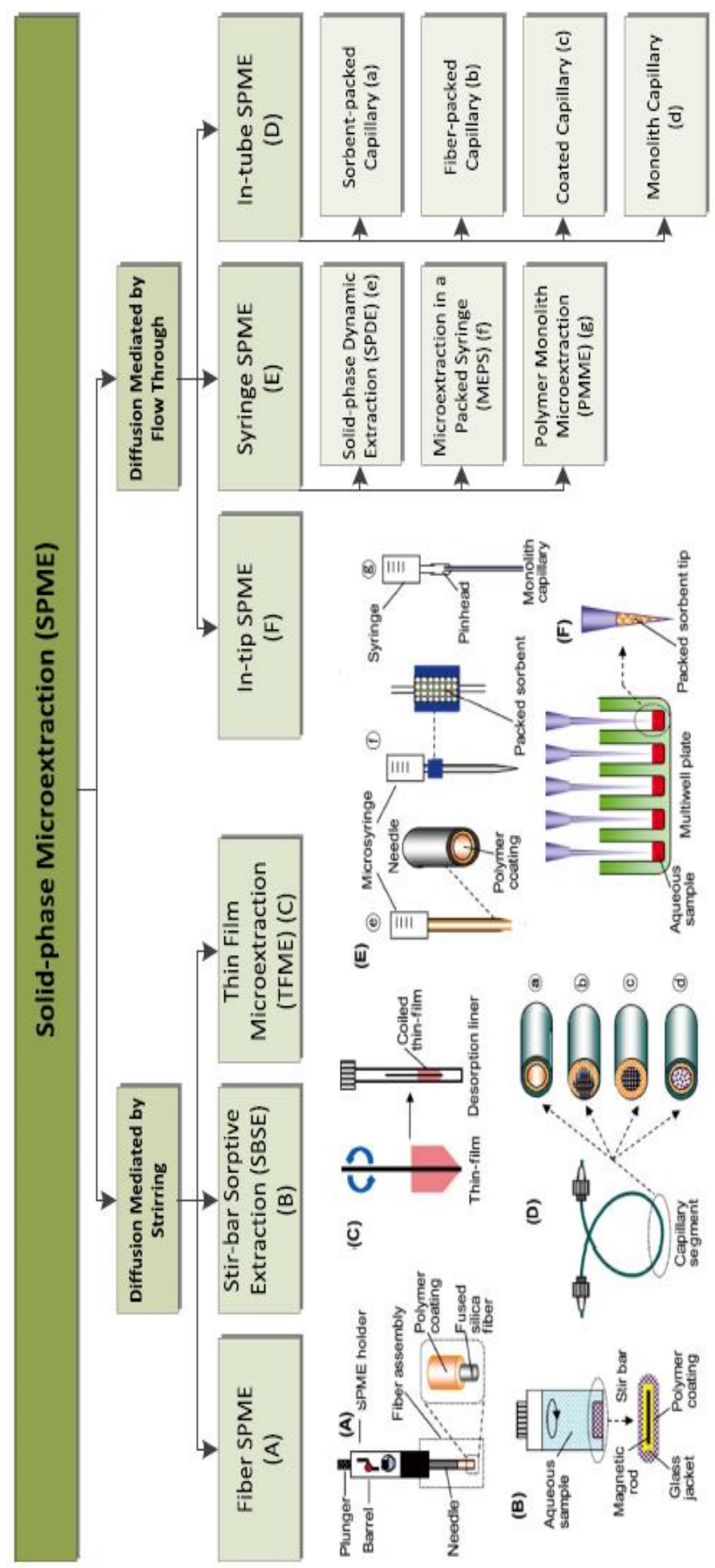

Figure 14: Schematic summarizing traditional SPME, its major derivatives and some distinguishing characteristics [14]. 


\subsection{Fabric Phase Sorptive Extraction}

Because of the numerous, previously outlined and very consequential deficiencies of the leading commercially available sample preparation techniques for trace and ultra-trace direct immersion analysis of environmental and biological samples, it became imperative that another option was developed. Not just another option, but one that could provide exemplary performance without so many consequential compromises. That elusive technique was indeed invented and developed, it is called Fabric Phase Sorptive Extraction (FPSE). A creation of Drs. Abuzar Kabir and Kenneth G. Furton from Florida International University, [14] FPSE and its effectiveness is already starting to become widely accepted via peer-reviewed literature [10,55-66].

\subsubsection{FPSE Theoretical Considerations}

The basic theory explaining the retention of analytes by FPSE sorbents is essentially the same as that of SPME, previously portrayed in Equations 3-6. Simply put: analytes come in contact with the sorbent and are adsorbed or absorbed by the sorbent because the affinity of the analytes to the sorbent is greater than it is to the sample matrix. According to Equation 6, the amount of analytes extracted by the sorbent is directly proportional to its volume and therefore its thickness since this is how volume is commonly increased. However, as sorbents are made thicker but not porous, the increase in PCSA is minimal, thus inhibiting the extraction capacity of the inner parts of the sorbent and significantly increasing the extraction time. According to the kinetic theory of extraction, the amount of time that is takes to reach $95 \%$ percent of the 
equilibrium ( $\mathrm{te}_{\mathrm{e}, 95 \%}$ ) is given by Equation 7, where B refers to the geometry of which the extraction sorbent is coated, $\delta$ refers is the boundary layer thickness, $b$ is the thickness of the sorbent, $\mathrm{k}^{\prime}$ is the distribution constant of the amount of analyte in the sorbent as compared to the sample matrix, and $D_{s}$ is the is the diffusion coefficient of the analyte in the sample matrix [10]:

$t e, 95 \%=\frac{B * \delta * b * k \prime}{D s}$

The geometric factor that depends on the geometry of the substrate, $\mathrm{B}$, is very important because it accounts for how spread out the substrate is. The higher this number the less the thickness increases as the volume of sorbent is increased, which is critical in extraction kinetics. The time that it takes to reach $95 \%$ of equilibrium (te,95\%) can be decreased in three ways for traditional stationary phases such as pure PDMS: decrease of the B value - this is impractical in most cases and for back extraction with organic solvent increases the volume required; reduction of the $b$ value - this defeats the purpose of trying to extract more by increasing the volume of stationary phase; increase of diffusion of the analyte through the sample matrix ( $D_{s}$ value) by using a method such as stirring or sonication.

An additional important consideration when analyzing the efficient extraction via a stationary phase is that retention of analytes over time is not linear or even close to it. As the time increases, the efficiency of extraction decreases dramatically and ultimately stops when equilibrium is reached. Given this 
phenomenon, the overall effectiveness of the extraction process is most affected by the initial rate of the extraction which is given by Equation 8, where all the variables have been identified before except $A$, the surface area of the sorbent [10]:

$\frac{d n}{d t}=\frac{C a q * D s * A}{\delta}$

Equation 8

The initial rate of extraction is proportional to A. When taking into account all the factors in Equations 7 and 8, it becomes clear that in order to increase sensitivity the sorbent loading capacity needs to be increased by increasing the PCSA, which maximizes all of the sorbent and does not increase the B value in Equation 7.

\subsubsection{Structure of FPSE Media}

Fabric phase sorptive extraction media are composed of a fabric substrate coated with a stationary phase, which is the sorbent (Fig. 15):

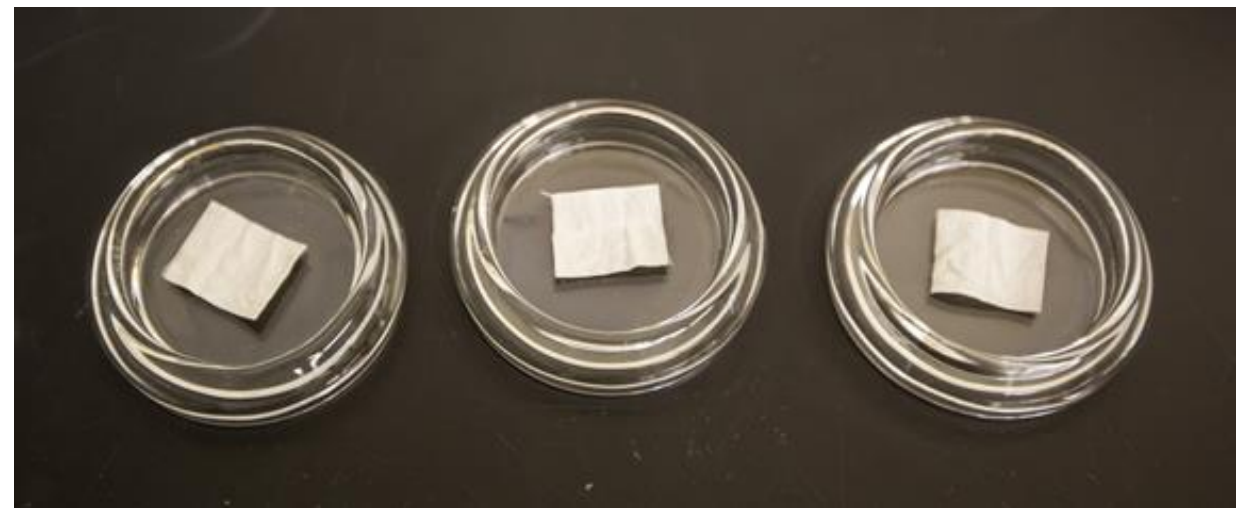

Figure 15: Picture of three $5 \mathrm{~cm}^{2}$ sol-gel PTHF FPSE media. The substrate is cellulose (cotton). 
These simple but highly effective devices are not limited to any specific kind of fabric as the substrate and offer great flexibility because after being coated the fabric can be cut to any desired shape and size. Cellulose has been the most common choice for FPSE media substrate thus far and is very desirable for FPSE media because it has many exposed hydroxyl groups that serve as bonding sites for sol-gel sorbents. As can be seen in Fig. 16 [10], cellulose is a linear polymer of $\alpha$-D-glucopyranose and each of these units has three exposed hydroxyl groups. However, other kinds of fabric have also been used effectively, such as polyester. Sorbents of a wide variety of functionalities have been synthesized for FPSE media, all of them being sol-gel materials. In FPSE media the sol-gel sorbent is always bonded to the substrate, regardless of the specific substrate or sorbent (Figs. 17-20).

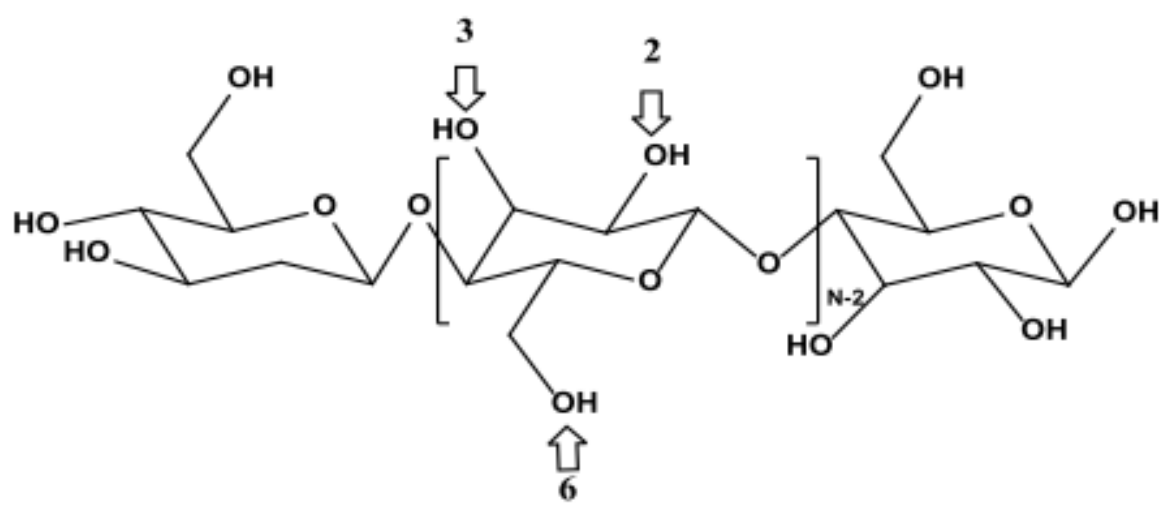

Figure 16: Representation of the chemical structure of the surface of cellulose, showing three exposed hydroxyl groups at positions 2, 3 and 6 of each glucopyranose unit. 


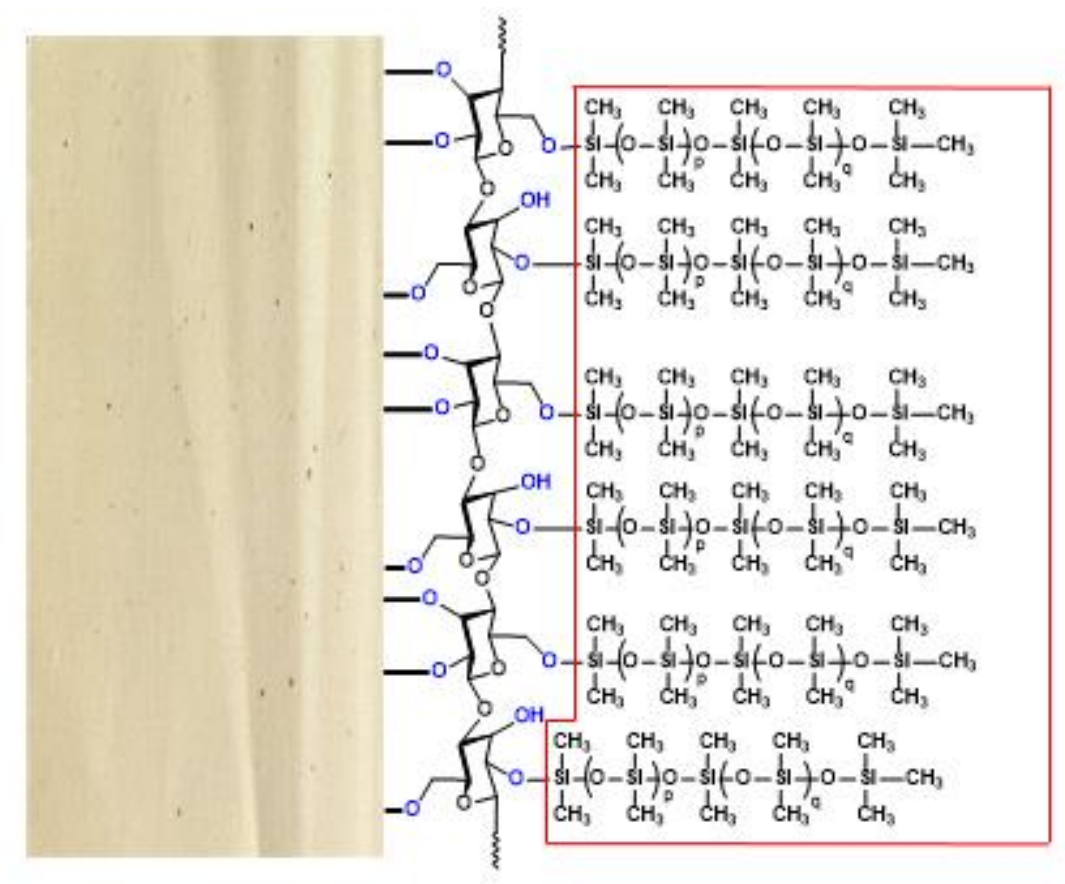

Figure 17: Schematic of sol-gel PDMS FPSE media. The sol-gel network is enclosed in red.

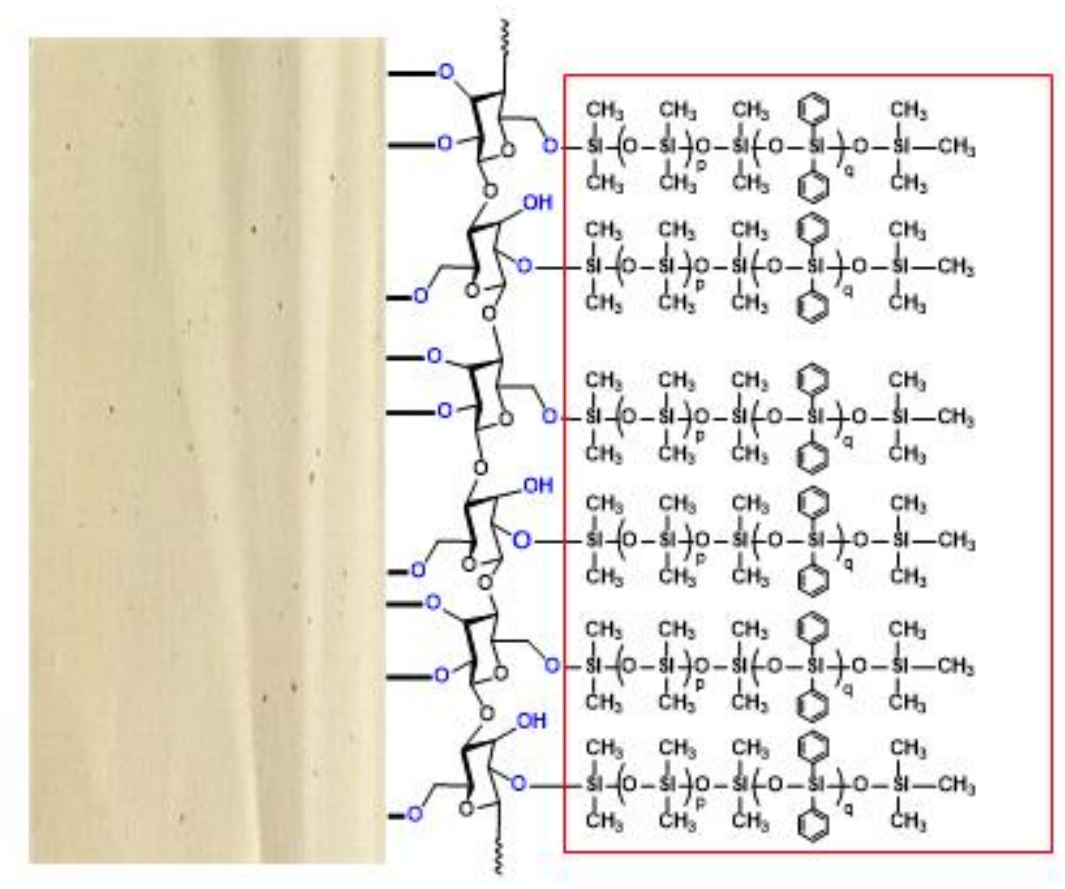

Figure 18: Schematic of sol-gel PDMDPS FPSE media. The sol-gel network is enclosed in red. 


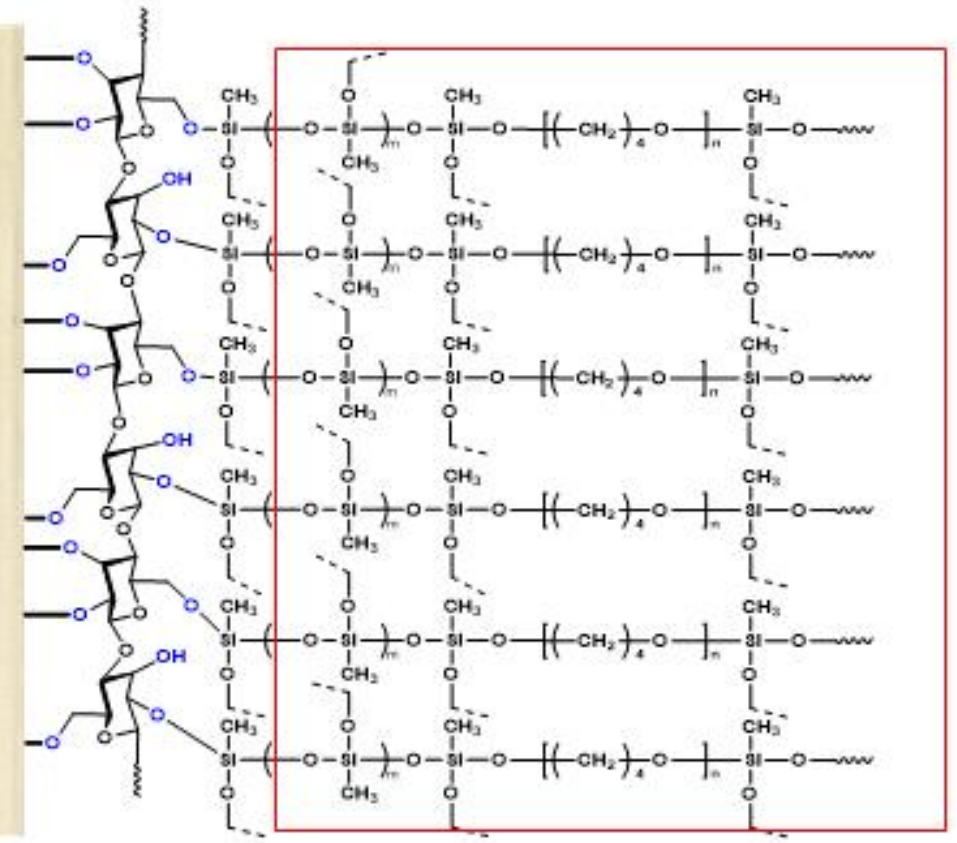

Figure 19: Schematic of sol-gel PTHF FPSE media. The sol-gel network is enclosed in red.

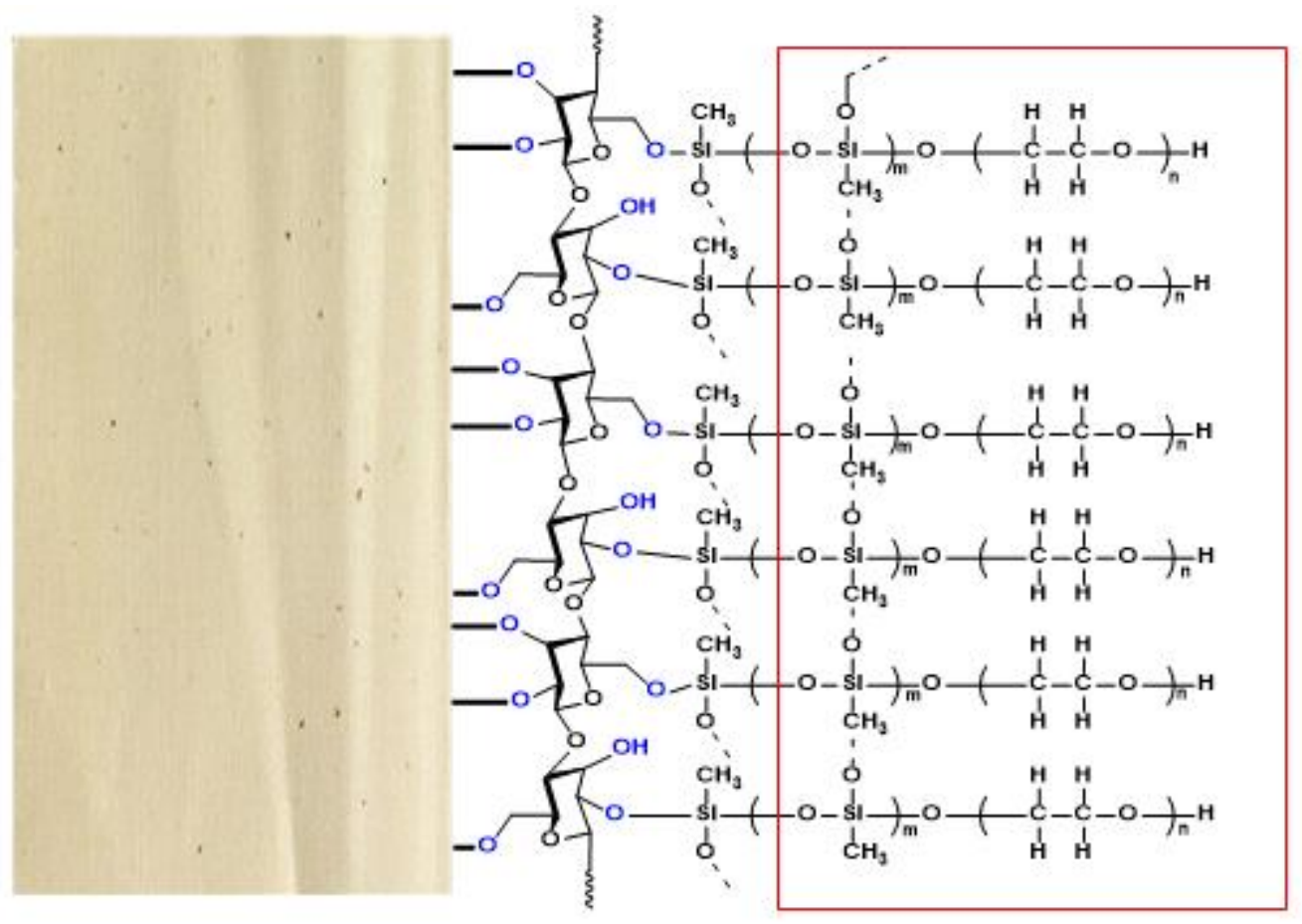

Figure 20: Schematic of sol-gel PEG FPSE media. The sol-gel network is enclosed in red. 


\subsubsection{FPSE Procedure}

The general procedure of direct immersion analysis with FPSE is not very laborious at all, assessed by the fact that and many samples can be extracted from at the same time and much of the time is spent waiting while the sample stirs. It is also quite simple because it only requires five steps: 1 . cleaning by submerging in organic solvent ( 5 minutes); 2. analyte extraction by immersion into liquid sample and subsequent stirring (10 - 50 minutes, which is considered low for complex matrices); 3. back extraction into organic solvent (5 minutes); 4. centrifugation to remove large particles from matrix, which protects the instrument used in the subsequent step (3 minutes); 5 . instrumental analysis (the duration varies widely depending on the analytes and the instrument used).

Figure 21 shows a picture sequence that depicts the procedure of sample preparation by FPSE: 1: Unmodified environmental sample with many particles and microorganisms; 2: Three $5 \mathrm{~cm}^{2}$ FPSE media (sol-gel PTHF sorbent on cellulose substrate) after being cleaned for extraction; 3: FPSE medium held by tweezers; 4: Stirring extraction in progress from unmodified environmental sample; 5: Back extraction in progress; 6: Transfer of back extracted solution to vial for centrifugation; 7: Instrumental analysis by GC-MS; 8: Instrumental analysis by HPLC-UV. Instrumental analysis is not likely to be performed on two different instruments but when the sample preparation is done with FPSE, the back extracted solution allows the possibility of doing so or choosing either option. 

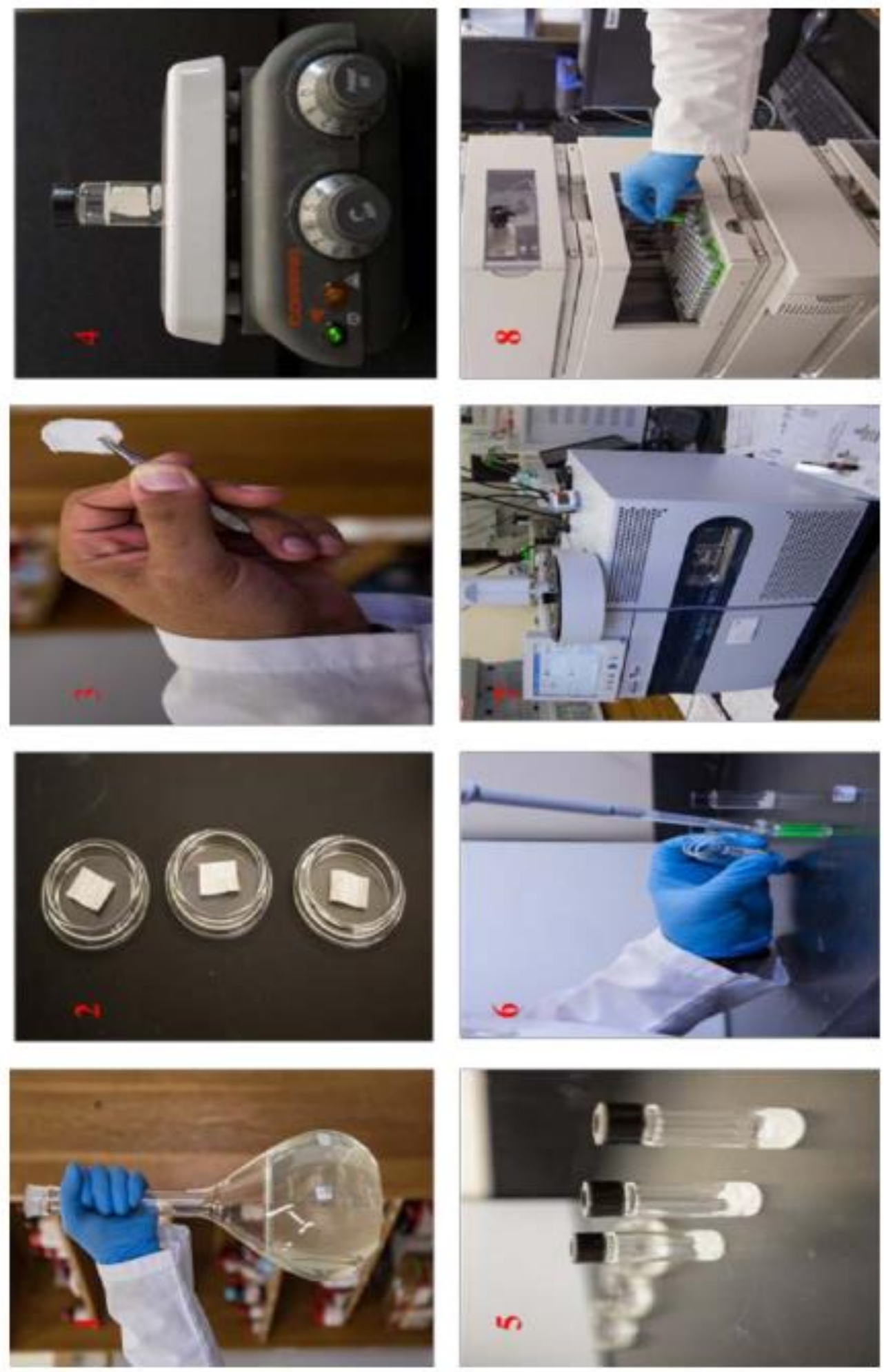

Figure 21: Picture sequence outlining procedure of sample preparation by FPSE. 


\subsubsection{Sol-gel Sorbents}

Sol-gel materials are defined as those that are synthesized by hydrolysis of an inorganic precursor and then undergo poly-condensation to form a threedimensional network $[10,15,16,54,67-70]$. For example, to synthesize the sol solution of a sol-gel material that has PEG as its organic component the following procedure is followed: dissolve $10 \mathrm{~g}$ of $\mathrm{PEG}, 10 \mathrm{~mL}$ methyltrimethoxysilane (MTMS), $20 \mathrm{~mL}$ methylene chloride, $4 \mathrm{~mL}$ trifluoroacetic acid (TFA) (5\% water); vortex for $3 \mathrm{~min}$; centrifuge for $5 \mathrm{~min}$; transfer the clear supernatant (the sol solution) to a clean $100 \mathrm{~mL}$ amber colored glass reaction bottle [10]. The MTMS is hydrolyzed, a reaction catalyzed by TFA. Then hydrolyzed precursors bond to each other and subsequently the growing sol-gel three-dimensional network incorporates the PEG. This process, involving the formation of the sol-gel network is called poly-condensation.

This technology has been found to be a viable pathway to the production of surface coatings $[69,70]$; this is true of many surfaces including but not limited to: stainless steel, titanium, copper, fused silica and fabrics such as cellulose and polyester [67]. Fig. 22 shows the synthetic pathway of sol-gel materials followed by bonding to a surface, forming a revolutionary sorbent for sample preparation [67]. 


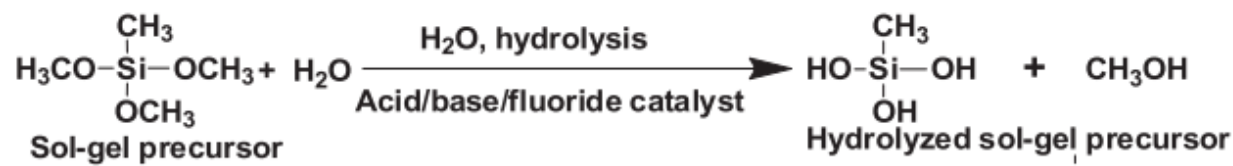
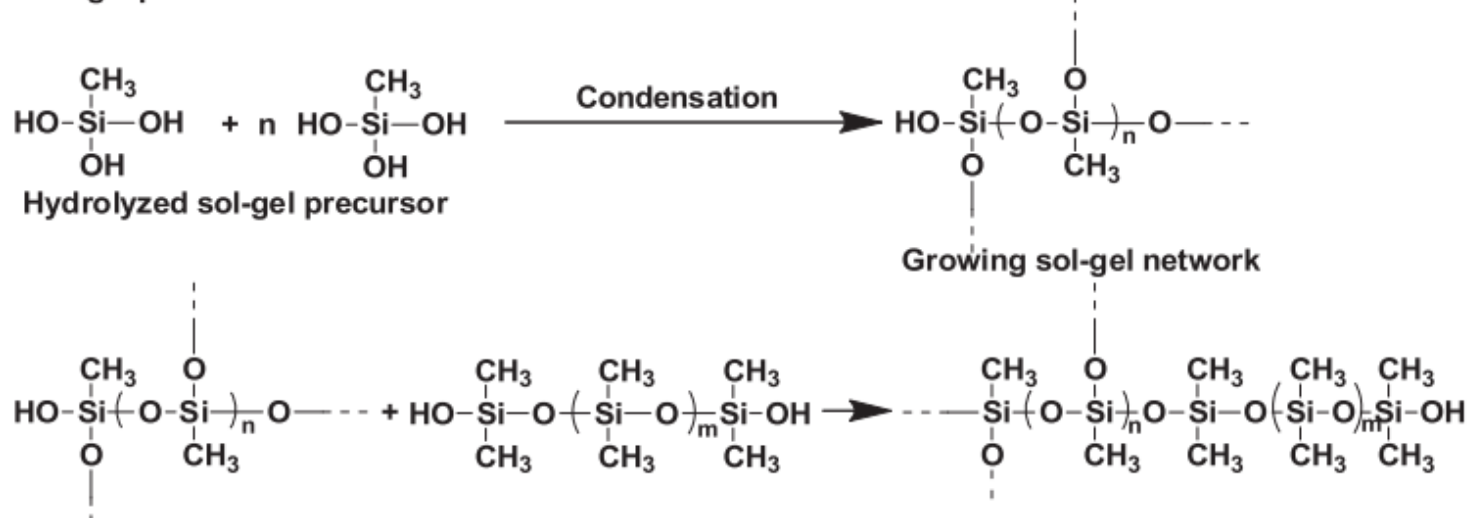

Growing sol-gel network Hydroxy-terminated PDMS Sol-gel network with integrated PDMS

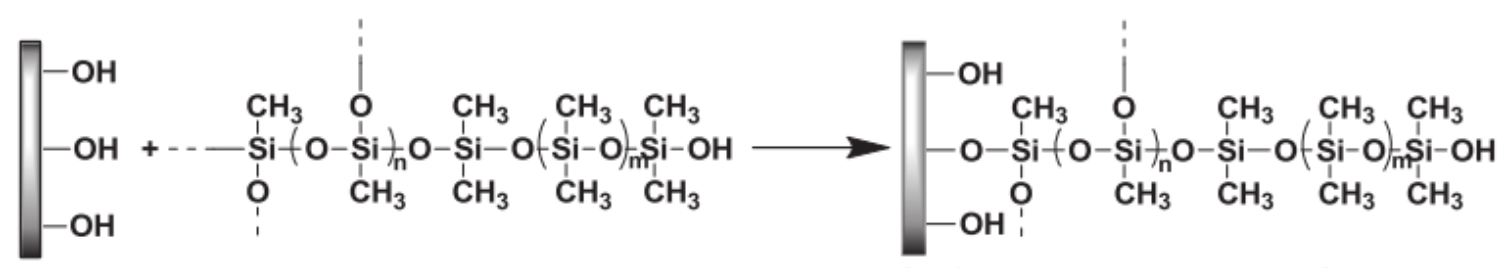

Fiber surface Sol-gel network with integrated PDMS

Surface-bonded sol-gel PDMS coating

Figure 22: Schematic of the major steps in the process of sol-gel synthesis and bonding to a surface, forming a coating that serves as a sorbent.

The applicability of sol-gel materials for coating purposes extends to just about any geometric shapes such as fibers (e.g., traditional SPME) and planar surfaces (e.g., FPSE media) [67]. Additionally, sol-gel materials can be synthesized to be inorganic-organic hybrids by inserting an organic component (such as a polymer) which would become part of the network when the polycondensation phase of the process occurs $[10,15,16,54,67,68]$. Inorganic-organic hybrids are monumental for sample preparation because it, in addition to the fact that there is bonding between sol-gel materials and substrates that it is coated onto for sample preparation, presents the possibility of having an organic component in the material for effective retention of analytes while the material also exhibits 
impressive ruggedness and solvent, chemical and thermal stability $[10,15,16,54,67,68]$. Sol-gel materials are also inherently porous, providing immense PCSA per volume of sorbent when used for sample preparation. Figure 23, a Scanning Electron Microscope (SEM) image of a sol-gel material, shows an example of this great and desirable porosity.

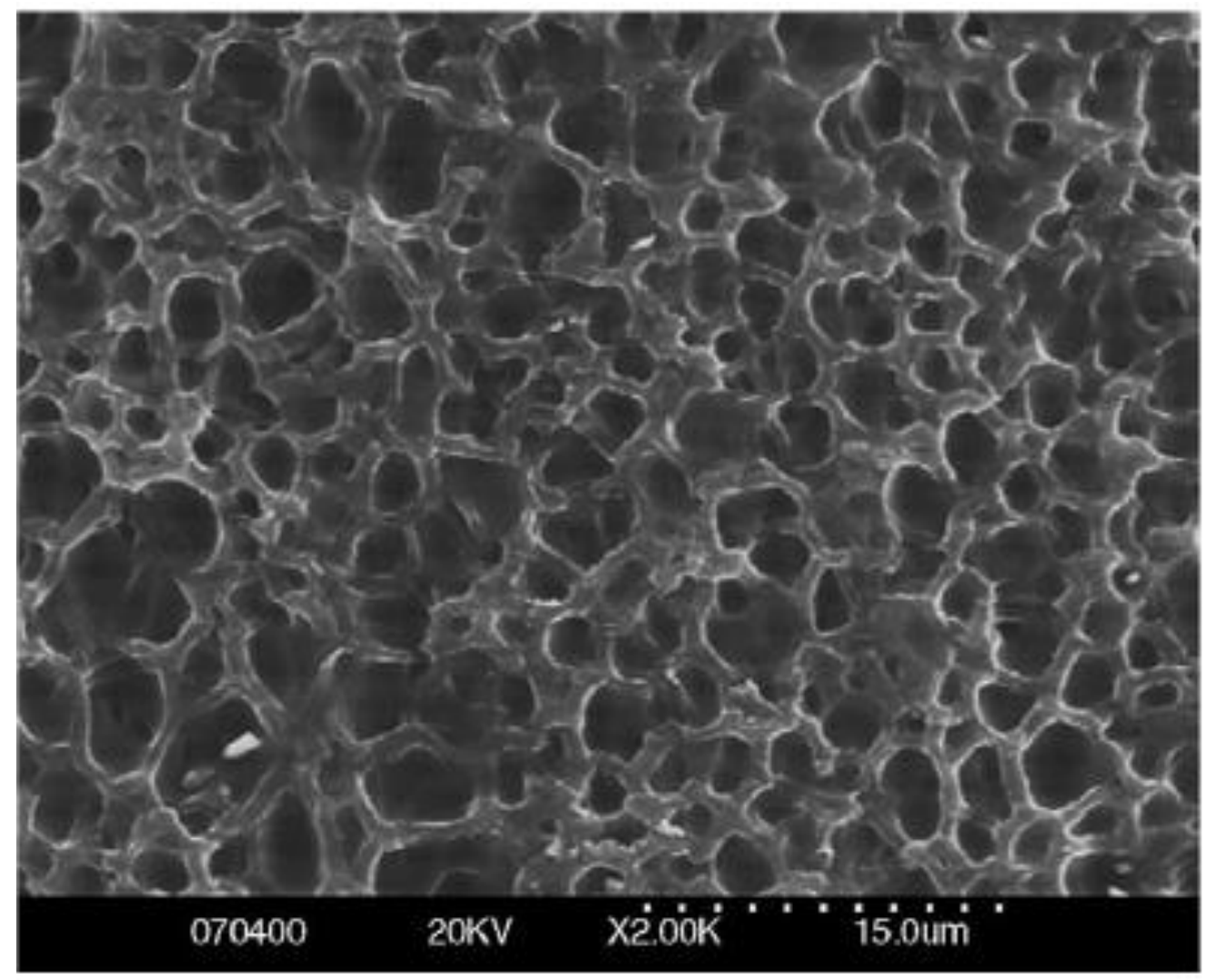

Figure 23: SEM image showing the high porosity inherent in hybrid inorganicorganic sol-gel materials [71].

\subsubsection{Advantageous Characteristics of FPSE}

Necessity and struggle are perhaps the greatest motivators and driving forces behind innovation, as some of the greatest breakthroughs in history have happened as a result of unfortunate or at least undesirable circumstances. Fabric 
phase sorptive extraction is yet another example of an invention that was triggered by need, the need to replace commercially available sample preparation techniques with another that addresses their many vital limitations. Whether it is a consideration that has always been present in analytical chemistry, like sensitivity, or one that is relatively new like following GAC mandates, FPSE presents an unprecedented combination of desirable features for sample preparation. The ability to extract from extremely complex environmental and biological samples such as pond water and milk without prior adulteration is a milestone accomplishment that makes quantitation much more viable and accurate because pretreatment of the matrix would interfere with analytes and decreases the time of analysis. Batch-to-batch reproducibility resulting from sol-gel materials exhibiting uniformity in their synthesis at the molecular level is also exhibited by FPSE as well as excellent solvent, chemical, and thermal stability as a result bonding of the sorbent to the substrate. As illustrated in Fig. 6, FPSE exhibits a high PCSA relative to its size because the structure of FPSE media is spread out over a relatively large surface and the fabric is coated on both sides and the inherent porosity of sol-gel materials; the high PCSA is quite valuable because it results in fast equilibration, high loading capacity and great sensitivity. In addition, FPSE displays field portability without sacrificing merit, applicability to analysis in any kind of instrument (including those that are LC and GC based), and eliminates the need for solvent evaporation and analyte reconstitution by having great sorbent loading capacity 
quickly (low equilibration time) and using small amount of organic solvent for back extraction.

\subsection{Instrumental Analysis}

Over the past few decades there have been achievements that have led to amazing advancement in analytical chemistry instruments, to the extent that they have become a major focus in the ongoing pursuit of the best and most efficient analytical methods possible. For these instruments to yield the best possible results, however, sample preparation is often necessary, especially when quantitation is one of the objectives. Without sample preparation, there are many situations where even the most expensive and acclaimed instruments are inadequate.

\subsubsection{Chromatography}

Chromatography was first developed by a Russian botanist named M. S. Tswett (1872-1919) for separation of colored plant pigments [72]. The name is supposed to have come of the combination of two Greek words: chroma (meaning "color") and graphein (meaning "to write") [72]. While the definition of this technique was given by Tswett himself, several other definitions of it have been accepted and each is more relaxed than its predecessor [72]. In 1974 a special committee of experts in the field of separation science formulated a widely developed definition for chromatography: "A method, used primarily for separation of the components of a sample, in which the components are distributed between two phases, one of which is stationary while the other moves. The stationary phase may be a 
solid, or a liquid supported on a solid, or a gel. The stationary phase may be packed in a column, spread as a layer, or distributed as a film, etc.; in these definitions chromatographic bed is used as a general term to denote any of the different forms in which the stationary phase may be used. The mobile phase may be gaseous or liquid." [73] Even this definition, which was meticulously formulated by highly regarded analytical chemists, does not include the possibility of using a supercritical fluid in chromatography, further indicating that the question "What is chromatography?" may be considered simple but is certainly not necessarily an easy one for anyone to answer.

Whatever the best definition and regardless of how elusive it may be, it is definitely the most significant and commonly used kind of separation technique in analytical chemistry and is used in sample preparation (e.g., SPE) as well as in instrumental analysis. One method of classifying the vast number of chromatographic systems, which often overlap, is by the mobile phase (Fig. 24) [72]. If this is the major classification criterion used, the most common and prevalent kinds of chromatography are Gas Chromatography (GC) and High Performance Liquid Chromatography (HPLC). 


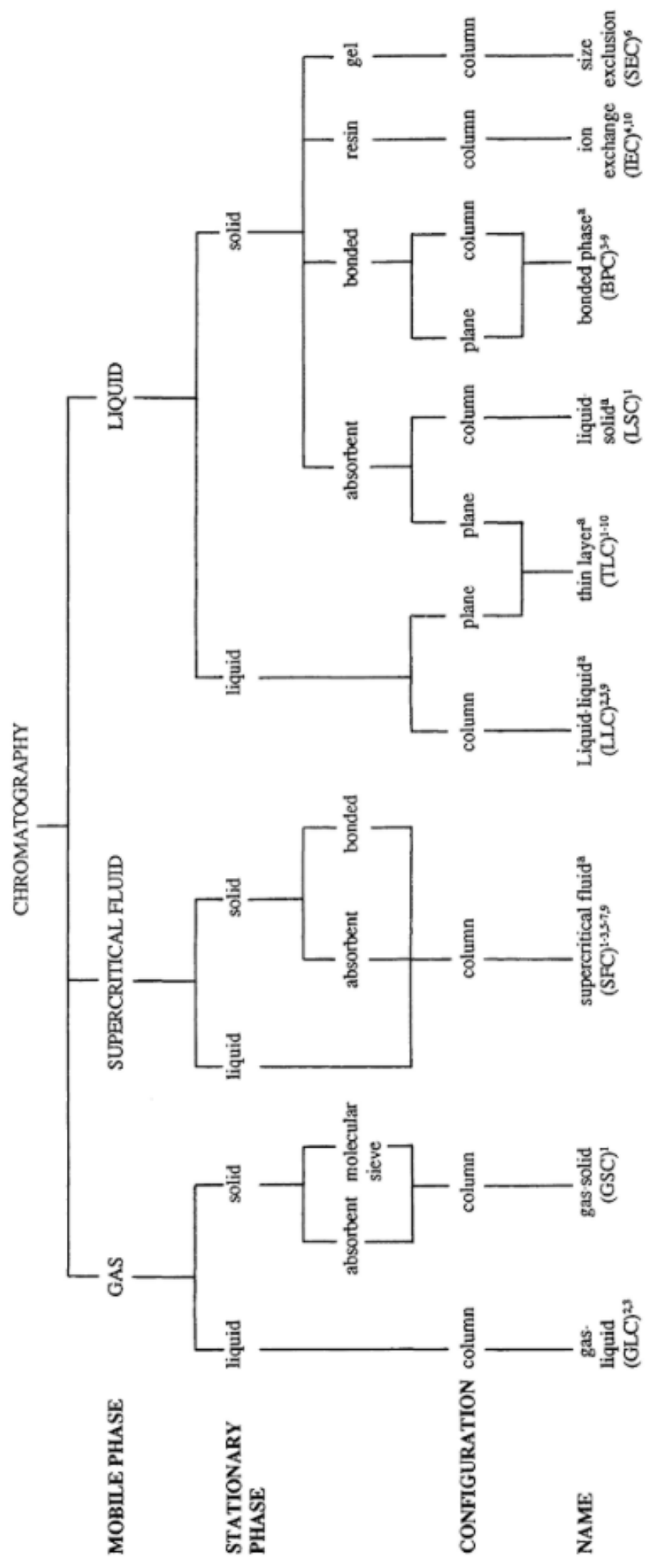

Figure 24: Classification of chromatographic systems by the mobile phase. 


\subsubsection{High Performance Liquid Chromatography}

High performance liquid chromatography uses a stationary phase in a packed column to separate compounds. The theory of the working principle of HPLC is not any different than that of SPE, since the separation in both happens by partitioning with a stationary phase in a packed column, but the performance of HPLC is much greater because its particles are much smaller $(4.6 \mu \mathrm{m}$ in diameter vs $40 \mu \mathrm{m}$ for SPE) [72] which means that there are many more partitioning events in HPLC (can be thought of as individual extractions) between the mobile phase and the stationary phase and therefore the separation power is approximately 4 times better than that of SPE. This, however, only works for samples without any macroparticles. Any particles or even very large molecules such as polymers would clog not only the packed column but possibly even the capillaries of the instrument. Capillaries inherently have a small diameter.

The HPLC analyses that generated the data presented herein were of the reverse phase variety. Reverse phase got its name because it is the opposite of normal phase. It is a term used for when the compounds that are to be separated are in water initially and organic materials (the stationary phase) are used to separate them, whereas normal phase uses aqueous stationary phases to separate compounds mostly in oil-based substances. Even though injected samples after sample preparation with FPSE are always in organic solvent, separating the analytes in the HPLC is still considered reverse phase because when they pass through the column they are carried by an aqueous mobile phase. 
Modern HPLCs consist of a sequence of separate components that communicate with each other and work together. There is typically a tray on top of the instrument to place containers of liquid to use as the mobile phase; then a degasser to eliminate air bubbles from entering the rest of the system and sacrificing the quality and continuity of the results; the next component is a pump (usually a system of four) which is produces the flow of mobile phase throughout all the components at the indicated flow rate; after that an Automatic Liquid Sampler (ALS) which has a robotic arm that picks up the sample vial and takes it to an injection port where a needle withdraws a predetermined amount that is injected and transported by the mobile phase to the next component which contains the packed column with the stationary phase; subsequently there is a detector that indicates which compounds are present and how much of them there is (in the format of a chromatogram, see Fig. 25) and finally the analytes exit the detector and end up in a waste container. Figure 26 shows a simplified diagram of the different major components of a typical HPLC. 


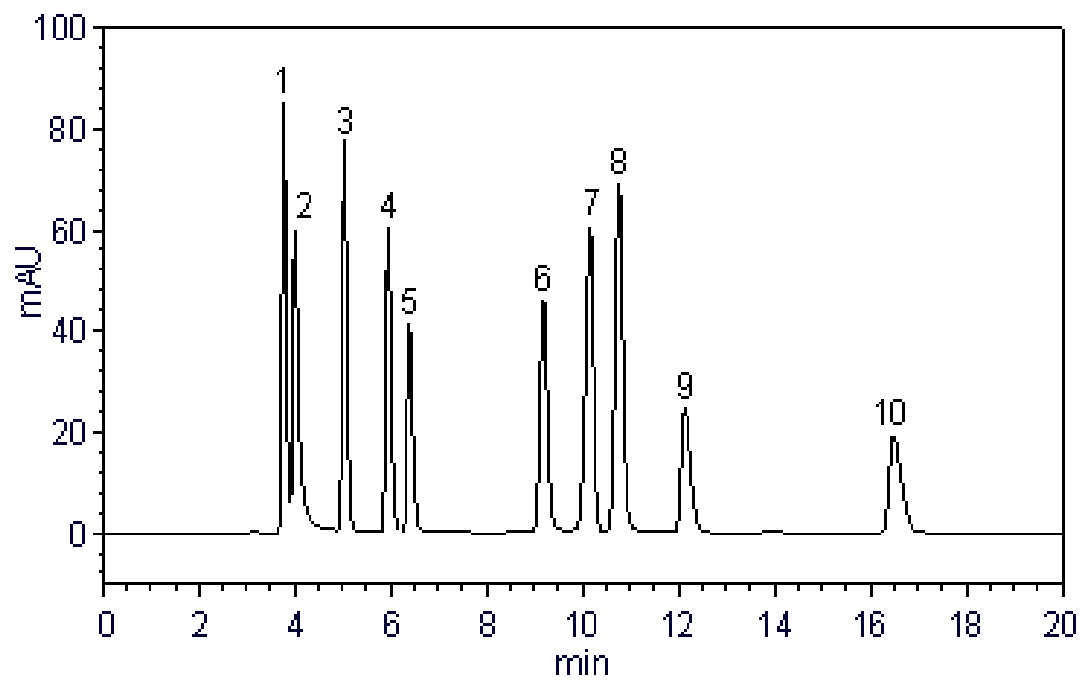

Figure 25: Typical HPLC chromatogram. Each peak represents a specific compound and each compound has a characteristic retention time (time at which the peak is seen from the start of the chromatogram).

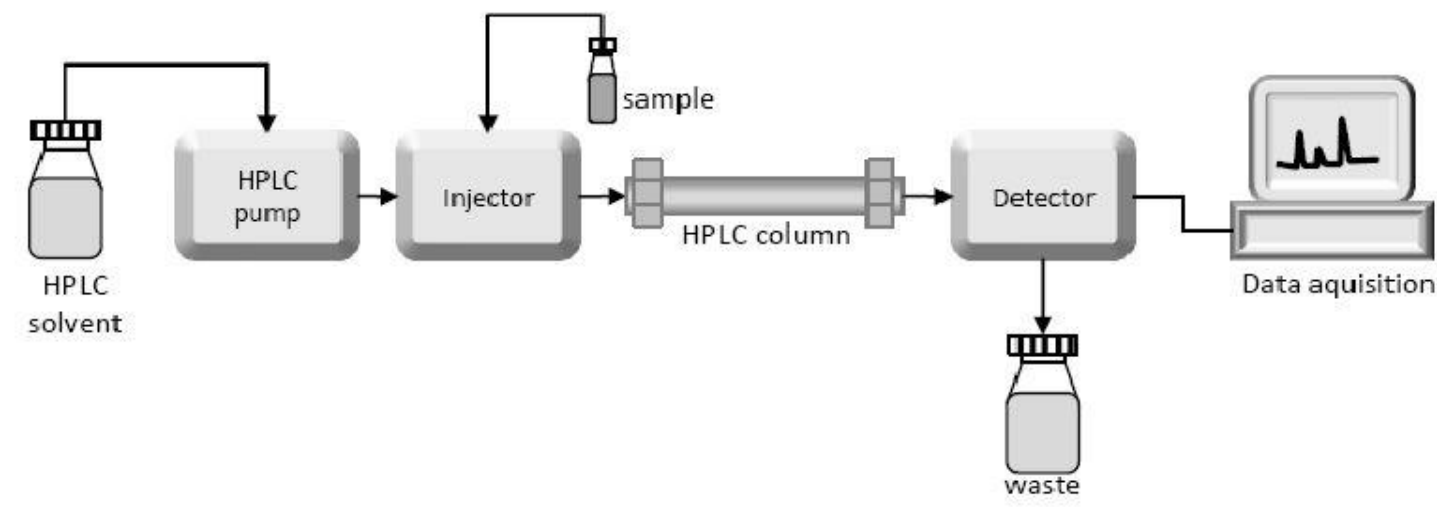

Figure 26: Generic flow diagram of HPLC.

Having a system of four pumps working together is very beneficial because it allows mobile phase solvents to be kept pure. Up to four solvents can be pumped simultaneously and are mixed very conveniently, not to mention that mixing solvents with pumps is more accurate and reproducible than if a person were to do it. Also, HPLC methods often require a gradient (one or more changes to the 
composition of the mobile phase within the method). The separation of the compounds happens when they go through the packed column. These columns have many small silica particles coated with the stationary phase and as the sample is passed through it is constantly partitioned between the mobile phase and the stationary phase until it makes it through the column. Methods are designed such that each analyte takes a different amount of time than the rest to make it through the length of the column due to unique chemical structure and therefore a unique partitioning coefficient.

The performance of an HPLC method is measured primarily by the resolution, which depends on three other essential factors: selectivity, capacity and efficiency. These carry a numerical value but their rudimentary meaning is more easily explained in words. Selectivity represents how far apart two peaks on a chromatogram are from each other. Capacity refers to how long the column retains a specific compound. Efficiency is essentially how sharp a peak is. Those three concepts combine to form the resolution, as seen in Equation 9, where Rs is resolution, $\alpha$ is selectivity, $\mathrm{k}_{2}$ is the capacity of the second of the two peaks and $\mathrm{N}$ is efficiency [72].

$R s=\frac{1}{2} * \frac{\alpha-1}{\alpha+1} * \frac{k^{\prime} 2}{1+k^{\prime} 2} * \sqrt{N}$ Equation 9

In cases where there is only one analyte being considered, resolution is not used and instead the performance of the method is evaluated by the capacity and the efficiency only. In cases where two peaks are symmetrical and of Gaussian 
shape, the resolution can be calculated by using Equation 10, where $\operatorname{tr}_{1}$ and $\operatorname{tr}_{2}$ is the retention times of the peaks and $\mathrm{wb}_{\mathrm{b} 1}$ and $\mathrm{wb}_{\mathrm{b}}$ are the widths of the base of the two peaks [72]:

$R s=\frac{2 *(\operatorname{tr} 2-\operatorname{tr} 1)}{w b 1+w b 2}$

Equation 10

\subsubsection{Ultra-Violet/Visible Detector}

There are many kinds of detectors that can be used sequentially to HPLCs, each with their own advantages and disadvantages. One such kind is UltraViolet/Visible (UV/vis or UV) Detector. This kind of detector is useful for quantitation of compounds that absorb waves in the range of $190-800 \mathrm{~nm}$. Most of the detection takes place toward the lower end of that range which for organic molecules is the result of conjugation (alternating double and single carboncarbon bonds).

Light from a UV lamp is passed through a filter or grating (to select the best possible wavelength) and then to a cell through which the mobile phase flows whether it is carrying analytes or not [72] (Fig. 27). The mobile phase must not have any absorbance at the wavelength being used because if it does it would create interference and even if the method is isocratic it could lessen the sensitivity. The admitted light that passes through the cell is measured by a photodetector [72]. The photodetector produces a current that is converted to a voltage by an amplifier. The entire system is connected to a computer with 
operating software corresponding to the instrument. Both the instrument and software are made available by the same company.

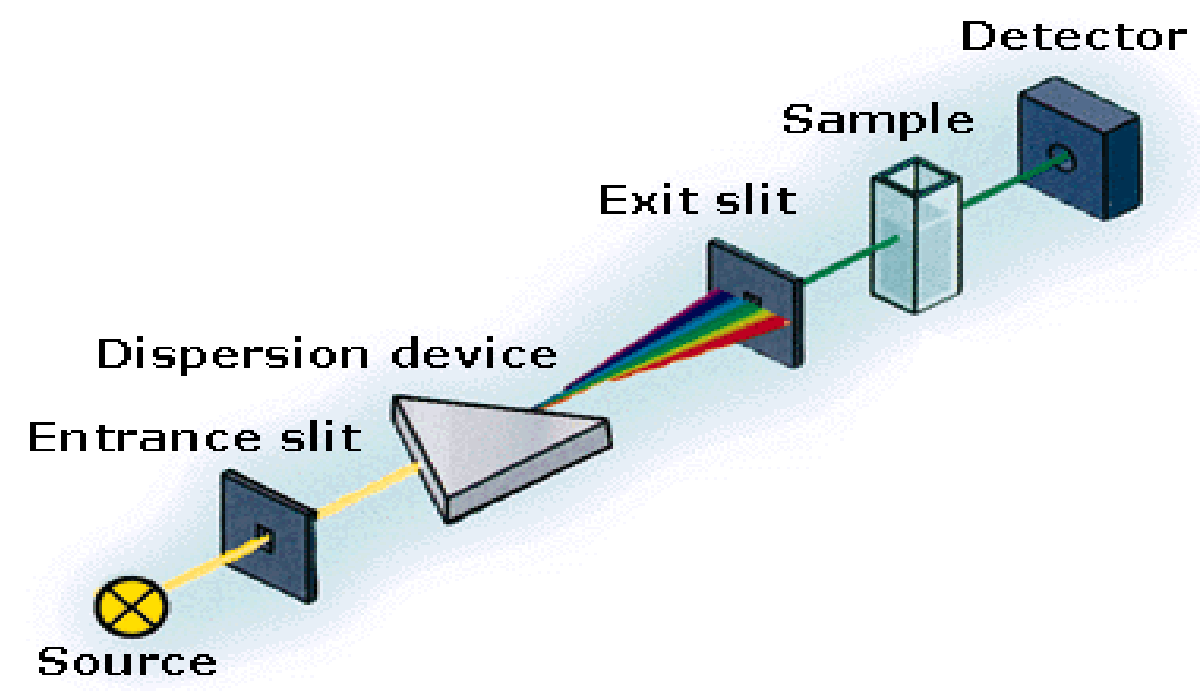

Figure 27: General schematic of generic UV detector.

When there is a drop-off in the transmittance of light, which would be due to absorbance of the light by a compound, there is also a decrease in the resulting current and a peak is produced in the chromatogram. The absorbance is proportional to the concentration (and therefore the absolute amount) of the compound that is absorbing the light, which can be seen from the Beer-Lambert Law:

$A=\log \frac{P 0}{P}=\varepsilon * b * C$ Equation 11

The variable $A$ is absorbance, $\mathrm{P}_{0}$ is the intensity of the light focused onto the cell, $P$ is the intensity of the light transmitted, $b$ is the optical path length through the cell measured in centimeters, and $\mathrm{C}$ is the concentration of the absorbing compound. 


\subsubsection{Mass Spectrometry}

Mass spectrometry (MS) may be the single most important kind of technology in analytical chemistry and one of the most important in any science because it is greatly useful for identification of analytes with great sensitivity. Quite frankly, mass spectrometry is a testament to the great potential of the human brain for innovation and using simple concepts to create a product greater than the sum of its parts.

Mass spectrometry methods preceded by chromatography have been proven sensitive beyond anyone's expectations, down to attomole or even zeptomole levels [74-76]. Having progressed at an incredible pace over the last two decades, entirely new instruments have been designed and built that outperform those they replace. The principles of mass spectrometry are quite simple. Gas phase ions are broken up, yielding a pattern of fragments measured using the mass-to-charge ratio $(\mathrm{m} / \mathrm{z})$ that is characteristic of the original ion. The responses in the pattern, called a mass spectrum, are proportional to the abundance of the ion corresponding to each $\mathrm{m} / \mathrm{z}$. As it happens, however, mass spectrometers and the theories that drive their design can become very complex as attempts are made to meet very demanding analytical challenges. If the mass is expressed in atomic mass units $\left(1 \mathrm{u}=1.660540^{*} 10^{-27} \mathrm{~kg}\right)$, and the charge of each electron is represented as a whole number for each electron $(1 \mathrm{e}=1.602177 \mathrm{C})$, then the dimensions for $\mathrm{m} / \mathrm{z}$ are $\mathrm{u} / \mathrm{e}$ [76]: $1 \mathrm{Th}=1 \mathrm{u} / \mathrm{e}=1.036426^{*} 10^{-8} \mathrm{~kg} / \mathrm{C}$, where Th is the symbol for a unit called Thompson that was created for practical purposes [76]. 
Figure 28 shows an overly simplified but technically correct diagram of a generic mass spectrometer.

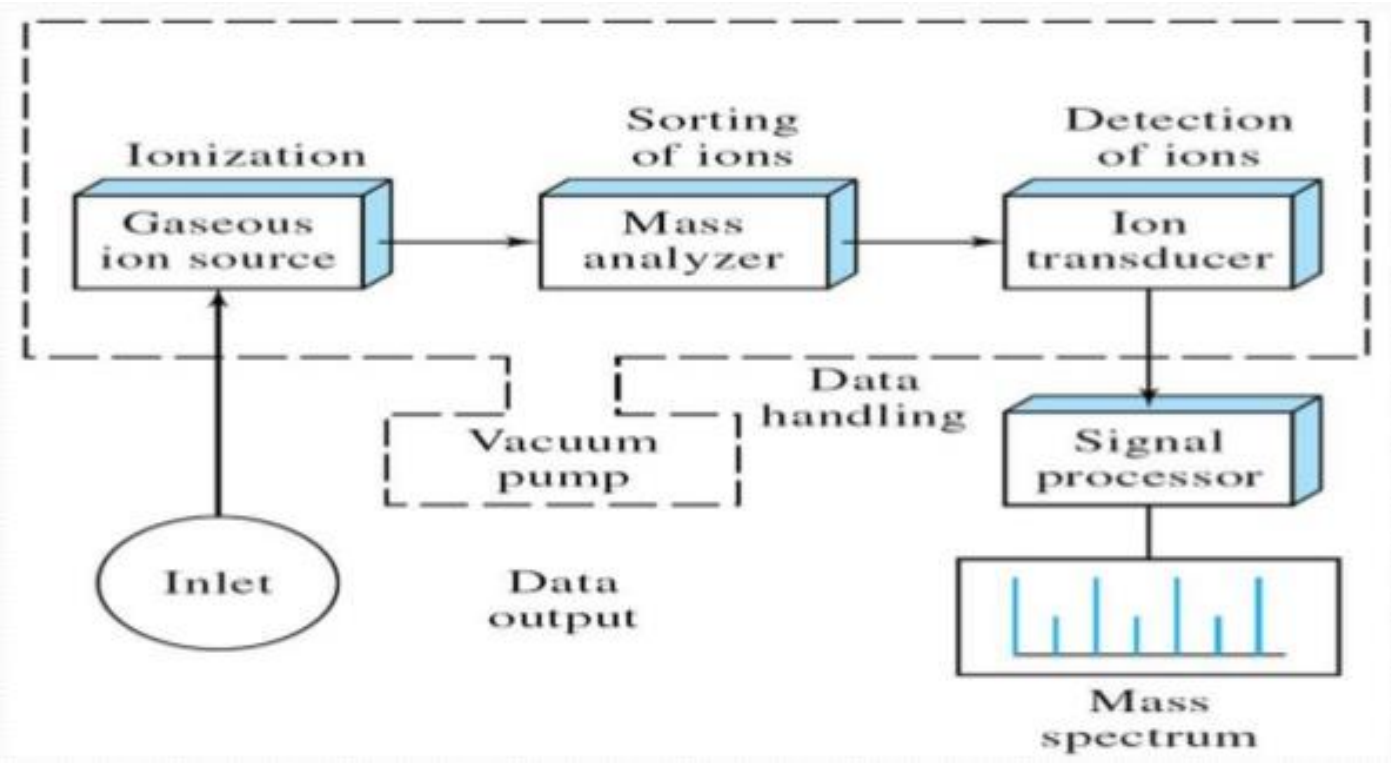

Figure 28: Diagram of generic mass spectrometer.

The first step in mass spectrometry is to introduce the sample, which sounds trivial but in certain cases can be problematic. Immediately after that, ions are produced in the gas phase because most of the time analytes are not already ionic and in the gas phase. This production of ions is done with an ionization source. There are many different ionization sources available, each with its own set of capabilities, attributes, and deficiencies. During many years, ionization sources were only functional in a high vacuum, but presently some have been developed that work under atmospheric pressure. Once the sample is turned into ions in the gas phase by the ionization source, the resulting fragment ions (typically a mixture of the molecular ion and fragments of it) are sorted per their $\mathrm{m} / \mathrm{z}$ by mass analyzers. There is a great variety of mass analyzers available for 
different specific goals, just like there are many different ionization sources available. Once the ions are sorted they are detected, the signal processed by a computer and the mass spectrum generated. At this point the spectrum is analyzed according to the goal of the analysis, which usually is to identify and quantify analytes.

\subsubsection{Electrospray lonization}

Electrospray lonization (ESI) is one of the most important ionization techniques because it is the best option for coupling liquid chromatography to mass spectrometry (LC-MS). Originally proven to be effective for analysis of proteins,

ESI was recognized as a successful technology because Fenn et al. showed that it produced multiply charged ions from each protein molecule $[76,77]$. Eventually accepted as a viable ionization source for small molecules as well as large ones such as proteins and polymers, ESI not only makes coupling of HPLC to MS quite practical, it also exhibits great sensitivity [76].

Electrospray ionization is a great name for this technology because it is a selfexplanatory term. A liquid, such as the mobile phase of an HPLC after leaving the column, is subjected to a strong electric field under atmospheric pressure as it passes through a capillary at a rate from 1 to $10 \mu \mathrm{L} / \mathrm{min}[76,78-82]$. A potential difference of $3-6 \mathrm{kV}$ is applied between the capillary and the counter-electrode, which is no more than $2 \mathrm{~cm}$ apart to create the electric field (in the order of $10^{6}$ $\mathrm{V} / \mathrm{m})[76]$. Figure 29 presents a schematic of ESI that uses skimmers for ion focalization and heated nitrogen gas for desolvation, which is the removal of any 
remaining solvent molecules [76]. Alternatively, a heated capillary can be used for desolvation (Fig. 30) [76].

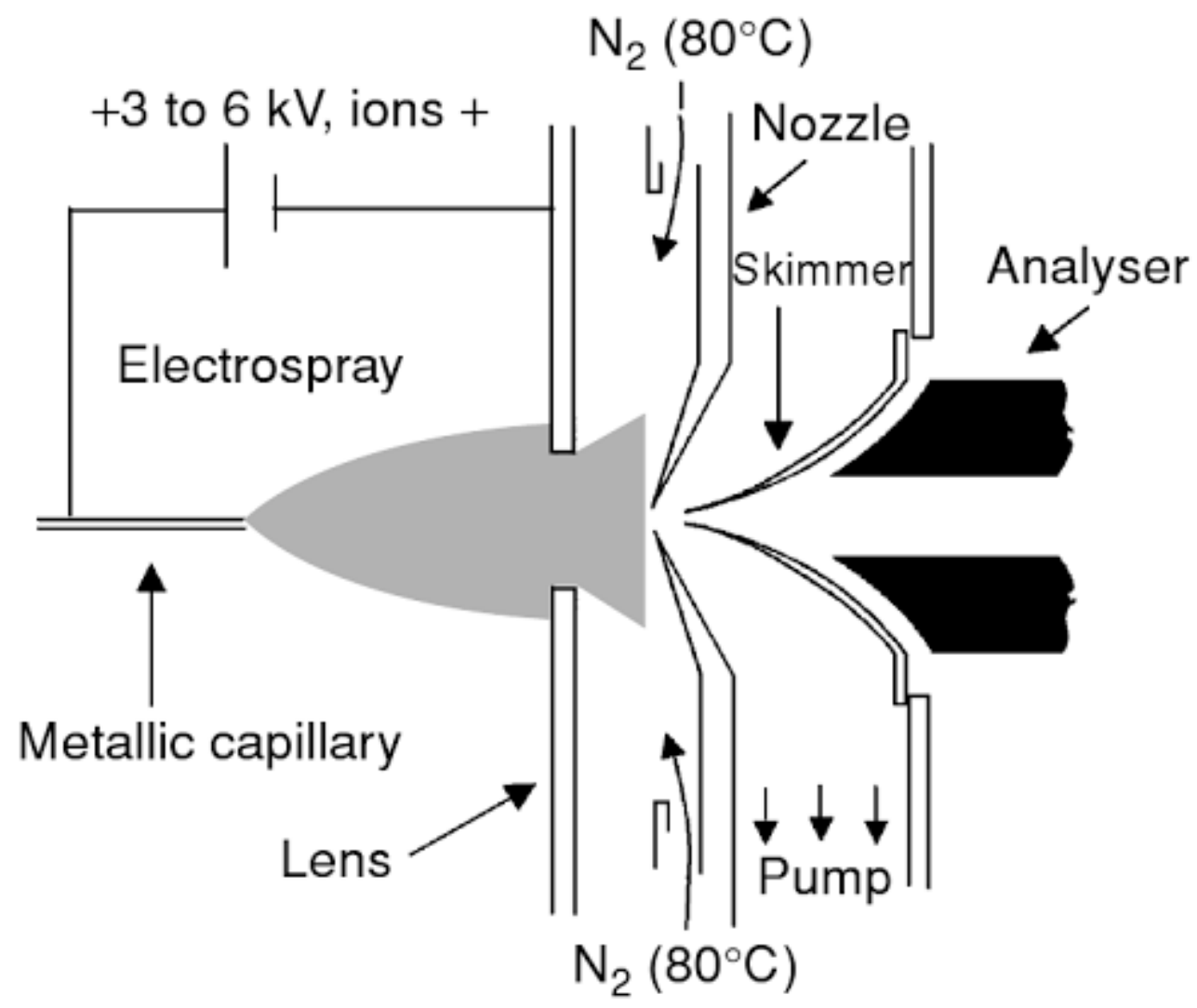

Figure 29: Diagram of ESI source with skimmers for ion focalization and heated nitrogen gas for desolvation.
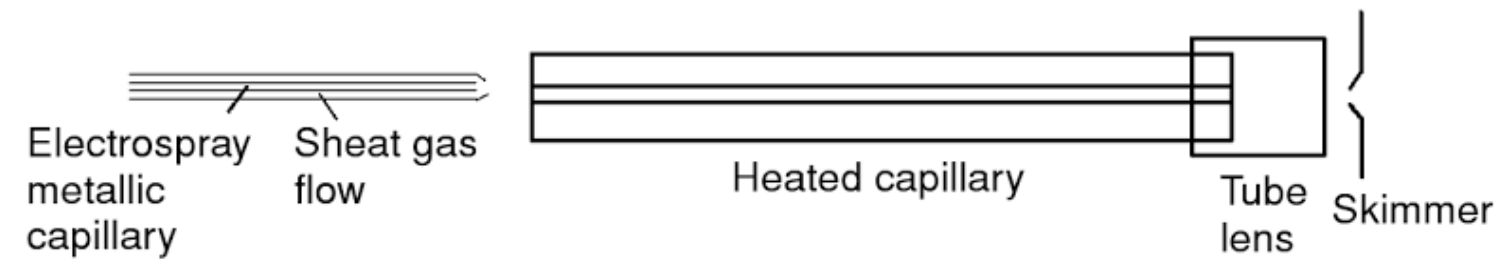

Figure 30: Diagram of ESI source with heated capillary for desolvation.

Because of this electric field there is an accumulation of charge at the end of the capillary that leads to the formation of a drop (about $1.5 \mu \mathrm{m}$ in diameter depending on the solvent) at the tip [76]. The surface tension of this drop is 
overcome by the electric field and approximately 20 much smaller (about $0.1 \mu \mathrm{m}$ in diameter), highly charged droplets are formed. The droplets are a spray on a small scale, which is why the name electrospray makes sense for this approach to ionization, it uses an electric field and creates a miniature spray in the process of ionizing analytes. The magnitude of the voltage required to overcome the surface tension depends on the solvent used; as the voltage is increased the drop at the tip of the capillary elongates and eventually breaks apart (Fig. 31). When elongated to the point where the smaller droplets are released, the resulting shape is called a Taylor cone.
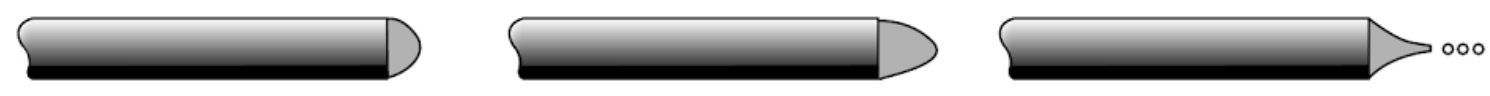

Figure 31: Illustration of the effect of the magnitude of applied voltage on a drop of liquid in ESI. When the voltage is low (left) the drop is spherical, at a higher voltage it elongates (middle) until the pressure is greater than the surface tension (at the onset voltage) and smaller droplets are released.

After the highly charged droplets are released, the solvent evaporates and the electric field accumulates on the surface causing both positive and negative ions to move there and desorb [76]. This process needs to be considered in every analysis of multiple compounds, especially if they differ greatly from each other, because more lipophilic ones will be more abundant on the surface of the droplet and can mask the presence of those that are less lipophilic, which will be less abundant [76]. Another important consideration is that a volatile electrolyte is needed in the solvent to have a stable spray in ESI, not to exceed $10^{-3} \mathrm{M}$ in 
concentration [76]. A concentration low enough that commonly used solvents such as methanol and acetonitrile already contain enough, without any addition.

One great characteristic of ESI is the superb sensitivity that it produces. It is fascinating that the sensitivity in electrospray ionization directly proportional to concentration and not absolute quantity of a compound. This distinction is considerable, which is made evident by the recent modifications of this technique that resulted in the production of two derivatives of it called microelectrospray $(\mu \mathrm{ESI})$ and nanoelectrospray (nESI) [76]. Microelectrospray and nanoelectrospray ionization use smaller capillaries than tradition ESI, making the resulting drop smaller and everything else after that [76]. These new technologies have been found to be more sensitive than traditional ESI while also decreasing noise and interference effects [76].

\subsubsection{Triple Quadrupole Tandem Mass Analyzer}

Two mass analyzers commonly used with liquid chromatography are quadrupoles and ion traps. Five criteria are used to assess the performance of mass analyzers: mass range limit (the maximum mass that the instrument is compatible with per unit charge); analysis speed (rate of measuring over a mass range); transmission (quantity of ions that reach the detector over the quantity that enters the analyzer); mass accuracy is the accuracy of the $\mathrm{m} / \mathrm{z}$ provided by the analyzer, measured by how close it is to the theoretical $\mathrm{m} / \mathrm{z}$; resolution is also referred to as resolving power and has proven quite difficult to define as there is debate over what the technical definition should be [76]. While the competing 
definitions tend to be complex and confusing, it is basically a measure of how much ions are separated in time. Ideally the valley between two peaks is $10 \%$ or less than the height of the shorter peak of the pair; Fig. 32 shows this concept and the difference between acceptable and unacceptable resolution [76].

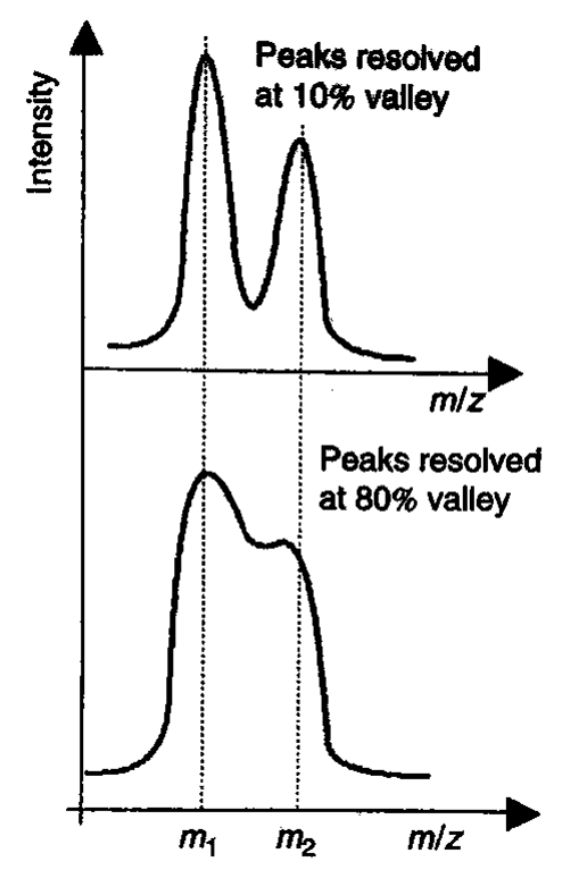

Figure 32: Two situations where the concept of looking at resolution per the height of the valley between the two peaks is illustrated.

While these five criteria for assessing the performance of mass analyzers are all important in certain situations, the one most relevant for the research presented herein is the resolution. When stating the performance attributes of an analyzer the resolving power is given by Equation 12:

$R=\frac{m}{\Delta m}$

Equation 12 
Quadrupoles are composed of two pairs of perfectly parallel metal rods that use a combination of alternating current (AC) and direct current (DC) potential to filter ions (Fig. 33).

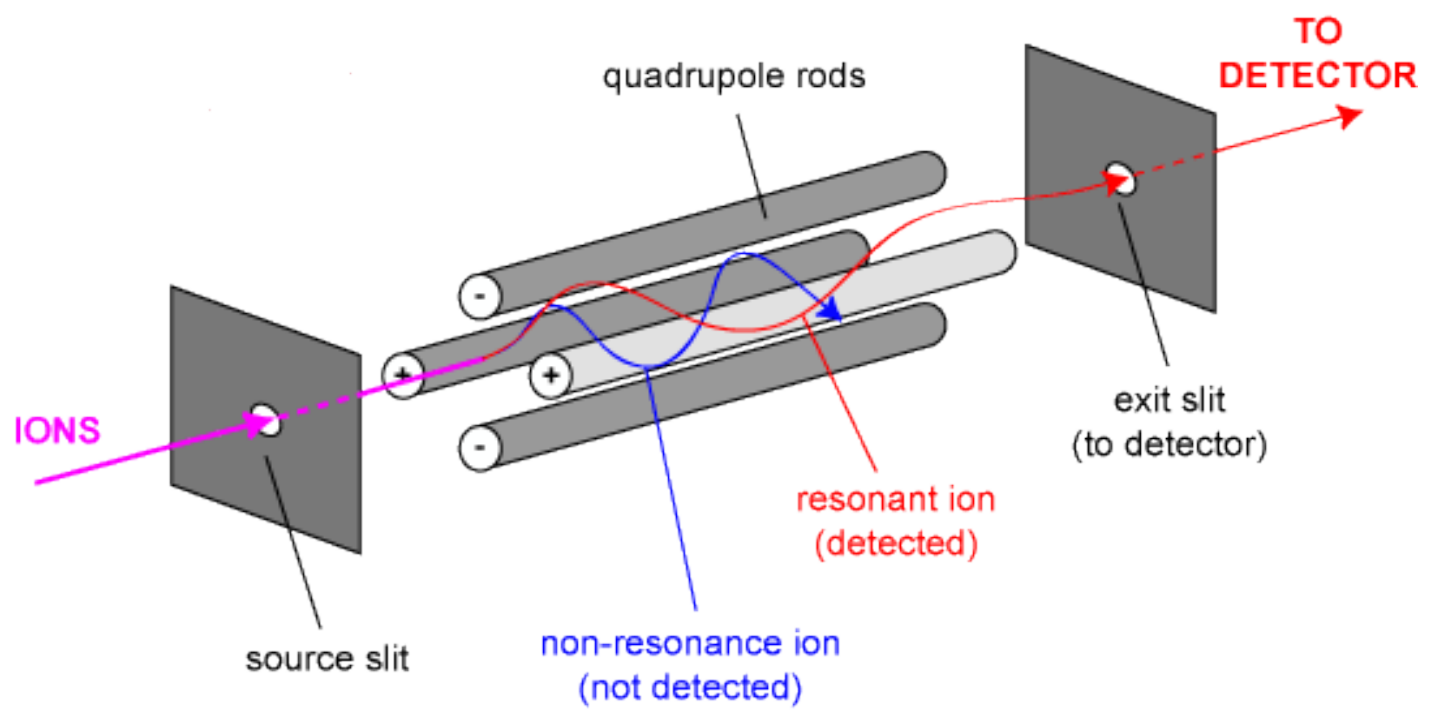

Figure 33: Schematic of a quadrupole mass spectrometer, including the path of an ion transmitted to the detector (resonant ion) and another that is expelled before reaching the detector (non-resonant ion).

The rods are either round or octagonal or hyperbolic; two of the rods, opposite to each other, use DC potential current while the other two use AC. All ions are affected by DC potential, while AC potential affects only light ions, with the threshold depending on the magnitude of the potential. Quadrupoles can be used a low pass mass filters, high mass filters, or to filter all ions except for a very narrow mass range. For positive ions, for example, a negative potential on the DC rods attracts all ions. The AC potential from the other two rods would then be positive and repel the light ions. This results in transmission of the light ions along the center of the four rods through to the detector because the effect of 
each voltage on these ions is cancelled out by that of the opposite pole. The heavy ions, on the other hand, are expelled (Fig. 34) [83].

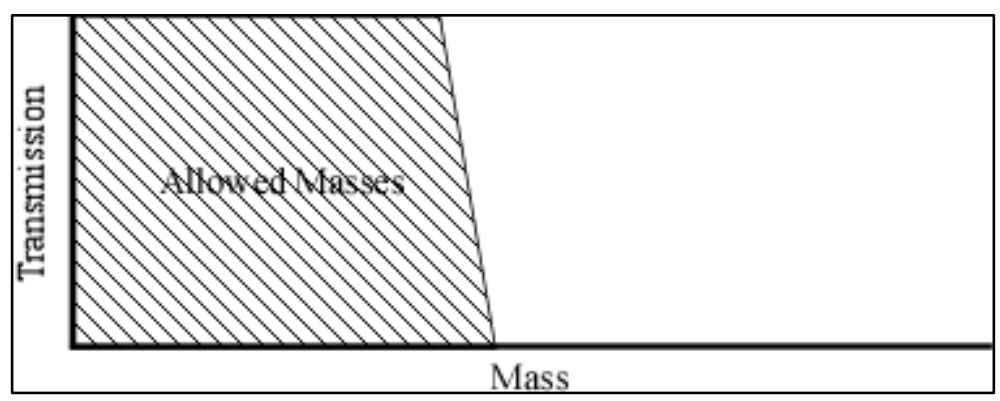

Figure 34: Low pass mass range representative diagram.

Alternatively, quadrupoles can also be high pass mass filters, which is useful if the goal is to expel lighter ions and keep heavier ones. In this case the DC voltage is positive, repelling all ions since these are positive too. The DC voltage being positive means that the $\mathrm{AC}$ voltage is negative, attracting the light ions. Because the light ions are the only ones that are being attracted one way or the other, these escape the region between the poles before transmission to the detector. The heavy ones go through the length of the quadrupole and into the detector because they are only affected by forces keeping them in the center (see Fig. 35) [83]:

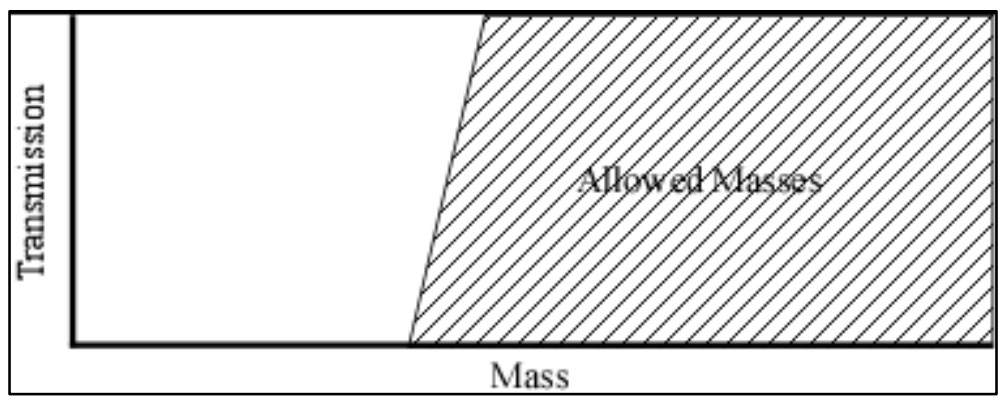

Figure 35: High pass mass range representative diagram. 
Looking at a large range of low or high mass has very limited practical value, however. Mostly, quadrupoles are used to filter out all ions outside of a narrow mass range. The filtering is done by combining the low pass mass filter application with the high pass mass filter (Fig. 36) [83]. Depending on the desired $\mathrm{m} / \mathrm{z}$, the instrument adjusts the magnitude of the $A C$ and $D C$ voltages. The selected narrow mass range can be changed fast enough to scan through a large range of mass ranges, creating separation in space.

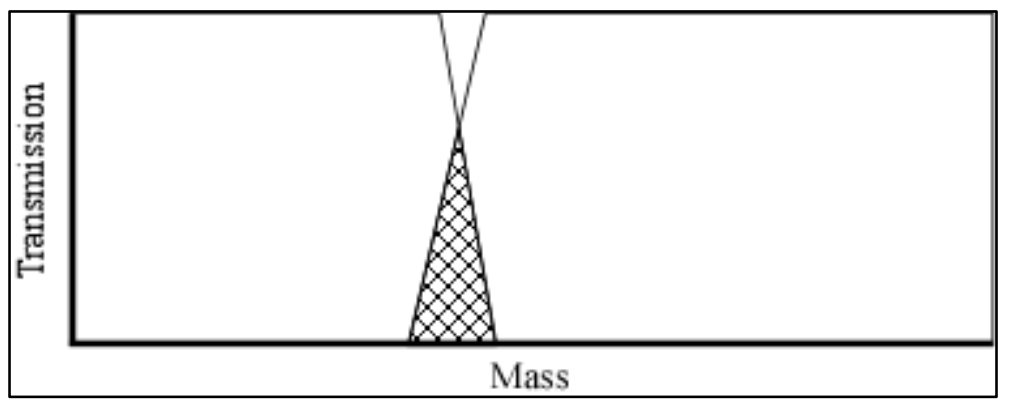

Figure 36: Narrow mass range representative diagram. The potential applied to each pair of quadrupole rods are given by Equations 13 and 14 , respectively [76]:

$$
\begin{array}{lr}
\Phi 0=(U-V * \cos (\omega * t) & \text { Equation } 13 \\
\Phi 0=-(U-V * \cos (\omega * t) & \text { Equation } 14
\end{array}
$$

where $\Phi_{0}$ represents the potential applied to the rods, $\omega$ is the angular frequency, $\mathrm{U}$ is the potential that results from $\mathrm{DC}$ and $\mathrm{V}$ is the potential from AC. Typically, $U$ ranges in magnitude from 500 to $2000 \mathrm{~V}$ and $\mathrm{V}$ from 0 to $3000 \mathrm{~V}$ [76]. The forces 
induced by the electric fields generated due to the potential applied to the rods are given by Equations 15 and 16:

$F x=m * \frac{d^{2} x}{d t^{2}}=-z * e * \frac{\partial \Phi}{\partial x}$

Equation 15

$F y=m * \frac{d^{2} y}{d t^{2}}=-z * e * \frac{\partial \Phi}{\partial y}$

Equation 16

where $F_{x}$ and $F_{y}$ are the forces along the $x$ and $y$ planes of motion, respectively, $\mathrm{m}$ is mass and $\Phi_{(x, y)}$ is given by Equation 17:

$\Phi(x, y)=\frac{\left(\mathrm{x}^{2}-\mathrm{y}^{2}\right) *[\mathrm{U}-\mathrm{V} * \cos (\omega * \mathrm{t})]}{r 0^{2}}$

Substitution of Equation 17 into 15 and 16, respectively, followed by differentiation and rearrangement yield Equations 18 and 19:

$\frac{d^{2} x}{d t^{2}}=-\frac{2 * Z * e}{m * r 0^{2}} *[U-V * \cos (\omega * t)] * x$

Equation 18

$\frac{d^{2} y}{d t^{2}}=-\frac{2 * Z * e}{m * r 0^{2}} *[U-V * \cos (\omega * t)] * x$

Equation 19

These are the Paul Equations [84], which are a mathematical representation of the trajectory of ions along the $\mathrm{x}$ and $\mathrm{y}$ planes.

As can be seen in Fig. 37, three quadrupoles can be used consecutively in the same instrument, complementing each other synergistically by allowing multiple reaction monitoring (MRM). Such mass spectrometers are called triple 
quadrupoles (QQQ). During MRM the first quadrupole is used as a filter to make sure that only a selected ion makes it to the second quadrupole, which is used as a collision cell. Here the ions are fragmented by being bombarded with a gas, a process known as Collision Induced Dissociation (CID). The product ions then enter the third quadrupole, where they are again sorted and only those of a specified $\mathrm{m} / \mathrm{z}$ are measured. These are called transitions, because the operator is looking first at the presence and quantity of an ion at a given $\mathrm{m} / \mathrm{z}$ and then at a different one that that ion transitioned to. For example, if the analyte is bisphenol A, the transition $227.050 \rightarrow 133.200 u$ is appropriate because $227.050 u$ is the exact mass of bisphenol A and $133.200 \mathrm{u}$ is the exact mass of a predominant product ion of bisphenol A.

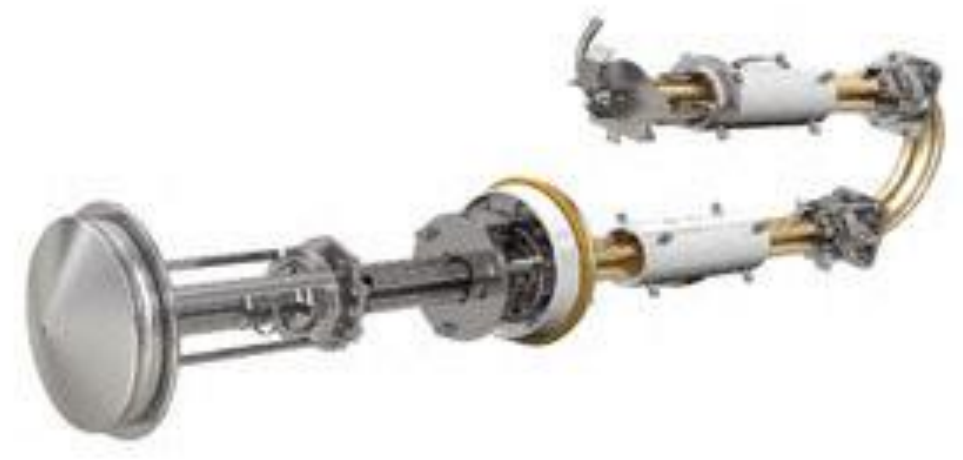

Figure 37: Picture of state-of-the-art QQQ mass spectrometer.

\subsection{Nitroaromatic Explosives}

There are several ways in which explosive compounds can accumulate in environmental waters. For example, bodies of water close to sites of former ammunition plants are likely to have explosive compounds in them [1]. Also, trace levels of contaminants can result indirectly from military exercises and lead 
to unexploded ordinances being released into oceans $[2,85,86]$. These contaminants in environmental waters can be hazardous to wildlife in these areas, and ultimately to humans [87]. Exposure to nitroaromatic explosives is dangerous even at trace level concentrations. For instance, the US EPA has set the acceptable limit for repeated exposure to TNT in drinking water at $2 \mathrm{ng} / \mathrm{mL}$ [5]. Ten nitroaromatic explosive compounds are included in this report, as stated in Table 1:

Table 1: List of the ten nitroaromatic explosive compounds included in this report, including their structure with the corresponding octanol-water partition coefficient (log Kow) available, it is given.

\begin{tabular}{|c|c|c|c|}
\hline $\begin{array}{c}\text { Compound: log } \\
\text { Kow }^{\circ}\end{array}$ & Structure & $\begin{array}{l}\text { Compound: } \\
\text { log Kow }\end{array}$ & Structure \\
\hline $\begin{array}{c}1,3,5- \\
\text { Trinitrobenzene } \\
\text { (TNB): } 1.2[88]\end{array}$ & & $\begin{array}{c}2,6- \\
\text { Dinitrotoluene } \\
(2,6-D N T): 2.1 \\
{[89]}\end{array}$ & \\
\hline $\begin{array}{c}1,3- \\
\text { Dinitrobenzene } \\
(1,3-D N B): 1.5 \\
{[90]}\end{array}$ & & $\begin{array}{c}\text { 2-Nitrotoluene } \\
\text { (2-NT): } 2.3 \\
\text { [91] }\end{array}$ & \\
\hline $\begin{array}{l}\text { Nitrobenzene } \\
\text { (NB): } 1.9 \text { [92] }\end{array}$ & & $\begin{array}{c}\text { 4-Nitrotoluene } \\
\text { (4-NT): } 2.4 \\
\text { [93] }\end{array}$ & \\
\hline $\begin{array}{l}2,4,6- \\
\text { Trinitrotoluene } \\
\text { (TNT): } 1.7 \text { [94] }\end{array}$ & & $\begin{array}{c}\text { 3-Nitrotoluene } \\
\text { (3-NT): } 2.5 \\
\text { [95] }\end{array}$ & \\
\hline
\end{tabular}




\begin{tabular}{|c||c|}
\hline $\begin{array}{c}2,4,6- \\
\text { (Trinitrophenyl) } \\
\text { methylmitroamine } \\
\text { (tetryl): } 1.6[96]\end{array}$ & $\begin{array}{c}\text { 4-Amino-2,6- } \\
\text { dinitrotoluene } \\
\text { (4-am-2,6- } \\
\text { DNT) }\end{array}$ \\
\hline
\end{tabular}

An example of a body of water that has been contaminated by explosive compounds can be found in Switzerland, where it is estimated that approximately 4,880 combined tons of ammunition waste were deposited in Lakes Thun and Brienz between the years 1920 and 1967 [97]. This ammunition waste includes hundreds of tons of nitroaromatic explosives, the most abundant ones being trinitrotoluene and 1,3-dinitrobenzene [97]. Harmful effects of this may have already been seen, as local fishermen have noticed anomalies in the reproductive organs of white fish from Lake Thun since the year 2000 [97]. The specific deformities seen in the white fish caught at Lake Thun had never before been described in the literature $[97,98]$.

\subsection{BTEX}

Benzene, toluene, ethylbenzene, ortho-, meta-, and paraxylene constitute an important class of environmental pollutant compounds. The data presented herein includes all those compounds except m-xylene (Table 2): 
Table 2: List of the 5 BTEX compounds included in this report, in addition to the structure and octanol-water partition coefficient (log Kow) of each.

\begin{tabular}{|c|c|c|c|}
\hline $\begin{array}{c}\text { Compound: log } \\
\mathrm{K}_{\text {ow }}\end{array}$ & Structure & $\begin{array}{l}\text { Compound: } \\
\text { log Kow }\end{array}$ & Structure \\
\hline $\begin{array}{c}\text { Benzene: } 2.1 \\
{[99]}\end{array}$ & & $\begin{array}{c}\text { o-Xylene: } 3.1 \\
\text { [100] }\end{array}$ & \\
\hline $\begin{array}{c}\text { Toluene: } 2.7 \\
\text { [101] }\end{array}$ & & $\begin{array}{c}\text { p-Xylene: } 3.2 \\
{[102]}\end{array}$ & \\
\hline $\begin{array}{c}\text { Ethylbenzene: } \\
3.2[103]\end{array}$ & & & \\
\hline
\end{tabular}

All BTEX compounds are carcinogenic, to the extent that in the United States the maximum air concentration of benzene is limited to $1 \mu \mathrm{g} / \mathrm{m}^{3}$ [104]. Not only are these compounds very toxic, there is a wide variety of entry points into the environment that in modern societies are impossible to eliminate: normal working processes of power plants [6], petroleum derivatives [105], automobile exhaust engines [7], and even domestic use of heaters and gas boilers [106,107]. The overwhelming number of common sources of BTEX compounds does not necessarily mean that the environment is or will be contaminated enough to be unfit for human life, but it certainly makes it imperative that regulatory methods 
be able to reliably quantify their presence in environmental water at ultra-trace levels of concentration.

\subsection{Polycyclic Aromatic Hydrocarbons}

There are $16 \mathrm{PAHs}$ that are considered priority pollutants by the US EPA because they are known carcinogens and pose a grave threat to the health of humans and ecosystems $[108,109]$. These compounds result from combustion processes, so their existence in the environment will become more prevalent over time, not less, and having the ability to accurately measure their concentrations in environmental waters and other samples is very important [109]. It is not only regulatory agencies in the United States that place great importance on this class of compounds; in fact, PAHs are recognized as the second most abundant type of environmental pollutant in China [110]. Considering that PAHs are priority pollutants because of their carcinogenic properties [111], it is quite worrisome that their existence is so prevalent and growing. PAHs have been found both in fresh and saline environmental waters $[108,111]$. The data presented here includes five of the $16 \mathrm{PAHs}$ that are priority pollutants (Table 3 ): 
Table 3: List of the 5 PAHs included in this report, including the structure and octanol-water partition coefficient (log Kow) of each.

\begin{tabular}{|c|c|}
\hline $\begin{array}{c}\text { Compound: log } \\
\text { Kow }\end{array}$ & $\begin{array}{c}\text { Compound: } \\
\text { log Kow }\end{array}$ \\
\hline $\begin{array}{c}\text { Naphthalene: } \\
3.3 \text { [112] }\end{array}$ \\
\hline $\begin{array}{c}\text { Anthracene: } \\
4.5[113]\end{array}$ \\
\hline $\begin{array}{c}\text { Fluorene: } 4.2 \\
\text { [114] }\end{array}$ \\
\hline $\begin{array}{c}\text { Phenanthrene: } \\
\text { 4.5 [116] }\end{array}$ \\
\hline
\end{tabular}

Fungal enzymes such as Phanerochaete chrysosporium, Pleurotus ostreatus, and Bjerkandera adusta have been found to be a promising option for the biodegradation of PAHs in environmental matrices [117], therefore the detection of ultra-trace concentrations of these compounds so that they can be neutralized before damaging humans or the ecosystem serves more of a purpose than simply warning about contaminated areas, it can lead to renewal of previous environmental resources.

\subsection{Substituted Phenols}

Phenol and derivatives of it, known collectively as substituted phenols, are very dangerous to humans because of their toxicity $[9,10]$. Compounds in this class bind to hemoglobin [9] and are known to be particularly harmful to the urinary, 
circulatory, respiratory and central nervous systems of human beings depending on the amount of exposure and how prolonged that exposure is [11]. In many cases the substitutions on phenols are methyl groups, which, in addition to the hydroxyl group and the aromatic ring gives them attributes very desirable for industrial use [9]. Wood processing plants, pharmaceutical labs and oil refineries are all enterprises that commonly use substituted phenols and as such their waste disposal practices should be closely monitored and inspected $[9,10]$. Degradation of soil can also produce methyl substituted phenols as by-products [9]. The data presented here include method development and validation for detection and quantitation of the substituted phenols in Table 4:

Table 4: List of the 5 substituted phenols included in this report, including the structure and octanol-water coefficient (log Kow) of each.

\begin{tabular}{|c|c|c|c|}
\hline $\begin{array}{c}\text { Compound: log } \\
\mathrm{K}_{\mathrm{ow}}\end{array}$ & Structure & $\begin{array}{c}\text { Compound: log } \\
\mathrm{K}_{\mathrm{ow}}\end{array}$ & Structure \\
\hline $\begin{array}{l}\text { 4-Chlorophenol } \\
(4-C P): 2.4[10]\end{array}$ & & $\begin{array}{c}2,4,6- \\
\text { Trichlorophenol } \\
\text { (TCP): } 3.7 \\
{[118]}\end{array}$ & \\
\hline $\begin{array}{c}3,5- \\
\text { Dimethylphenol } \\
(3,5-D M P): 2.4 \\
{[118]}\end{array}$ & & $\begin{array}{c}\text { 2,4-Diiso- } \\
\text { propylphenol } \\
\text { (2,4-DIPP): } \\
\text { N/A }\end{array}$ & \\
\hline $\begin{array}{c}2,6- \\
\text { Dichlorophenol } \\
(2,6-D C P): 2.7 \\
{[10]}\end{array}$ & & & \\
\hline
\end{tabular}




\subsection{Endocrine Disrupting Chemicals in Milk}

Milk and the plethora of products derived from it are a large component of the world's nutrition for humans and animals. The possibility of consuming milk contaminated with harmful contaminants such as Endocrine Disrupting Compounds (EDCs) is of major concern [119]. The risk of milk posing a threat to the consumer's health is never more substantial than when the consumer is a fetus (indirectly, from the mother) or an infant; not only is milk typically the only source of nutrition for infants, they are more susceptible to harmful effects from xenobiotics because of their relatively low body mass. The danger extends to adults because most humans consume milk and other dairy products past their childhood and are therefore at some level of risk throughout their lives. In response to these concerns, regulatory agencies have passed measures to safeguard the safety of consumption of dairy products. For example, the European Union (EU) imposed Maximum Residue Limits (MRLs) for veterinary substances administered to food-producing animals as part of Council Regulation 2377/90/EEC and has also set forth guidelines for analytical method performance in the determination of organic residues in animal products [120,121]. Said guidelines can be found in the 657/2002/EC EU Commission Decision.

Environmental pollution of EDCs due to the disposal of certain products (e.g. birth control medication) [122] and their presence in plastics (BPA) $[123,124]$ places milk-producing cows and women that have aspirations of being pregnant, are pregnant, or breastfeeding at risk. In regards to BPA alone, 5.5 million metric tons were produced in 2011 [125] and this is a compound to which humans can 
be exposed not only through their diet but also dust inhalation and dermal absorption [124,126]. Research on the consequences of women's exposure to EDCs has yielded the following alarming findings that suggest that having the ability to quantify EDCs in all matrices is of utmost importance: abnormalities that are collectively referred to as Ovarian Dysgenesis Syndrome (ODS) including abnormal ovarian and Mullerian duct development and reproductive tract cancers; abnormal placentation and placental function due to inhibition of trophoblast differentiation caused by maternal exposure to EDCs; disturbance of the neuroendocrine systems within the hypothalamus and pituitary gland causing disruption of gonadal function; and in the case of pregnant women the fetus is likely exposed to EDCs if the mother is, placing them both at great risk [127]. Additionally, exposure of men to EDCs has already been shown to decrease sperm count, cause cancer (testicles, breasts, prostate) [128], and lead to Testicular Dysgenesis Syndrome (TDS) which is an umbrella term that includes hypospadias (urogenital tract malformation), cryptorchidism (testis maldescent) and testicular cancer [129].

The 6 EDCs in Table 5 are one of the possible threats to the safety of milk as a vital nutrition source [128] and are considered emerging contaminants because of the recent upward trend of their concentration in the environment [130] which is currently estimated to be up to several thousand $\mathrm{ng} / \mathrm{L}$ in some places [12]. 
Table 5: Structure, source of prduction and octanol-water partition coefficient (log Kow) of each endocrine disruptor included in this study.

\begin{tabular}{|c|c|c|c|}
\hline Compound & Structure & $\begin{array}{l}\text { Drug Class } \\
\text { or Source }\end{array}$ & Log Kow \\
\hline $\begin{array}{l}\text { Bisphenol A } \\
\text { (BPA) }\end{array}$ & & $\begin{array}{l}\text { Originally synthetic } \\
\text { hormone, present in } \\
\text { plastics and other } \\
\text { products }\end{array}$ & $\begin{array}{l}3.7 \\
{[118]}\end{array}$ \\
\hline $\begin{array}{l}\text { Estradiol } \\
\text { (E2) }\end{array}$ & & $\begin{array}{c}\text { Natural } \\
\text { Estrogen } \\
\text { Derivative }\end{array}$ & $\begin{array}{l}4.0 \\
{[118]}\end{array}$ \\
\hline $\begin{array}{l}\text { Estrone } \\
\text { (E1) }\end{array}$ & & $\begin{array}{c}\text { Natural } \\
\text { Estrogen } \\
\text { Derivative }\end{array}$ & $\begin{array}{l}3.1 \\
{[118]}\end{array}$ \\
\hline $\begin{array}{c}\text { 17a-Ethinyl } \\
\text { Estradiol (EE2) }\end{array}$ & & $\begin{array}{l}\text { Synthetic } \\
\text { Estradiol } \\
\text { Derivative }\end{array}$ & $\begin{array}{l}3.7 \\
{[118]}\end{array}$ \\
\hline $\begin{array}{l}\text { Diethylstilbestr } \\
\text { ol (DES) }\end{array}$ & & $\begin{array}{l}\text { Synthetic } \\
\text { Estrogen } \\
\text { Derivative }\end{array}$ & $\begin{array}{l}5.1 \\
{[118]}\end{array}$ \\
\hline $\begin{array}{l}\text { Hexestrol } \\
\text { (HEX) }\end{array}$ & & $\begin{array}{l}\text { Synthetic } \\
\text { Estrogen } \\
\text { Derivative }\end{array}$ & $\begin{array}{l}4.40 \\
{[131]}\end{array}$ \\
\hline
\end{tabular}

\section{OBJECTIVES AND SIGNIFICANCE OF STUDY}

There is no doubt that environmental and biological analyses are very important aspects of analytical chemistry. In fact, analysis of environmental and biological 
samples is very important within the vast branch of chemistry that is analytical chemistry. Globalization is technically interaction and integration among peoples, cultures, and societies throughout the world. This is a trend that started millennia ago, but has picked up very rapidly in the last three decades. Mostly driven by business, where a great example from the Middle Ages is the utilization of the Silk Road that connected China to Europe, globalization is something that the world must continue to adapt to efficiently. Transportation technologies, developing at an unprecedented and amazing rate, have made it so that someone can travel thousands of miles for lunch and be home for dinner. Comfortably traveling safely from one side of the world to another is so common that a person working in the United States below the middle class can manage to do it and no one would be surprised. These developments are very popular for many obvious reasons, both in the world of business and pleasure as people often travel for vacation to places that not so long ago were out of reach.

However, this also means that certain dangers are spread quickly throughout the world and someone could be exposed to certain toxins from faraway places. For those who travel, they consume water, vegetables, milk and other foods wherever they go and depend on those items being safe to consume. Even people who don't travel often are at risk of being exposed to harmful substances from other places because groceries can be imported from other places.

Nitroaromatic explosive compounds are a concern because of their hazardous nature to wildlife and humans even at concentrations in the range of low $\mathrm{ng} / \mathrm{mL}$; 
BTEX compounds and PAHs are known carcinogens that are constantly produced throughout the world; substituted phenols are toxic to the urinary, circulatory, respiratory and central nervous systems of human beings; and EDCs cause serious irregularities to the reproductive systems of both men and women, not to mention testicular, breast and prostate cancer. All these compounds are important environmental forensics and as such pose a toxicity risk to wildlife and the water and food supply of humans. EDCs are also of great concern in milk.

The best way to combat this problem is to prevent contaminated water and food supplies and these prevention efforts start with identifying which areas are contaminated. That is why the invention and development of FPSE is so important. This report conveys the complete picture of the effectiveness of FPSE by explaining the theory behind it and then presenting data to support the claim that it is superior for direct immersion analysis to any commercially available technology, thereby presenting a paradigm shift in the hierarchy of available methodologies for sample preparation of complex environmental and biological samples. This significance of this research has far-reaching implications because it clearly has many other applications, including safe storage of analytes at ambient conditions, for which irrefutable evidence is also included.

\section{MATERIALS AND INSTRUMENTATION}

\subsection{Materials}

The materials and chemicals that were used to make the FPSE media were the following: unbleached Muslin cellulose and polyester fabric substrates purchased 
from Jo-Ann Fabric in Miami, FL, USA; methyltrimethoxysilane (MTMS), trifluoroacetic acid (TFA), acetone, PTHF, polydimethyl siloxane, dichloromethane from Sigma-Aldrich located in St. Louis, MO, USA; polyethylene glycol from Alfa Aesar (Ward Hill, MA, USA); polydimethyldiphenyl siloxane from Gelest (Morrisville, PA, USA); and sodium hydroxide and hydrochloric acid from Thermofisher Scientific (Waltham, MA, USA).

The substituted phenols used: 4-chlorophenol ( $\geq 99 \%)$, 3,5-dimethylphenol (98\%), 2,6-dichlorophenol (99\%), 2,4,6-trichlorophenol (98\%), and 2,4-diisopropyl phenol (98\%) and all six EDCs: estradiol, hexestrol, estrone, 17a-ethinyl estradiol, diethylstilbestrol, and BPA were also purchased from Sigma-Aldrich. Also from Sigma-Aldrich are NB, 2,6-DNT, 4-NT, 3-NT, and 2-NT; 1,3-DNB is Acros Organics (Geel, Belgium), a division of Thermofisher; the rest of the nitroaromatic explosives (TNT, tetryl, TNB, 4-amino-2,6-DNT) are from Accustandard (New Haven, CT, USA). Regarding the BTEX compounds: toluene is from Fischer Scientific (Fair Lawn, NJ, USA); benzene, ethylbenzene, o- and p- xylene from Sigma-Aldrich. Of the 5 PAHs, anthracene is from Supelco (Bellefonte, PA, USA) which is a division of Sigma-Aldrich; naphthalene, fluorene, floranthene, and phenanthrene are from Sigma-Aldrich directly. The HPLC-grade solvents used (water, methanol and acetonitrile) are from the Fisher Chemical brand which is a part of Thermofisher Scientific. 


\subsection{Analytical Instrumentation}

The method development and most of the validation was performed on an Agilent 1100 series HPLC-UV system (Agilent Technologies, Santa Clara, CA, USA) equipped with G1311A quaternary pump, G1322A vacuum degasser, G1313A ALS auto sampler, tray holder (capacity to hold 100 vials), G1316A temperature controlled column compartment, G1314A variable wavelength detector (UV-vis wavelength range). Two HPLC columns were used: a Zorbax Extend-C18 purchased from Agilent, $150 \times 5$ mm, $4.6 \mu \mathrm{m}$ particle size, Agilent Technologies, Santa Clara, CA, USA; and an Ascentis RP Amide, $150 \times 5$ mm, 4.6 $\mu \mathrm{m}$ particle size, from Sigma Aldrich. The centrifuge used to remove any large particles after back extraction is an Eppendorf Centrifuge $5415 \mathrm{R}$. The microscopic images were generated with a JEOL JSM 5900LV SEM. The Fourier Transform Infra-Red (FT-IR) spectra were obtained with a PerkinEImer Spectrum 100 FT-IR Spectrometer.

The HPLC-MS/MS analysis was conducted on a Thermo Finnigan Surveyor Plus HPLC system coupled to an Applied Biosystems Sciex QTRAP 5500 TripleQuadrupole mass spectrometer, equipped with a Turbo V ${ }^{\mathrm{TM}} \mathrm{ESI}$ ionization source. The same column mentioned above (Zorbax Extend-C18 purchased from Agilent, $150 \times 5 \mathrm{~mm}, 4.6 \mu \mathrm{m}$ particle size) was used on this instrument. 


\section{METHODOLOGY, RESULTS AND DISCUSSION}

\subsection{Preparation of FPSE Media}

When the research presented here started, FPSE was a completely untested, unknown invention. As such, FPSE media were synthesized for the first time in house, at the Furton Research Laboratory at FIU, and still are. After much research optimizing the composition of the sol-gel materials that were ultimately used for FPSE media, the following major steps led to the finished product.

\subsubsection{Activation of Substrates}

Fabric phase sorptive extraction media are typically cut to $5 \mathrm{~cm}^{2}(2.5 \mathrm{~cm} \times 2 \mathrm{~cm})$ pieces for use, as was the case for the ones used for the following research. This can be changed according to different needs or preferences. This option is available because when the synthesis is done, much larger segments are typically made $\left(100 \mathrm{~cm}^{2}\right)$. The fabric is first washed with deionized water, then submerged into a strong basic solution $(0.1 \mathrm{M} \mathrm{NaOH})$ for one hour. External stimulation is applied here in the form of sonication. After this step is completed the $\mathrm{NaOH}$ is removed and any remaining amounts of it are removed by washing again with deionized water. At this point the fabric is dried in an in-house chamber that utilizes a continuous supply of helium gas and controlled heat $\left(50^{\circ} \mathrm{C}\right)$. This can be left overnight if it is convenient. The fabric is now prepared for sol-gel coating, but this does not have to happen immediately and in fact can be postponed for as long as necessary by keeping it in a clean, air-tight container. 


\subsubsection{Preparation of Sol Solutions}

The general procedure for the synthesis of the sol solutions is an adaptation of a procedure first described by Malik et al. in 1997, when it was used to make capillary GC columns [132]. This general process has been described several times since in the literature $[10,16,56]$ and has been used consistently for the synthesis of sol-gel materials that serve as FPSE media sorbents. The sol-gel process, as described previously, consists of hydrolysis of an inorganic precursor followed by poly-condensation to create a three-dimensional network. For the sorbents used to make the FPSE media used to gather the data presented here the following ingredients and amount were used:

- $10 \mathrm{~mL}$ methyltrimethoxysilane

- $20 \mathrm{~mL}$ methylene chloride

- $4 \mathrm{~mL}$ trifluoroacetic acid, $95 \%$

- $10 \mathrm{~g}$ of the organic polymer

- PEG, PTHF, PDMS, or PDMDPS

This mixture is vortexed for 3 minutes to ensure that it is homogeneous and centrifuged for 5 more minutes. The methylene chloride serves as the organic solvent, while the TFA is the catalyst for the hydrolysis of the inorganic precursor, MTMS. Centrifugation results in the formation of a clear supernatant, which is the sol solution. This supernatant is transferred to a clean amber bottle and eventually conditioning of this sol solution results in poly-condensation and coating of the pretreated fabric. 


\subsubsection{Creation of Sol-gel Coatings on Substrates}

The clean, previously treated fabric is submerged in the reaction bottle with the sol solution. This is when the three-dimensional network forms. The coating process takes 4 hours. After this is done, the now coated fabrics are removed from the sol solution and placed in a conditioning device made by adapting a GC oven. The conditioning process takes 24 hours, utilizing heat $\left(50^{\circ} \mathrm{C}\right)$ and a continuous flow of helium gas, not unlike the drying process after washing with deionized water. After the 24 hour period the coated fabrics are washed first with methylene chloride and then with methanol to remove any of the sol-gel material that may be on it but not bonded to it. After these two washing periods the FPSE media are dried one last time at $50^{\circ} \mathrm{C}$ for 1 hour. The process of synthesis is now complete and the $100 \mathrm{~cm}^{2}$ FPSE medium can be cut to the desired shape and size.

\subsection{Characterization of FPSE Media}

A major part of this research was characterizing FPSE media with SEM imaging and FT-IR spectroscopy.

\subsubsection{Scanning Electron Microscopy}

SEM imaging is a great tool for characterization of samples. These instruments do not use light. Instead, in a vacuum, bombardment of the sample with a focused electron beam produces emittance of other electrons. These other electrons are detected and transformed into an image by the corresponding software. Scanning electron microscopes consist of an electron source, lenses, 
scanning coil, sample chamber, and detectors. The electron source produces the electron beam; the lenses focus the electron beam from the source to the sample; the scanning coil allows scanning of the sample surface by moving the focused electron beam; the sample chamber is where the sample is placed for analysis, it can include useful features such as tilt and rotation and optical cameras; the SEM detectors collect the secondary electrons (SE) and backscattered electrons (BSE) that are used to form the image [133]. Fig. 38 shows a diagram of a SEM [134].

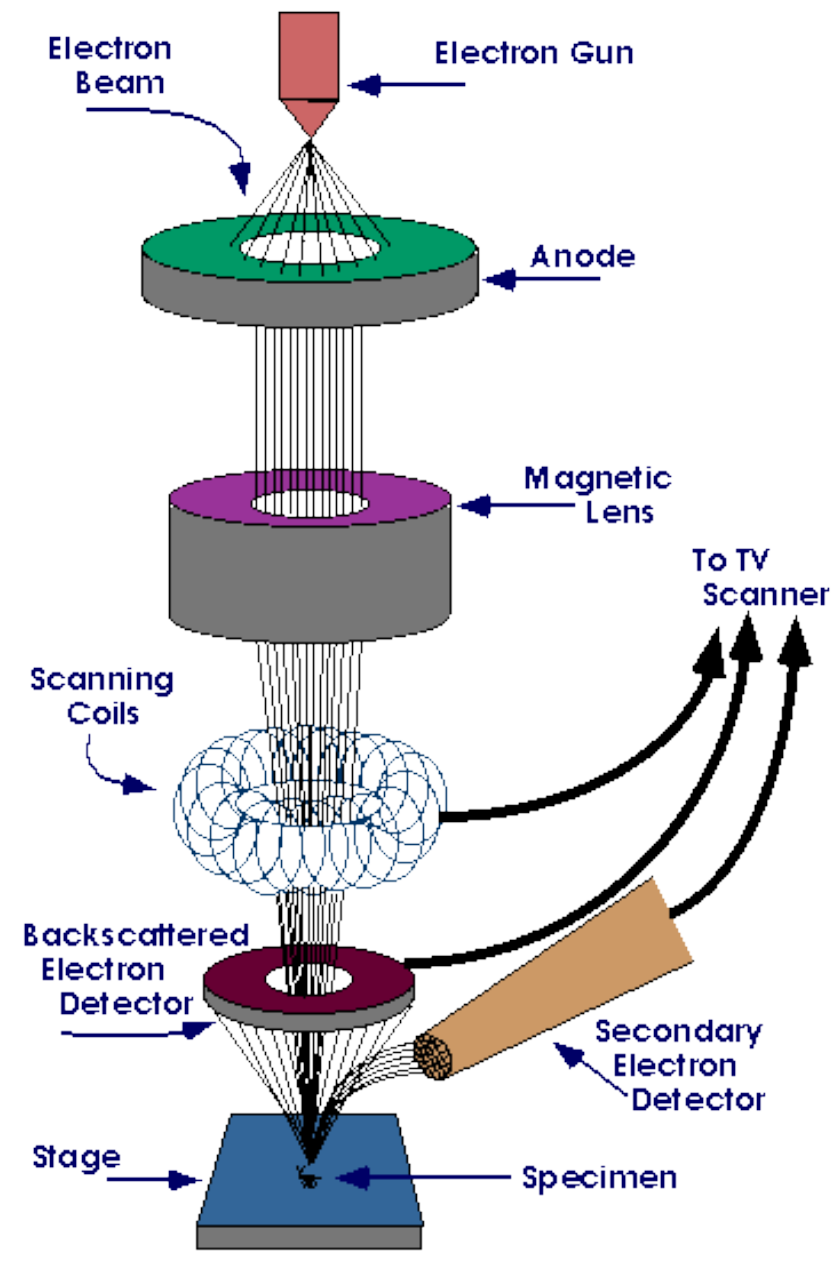

Figure 38: Labeled diagram of a scanning electron microscope. 
SEs and BSEs are both produced in the interaction between electrons from the electron beam and the sample, but are different and so are the purposes they serve (see Fig. 39) [133]. SEs tend to be of relatively low energy, ejected from kshells of atoms in the sample (the closest shell to the nucleus), and generally detected by scintillation, which produces a current that is amplified by a photomultiplier [133]. BSEs are higher in energy than secondary electrons and can give compositional information of the sample because these are produced from by atoms of high atomic number [133]. SEM imaging is considered a superior technology to light microscopes because it offers of a larger depth of field, so more of a sample can be looked at [134].

\section{Electron beam}

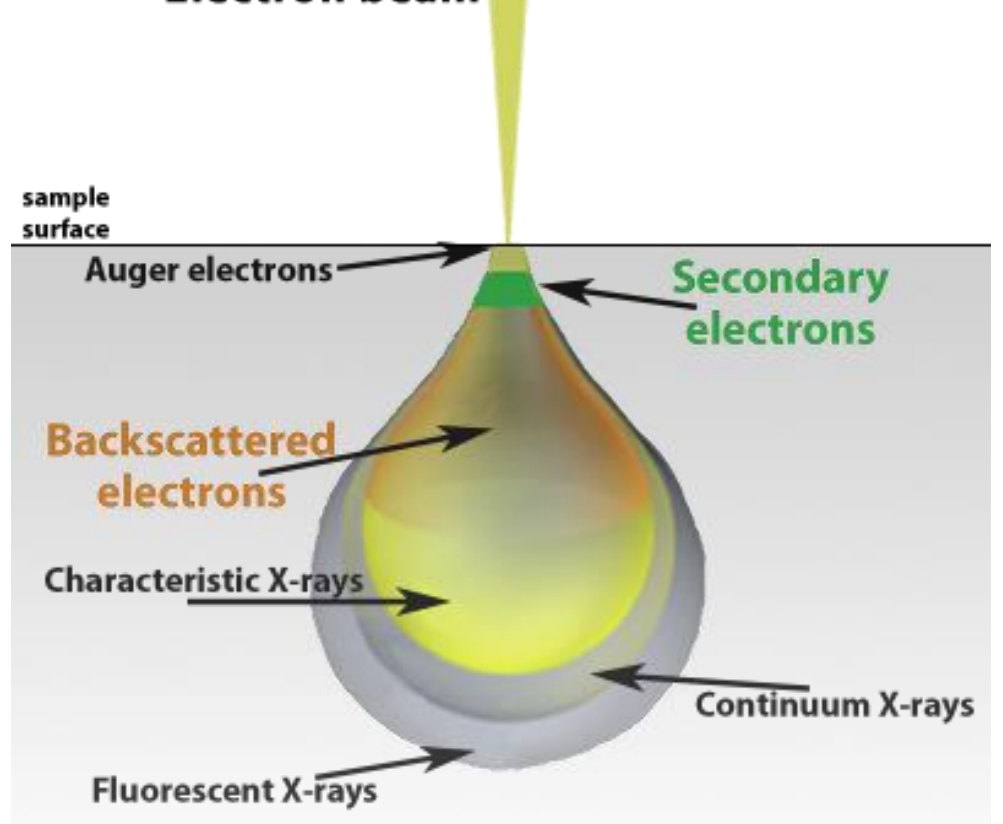

Figure 39: Labeled schematic of the interaction between electrons from the electron beam and the sample in SEM. 
The following sets of pictures are output SEM images gathered to elucidate the differences in the surfaces of uncoated cellulose and polyester, and corresponding FPSE media which have sol-gel sorbent bonded on the surface of the substrate (Figures $40-45$ ):
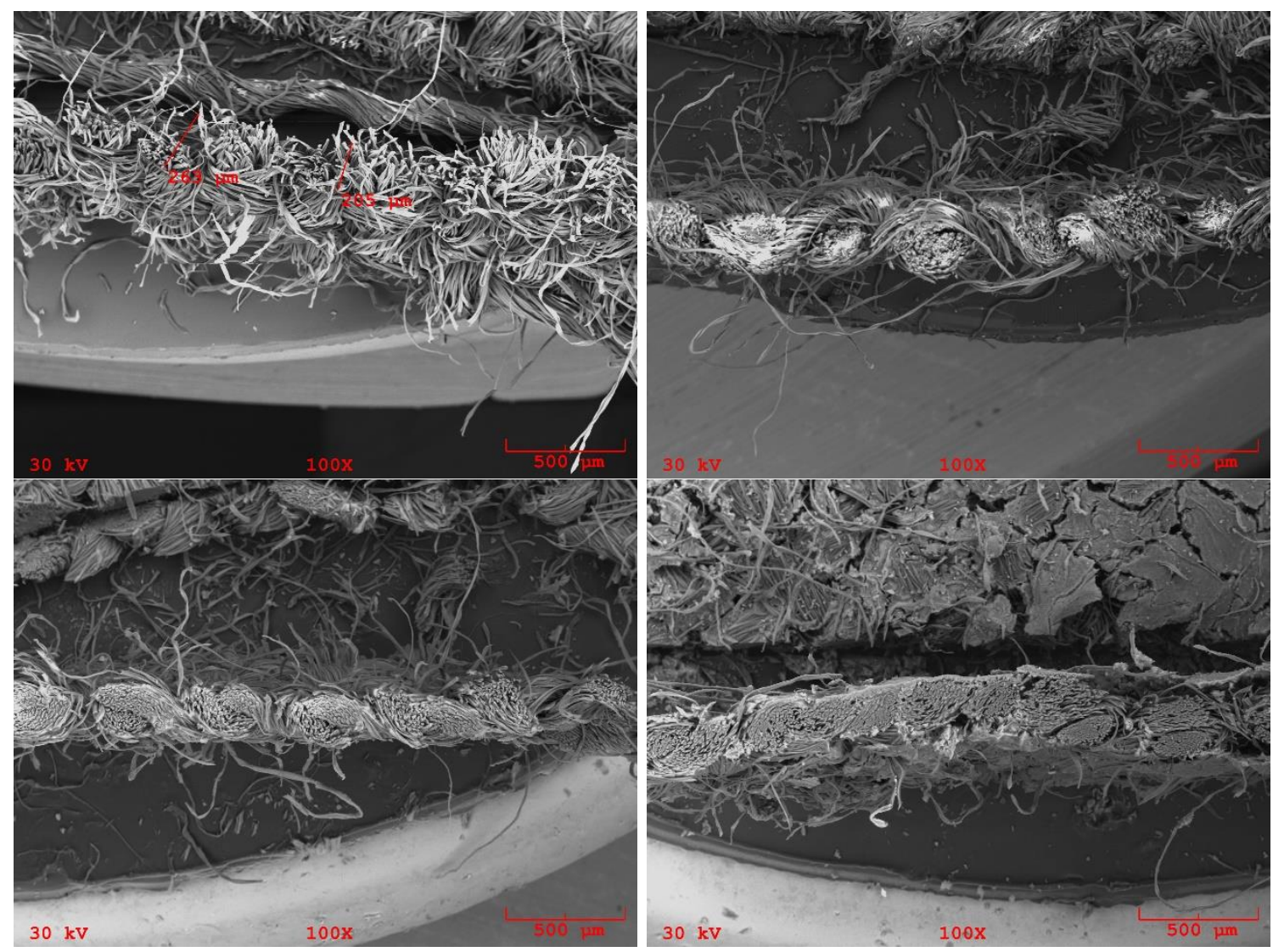

Figure 40: Cross-sectional 100x magnified BSE generated SEM image of uncoated cellulose fabric (top left); sol-gel MTMS coated cellulose fabric (top right); PTHF FPSE media on cellulose (bottom left); PEG FPSE media on cellulose (bottom right). 

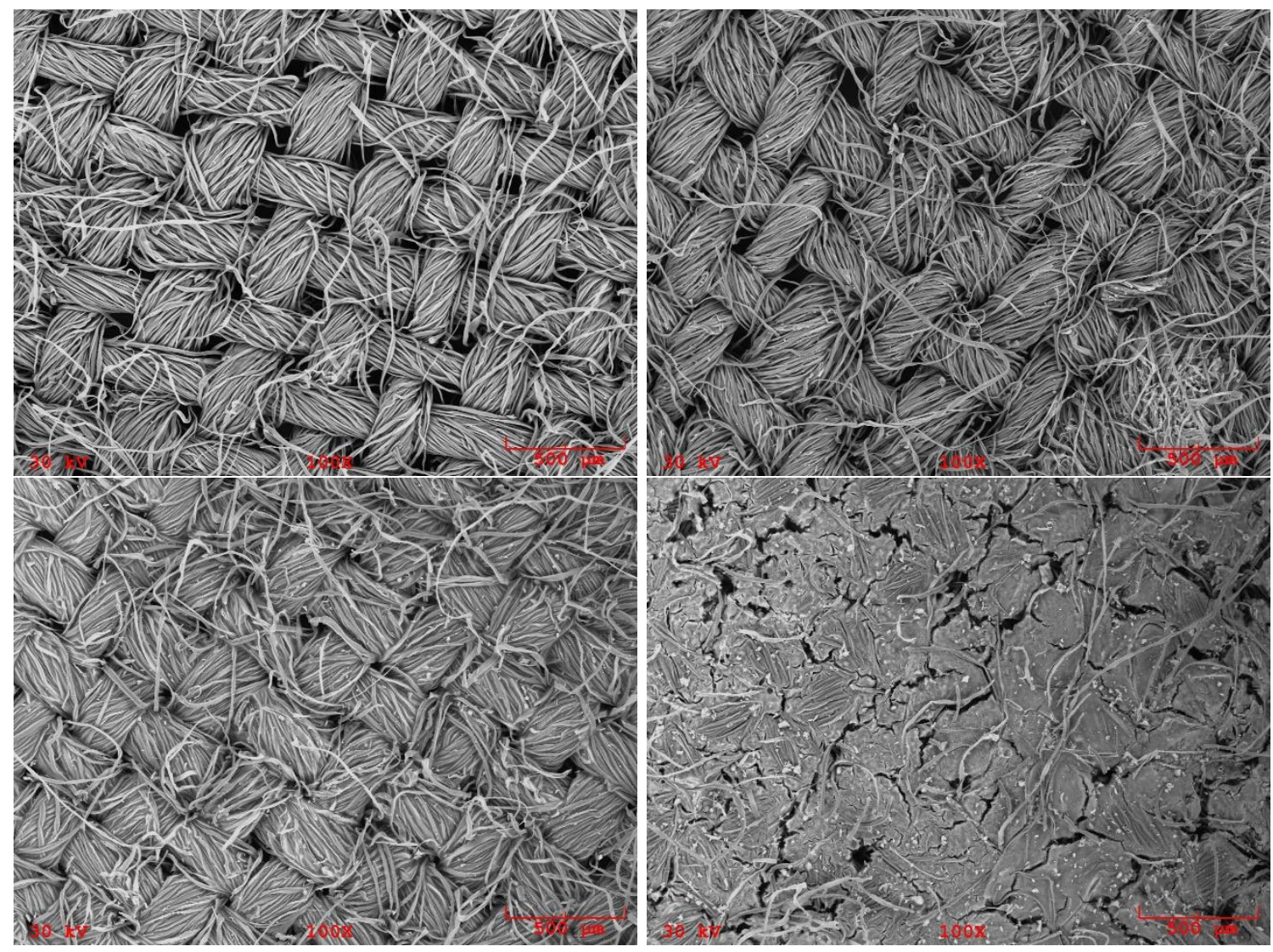

Figure 41: 100x magnified BSE generated SEM surface view of uncoated cellulose fabric (top left); sol-gel MTMS coated cellulose fabric (top right); PTHF FPSE media on cellulose (bottom left); PEG FPSE media on cellulose (bottom right). 

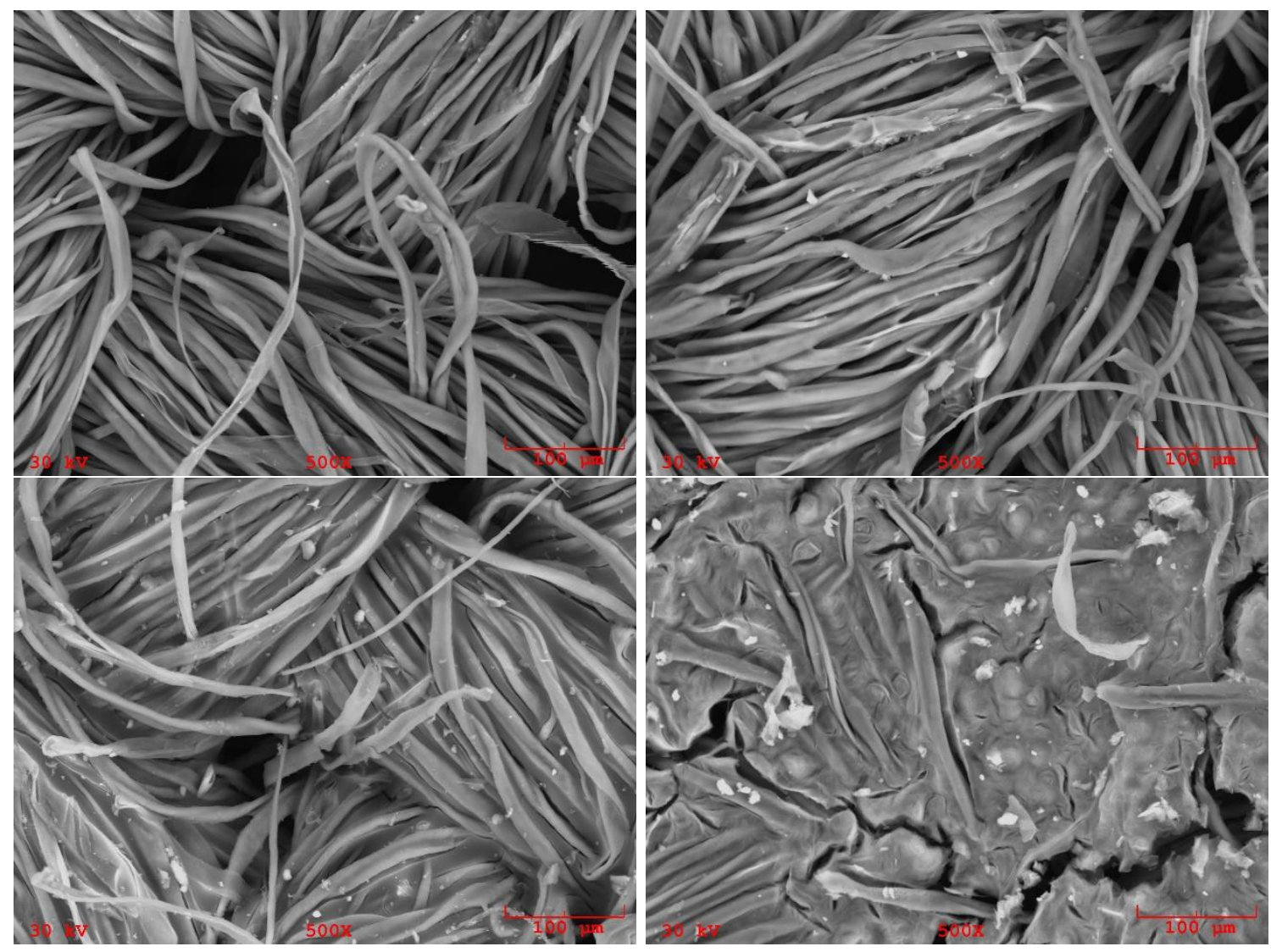

Figure 42: 500x magnified BSE generated SEM surface view of uncoated cellulose fabric (top left); sol-gel MTMS coated cellulose fabric (top right); PTHF FPSE media on cellulose (bottom left); PEG FPSE media on cellulose (bottom right).

Figures $40-42$ show very clear images of untreated cellulose fabrics, Fig. 42 is at 500x magnification and even shows individual fibrils. Each figure, however, shows four different images that ultimately show a progression from fabric without coating (top left in each picture), to a very thin and barely noticeable MTMS coating (top right), to a much more noticeable coating (FPSE-PTHF media) (bottom left), and ultimately to a relatively thick coating (FPSE-PEG media) (bottom right). This is all as expected. The top left images in Figs. $40-42$ all correspond to uncoated cellulose and the top right images all correspond to cellulose fabric coated with a sol-gel material made with MTMS but no organic 
polymer, which explains the very thin nature of the coating present. The pictures on the bottom left and right images in each of these figures are FPSE media, and as such have a sol-gel sorbent made with MTMS but also an organic polymer, resulting in the increased coating thickness. The sorbent on PEG FPSE media (bottom right image in Figs. $40-42$ ) is appreciably more voluminous than that of PTHF FPSE media (bottom left image in Figs. 40 - 42) because the PEG polymer molecules are much longer than the PTHF molecules.

Comparisons were also made of uncoated polyester fabric substrate vs FPSEPDMDPS media on polyester. The images are shown in Figs. $43-45$ :
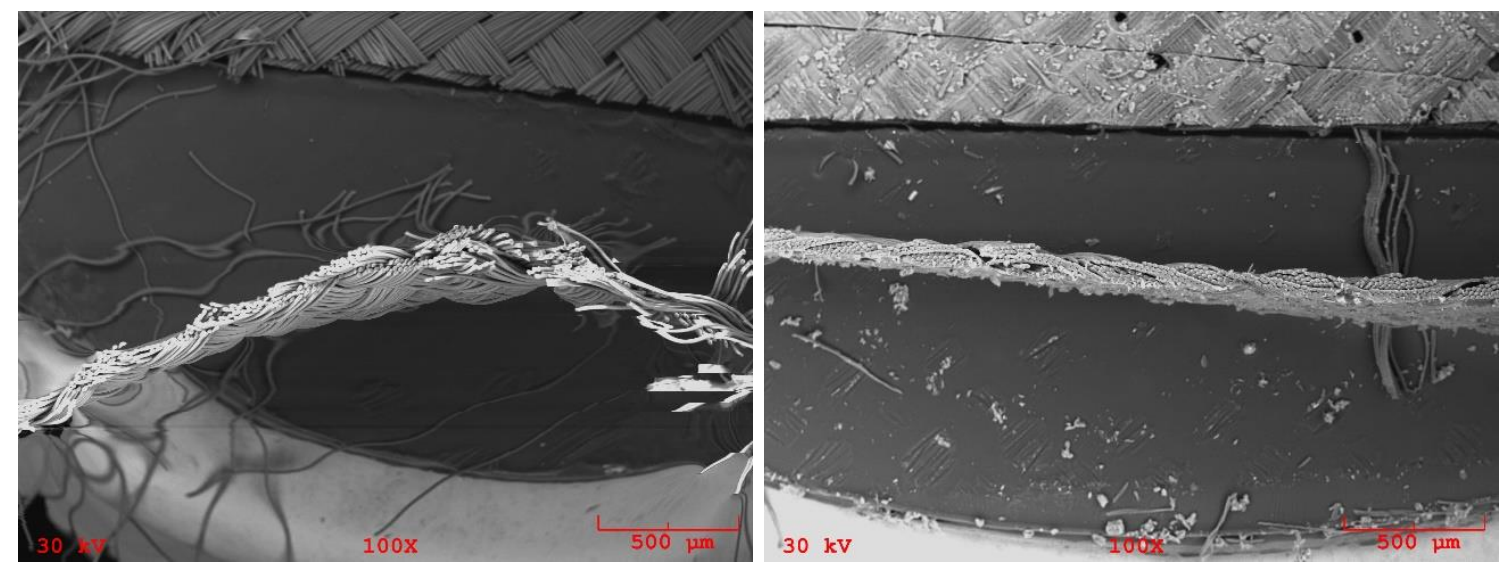

Figure 43: Cross-sectional 100x magnified BSE generated SEM image of uncoated polyester fabric (left); PDMDPS FPSE media (right). 

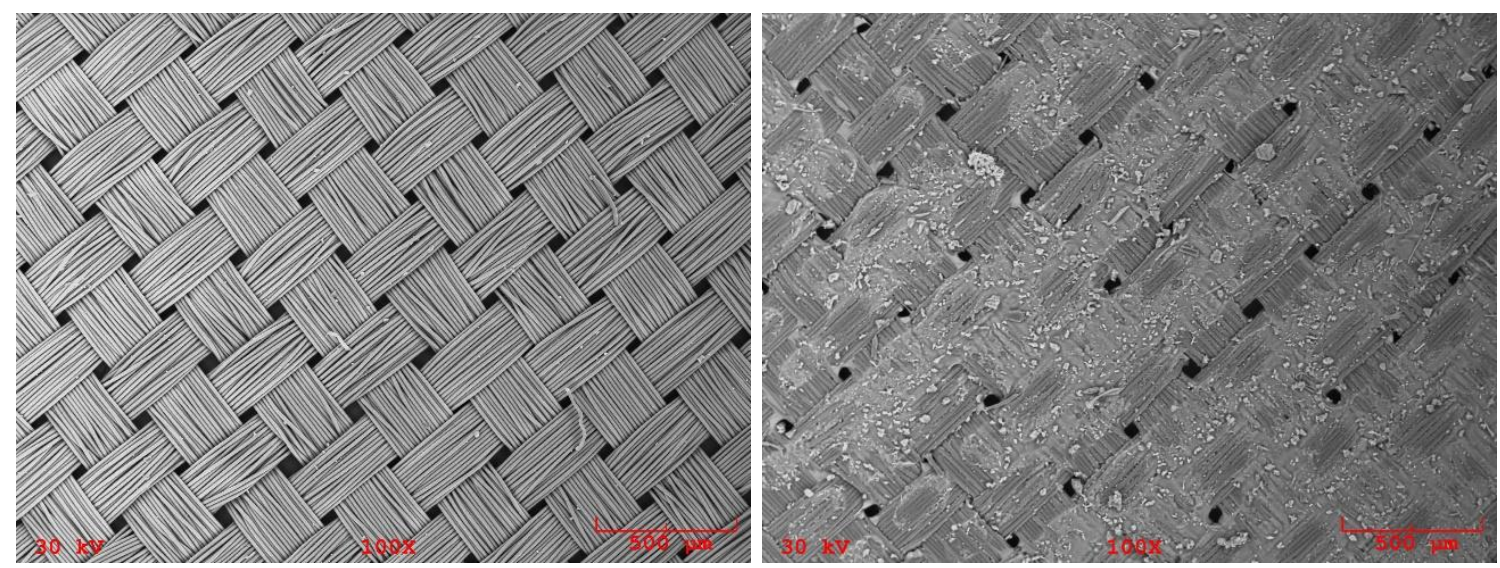

Figure 44: 100x magnified BSE generated SEM surface view of uncoated polyester fabric (left); PDMDPS FPSE media (right).
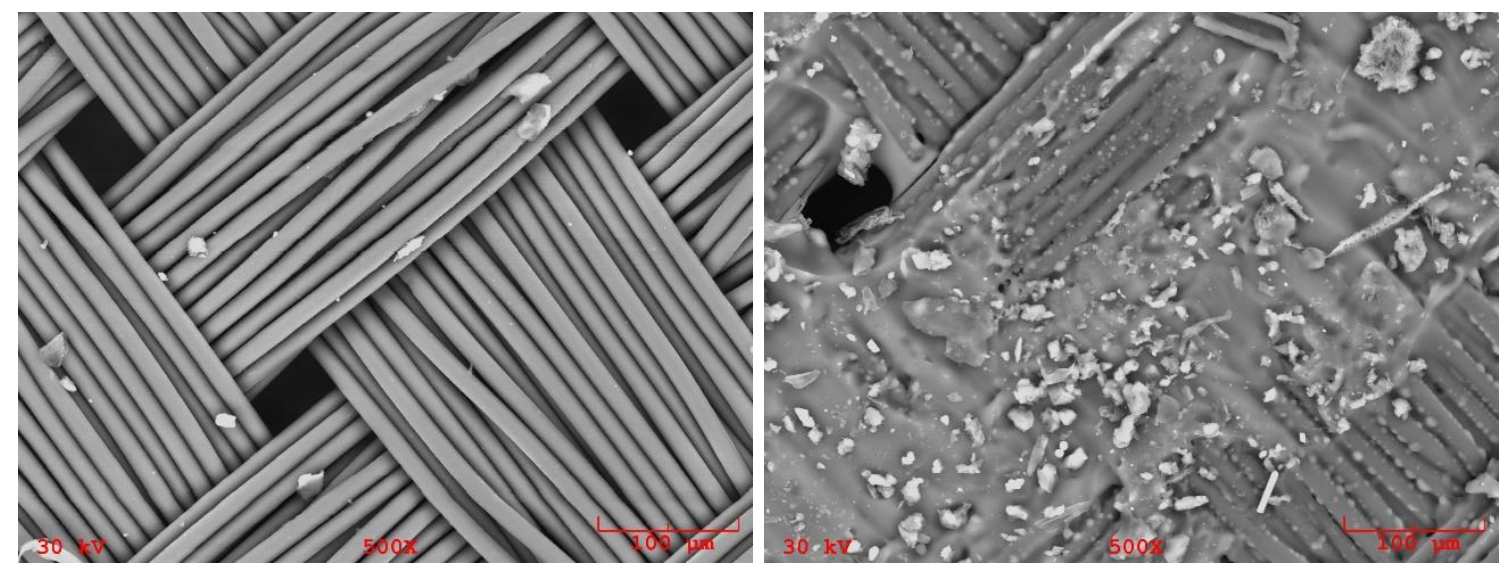

Figure 45: 500x magnified BSE generated SEM surface view of uncoated polyester fabric (left); PDMDPS FPSE media (right).

Figures $43-45$ tell a very similar story to that of Figures $40-42$. The first images in each figure (left side) show unemcumbered views of polyester fabrics.

The fabric looks very clean with some particles on them but no noticeable coating. The second images in each figure show the corresponding view of PDMDPS FPSE media on polyester. In all three figures it is clear that the PDMDPS sorbent is indeed bonded to the surface of the polyester substrate. 


\subsubsection{FT-IR Spectroscopy}

SEM imaging proved very effective for characterization of FPSE media, but (Fourier Transform Infra-Red Spectroscopy) FT-IR spectroscopy is effective in a different manner and therefore it was used as well. Fourier transform, which has been used on practically all IR spectrometers since computers became relatively practical and accessible some 30 years ago [135], is essentially the application of a mathematical function that converts the raw data from the infra-red spectrometer to a format that is much more convenient to interpret. Specifically, the signal is changed from being a function of time to a function of frequency [135].

The IR part of the instrument is where the chemistry comes in, it is a study of the interaction of light waves in the IR region of the electromagnetic spectrum with molecules in the sample. IR spectra are most commonly obtained in terms of wavenumbers, which are inverse of wavelengths, and the range analyzed is 600 $-4000 \mathrm{~cm}^{-1}$. Molecules have characteristic IR spectra because each functional group produces a recognizable absorption pattern [135]. Per the bond strength, mass of the atoms and the surrounding of the molecule, vibrations and stretching of bonds that change dipole moment create specific frequencies that absorb IR waves [135]. Once the spectrum is obtained, it can either be compared to the spectrum of a known molecule or it can be analyzed by identifying absorption patterns in the spectrum that correspond to certain functional groups. Figures 46 - 48 show results of FT-IR comparisons of FPSE media, the organic polymer in the corresponding sol-gel sorbent, and the relevant substrate. Figures 46 and 47 
include an FT-IR spectrum of uncoated cellulose, which exhibits a characteristic broad absorption band between $3100 \mathrm{~cm}^{-1}$ to $3400 \mathrm{~cm}^{-1}$ that corresponds to $\mathrm{OH}$; additional absorption between $2894 \mathrm{~cm}^{-1}$ and $1308 \mathrm{~cm}^{1}$ and at $1024 \mathrm{~cm}^{-1}$ correspond to $\mathrm{C}-\mathrm{H}$ vibration and $\mathrm{C}-\mathrm{O}$ bending, respectively $[10,56]$. The second spectrum in Fig. 46 does not show any absorption specific to PTHF but there is still evidence of the sol-gel sorbent being bonded on the fabric because the characteristic absorption peaks of the uncoated cellulose are reduced [56]. The broad band corresponding to $\mathrm{OH}$ where the bonding of the sorbent takes place is an example of a reduced response. Additionally, the peak at $1023 \mathrm{~cm}^{-1}$ on the spectrum corresponding to the PTHF FPSE media is evidence of C-O-C bridge bonds, $[10,16,56]$ a product of bonding of the sorbent to $\mathrm{OH}$ groups on the surface of the substrate. 

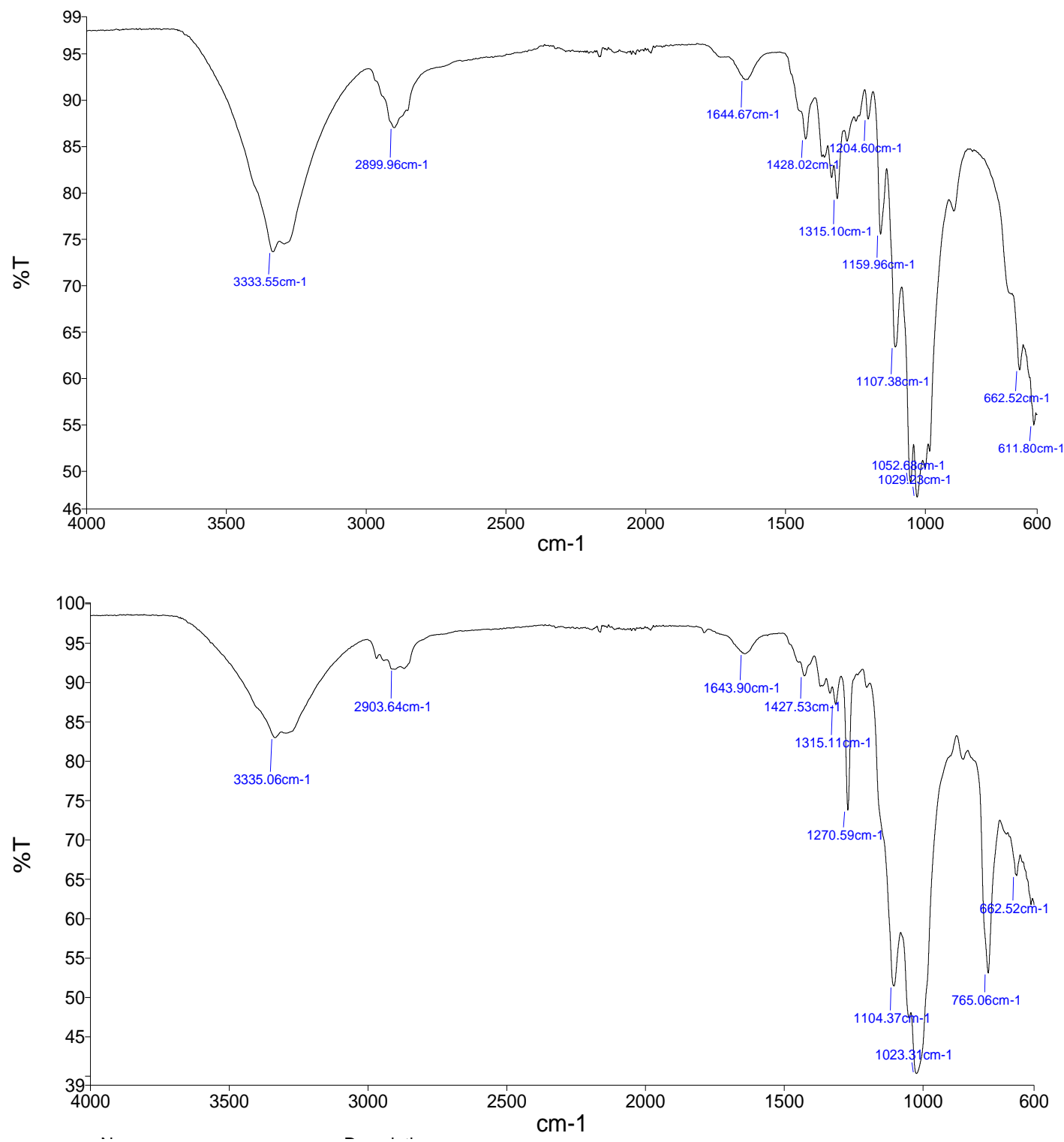

Figure 46: The top FT-IR spectrum is of uncoated cellulose fabric, the one at the bottom is of PTHF FPSE media on cellulose. 

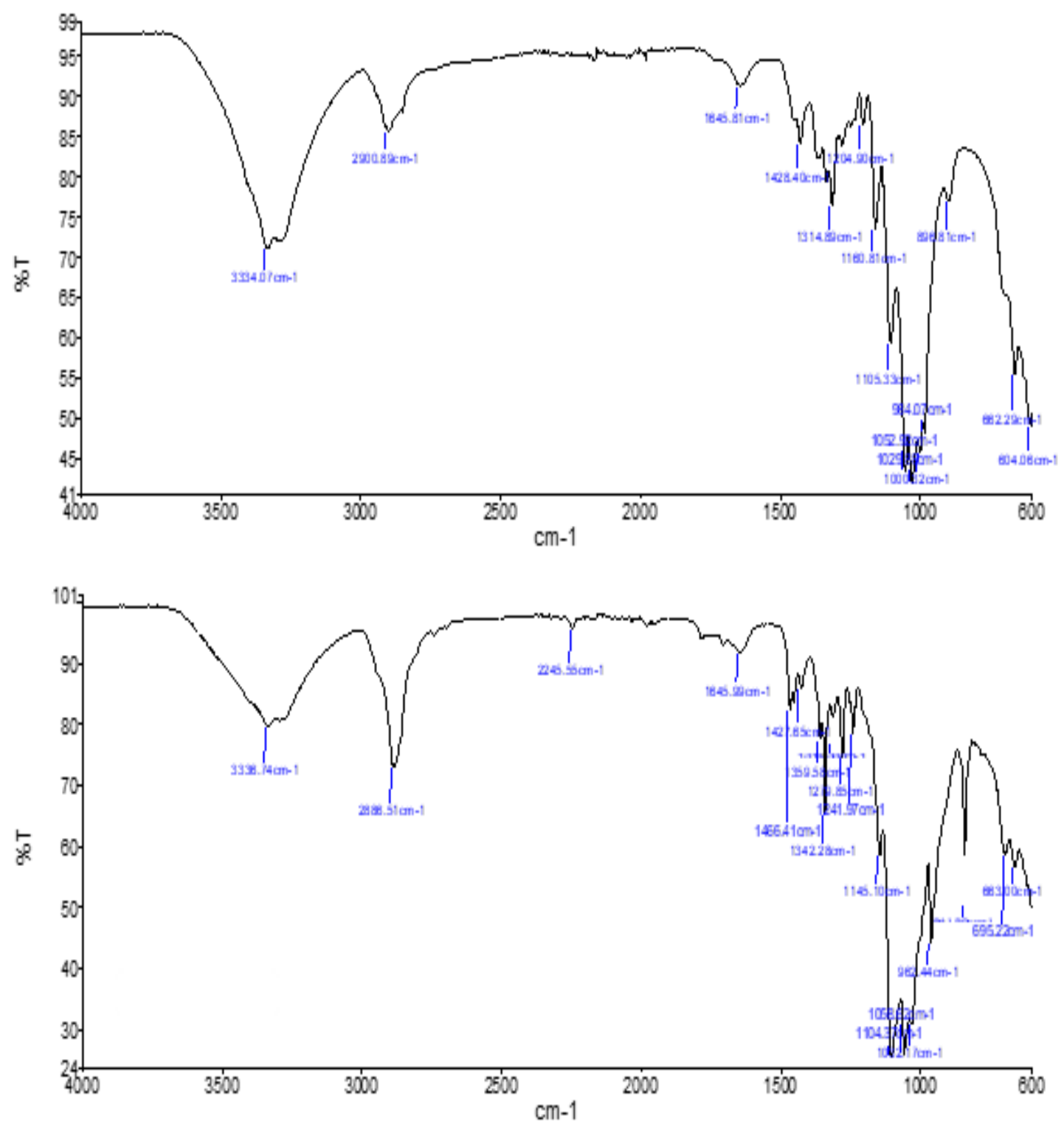

Figure 47: The top FT-IR spectrum is of uncoated cellulose fabric, the one at the bottom is of PEG FPSE media on cellulose.

In the comparison of the FT-IR spectra of uncoated cellulose and PEG FPSE media, the lack of characteristic absorption of peaks from uncoated cellulose in the spectrum for the FPSE media is once again evidence of successful bonding of the sol-gel sorbent on the surface of the substrate. Furthermore, the peaks at $2866 \mathrm{~cm}^{-1}$ and $1349 \mathrm{~cm}^{-1}$ are characteristic $\mathrm{C}-\mathrm{H}$ vibrations of PEG [10]. The 
same is true of the peaks at $1294 \mathrm{~cm}^{-1}$ and $1248 \mathrm{~cm}^{-1}$, which correspond to C-C double bonds $[10,136]$.
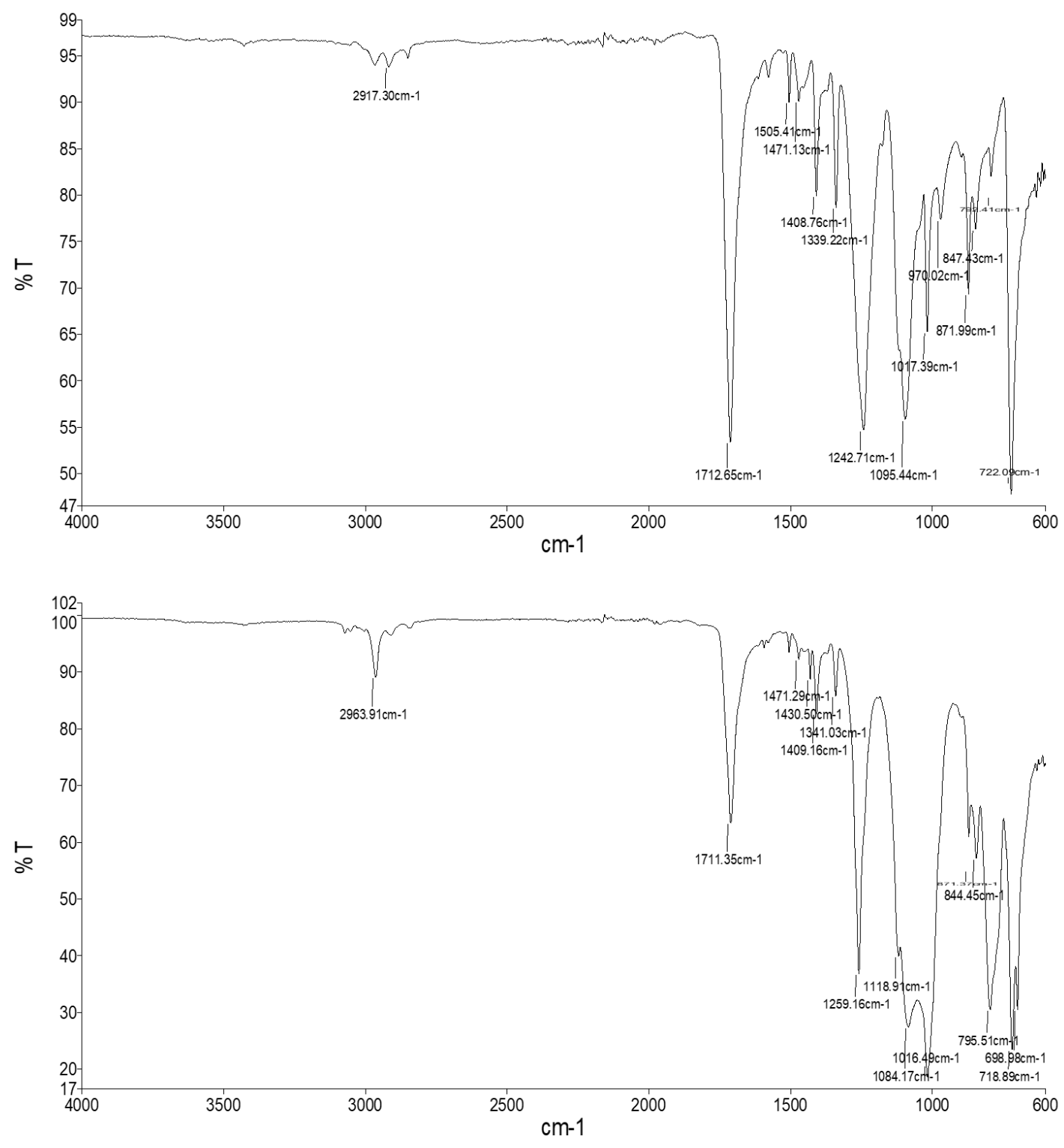

Figure 48: The top FT-IR spectrum is of uncoated polyester fabric, the one at the bottom is of PDMDPS FPSE on polyester.

Figure 48 also compares an uncoated polyester fabric to an FPSE media with that fabric as the substrate, but this time the fabric is polyester and the organic 
polymer of the sol-gel sorbent is PDMDPS. The spectrum for the uncoated polyester (top) includes a very significant absorption at $1712 \mathrm{~cm}^{-1}$, which is greatly reduced in the spectrum for the FPSE media (bottom). This is evidence that the sorbent is on the substrate. Additionally, the absorption peak at $2964 \mathrm{~cm}^{-}$ ${ }^{1}$ is also seen in the FT-IR spectrum for pure PDMDPS (Fig. 49).

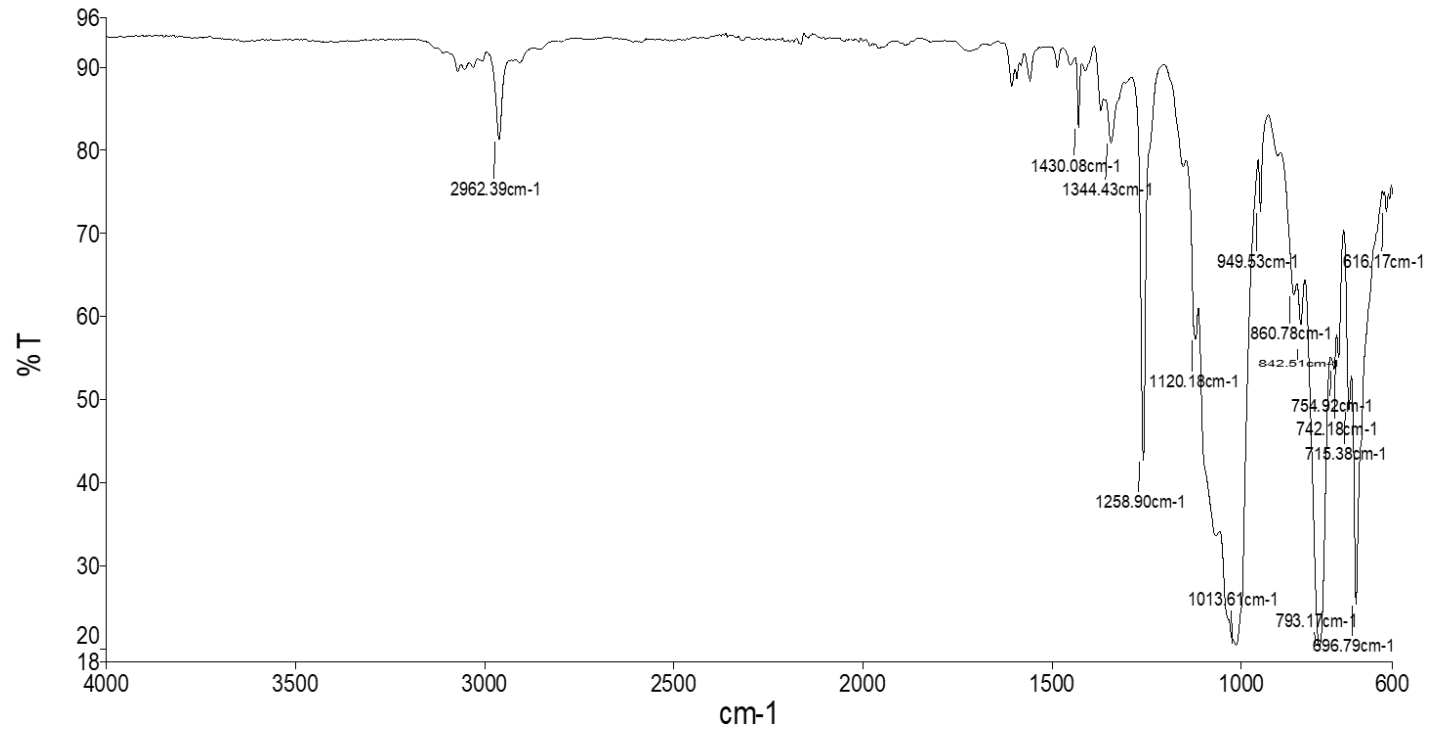

Figure 49: FT-IR spectrum of pure PDMDPS polymer.

\subsection{Nitroaromatic Explosives}

Nitroaromatic explosives are very common, which means that their production is voluminous and it is important that they are discarded properly. Safe disposal of such chemicals is not always practiced, an unfortunate reality that has caused many bodies of water close to sites of former ammunition plants to have explosive compounds in them [1]. Trace levels of these contaminants can also result indirectly from military exercises and lead to unexploded ordinances being released into oceans $[2,85,86]$. This is a problem that needs to be monitored 
because nitroaromatic explosives in environmental waters can be hazardous to wildlife in these areas, and ultimately to humans [87]. Even at trace concentrations these contaminants are potentially dangerous [5]. An FPSE method for analysis of 10 of these compounds (listed in Table 1) from environmental waters, was developed and validated.

\subsubsection{Preparation of Standard Solutions}

Six of the nitroaromatic explosive compounds: NB, 2,6-DNT, 4-NT, 3-NT, and 2NT, and 1,3-DNB were bought in dividually in bulk as solids. The other four: TNT, tetryl, TNB, 4-amino-2,6-DNT were purchased as individual 1,000 ppm standards. The solids were dissolved in methanol, to a concentration of 10,000 ppm. All the compounds were then mixed in an intermediate solution of $50 \mathrm{ppm}$ of each. This intermediate solution was used to make each standard solution for instrumental method development and subsequently for preparation of aqueous solutions to for development, validation, and application of the FPSE-HPLC-UV method.

\subsubsection{Instrumental Method Development}

The first step in the method development of the analytical procedure was to develop the instrumental method. The instrument used is an HPLC-UV. Based on published methods in the literature for similar groups of compounds, it was determined that the best solvent combination is water and acetonitrile. A method was first developed with a C-18 packed column, and while the performance was technically sufficient (resolution values of 1.5 or higher for each peak), a second 
method was developed with an Ascentis RP Amide column. The performance of this column for nitroaromatic explosives is truly outstanding, due in part to its inherent feature of forming hydrogen bonds with hydrophobic compounds such as nitroaromatic explosives. The structure of this stationary phase is show in Fig. 50:

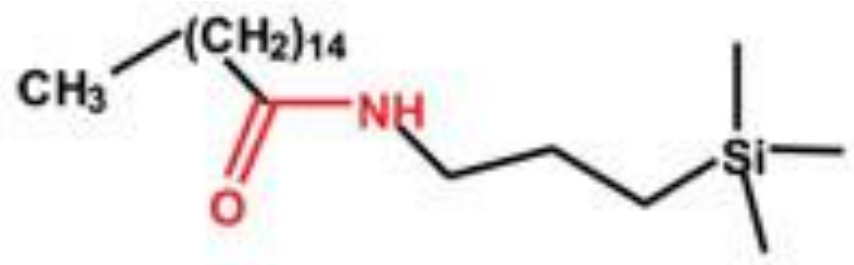

Figure 50: Structure of stationary phase of Ascentis RP Amide HPLC column. A representative chromatogram obtained from analysis of a $50 \mathrm{ppb}$ solution of the 10 pertinent nitroaromatic explosives with the developed FPSE-HPLC-UV method is show in Fig. 51. The method is isocratic, consisting of a $1.5 \mathrm{~mL} / \mathrm{min}$ flow rate, mobile phase composition of $50: 50$ water/ACN, $30^{\circ} \mathrm{C}$ column compartment temperature, $254 \mathrm{~nm}$ detection wavelength. 


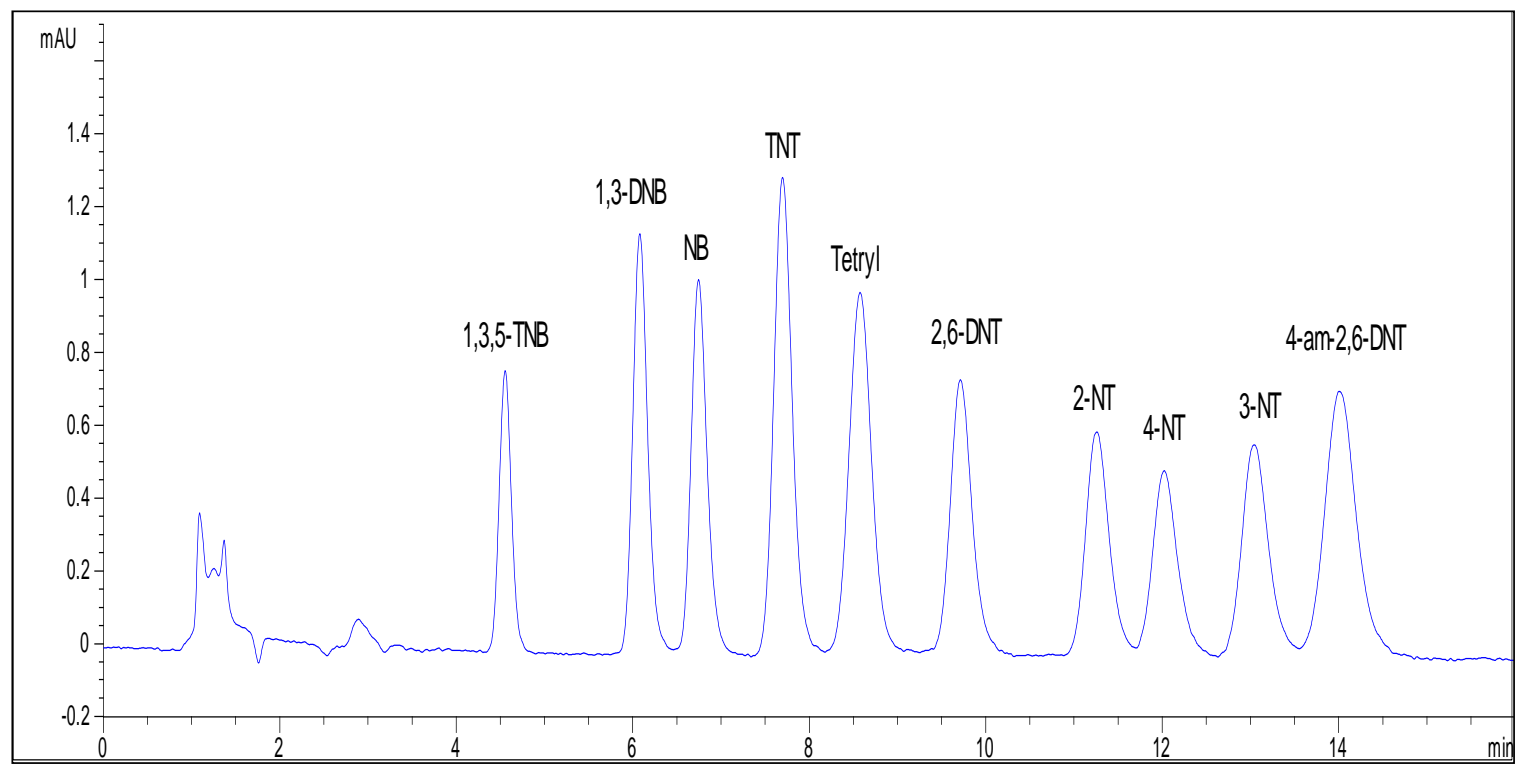

Figure 51: Representative chromatogram of developed method for 10 nitroaromatic explosives.

\subsubsection{Optimum FPSE Media Sorbent}

Once the instrumental method was ready, the next step was to determine the best sol-gel sorbent available for these compounds. The two functionalities that were compared were PTHF and PEG, each with cellulose as the sorbent. This decision was made because due to the high polarity and hydrophilicity of the nitroaromatic explosives. Sorbents with similar polarity are needed. Extractions with each were done simultaneously in triplicate from a solution in deionized water made to have a concentration of $500 \mathrm{ppb}$ of each compound. The PEG FPSE media were found to be more sensitive. This is the case because PEG is more polar than PTHF, so the partitioning of polar, hydrophilic analytes to PEG based sorbents is favored over the partitioning to PTHF sorbents. 


\subsubsection{Extraction Time Optimization}

Fabric Phase Sorptive Extraction media are used by insertion of the device in the sample, these are not devices that the sample matrix is pushed through, and as such the extractions are equilibrium based and the extraction time needs to be optimized. As stated previously, the extraction principle of FPSE is partitioning, which takes a certain amount of time that depends on a variety of factors, mainly the partition coefficient. The optimum extraction time is, in terms of overall efficiency with the priority being sensitivity, the shortest amount of time after which there is no significant increase in the amount of analyte extracted. This was tested by performing triplicate extractions from $10 \mathrm{~mL}$ aliquots of the same aqueous solution of the 10 nitroaromatic compounds of interest. The only parameter changed among the triplicate sets of extractions was the extraction time: 20, 30, 40, and 50 minutes. All other analysis parameters were unchanged throughout the study. The resulting data, shown in Fig. 52, led to the conclusion that 40 minutes is the optimum extraction time. 


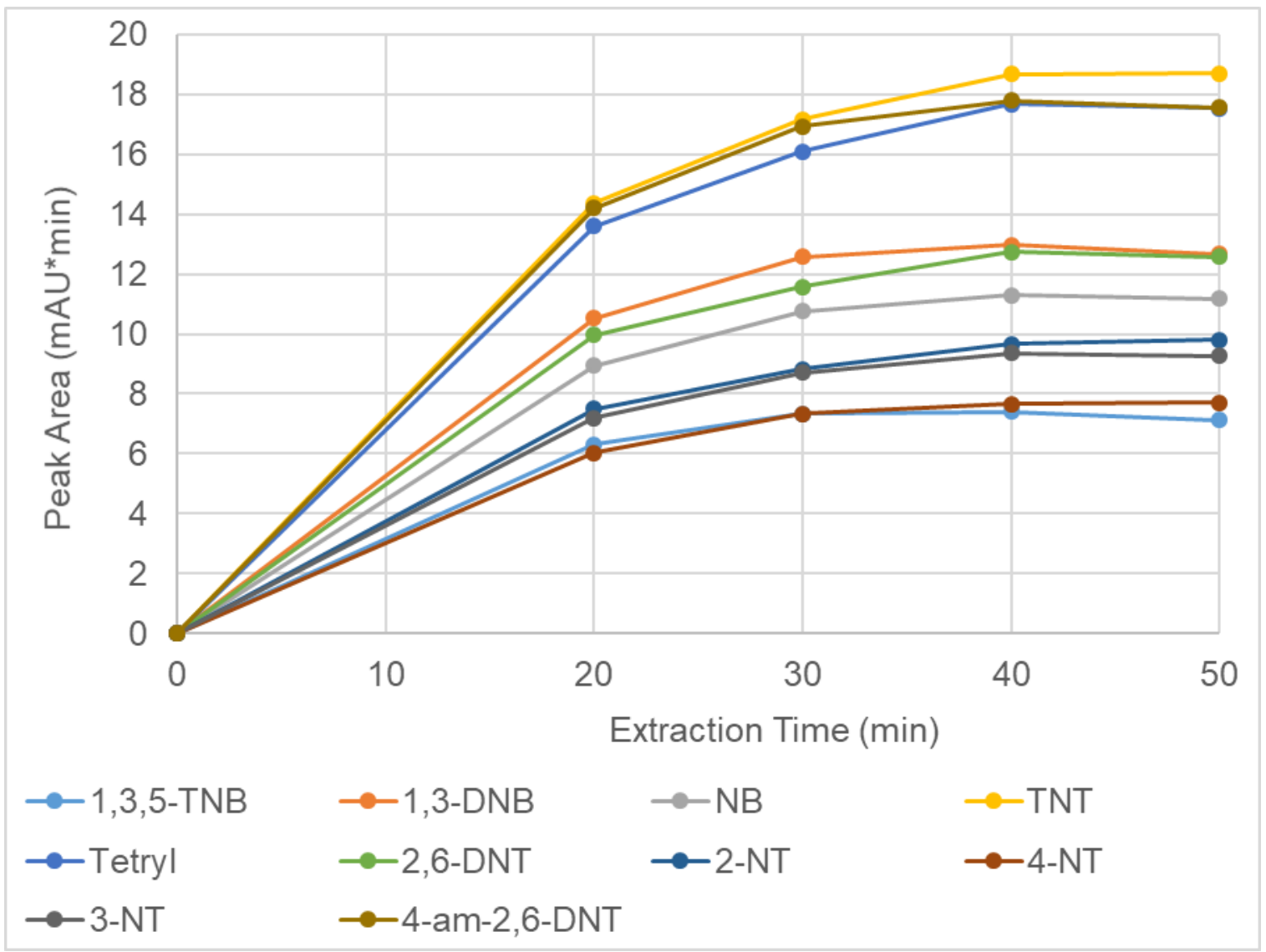

Figure 52: Experimental data of extraction time optimization for FPSE(PEG)HPLC-UV analysis of nitroaromatic explosives in water.

While the conclusion of this extraction time study was that the optimum time is 40 minutes, this may not be the case for every situation. If someone were to have a different perspective and, for example, and wants to maximize throughput instead while sacrificing some sensitivity, that person may choose 20 minutes as the optimum extraction time. It is also conceivable that someone may choose 30 minutes as the best possible extraction time if that is the shortest time that provides satisfactory sensitivity for that particular situation, saving 10 minutes in overall analysis time. 


\subsubsection{Back Extraction Time Optimization}

The back extraction is done by submerging that FPSE media, after extraction, into $500 \mu \mathrm{L}$ of organic solvent; this was not optimized because it is the lowest amount that covers most of the FPSE media when laid flat on the surface of a 10 $\mathrm{mL}$ glass vial. The time that it takes to desorb the 10 nitroaromatic explosive compounds from the FPSE media sorbent after extraction, however, was optimized in the same manner as the extraction time. Extractions of all 10 analytes were performed in triplicate, each set differing only in the time allowed for back extraction. This process happens much faster than extraction, as seen in Fig. 53:

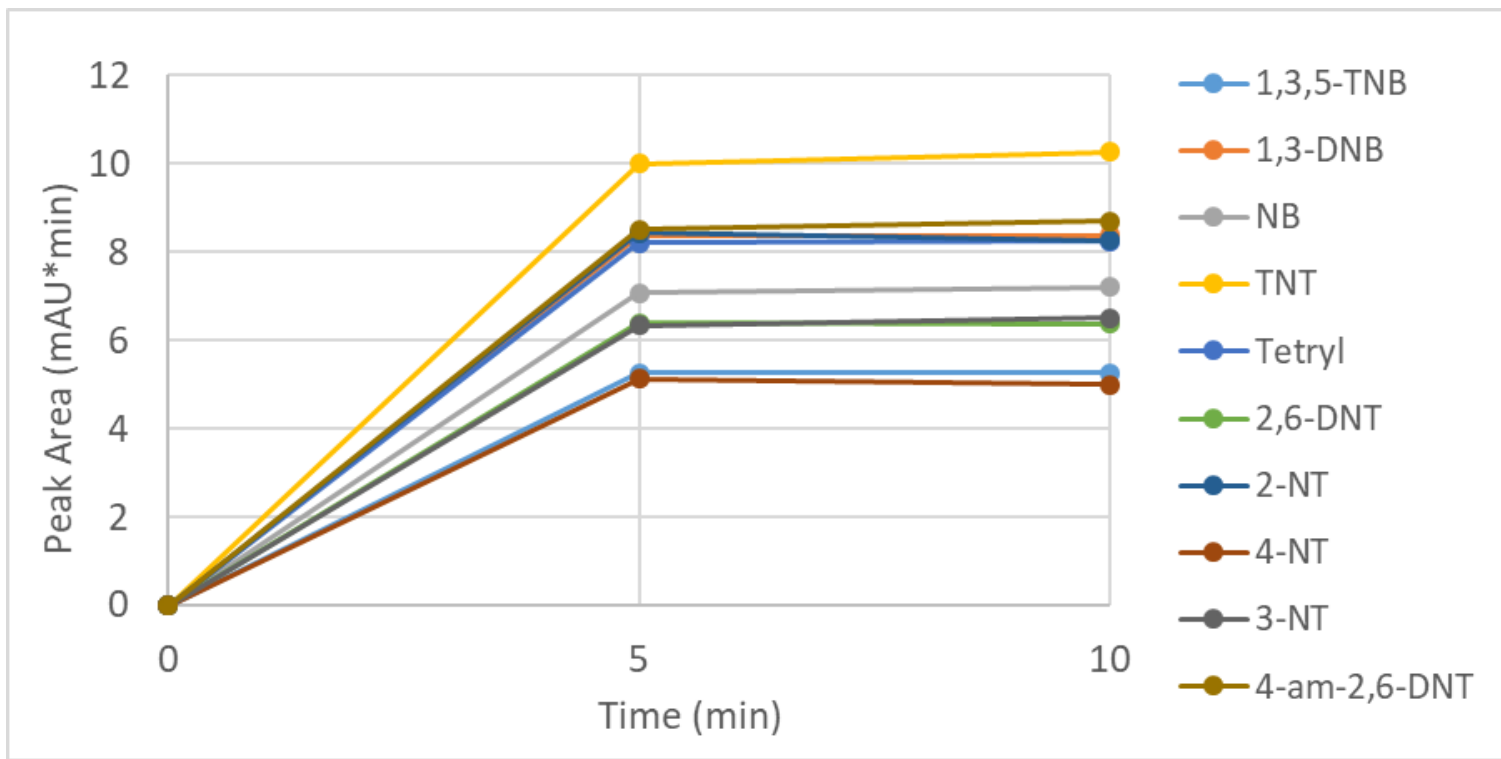

Figure 53: Experimental data of back extraction time optimization for FPSE(PEG)-HPLC-UV analysis of nitroaromatic explosives in water.

In addition to optimizing the back extraction time, the effectiveness of three solvents was investigated: methanol, acetonitrile and a 1:1 MeOH:ACN mixture. The experimental data in Fig. 54 suggests that $A C N$ and the $1: 1 \mathrm{MeOH}: \mathrm{ACN}$ 
mixture may be a better option than $\mathrm{MeOH}$, but there was no carryover detected with any of these solvent systems.

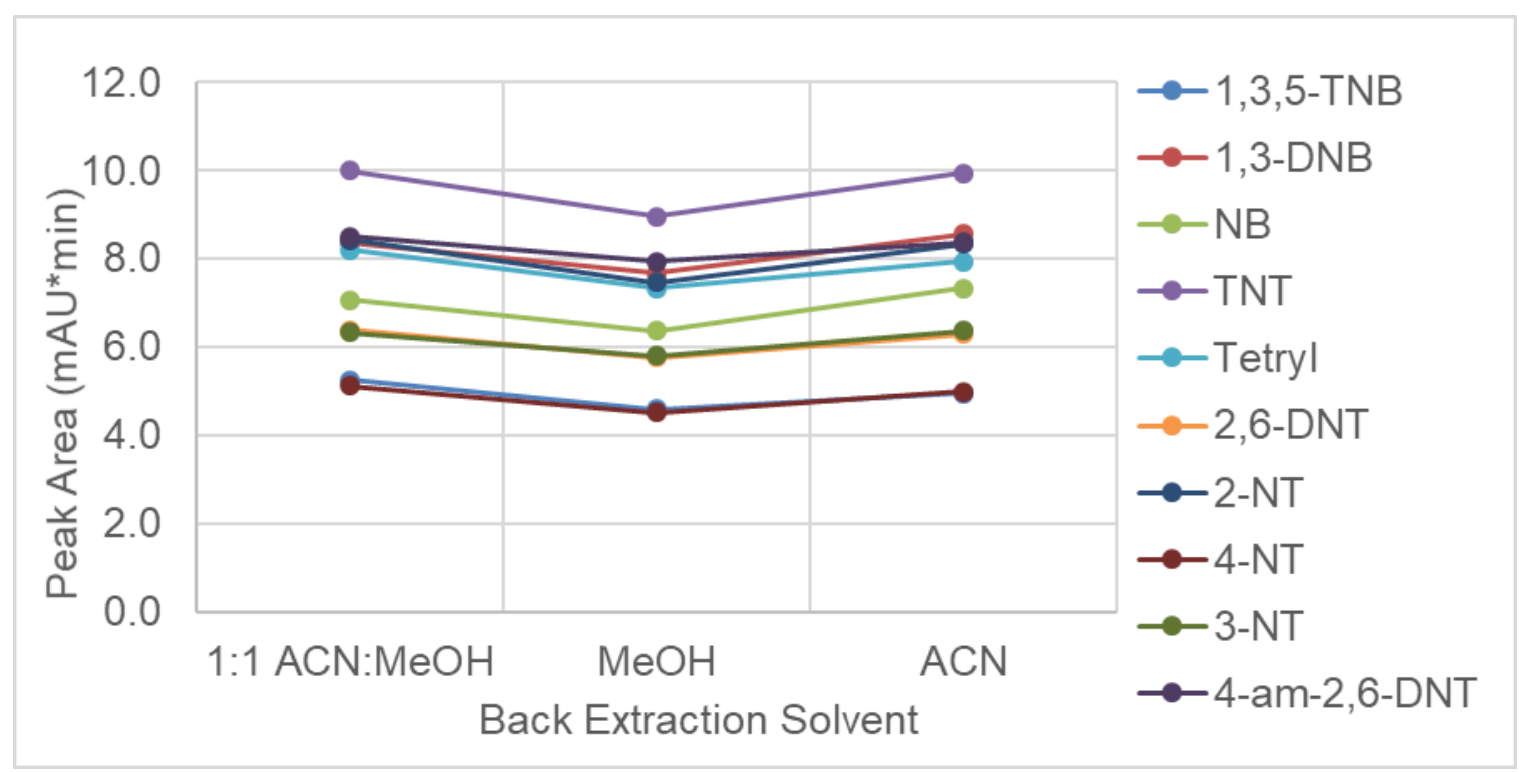

Figure 54: Experimental data of back extraction solvent optimization for FPSE(PEG)-HPLC-UV analysis of nitroaromatic explosives in water.

\subsubsection{Extraction Volume Optimization}

After determining the optimum extraction time, back extraction time and back extraction solvent, the extraction volume was optimized. This is the determination of the amount of sample after which increasing said volume does not significantly increase the amount of analyte extracted. The experimental data is shown in Fig. 55: 


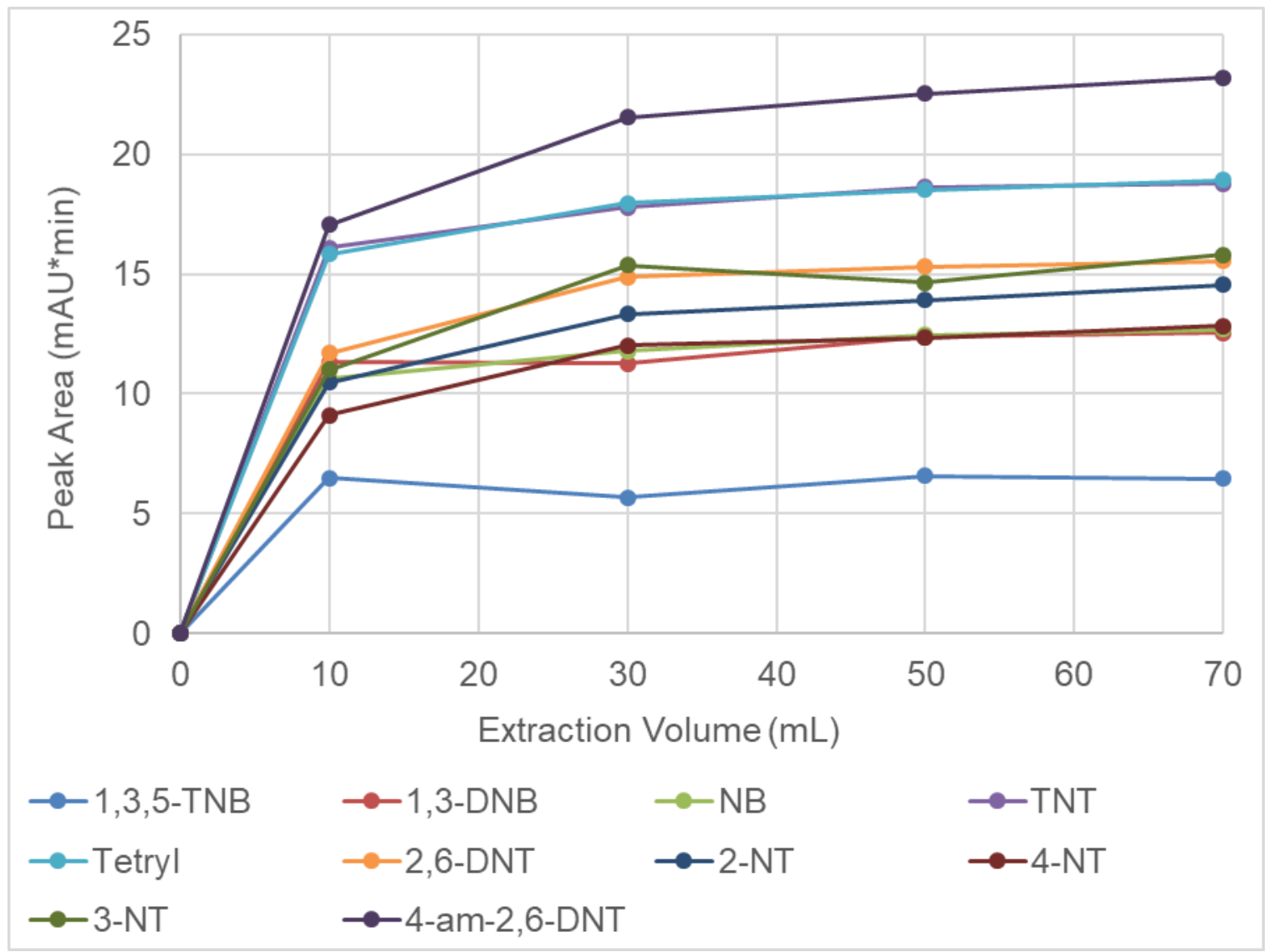

Figure 55: Experimental data of back extraction volume optimization for FPSE(PEG)-HPLC-UV analysis of nitroaromatic explosives in water.

This is a very interesting phenomenon. The optimum extraction volume was chosen to be $30 \mathrm{~mL}$, but this is arbitrary and seemingly compound specific. TNB and 1,3-DNB do not show any increase from $10 \mathrm{~mL}$ to $30 \mathrm{~mL}$ extractions, while the rest do to varying extents. Extraction volume does not make the process any longer or more difficult, unless many extractions are being done simultaneously in which case it may require more space and stirring plates. However, if there were ever a situation where the access to sample is limited in terms of quantity, there is no question that $10 \mathrm{~mL}$ is enough to obtain relatively sensitive and reliable results. 
What makes this so interesting is that this experimental result is counterintuitive because these FPSE media have much more sample loading capacity than necessary to retain all the analytes in a solution of the 10 nitroaromatic explosives at $50 \mathrm{ppb}$, and even at $70 \mathrm{~mL}$. The mass of each of the compounds in solution is $3.5 \mu \mathrm{g}\left(70 \mathrm{ng} / \mathrm{mL}^{*} 50 \mathrm{~mL}\right)$, which amounts to a total mass of $35 \mu \mathrm{g}$ total (3.5 $\mu \mathrm{g} /$ analyte * 10 analytes). The sample loading capacity of PEG FPSE media is considerably greater: $8.63 \mathrm{mg} / \mathrm{cm}^{2}$, or $43.2 \mathrm{mg} / \mathrm{unit}\left(5 \mathrm{~cm}^{2}\right)$. This is a breakthrough amount compared to the alternatives, made possible by the inherent porosity of sol-gel materials, the nature of the design with the sorbent being spread out over a planar surface, and having sorbent being coated on both sides.

From the point of view of the extraction mechanism there is no further explanation of this phenomenon. According to Equation 3, on Pg. 27, the partitioning coefficient is given by the ratio of the compound in the sorbent over the sample. As the volume of the sample is increased, the amount of analytes is increased proportionally, because it is simply a larger amount of the same solution. The volume of sorbent is not increased, because the FPSE media are kept at $5 \mathrm{~cm}^{2}$. Let's take a closer look at the extractions of $10 \mathrm{~mL}$ of solution vs. 30. If the partitioning coefficient were to remain constant, the response would be linear: the concentration in the sorbent would be 3 times the concentration in the sorbent for the $10 \mathrm{~mL}$ extraction and the concentration in the sample would be the same. However, this is not what actually happens, which serves as a reminder that experiments need to be carried out to prove hypotheses. The only 
reasonable conclusion, based on the experimental data, is that the volume is a determining factor in the partition coefficient. This is a very important determination because it means that lower volumes are more suitable for quantitation. Therefore, the optimum extraction volume for circumstances when quantitation is the priority is $10 \mathrm{~mL}$ of sample, not 30 .

\subsubsection{Linearity, Inter and Intraday Repeatability}

The developed FPSE(PEG)-HPLC-UV method is intended for quantitation in addition to qualitative analysis. Whenever a method is to be used for quantitation, it is essential that the dynamic range be determined. This is the range of concentrations that produce proportional responses upon analysis by the method, forming a linear relationship. The data presented in Fig. 56 shows the results of the linearity study. Sets of triplicate extractions were done, the only difference among the sets being the concentration of solution: $10,25,200,500$, and $1000 \mathrm{ppb}$. The solutions were prepared by serial dilution to minimize error. The high end of the dynamic range was not determined beyond $1 \mathrm{ppm}$ because it is not realistic to think that such concentrations would be encountered in real environmental samples. 


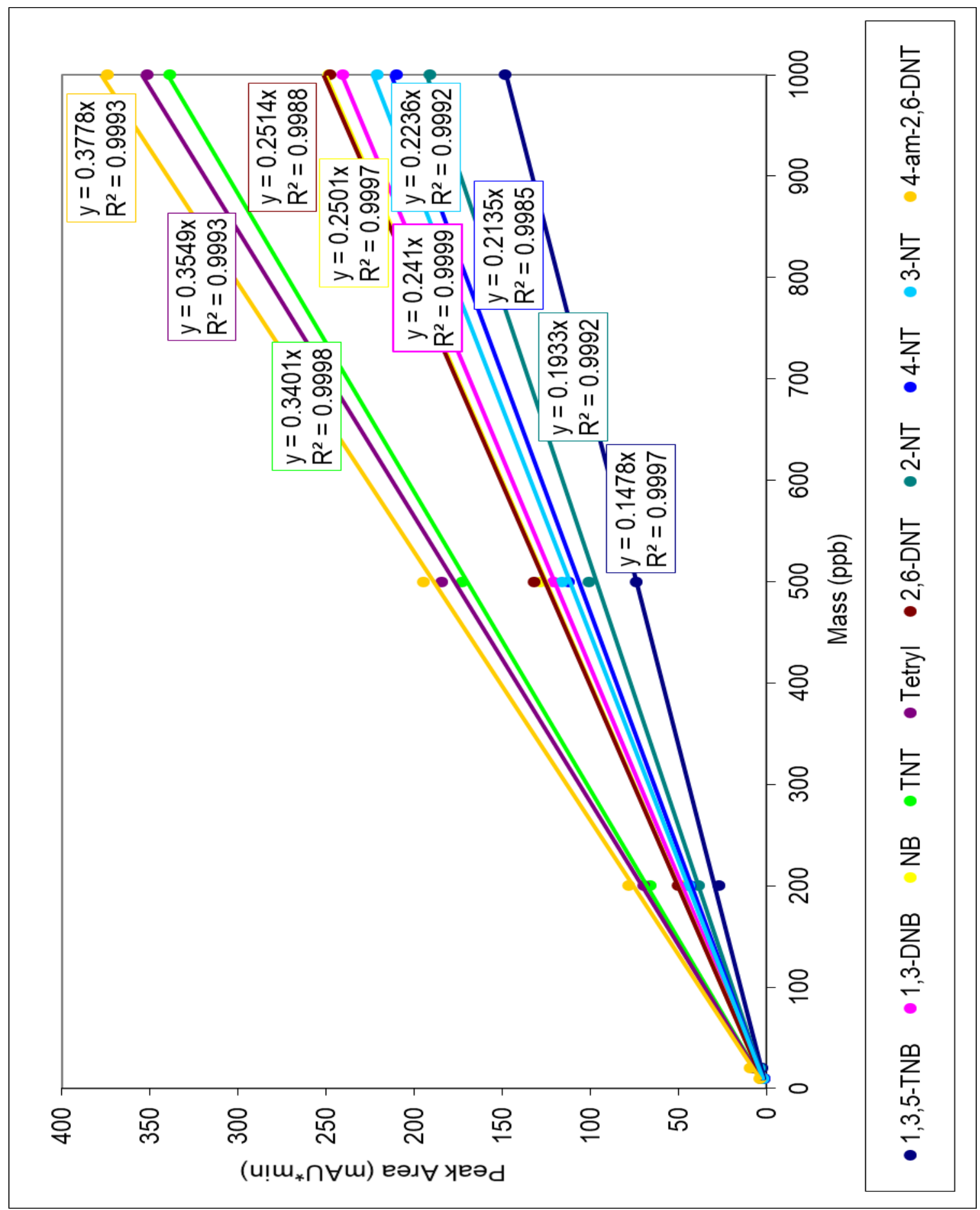

Figure 56: Experimental data for determination of compound specific linear range for the developed FPSE(PEG)-HPLC-UV method for analysis of nitroaromatic explosives in water. 
Repeatability is important for any method in analytical chemistry, especially when quantitation is a priority. The repeatability of the developed FPSE(PEG)-HPLCUV method was measured both intra and inter-day. The intraday repeatability measures whether the method can be expected to deliver consistent performance over the span of a workday. This was measured for each analyte by performing 5 separate analysis of $10 \mathrm{~mL}$ aliquots of the same $50 \mathrm{ppb}$ solution over the course of one day.

Inter-day repeatability is also a measure of whether the method can be expected to deliver consistent performance, but this time it is tested over three days. Triplicate extractions of the same solution that was used for intraday repeatability were done, one set per day over three days. For both kinds of repeatability studies the unit of measure was Percent Relative Standard Deviation (\% RSD), which is commonly used for this purpose. Table 6 presents a comprehensive summary of the results of the linearity study as well as both repeatability studies. The data is given for each nitroaromatic explosive compound. 
Table 6: Summary of the compound specific results for intra and inter-day repeatability as well as dynamic range and the corresponding correlation coefficient for the developed FPSE(PEG)-HPLC-UV method for the analysis of nitroaromatic explosives in water.

\begin{tabular}{|c|c|c|c|c|}
\multirow{2}{*}{ Compound } & \multicolumn{2}{|c}{ Repeatability (\% RSD) } & \multicolumn{2}{c|}{ Linearity } \\
\cline { 2 - 5 } & Intraday & Inter-day & Range $(\mathrm{ppb})$ & $\begin{array}{c}\text { Correlation } \\
\left(\mathrm{R}^{2}\right)\end{array}$ \\
\hline $\mathbf{1 , 3 , 5 - T N B}$ & 7.0 & 2.7 & $10-1000$ & 0.9997 \\
\hline $\mathbf{1 , 3 - D N B}$ & 4.8 & 3.3 & $10-1000$ & 0.9999 \\
\hline NB & 3.1 & 2.9 & $10-1000$ & 0.9997 \\
\hline TNT & 3.5 & 2.5 & $10-1000$ & 0.9998 \\
\hline Tetryl & 4.1 & 3.5 & $10-1000$ & 0.9993 \\
\hline 2,6-DNT & 2.8 & 2.6 & $10-1000$ & 0.9988 \\
\hline 2-NT & 2.3 & 1.5 & $10-1000$ & 0.9992 \\
\hline 4-NT & 3.1 & 1.4 & $10-1000$ & 0.9985 \\
\hline 3-NT & 3.9 & 1.5 & $10-1000$ & 0.9992 \\
\hline 4-am-2,6-DNT & 3.0 & 1.8 & $10-1000$ & 0.9993 \\
\hline
\end{tabular}

Figure 56 and Table 6 both show outstanding experimental linearity results for each compound. The dynamic range covers three orders of magnitude, starting at a very low concentration considering that the analytes are highly polar and hydrophilic and the sample matrix is water. The repeatability studies also resulted in consistently satisfactory values that are well within accepted margins of error. 


\subsubsection{Limits of Detection and Quantitation}

The sensitivity of analytical methods is measured according to the Limits of Detection and Quantitation (LOD and LOQ, respectively). This is a special figure of merit when evaluating a method because it influences both qualitative and quantitative analysis. The LOD refers to the lowest concentration that can be detected with certainty; LOQ is the lowest concentration that produces a large enough response that it can be quantified accurately. While there are various formulations for calculation of these quantities and disagreement as to the legitimacy, accuracy and practical implications of each, there are two that are widely accepted. In this case, the LOD of the developed FPSE(PEG)-HPLC-UV method for analysis of nitroaromatic explosives from aqueous samples, was calculated by dividing the slope of the corresponding line in the dynamic range curve by the noise and multiplying it by 3 . The LOQ was calculated dividing the slope of the corresponding line in the dynamic range curve by the deviation in the peak-to-peak noise from method blanks and multiplying it by 10 .

The other widely accepted methodology for calculating LOD and LOQ concerns the signal-to-noise ratio of each compound in the corresponding method and the concentration. Peak-to-peak is one of two accepted methods for noise determination in chromatography. As shown in Fig. 57 [137], this measures the distance between low and high peaks within the noise in a given segment of the chromatogram, then takes the average of all these differences. 


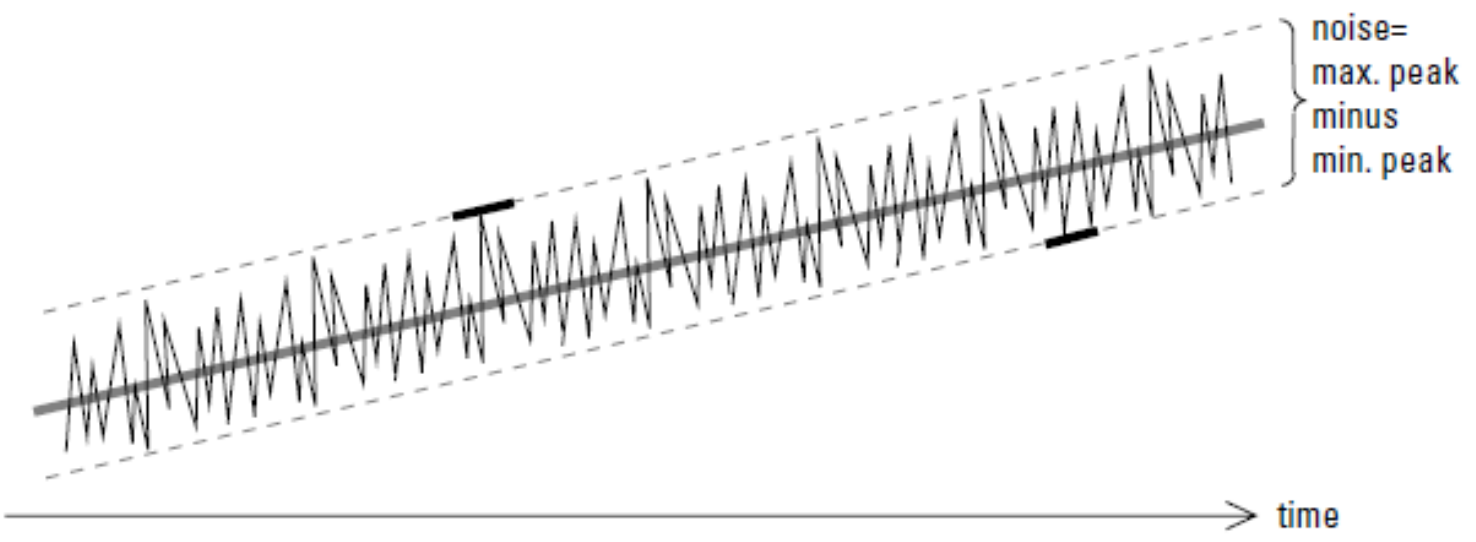

Figure 57: Schematic demonstrating how peak-to-peak noise distances are measured.

The LOD values were compared with those published in two separate peerreviewed articles from different authors. The method published by Malik et al. [138] is a direct immersion SPME-HPLC-UV methods for analysis of nitroaromatic explosives from aqueous samples and did not achieve the levels of sensitivity procured by the developed method presented here.

The one published by Valcárcel et al. is a D- $\mu S P E-U P L C-D A D$ method [139]. D$\mu$ SPE stands for Dispersive Micro Solid Phase Extraction, which is a derivative of traditional SPE that uses nanoparticles (small clusters of atoms or molecules) to increase the PCSA of the stationary phase [139]. As discussed previously, having a relatively low PCSA is one of the deficiencies of traditional SPE. However, even with this modification, the method developed does not provide LOD values as low as those obtained with the developed FPSE based method being introduced. All results pertinent to LOD and LOQ values, including those found in the literature are shown in Table 7. 
Table 7: Compound specific results for experimental calculations of LOD and

LOQ for the developed FPSE(PEG)-HPLC-UV method and comparison to performance of methods in the literature. N/A signifies that the compound was not included in the research article.

\begin{tabular}{|c|c|c|c|c|}
\hline Compound & LOD (ppt) & LOQ (ppt) & $\begin{array}{c}\text { LOD (ppt) } \\
\text { Malik et al. }\end{array}$ & $\begin{array}{c}\text { LOD (ppt) } \\
\text { Valcárcel et } \\
\text { al. }\end{array}$ \\
\hline 1,3,5-TNB & 80 & 267 & 920 & N/A \\
\hline 1,3-DNB & 243 & 810 & 470 & N/A \\
\hline NB & 234 & 781 & 370 & 460 \\
\hline TNT & 130 & 432 & 350 & N/A \\
\hline Tetryl & 124 & 414 & 540 & N/A \\
\hline 2,6-DNT & 47 & 156 & 170 & $530^{*}$ \\
\hline 2-NT & 157 & 524 & 280 & N/A \\
\hline 4-NT & 142 & 475 & 470 & 1260 \\
\hline $\begin{array}{c}\text { 3-NT } \\
\text { 4-am-2,6- } \\
\text { DNT }\end{array}$ & 15 & 49 & 400 & 810 \\
\hline \begin{tabular}{c} 
*LOD value for 2,4-DNT, not 2,6-DNT. LOD value for 2,6-DNT with that same \\
\hline
\end{tabular}
\end{tabular}

\subsubsection{Field Application to Environmental Samples}

Upon development of the method and determination of the figures of merit, the last goal was to apply it to real samples and simultaneously test its effectiveness as a portable method. The first application was analysis of a sample of water from a pond at FIU's Modesto Maidique Campus. A sample was collected and not pretreated in any way before analysis. Another sample was collected and 
spiked to a concentration of $100 \mathrm{ppb}$ of each nitroaromatic explosive. The intermediate solution containing all 10 analytes was transported to the field in a cooler with ice. Both samples, one spiked and one pristine, were analyzed beside the pond and also at the laboratory 24 hours later. This was done to determine whether the effectiveness of the method is as good when the sample preparation portion of it is done in the field. In other words, are PEG FPSE media legitimately portable? The experimental data is shown in Fig. 58.

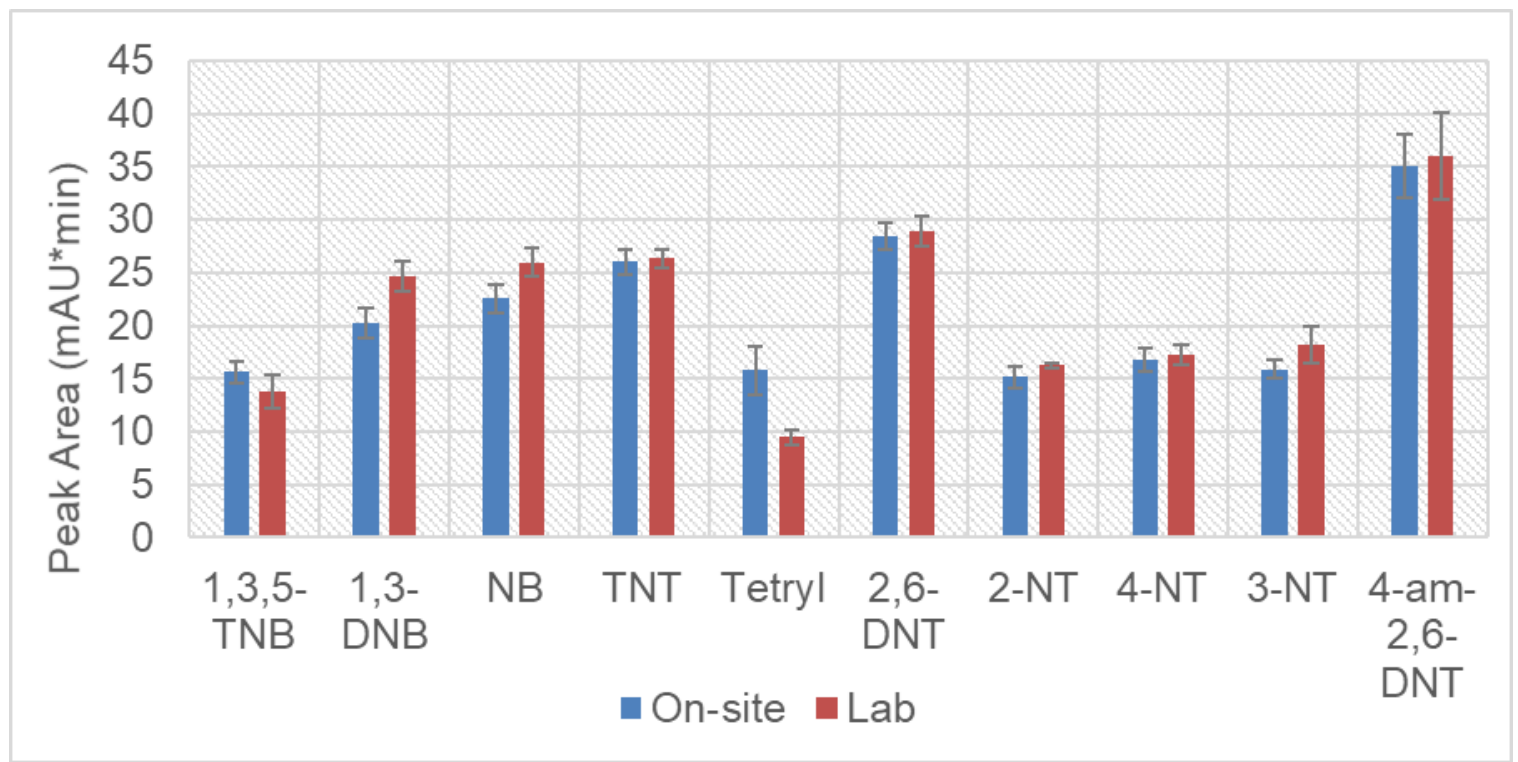

Figure 58: Experimental data pertaining to the comparison of the effectiveness of the developed FPSE(PEG)-HPLC-UV method at an FIU pond vs. analysis of the same sample transported to the laboratory.

The same concept and experimental design was used to test the method on saline water from nearby Key Biscayne Beach. This was done because seawater bodies are susceptible to contamination with this kind of explosive compounds, just like fresh water bodies like ponds and lakes. The results are presented in Fig. 59: 


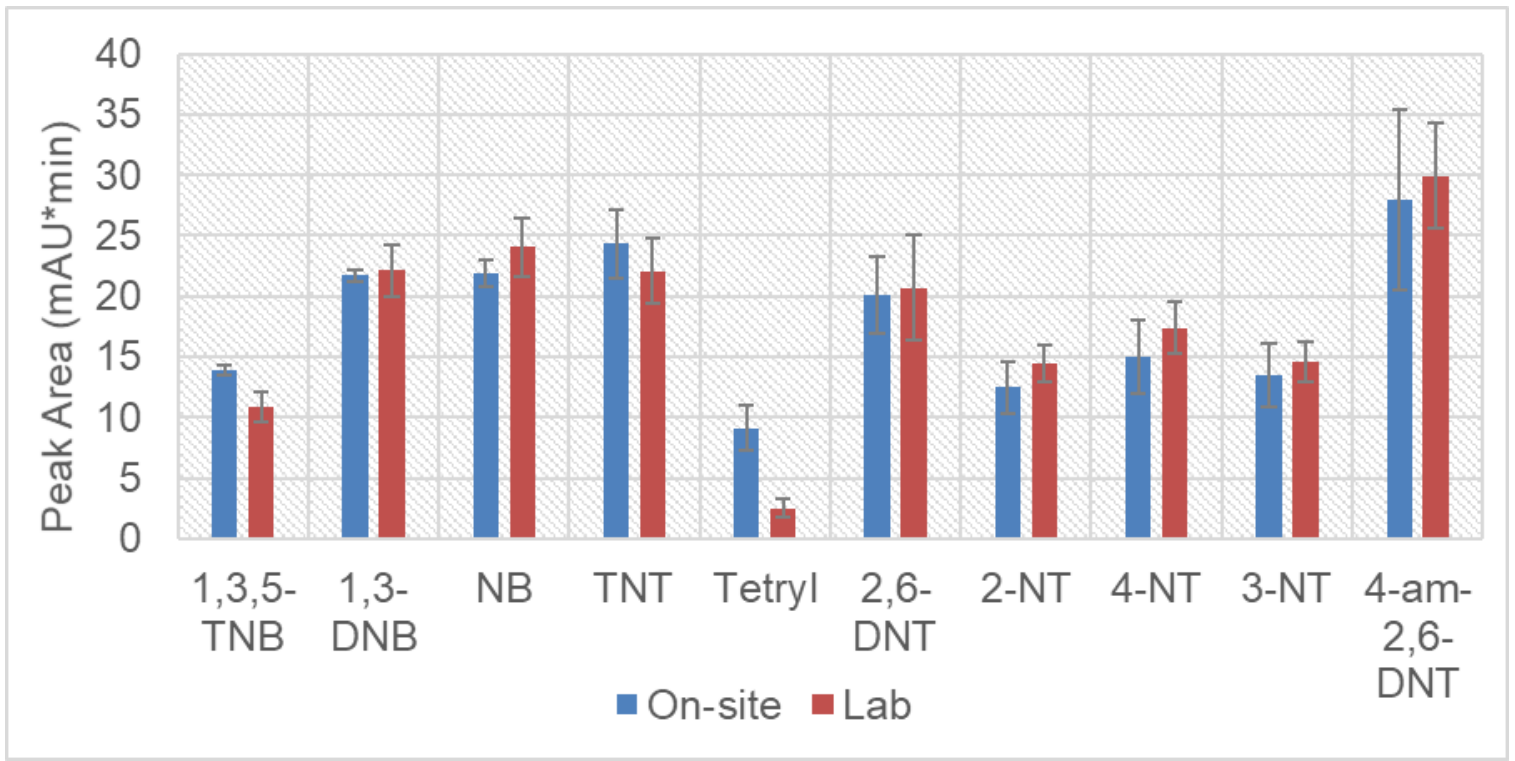

Figure 59: Experimental data pertaining to the comparison of the effectiveness of the developed FPSE(PEG)-HPLC-UV method at Key Biscayne Beach vs. analysis of the same sample transported to the laboratory.

Figs. 58 and 59 present very similar data. The effectiveness of the extractions on the field is equivalent to that of the analysis of the same solutions in the laboratory for all analytes except tetryl. For tetryl specifically the analysis is more effective in the field, perhaps due to consumption of this compound by microorganisms. These two studies clearly show that the developed FPSE(PEG)HPLC-UV method is not only convenient, rugged, green, reliable, and more sensitive than those in the literature with similar instrumentation, it is also full deployable in the field which provides a plethora of advantages. All of these are characteristics of ideal sample preparation techniques.

5.4 Benzene, Toluene, Ethylbenzene, Xylenes

All BTEX compounds are carcinogenic, so much so that the United States imposed the maximum air concentration of benzene at $1 \mu \mathrm{g} / \mathrm{m}^{3}$ [104]. Not only 
are these compounds very toxic, there is a wide variety of entry points into the environment that in modern societies are impossible to eliminate: normal working processes of power plants [6], petroleum derivatives [105], automobile exhaust engines [7], and even domestic use of heaters and gas boilers [106,107]. While this amount of common sources of BTEX compounds may feel overwhelming, it does not necessarily mean that the environment is or will be contaminated to high enough levels to be unfit for human life. It certainly makes it imperative, however, that regulatory methods be able to reliably quantify their presence in environmental water at ultra-trace levels of concentration. An FPSE based method is presented subsequently for analysis of environmental water sources for 5 BTEX compounds (listed in Table 2).

\subsubsection{Preparation of Standard Solutions}

Benzene, toluene, ethylbenzene, o- and p-xylene were bought individually in bulk liquid form. Stock solutions were made of each with acetonitrile as the solvent, the concentration being $10,000 \mathrm{ppm}$. From these very highly concentrated standard solutions a 100 ppm intermediate of all 5 analytes was made and subsequently used for instrumental method development, preparation of standard solutions in deionized water and spiking of environmental samples as needed.

\subsubsection{Instrumental Method Development}

For analysis of BTEX from aqueous matrices the instrument of choice was HPLC-UV. These compounds all have a benzene ring which exhibits UV 
absorption because of its conjugation. The HPLC column's stationary phase was

C-18. The instrumental method parameters were as follows: 50:50

water:acetonitrile mobile phase composition, $1 \mathrm{~mL} / \mathrm{min}$ flow rate, $30^{\circ} \mathrm{C}$

temperature control in column compartment, $195 \mathrm{~nm}$ detector wavelength. A

representative chromatogram of FPSE-HPLC-UV analysis of a $10 \mathrm{ppb}$ solution of the 5 BTEX compounds in deionized water is presented in Fig. 60.

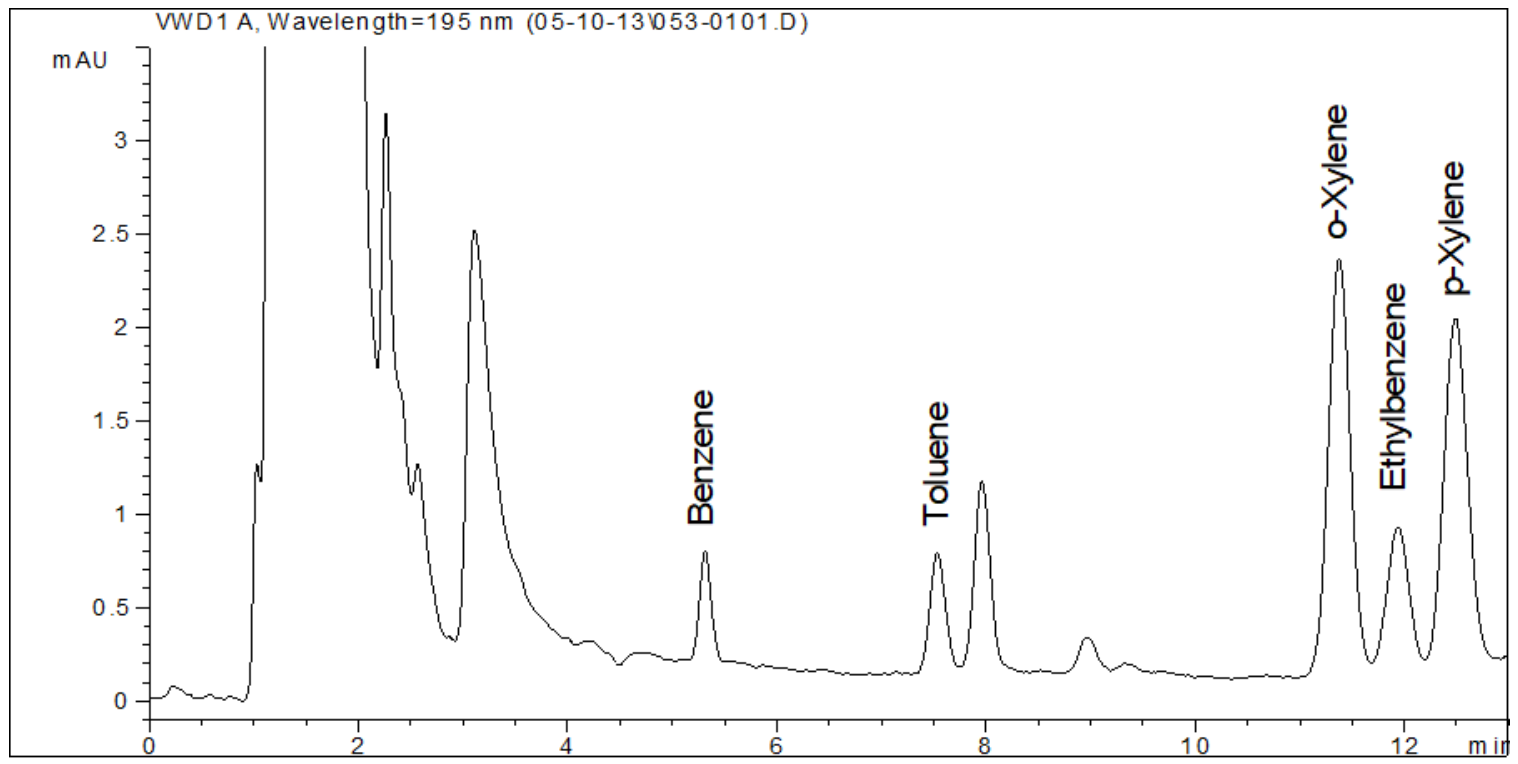

Figure 60: Representative chromatogram of analysis of a $10 \mathrm{ppb}$ solution of the 5 pertinent BTEX compounds from deionized water with the developed FPSEHPLC-UV method.

\subsubsection{Determination of Optimum FPSE Media Sorbent}

The first step in method development is always developing the instrumental method. For FPSE-based methods, the second step is always determining the optimum sorbent available. Which sorbent offers the most favorable partition coefficient? For extraction of BTEX compounds the effectiveness of two sorbents 
was compared: PTHF and PDMDPS. These two sorbent chemistries were chosen because BTEX compounds are pure hydrocarbons, which translates to them being slightly polar or nonpolar. As can be seen Table 2, the octanol-water partition coefficients for these compounds range from 2.1 - 3.3. PTHF is a slightly polar polymer, while PDMDPS has phenyl groups that may retain BTEX compounds by pi-pi interactions. The results of this comparison can be seen in Fig. 61. Based on this results the decision was made to develop the method with FPSE-PTHF media.

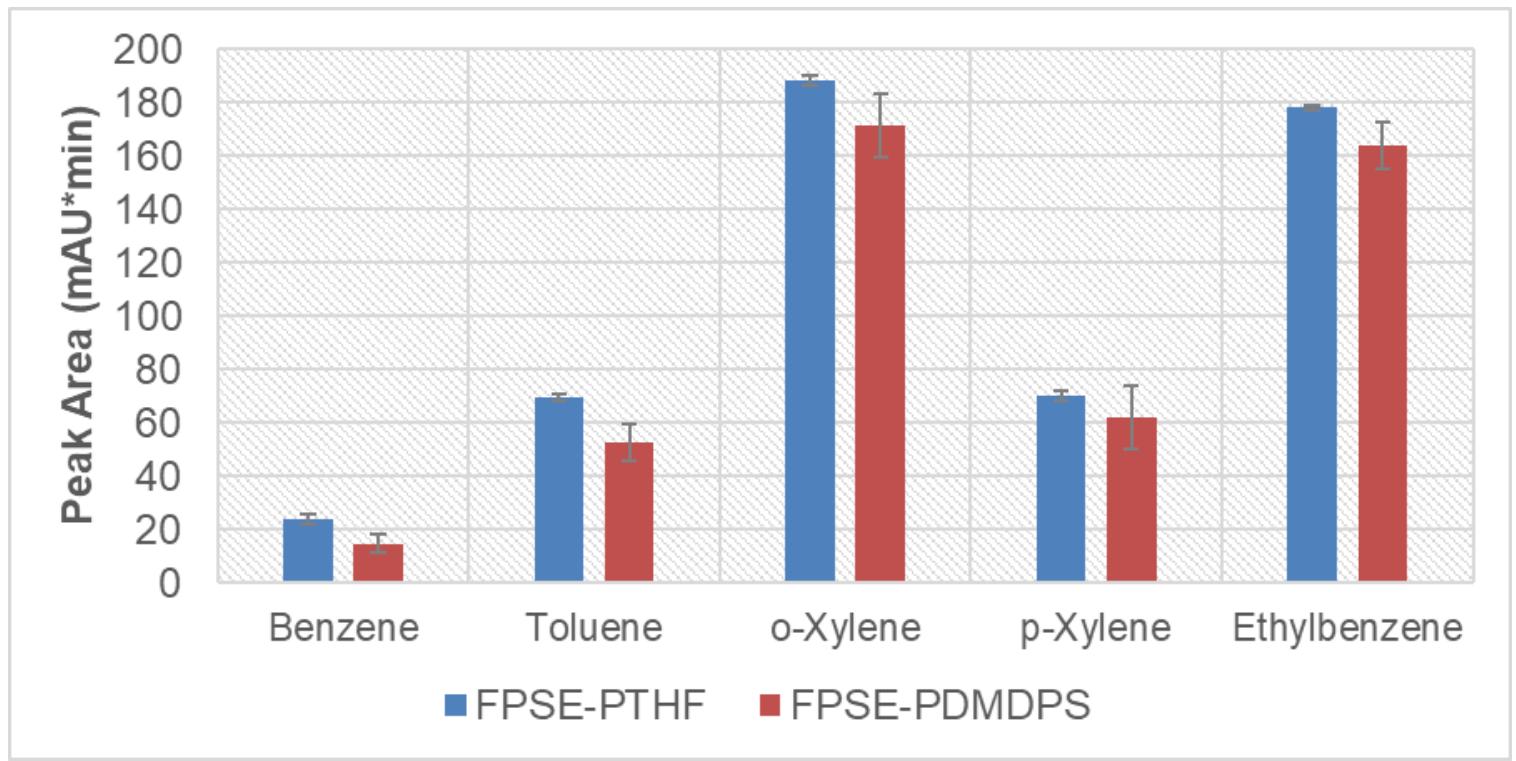

Figure 61: Experimental data of the comparison of the effectiveness of FPSEPTHF and FPSE-PDMDPS media, respectively, for extraction of BTEX compounds from aqueous matrices.

\subsubsection{Optimization of Sample Preparation Parameters}

The first sample preparation parameter that was optimized was the extraction time. The same design that was used for optimization of the extraction time for 
analysis of nitroaromatic explosives was followed again. Figure 62 shows the experimental data.

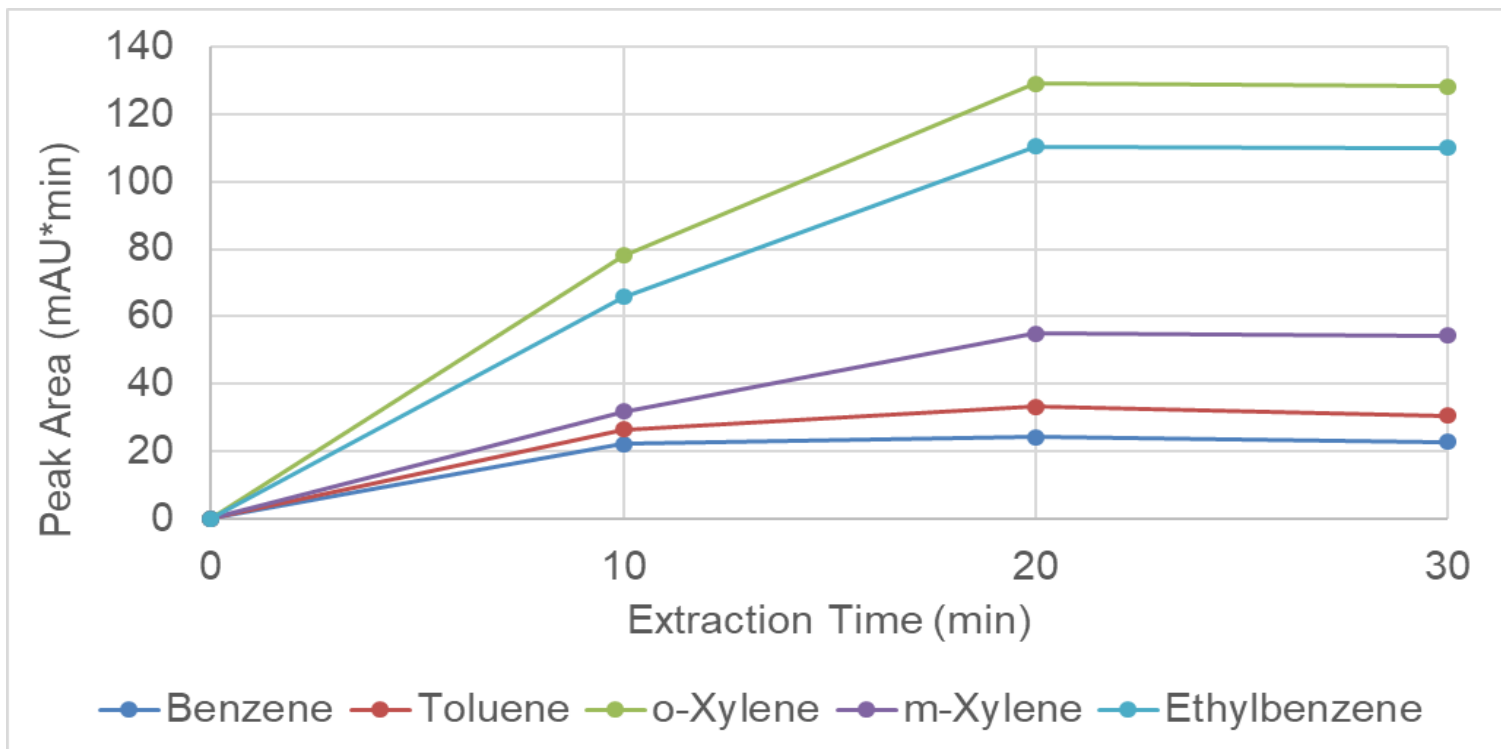

Figure 62: Experimental data of extraction time optimization of the FPSE(PTHF)HPLC-UV being developed for analysis of BTEX compounds in aqueous samples.

The optimum extraction time, based on the preceding data, was chosen to be 20 minutes, although as discussed previously, this is arguable depending on the needs and priorities of the user. This decision was made such that the sensitivity of the method is maximized.

The back extraction time and solvent were optimized after the extraction volume. This was also done with the same design used for optimization of these method parameters in the nitroaromatic explosives project. Figs. 63 and 64 present the experimental data for optimization of the back extraction time and volume. 


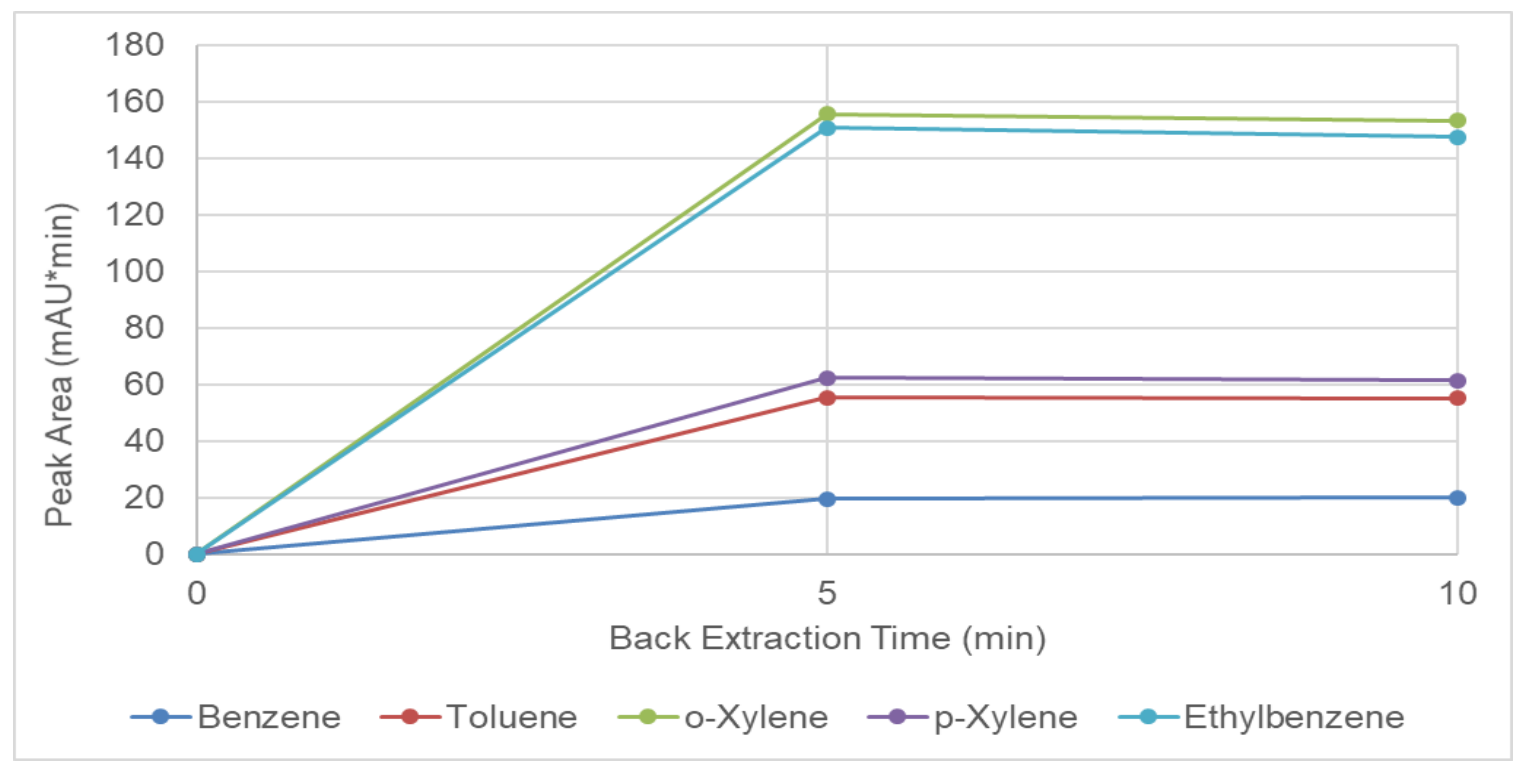

Figure 63: Experimental data of back extraction time optimization of the FPSE(PTHF)-HPLC-UV being developed for analysis of BTEX compounds in aqueous samples.

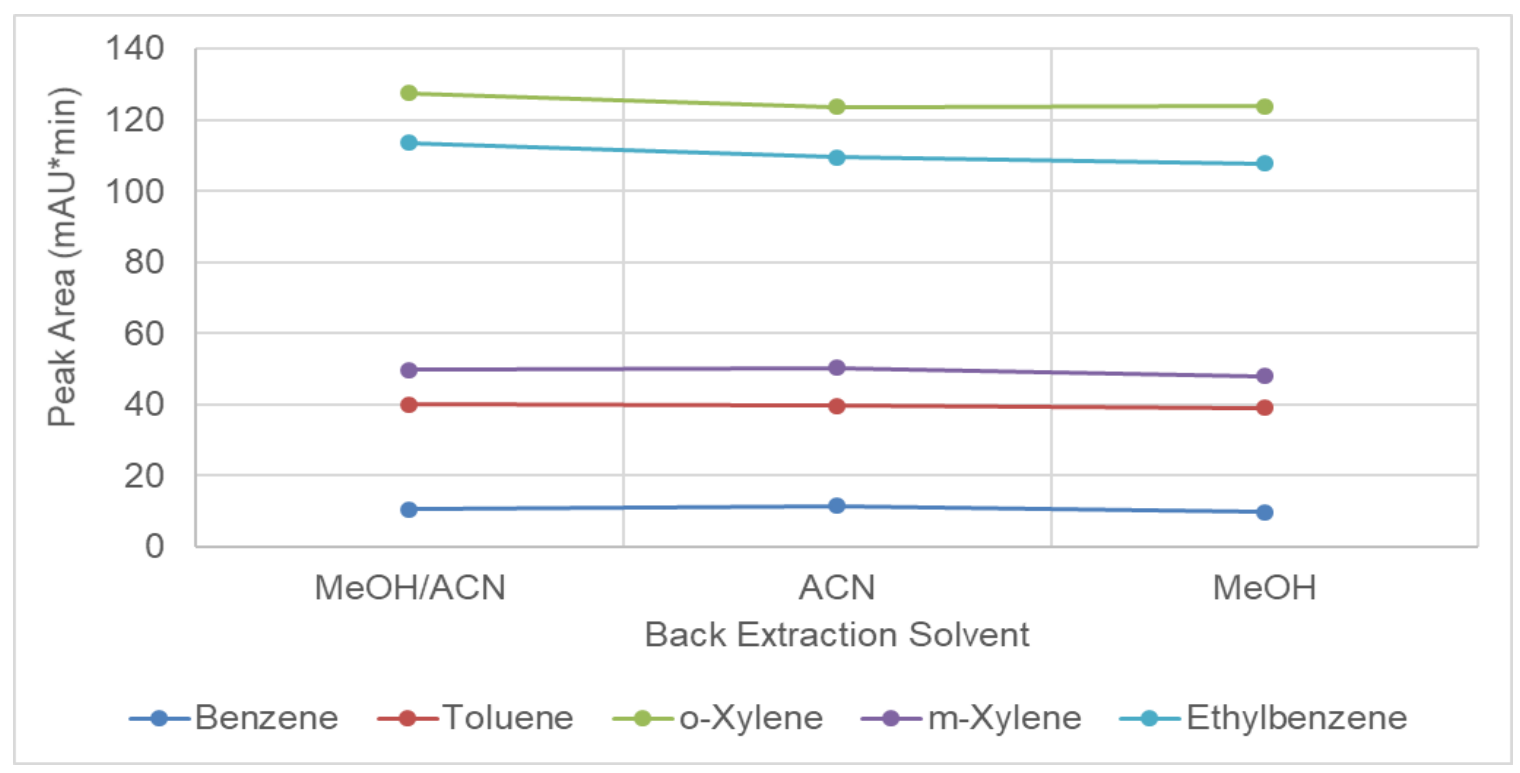

Figure 64: Experimental data of back extraction solvent optimization of the FPSE(PTHF)-HPLC-UV being developed for analysis of BTEX compounds in aqueous samples. 
The analysis of the data in both Figs. 63 and 64 is not difficult. The optimum back extraction time is 5 minutes; the three extraction solvents investigated, methanol, acetonitrile and an equal part mixture of both, are equivalent.

After having determined the most essential sample preparations parameters, which are extraction time and back extraction time and solvent, the sample preparation method was fully developed by determining the optimum extraction volume. The experimental data for this study is shown in Fig. 65. The back extraction volume was not optimized; instead, the volume used was $500 \mu \mathrm{L}$ because it is the smallest volume that covers most of the $5 \mathrm{~cm}^{2}$ FPSE media.

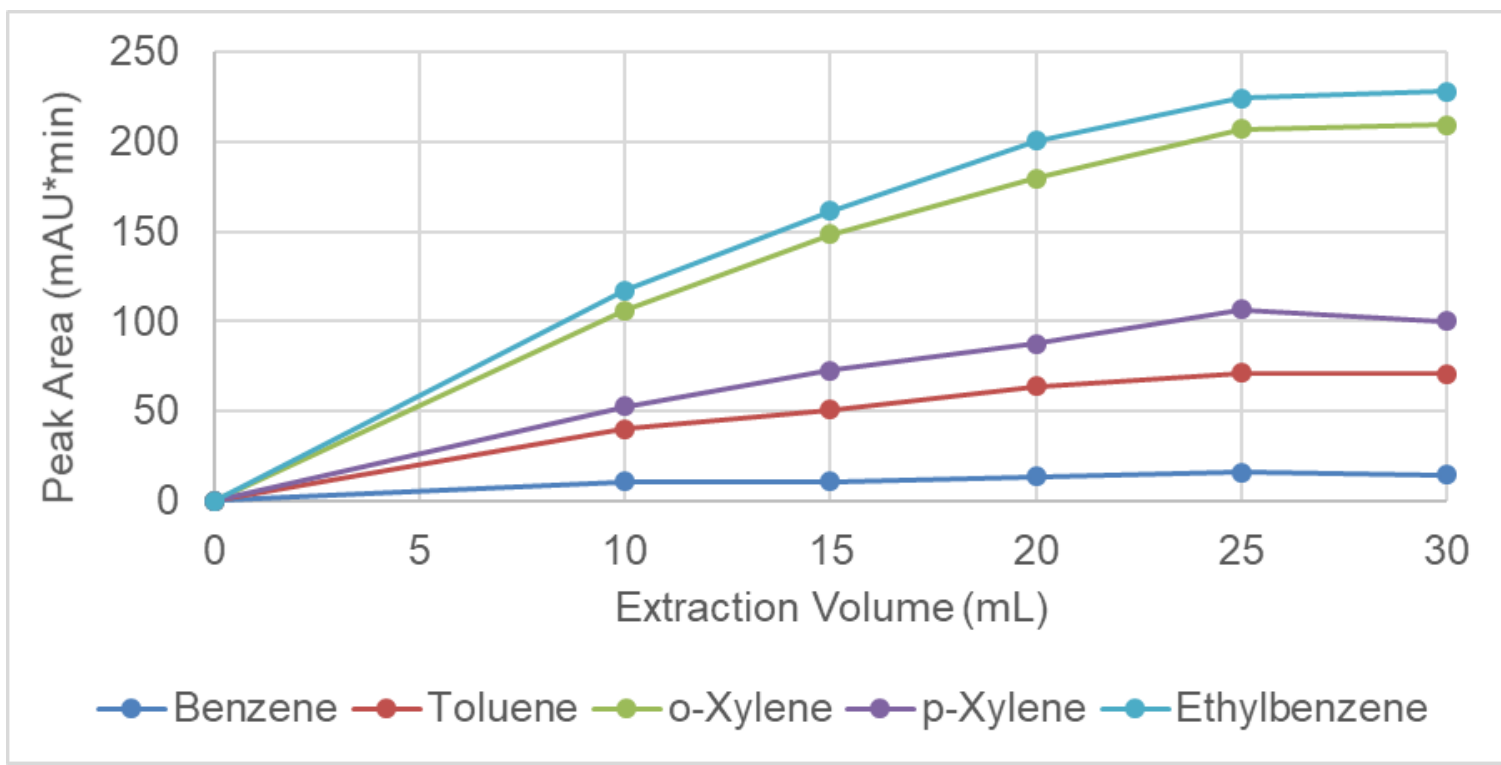

Figure 65: Experimental data of extraction volume optimization of the FPSE(PTHF)-HPLC-UV being developed for analysis of BTEX compounds in aqueous samples.

If the foremost goal of analysis is sensitivity and identification of compounds, the optimum extraction volume is $25 \mathrm{~mL}$, since there is no increase in the response of the analysis of $30 \mathrm{~mL}$ of solution vs $25 \mathrm{~mL}$. However, this conclusion is not 
compound specific. Benzene, for example, does not show any increase even after the lowest volume extracted from, which was $10 \mathrm{~mL}$. Additionally, it is important to always consider that for quantitation purposes the lowest volumes produce more accurate results because the partition coefficient is more favorable. A more favorable partition coefficient leads to an amount of analyte extracted that provides a better approximation of the concentration of said analyte in the sample. Therefore, in the main goal of the analysis is quantitation, and it often is, then the optimum extraction sample volume may be as low as 10 $\mathrm{mL}$ depending on sensitivity needs.

\subsubsection{Figures of Merit}

For this developed FPSE(PTHF)-HPLC-UV method, several figures of merit were determined, the first one being the dynamic range. Once again, the high end of the dynamic range was not explored thoroughly because it lacks any practical value. The highest concentration included in the linearity study is $10 \mathrm{ppm}$, which is very high, relatively speaking. This concentration of BTEX compounds would never be found in an environmental body of water or tap water, unless someone were to do it intentionally. Even so, an initial analysis would yield a very high signal and if it is beyond this confirmed dynamic range the sample could simply be diluted and a new analysis could be performed. The experimental data for the linearity study is found in Fig. 66. 


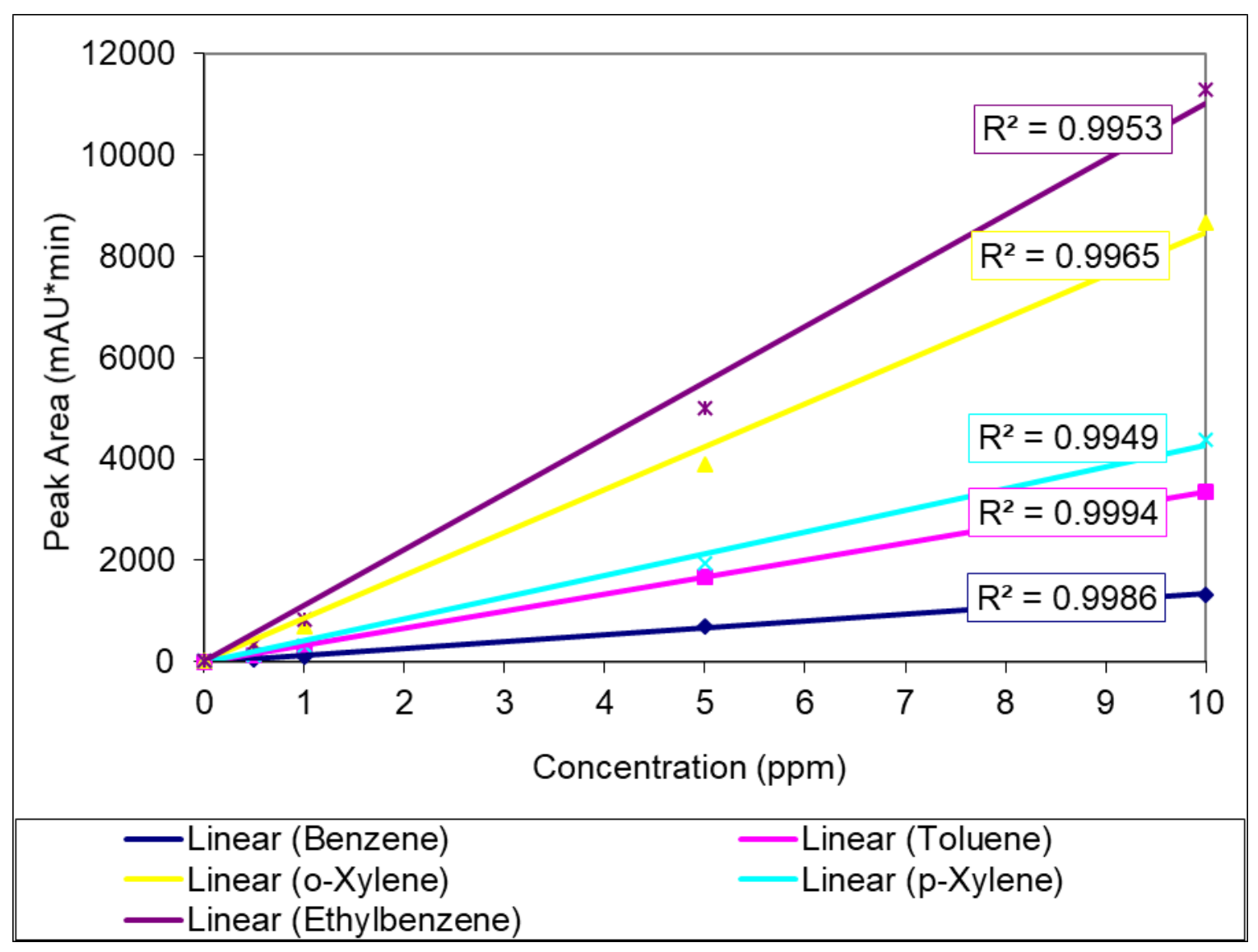

Figure 66: Experimental data of the linearity study for the developed FPSE(PTHF)-HPLC-UV method for analysis of BTEX compounds in aqueous samples.

After the dynamic range was determined the repeatability of the method was tested both intra $(n=5)$ and inter-day $(n=3)$. Inter-day, which was done in triplicate, is the more rigorous of the two kinds of repeatability studies but both are routinely reported because both are important. As shown in Table 8, the ranges of \% RSD values for intra and inter-day repeatability are $2.1-5.9$ and 3.0 -9.2 , respectively. These results are both satisfactory, well within accepted levels regularly published in highly regarded peer-reviewed scientific journals. In addition to the experimental results for repeatability, Table 8 includes a summary 
of the experimental data in Fig. 66, providing the determined dynamic range and corresponding correlation coefficient for each BTEX compound.

Table 8: Summary of the compound specific results for intra and inter-day repeatability as well as dynamic range and the corresponding correlation coefficient for the developed FPSE(PTHF)-HPLC-UV method for analysis of BTEX compounds in aqueous samples.

\begin{tabular}{|c|c|c|c|c|}
\multirow{2}{*}{ Compound } & \multicolumn{2}{c}{ Repeatability (\% RSD) } & \multicolumn{2}{c}{ Linearity } \\
\cline { 2 - 5 } & Intraday & Inter-day & Range $(\mathrm{ppb})$ & $\begin{array}{c}\text { Correlation } \\
\left(\mathrm{R}^{2}\right)\end{array}$ \\
\hline Benzene & 5.9 & 9.2 & $10-10000$ & 0.9986 \\
\hline Toluene & 2.4 & 6.5 & $10-10000$ & 0.9994 \\
\hline o-Xylene & 2.1 & 3.0 & $10-10000$ & 0.9965 \\
\hline p-Xylene & 3.6 & 5.5 & $10-10000$ & 0.9949 \\
\hline Ethylbenzene & 4.2 & 4.3 & $10-10000$ & 0.9953 \\
\hline
\end{tabular}

The last figures of merit to be obtained for this method were the limits of detection and quantitation. Table 9 includes the LOD and LOQ values for each BTEX compound calculated for the developed method and, for comparison purposes, LOD values for BTEX compounds published in 2014 by Gholivand et al. [140]. In this Analytica Chimica Acta article, the sample preparation was performed with custom made cobalt oxide $\left(\mathrm{Co}_{3} \mathrm{O}_{4}\right)$ nanoparticle traditional SPME fibers (platinum wire substrate) [140]. The extractions were made from the headspace. The instrumental analysis was done with GC-MS [140]. Headspace extractions followed by GC-MS analysis are suitable options for BTEX 
compounds because of their inherent low molecular mass, high stability and hydrophobicity that result in high volatility. If the compounds were not highly volatile headspace extractions would yield poor results because most would not be in the headspace to be extracted. In regards to $G C$ analysis, the high stability of the compounds eliminates the need for derivatization.

Table 9: Compound specific results for experimental calculations of LOD and LOQ for the developed FPSE(PEG)-HPLC-UV method and comparison to performance of a method in the literature.

\begin{tabular}{|c|c|c|c|}
\hline Compound & LOD (ppb) & LOQ (ppb) & $\begin{array}{c}\text { LOD (ppb) } \\
\text { Gholivand et al. }\end{array}$ \\
\hline Benzene & 1.5 & 5.1 & 11 \\
\hline Toluene & 0.7 & 2.4 & 7 \\
\hline o-Xylene & 0.6 & 2.0 & $1^{*}$ \\
\hline p-Xylene & 0.3 & 0.9 & $1^{*}$ \\
\hline Ethylbenzene & 0.3 & 0.9 & 6 \\
\hline
\end{tabular}

*The authors used a mixture of all 3 isomers of xylene (ortho, meta, para).

It is evident from the data in Table 9 that the novel FPSE(PTHF)-HPLC-UV method being introduced here provides much better sensitivity than the $\operatorname{SPME}\left(\mathrm{Co}_{3} \mathrm{O}_{4}\right.$ nanoparticles)-GC-MS method found in the literature.

\subsubsection{Application to Real Samples}

The developed and validated FPSE based method was used on three different kinds of aqueous samples: tap water, reclaimed water, and environmental water from a pond at FIU's Modesto Maidique Campus. An aliquot of each of the 
samples was analyzed for the presence of BTEX compounds but none were found. The remaining volume was then spiked to a concentration of $10 \mathrm{ppb}$ per compound and analyzed with the developed method. None of the samples were pretreated in any way before extraction. For comparison purposes a standard solution (deionized water as the solvent) of the same concentration of the BTEX compounds was also analyzed. The experimental data is portrayed in Fig. 67.

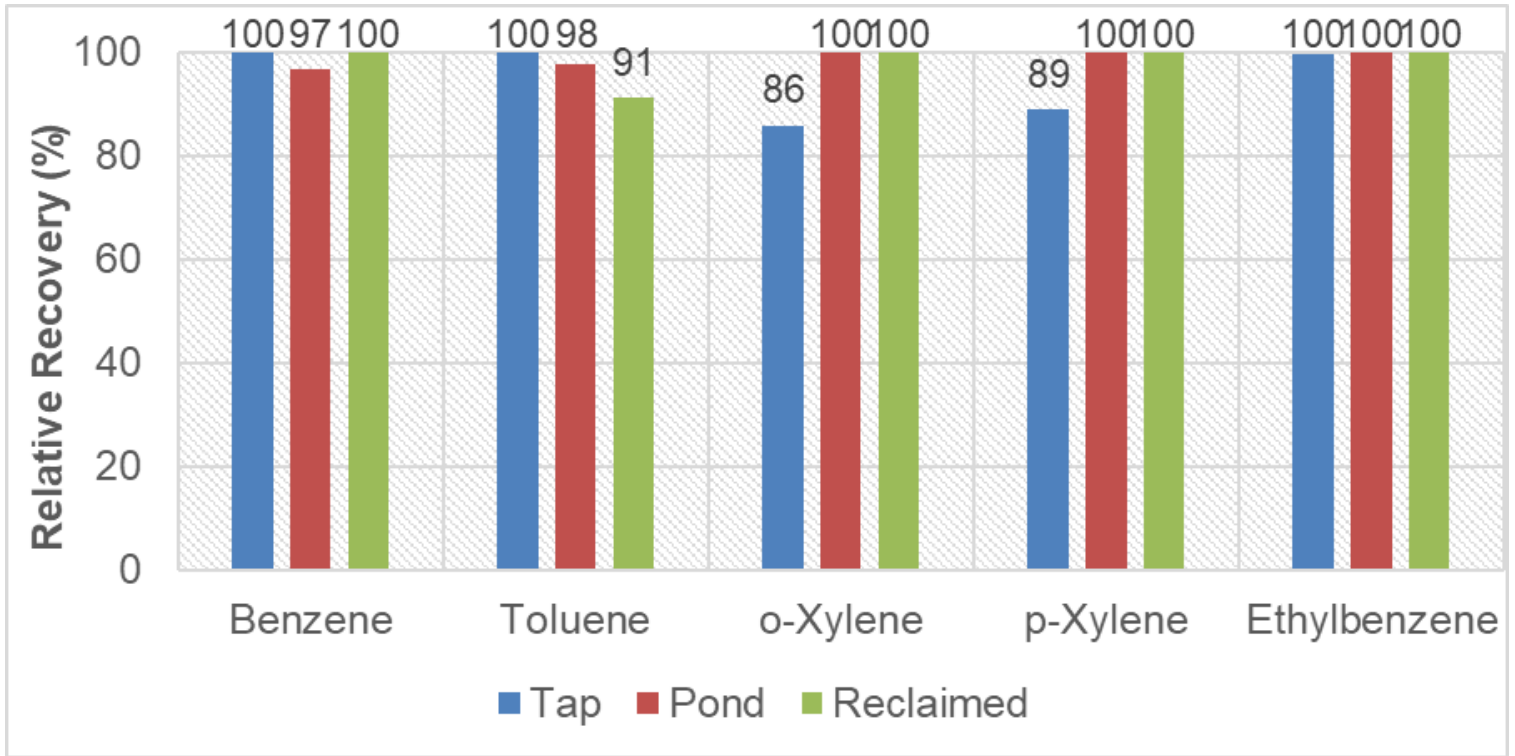

Figure 67: Experimental data showing results of the application of the developed FPSE(PEG)-HPLC-UV method to spiked samples of tap, pond, and reclaimed water in the form of relative recovery \%. The samples were spiked to a concentration of $10 \mathrm{ppb}$ per analyte.

The developed method is clearly very effective for analysis of these important and challenging sample matrices. The relative recovery is at or near $100 \%$ for each analyte from these samples, so the obtained sensitivity levels given in the form of LOD and LOQ values can also be achieved when using these methods for analysis of tap water, reclaimed water, and environmental fresh water samples. 


\subsection{Substituted Phenols}

Substituted phenols are very dangerous to humans due to their toxic nature $[9,10]$. Compounds in this class bind to hemoglobin [9] and affect several body systems, including the central nervous system, of human beings depending on the amount of exposure and how prolonged that exposure is [11]. Wood processing plants, pharmaceutical labs, and oil refineries are all enterprises that commonly use phenol or substituted phenols and as such their waste disposal practices should be closely monitored and inspected $[9,10]$. It would be grave criminal behavior for any company to not follow safe disposal procedures for chemicals such as these. Another process that can produce methyl substituted phenols as by-products is the degradation of the organic portion within the composition of soil [9]. The following FPSE-HPLC-UV method, developed and validated for 5 substituted phenols (listed in Table 4), is intended to serve as a reliable option to conduct ultra-trace analysis of aqueous matrices for any compound within this classification.

\subsubsection{Preparation of Standard Solutions}

Primary stock solutions of the individual substituted phenols (4-chlorophenol; 3,5dimethylphenol; 2,6-dichlorophenol; 2,4,6-trichlorophenol; 2,4-diisopropylphenol) included in this research, which were purchased in bulk as pure liquids, were prepared with methanol as the solvent. The concentration of each of these highly concentrated stock solutions was 10,000 ppm. From these standards an intermediate solution was made, again with methanol as the solvent. This intermediate was a mixture of all five substituted phenols where the 
concentration of each was $100 \mathrm{ppm}$. This intermediate solution was used whenever necessary to make other solutions in methanol for instrumental method development or to spike deionized waters or other aqueous matrices for FPSE method development or validation. The methanol used as the solvent was always HPLC grade, for this and all projects presented herein.

\subsubsection{Instrumental Method Development}

The development of the instrumental method, as is always the case, had to be done before the FPSE method could be developed. Doing this with an HPLC-UV equipped with a C-18 Extend analytical column was rather trivial. First, each compound was injected individually at a high concentration (10 ppm) to make sure that they could be detected and to confirm the order of elution on the C-18 column. The injection of the compounds individually served these purposes and provided a starting point from which to perfect the method. These first injections were made with a standard isocratic method which consisted of a $1 \mathrm{~mL} / \mathrm{min}$ flow rate, 50:50 water:acetonitrile mobile phase composition, $5 \mu \mathrm{L}$ injection volume, and the wavelength of the UV detector set at $200 \mathrm{~nm}$. After this the only parameter that was changed was the mobile phase composition, which ultimately became 69:41 water:acetonitrile and provided satisfactory performance assessed primarily on the resolution. Fig. 68 shows a representative chromatogram obtained with this instrumental method. 


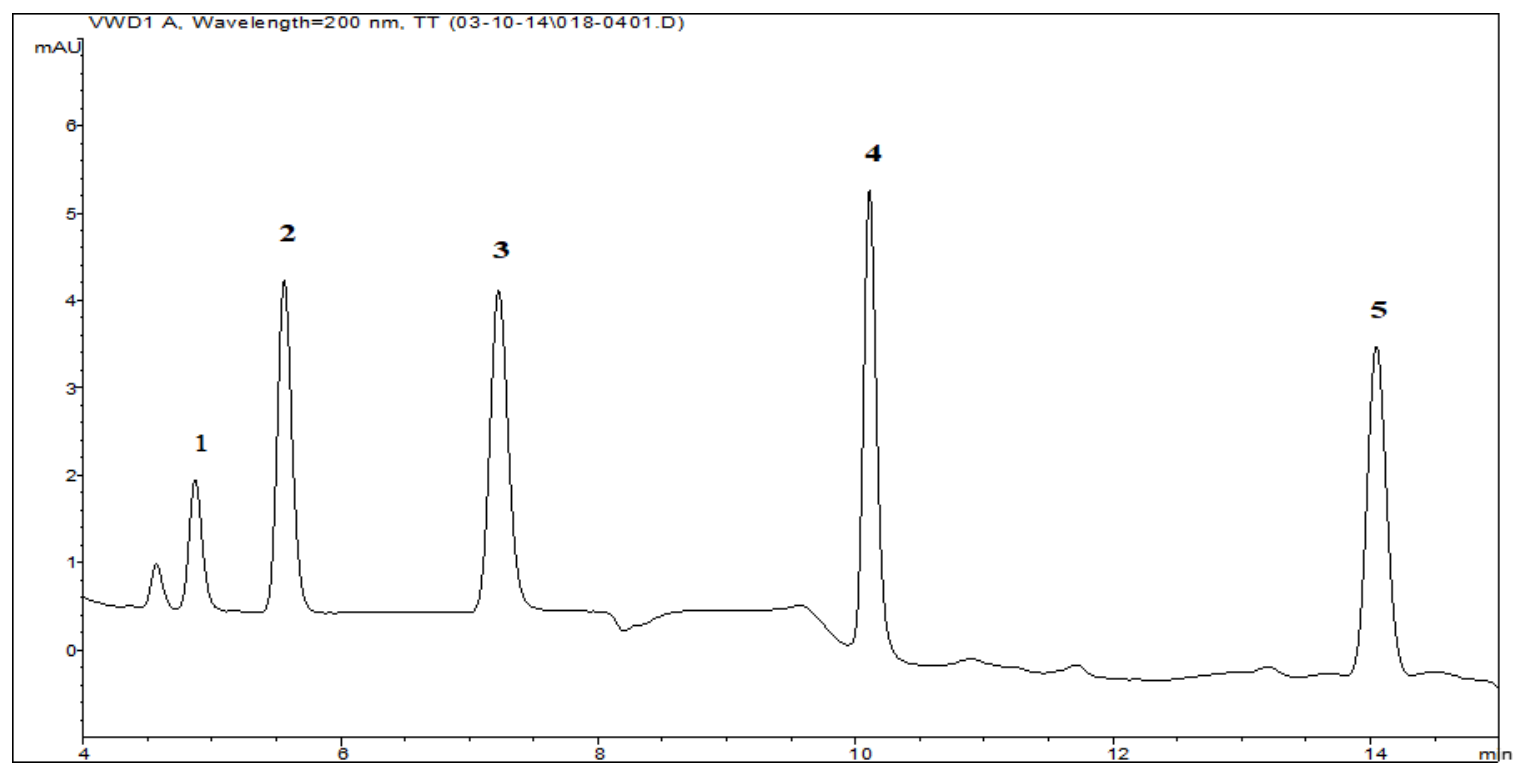

Figure 68: Representative HPLC-UV chromatogram of an analysis of 5 substituted phenols from deionized water. The elution order from the $\mathrm{C}-18$ Extend column is as follows: (1) 4-chlorophenol, (2) 3,5-dimethyl phenol, (3) 2,6dichlorophenol, (4) 2,4,6-trichlorophenol, (5) 2,4-diisopropyl phenol.

\subsubsection{Determination of Optimum FPSE Media Sorbent}

The optimum FPSE media for extraction of substituted phenols from water was determined by comparison three different sol-gel sorbents with sets of triplicate extractions from $10 \mathrm{~mL}$ aliquots of the same $100 \mathrm{ppb}$ standard solution in deionized water. The active components (organic polymer in the organicinorganic hybrid sol-gel sorbents) tested were PEG, PTHF, and a combination of PEG and polyethylene oxide (PEO). PEG and PEO are practically identical polymers, but the term PEO is used when the molar mass of the polymer is more than $100,000 \mathrm{~g} / \mathrm{mol}$. These FPSE media were chosen because of their polar nature, which is necessary for the extraction of polar molecules from aqueous matrices. The best performance was obtained with the FPSE(PEG) media, as seen in Fig. 69. 


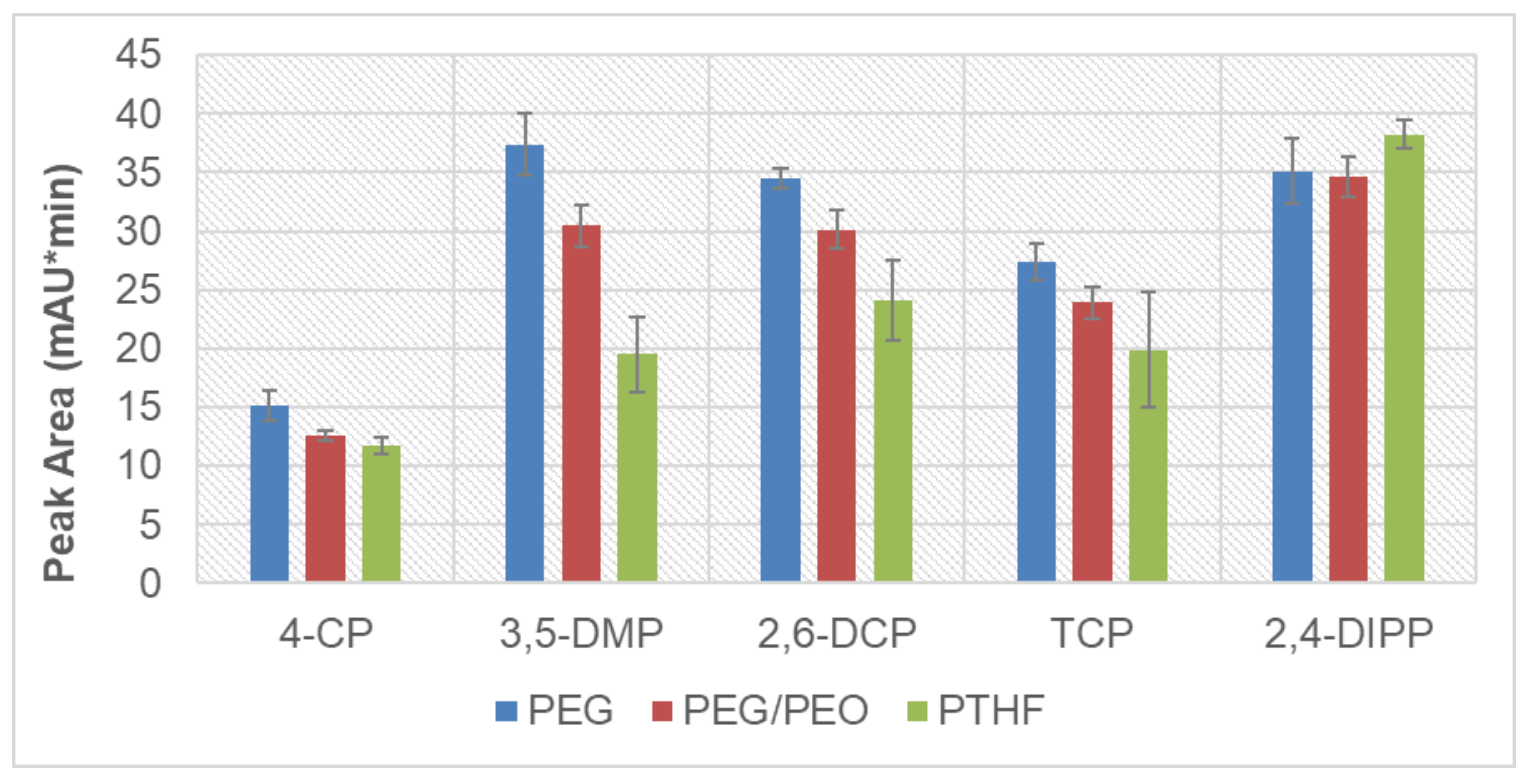

Figure 69: Experimental data of the comparison of the effectiveness of FPSEPTHF, FPSE-PEG, and FPSE-PEG/PEO media, respectively, for extraction of substituted phenols from aqueous matrices.

\subsubsection{Optimization of Sample Preparation Parameters}

Once it was determined that the best available FPSE media for extractions of substituted phenols from water were the ones with sol-gel PEG sorbent as the organic component, the sample preparation parameters were optimized, starting with the extraction time. Triplicate extractions were made from standard solutions in deionized water, the only difference among the sets of extractions being the time allowed for extraction. The results are shown in Fig. 70. 


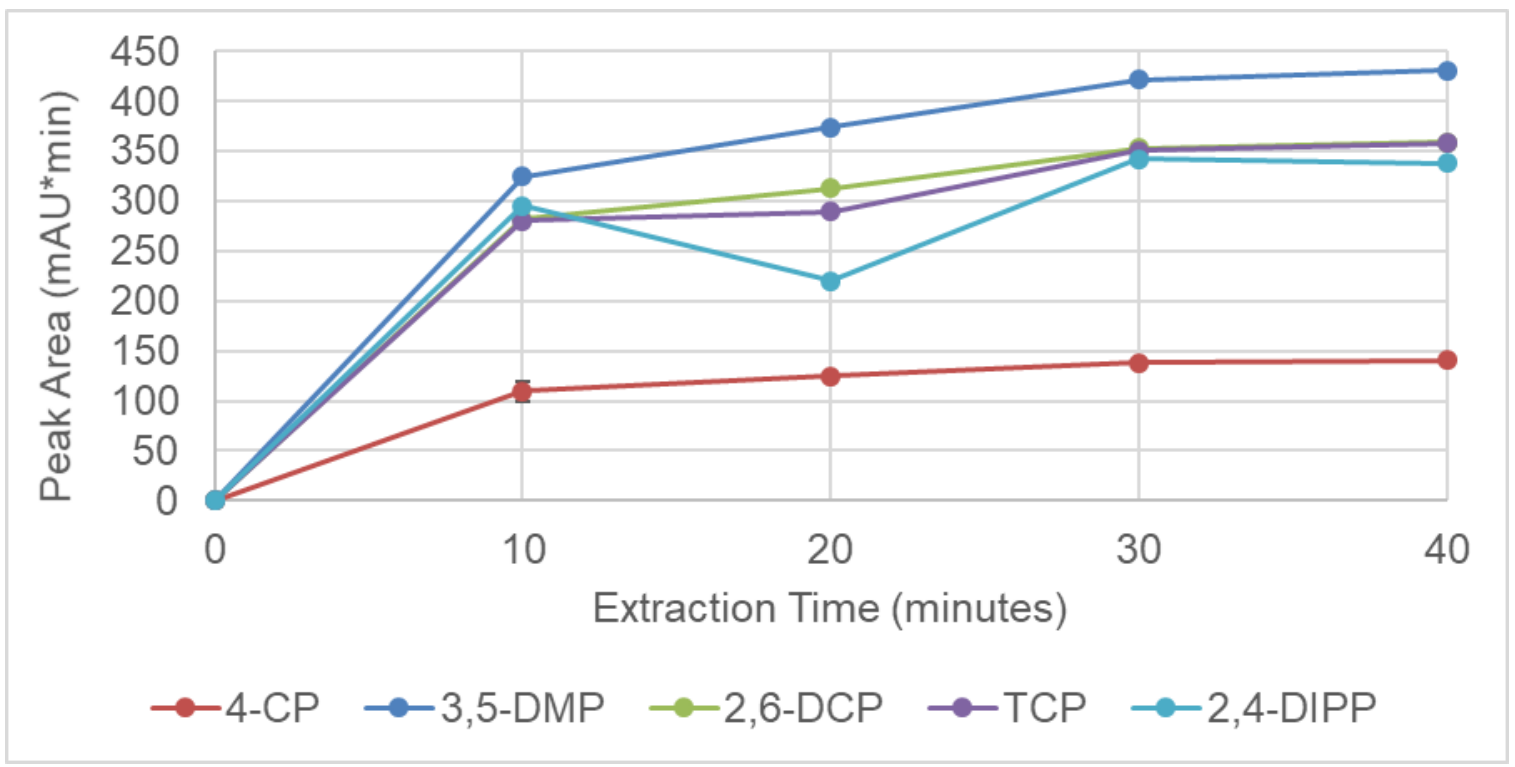

Figure 70: Experimental data of extraction time optimization of the FPSE(PEG)HPLC-UV method being developed for analysis of substituted phenols in aqueous samples.

The optimum extraction time, with sensitivity being the foremost consideration, was chosen to be 30 minutes. As is usually the case in sample preparation method development, this is not necessarily the best choice for any criteria. In a situation where the minimum concentration threshold is relatively high when considering the highly sensitive nature of FPSE media, the optimum extraction time would be 10 minutes or even shorter.

The back extraction time was determined in the same manner. This process is inherently much faster than extraction. As can be deduced from the experimental data presented in Fig. 71, there is no need to back extract substituted phenols from FPSE-PEG media for longer than 5 minutes. The is a seemingly significant increase in response magnitude for 2,4-DIPP from 5 minute back extraction to 10 
minute back extraction, but it is not the case for the other four substituted phenols.

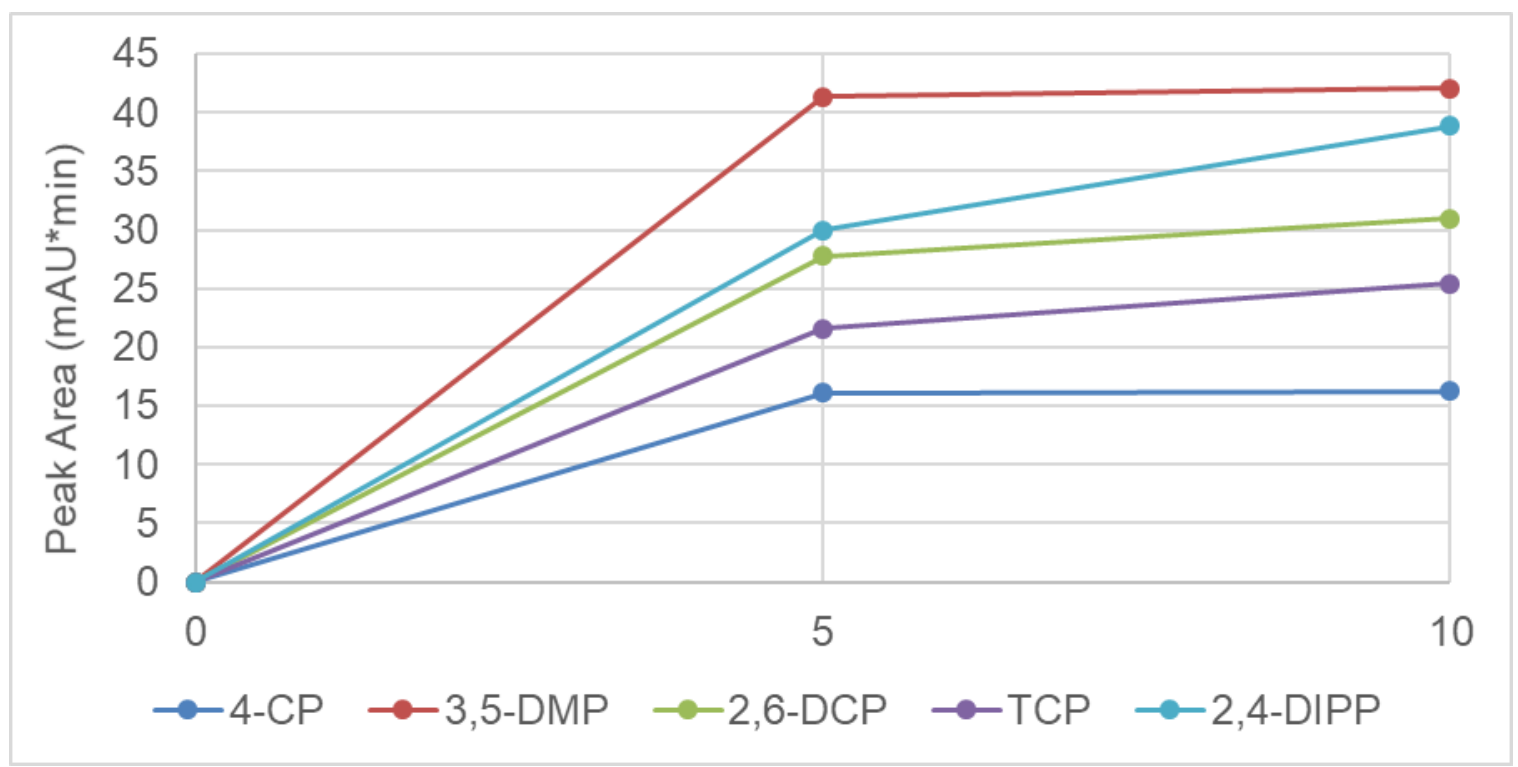

Figure 71: Experimental data of back extraction time optimization of the FPSE(PEG)-HPLC-UV method being developed for analysis of substituted phenols in aqueous samples.

Another back extraction related optimization was carried out, this time for the identity of the solvent that would be used. A comparison was carried out testing the efficiencies of $\mathrm{MeOH}, \mathrm{ACN}$, and an equal part mixture of the two. This was done in triplicate using the parameters optimized to this point. The resulting experimental data showed no significant difference among the three options, and ACN was used for the rest of the method development and then validation.

The last parameter optimized for this method was the extraction sample volume. This is done by doing triplicate sets of extractions with the method as optimized to this point. The analysis was of aliquots of the same standard solution of the substituted phenols in deionized water, the only difference among the sets of 
extractions being the volume from which is extracted. As discussed previously, this experiment in the method development process tends to produce interesting results because while increasing the volume can yield higher responses, it also significantly drops the relative recovery and therefore the efficiency and accuracy of quantitation of the method. The results for these compounds in deionized water are shown in Fig. 72:

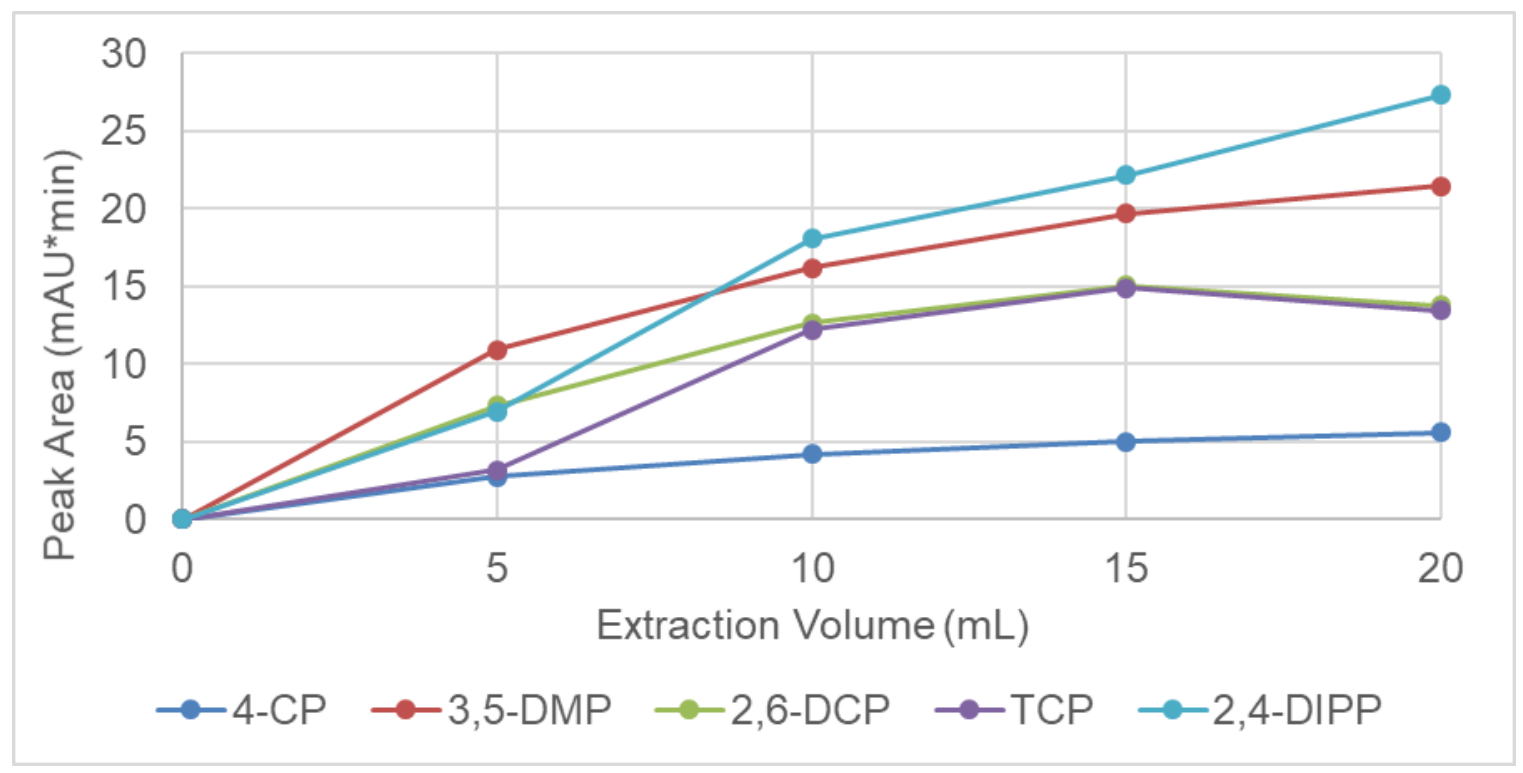

Figure 72: Experimental data of extraction sample volume optimization of the FPSE(PEG)-HPLC-UV method being developed for analysis of substituted phenols in aqueous samples.

In this case the study was not continued beyond $20 \mathrm{~mL}$ of sample because at this point, when compared to the results for $15 \mathrm{~mL}$, it is evident that a plateau has been reached. The responses for 2,4-DIPP and 3,5-DMP trend upward from 15 
to $20 \mathrm{~mL}$ but the ones for 2,6-DCP and TCP trend downward, which leads to the conclusion that the overall amount extracted is not increasing. While $15 \mathrm{~mL}$ is the optimum extraction sample volume for sensitivity purposes, $10 \mathrm{~mL}$ would be the choice for quantitative analysis. It is not that much less sensitive than $15 \mathrm{~mL}$ while providing better accuracy of quantitation.

\subsubsection{Figures of Merit}

It is well known that sol-gel materials offer great stability, including the chemical and solvent variety. FPSE is a recent creation, however, and as such it was considered necessary to test this characteristic of sol-gel materials by testing FPSE(PEG) media. Triplicate analyses of substituted phenols from deionized water were performed using the developed method, then these FPSE media were exposed to acetone for eight hours. After this exposure to acetone, the FPSE media were used again for extraction from the same solution from before the exposure to the acetone. There was no significant difference in the results of the analyses before and after exposure to acetone for eight hours. The same procedure was also used to test the stability of the FPSE-PEG media in methylene chloride, $0.1 \mathrm{M} \mathrm{HCL}$ solution $(\mathrm{pH}=1)$, and $0.1 \mathrm{M} \mathrm{NaOH}$ solution $(\mathrm{pH}=$ 13), respectively. The effectiveness of the FPSE-PEG media was not affected by this prolonged exposure to these four different chemical environments, thereby confirming the stability related prowess of sol-gel materials applies when used as FPSE sorbents. 
The dynamic range of the developed FPSE(PEG)-HPLC-UV method was tested for each substituted phenol. The solutions were all made by diluting the solution of the next higher concentration. From each solution $10 \mathrm{~mL}$ aliquots were obtained and from each aliquot the substituted phenols were extracted. Other than the concentrations there were not any differences among the sets of extractions and they were all performed simultaneously. The results of this investigation are shown in Fig. 73. The range could easily have been extended above $5000 \mathrm{ppb}$ but this would not serve any practical purpose because concentrations would never be that high in a real sample.

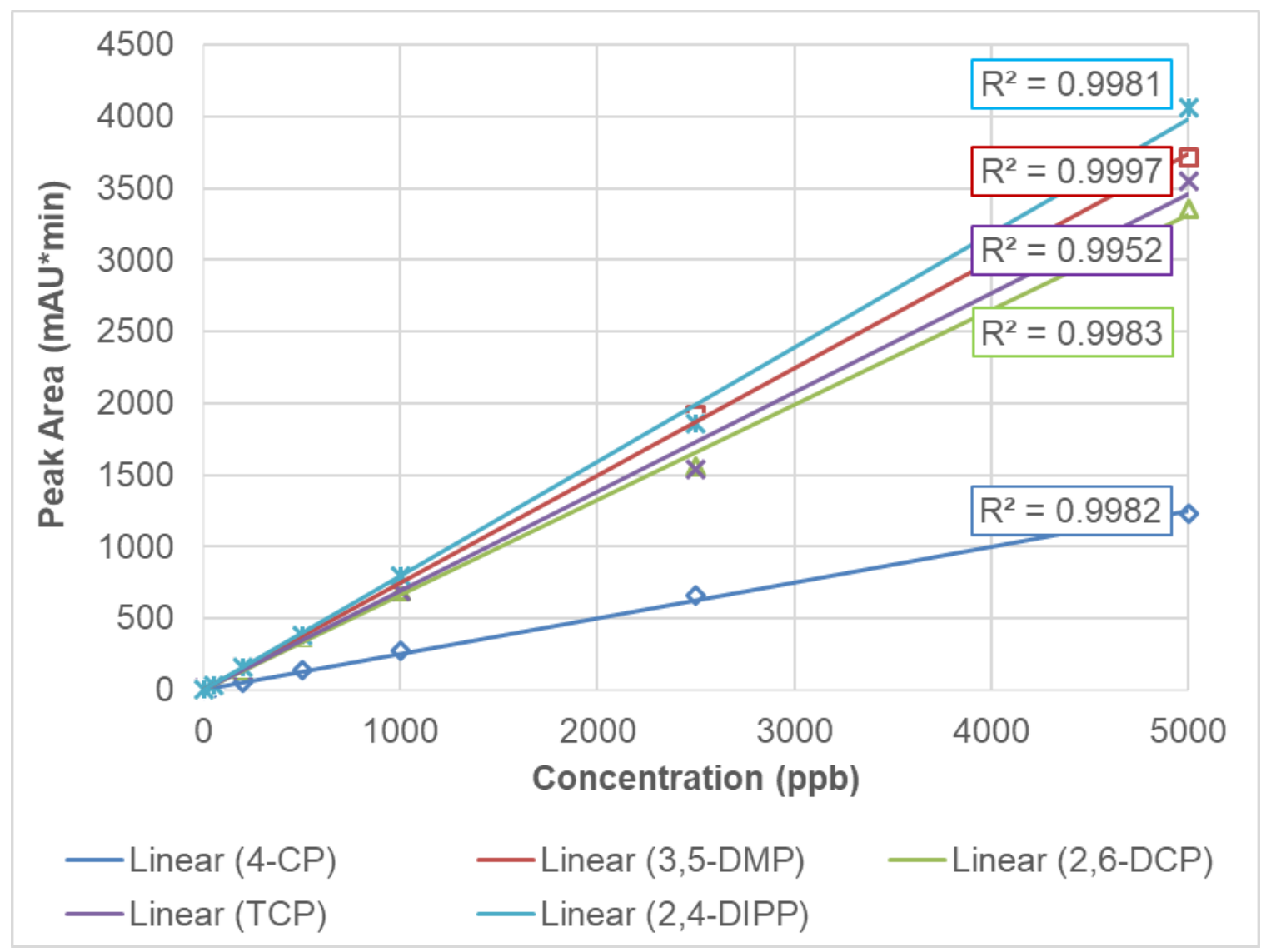

Figure 73: Experimental data of linearity study of the FPSE(PEG)-HPLC-UV method developed for analysis of substituted phenols in aqueous samples. 
These linear trendlines, in Fig. 73, which include points at 1, 50, 200, 500, 1000, 2500 , and $5000 \mathrm{ppb}$, show superb correlation $\left(R^{2}>0.9950\right)$ between these concentrations and their corresponding responses for each substituted phenol in the study.

The next part of the method validation was determination of repeatability intra and inter-day. For the intraday study, 5 extractions from the same solution of the 5 substituted phenols in this research project were spread out throughout one day. For the responses from those 5 extractions and subsequent analysis via HPLC-UV the corresponding \%RSD value was calculated for each compound. The inter-day study was done in a similar fashion but this time the closeness of the responses was determined over three days instead of 5 extractions within one day. Once again the \%RSD for the compound specific responses was determined. A summary of the results for the linearity study and the \%RSD values from both repeatability studies are presented for each substituted phenol in Table 10. The values in Table 10 are strong evidence of the viability of this method in water quality laboratories, with a large dynamic range that exhibits outstanding correlation values and repeatability that is well within acceptable limits both within one day and over several days. 
Table 10: Summary of the compound specific results for intra and inter-day repeatability as well as dynamic range and the corresponding correlation coefficient for the developed FPSE(PEG)-HPLC-UV method for analysis of substituted phenols in aqueous samples.

\begin{tabular}{|c|c|c|c|c|}
\multirow{2}{*}{ Compound } & \multicolumn{2}{c}{ Repeatability (\% RSD) } & \multicolumn{2}{c}{ Linearity } \\
\cline { 2 - 5 } & Intraday & Inter-day & Range $(\mathrm{ppb})$ & $\begin{array}{c}\text { Correlation } \\
\left(\mathrm{R}^{2}\right)\end{array}$ \\
\hline 4-CP & 1.8 & 25.5 & $1-5000$ & 0.9981 \\
\hline 3,5-DMP & 1.6 & 9.8 & $1-5000$ & 0.9997 \\
\hline 2,6-DCP & 1.3 & 0.5 & $1-5000$ & 0.9983 \\
\hline TCP & 1.6 & 6.0 & $1-5000$ & 0.9952 \\
\hline 2,4-DIPP & 5.1 & 4.9 & $1-5000$ & 0.9982 \\
\hline
\end{tabular}

After determination of the dynamic range for each compound and the repeatability of this method, the next figures of merit that needed to be determined were the limits of detection and quantitation. Table 11 includes the LOD and LOQ values calculated for each substituted phenol and LOD values for three of these compounds published in 2005 by Bagheri et al. [141]. This Analytica Chimica Acta article presented a HS-SPME(polyaniline)-GC-FID method for analysis of phenolic compounds from aqueous matrices [141]. Headspace extractions followed by GC-based analysis are a curious choice for the analysis of phenols, especially without derivatization. As discussed previously, compounds that are most suitable for headspace analysis, especially when they need to be driven out of water, are those that are very stable and hydrophobic. Substituted phenols are weakly acidic and therefore do not remain 
protonated without derivatization. These compounds are also not hydrophobic, which makes it difficult to get a vast majority of them out of the aqueous sample matrix and into the headspace. It is likely that the performance of this method suffered because of the decision to extract from the headspace in addition to the several well-documented deficiencies of SPME fibers. In regards to GC analysis, derivatization of acidic compounds is the norm.

Table 11: Compound specific results for experimental calculations of LOD and LOQ for the developed FPSE(PEG)-HPLC-UV method and comparison performance of a method in the literature.

\begin{tabular}{|c|c|c|c|}
\hline Compound & LOD (ppb) & LOQ (ppb) & $\begin{array}{c}\text { LOD (ppb) } \\
\text { Bagheri et al. }\end{array}$ \\
\hline 4-CP & 0.03 & 0.10 & 58 \\
\hline 3,5-DMP & 0.01 & 0.04 & $\mathrm{~N} / \mathrm{A}$ \\
\hline 2,6-DCP & 0.04 & 0.13 & $3.7^{*}$ \\
\hline TCP & 0.02 & 0.08 & 1.3 \\
\hline 2,4-DIPP & 0.02 & 0.07 & $\mathrm{~N} / \mathrm{A}$ \\
\hline
\end{tabular}

${ }^{*}$ This value corresponds to 2,4 -DCP instead. It is reasonable to expect the value of this method for 2,6-DCP to be comparable to that of 2,4-DCP.

It is evident from the data in Table 11 that the novel FPSE(PEG)-HPLC-UV method being introduced here provides much better sensitivity than the HSSPME(polyaniline)-GC-FID published by Bagheri et al. Additionally, this FPSEbased method outperforms a headspace HS-SPME(polyacrylate)-HPLC-UV method published in 2002 by Penalver et al. for the sensitivity of TCP, which 
obtained an LOD value for that compound of 2.0. The other substituted phenols included here were not included in that study.

\subsubsection{Field Application to Tap, Pond, and Reclaimed Water Samples}

The developed and validated FPSE(PEG)-HPLC-UV method was applied to analysis of substituted phenols from tap, pond, and reclaimed water samples and the effectiveness of said analyses was compared with that of analysis of deionized water spiked to the same concentration (10 ppb). Each sample was first extracted from without any pretreatment or spiking. None of the five substituted phenols for which the method was developed were found in these samples. After spiking, all the samples were analyzed simultaneously under the same conditions. The aliquots extracted from were $10 \mathrm{~mL}$ in volume. The results of this comparison are shown in Fig. 74 .

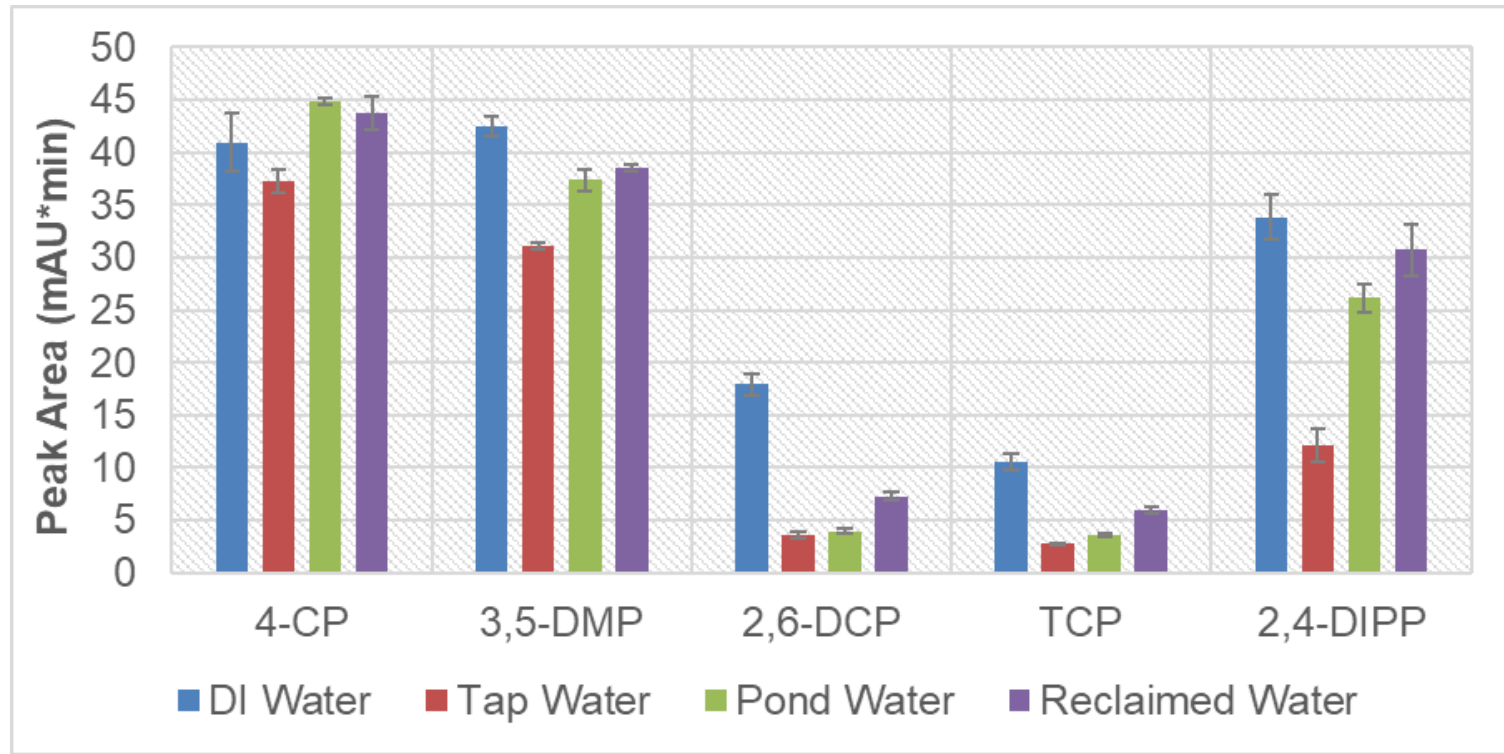

Figure 74: Comparison of response magnitudes and triplicate set repeatability for analysis of deionized, tap, pond, and reclaimed water samples spiked with five substituted phenols. 
The percent recovered of substituted phenols in tap, pond, and reclaimed water samples were in the range of $19.7-91.0,21.9-100$, and $40.7-100$, respectively. These results are not ideal, but certainly interesting and lead to questions that can only be answered with further research. What it is in these samples and what is interacting with the substituted phenols? Why is the relative recovery of some analytes such as 2,6-DCP and TCP much lower than that of others?

Application of FPSE based methods to environmental samples for analysis of other analytes such as nitroaromatic explosives and BTEX compounds has resulted in much better performance when compared to analysis of deionized water. This indicates that the issue is, in fact, complexing of the substituted phenols with other compounds or particulates in these water samples. If this is the case, the substituted phenols were extracted from tap, pond, and reclaimed water but not as individual compounds and as such were not detected by the HPLC-UV method.

\subsubsection{FPSE(PEG)-GC-MS}

One of the many desirable features of FPSE as a sample preparation technology is that it can seamlessly be coupled to just about any kind of analytical instrument. This ability of FPSE is illustrated in Fig. 75, which shows a gas chromatography mass spectrometry (GC-MS) chromatogram of an FPSE(PEG)GC-MS analysis of the five pertinent substituted phenols in deionized water (50 $\mathrm{ppb}$ concentration of each analyte). After back extraction of the analytes from the FPSE media, following the exact procedure for HPLC analysis, the substituted 
phenols were introduced to the GC-MS by way of $1 \mu \mathrm{L}$ splitless liquid injection. The peaks for the first three compounds (3,5-dimethyl phenol, 4-chlorophenol, and 2,6-dichlorophenol, respectively) exhibit a significant peak tail; this is likely due to hydrogen ion loss by some of the molecules. The peak tailing could have been avoiding with derivatization but this was not performed because the purpose of this GC-MS injection was simply to prove that the same FPSE(PEG) sample preparation procedure can be used for both kinds of instruments. GCs and HPLCs are not only very different fundamentally and practically, these are also the most common chromatographic instruments used presently.

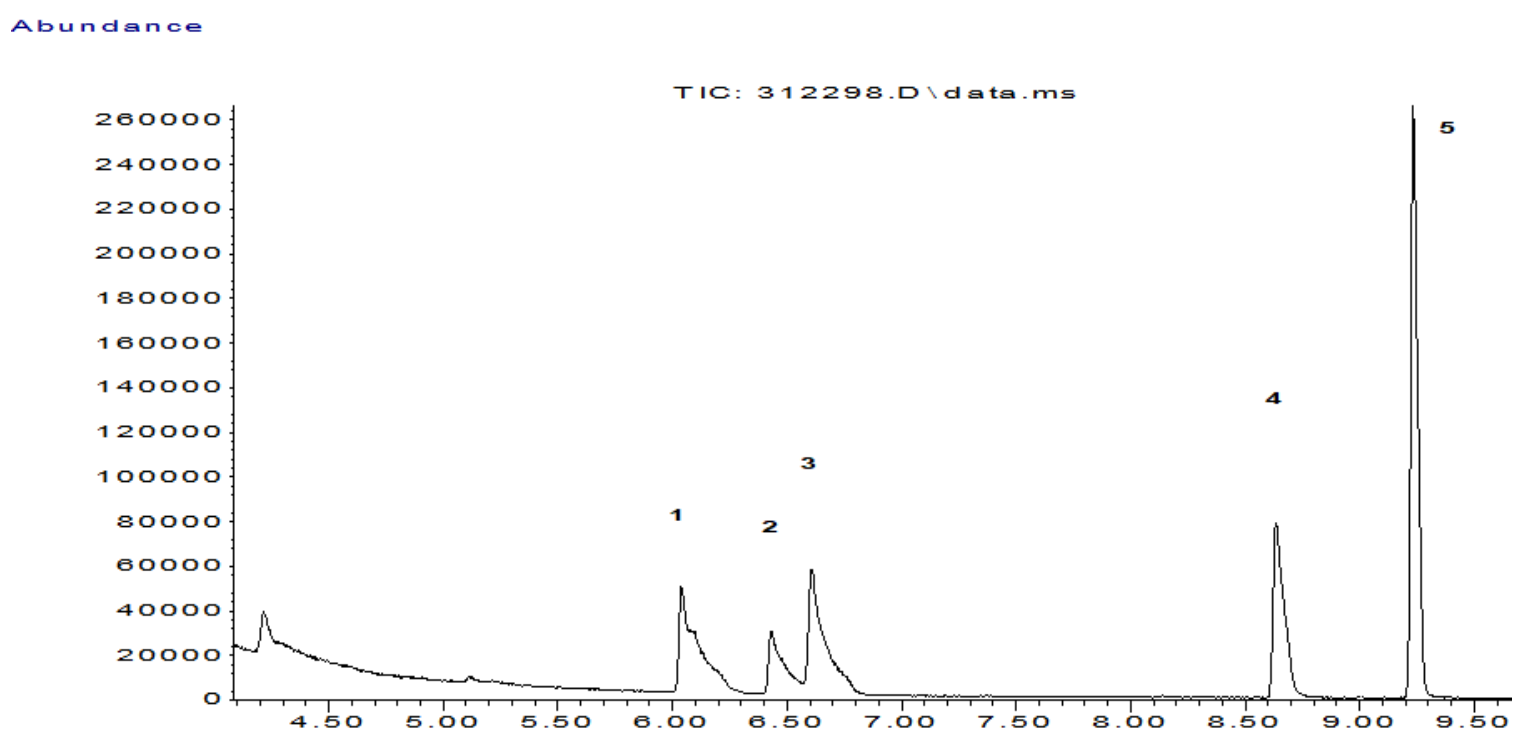

Figure 75: Representative GC-MS chromatogram of an analysis of 5 substituted phenols from deionized water. The elution order from the GC column is as follows: (1) 3,5-dimethyl phenol, (2) 4-chlorophenol, (3) 2,6-dichlorophenol, (4) 2,4,6-trichlorophenol, (5) 2,4-diisopropyl phenol.

\subsection{Polycyclic Aromatic Hydrocarbons}

PAHs are a very well known, infamous even, class of pollutants compounds; 16 are considered priority pollutants by the US EPA because they are known 
carcinogens and pose a grave threat to the health of humans and ecosystems $[108,109]$. In addition to being a priority for the US EPA, PAHs are recognized as the second most abundant type of environmental pollutant in China [110]. Considering that PAHs are priority pollutants because of their carcinogenic properties [111], it is quite worrisome that their existence is so prevalent and growing. Since compounds result from combustion processes, their existence in the environment will become more prevalent over time, not less. An FPSE based method for simultaneous analysis of 5 PAHs (listed in Table 3) that can potentially become the regulatory method for monitoring of these compounds in the environment has been developed and validated because having the ability to accurately measure their concentrations in environmental waters and other samples is very important [109].

\subsubsection{Preparation of Standard Solutions}

Each polycyclic aromatic hydrocarbon included in this research was purchased in bulk and dissolved in acetonitrile individually, the concentration of each standard stock solution being $10,000 \mathrm{ppm}$. These solutions were diluted individually to 1 ppm for instrumental method development. A $10 \mathrm{ppm}$ intermediate of all 5 compounds together was used for spiking of deionized, pond, tap, or reclaimed water samples for method development, validation, or application. Dilutions were always planned to require the fewest steps possible to reduce error. 


\subsubsection{Determination of Optimum FPSE Media Sorbent-}

PAHs are nonpolar, hydrophobic analytes that lend themselves well to be extracted from aqueous solutions. Despite their lack of polarity, FPSE media were tested for their extractions that exhibit a wide range of polarity in their solgel sorbent: sol-gel PEG (highly polar), sol-gel PTHF (mildly polar), PDMDPS (nonpolar with phenyl functionality). Having covered this range of polarities by FPSE media presents a complete picture of how extraction efficiency is affected by this characteristic of sorbents. The resulting data clearly indicates that the best of these three FPSE media for analysis of PAHs is PDMDPS. This is what was expected because PDMDPS is nonpolar and provides phenyl functionality that contributes to fast and lasting retention of PAHs via pi-pi interactions. From this point on only FPSE-PDMDPS media were used for analysis of PAHs.

\subsubsection{Instrumental Method Development}

Individual injections of each PAH (1 ppm were made into the HPLC-UV to confirm the order of dilution by the selected C-18 Extend column with an initial generic method in which the mobile phase composition was 50:50 $\mathrm{H}_{2} \mathrm{O}: \mathrm{ACN}$ and the flow rate was $1 \mathrm{~mL} / \mathrm{min}$. This confirmed the expectation (based on methods in the literature) that the order of elution is naphthalene, fluorene, anthracene, phenanthrene and fluoranthene. With this preliminary as a starting point, a much better one was developed such that there is baseline separation one either side of each peak $\left(R_{s} \geq 1.5\right)$ while making the method as time efficient as possible. The method parameters are the following: isocratic with $1 \mathrm{~mL} / \mathrm{min}$ of $30: 70$ $\mathrm{H}_{2} \mathrm{O}: \mathrm{ACN}$ mobile phase composition; $30^{\circ} \mathrm{C}$ column compartment controlled 
temperature; detector set at $217 \mathrm{~nm}$ from $0-6$ minutes, $254 \mathrm{~nm}$ from $6-9$ minutes, and $217 \mathrm{~nm}$ from $9-10.5$ minutes. A representative chromatogram of the resulting method is presented in Fig. 76. This chromatogram corresponds to the resulting back extraction solution of a FPSE(PDMDPS)-HPLC-UV analysis of the 5 pertinent PAHs from deionized water.

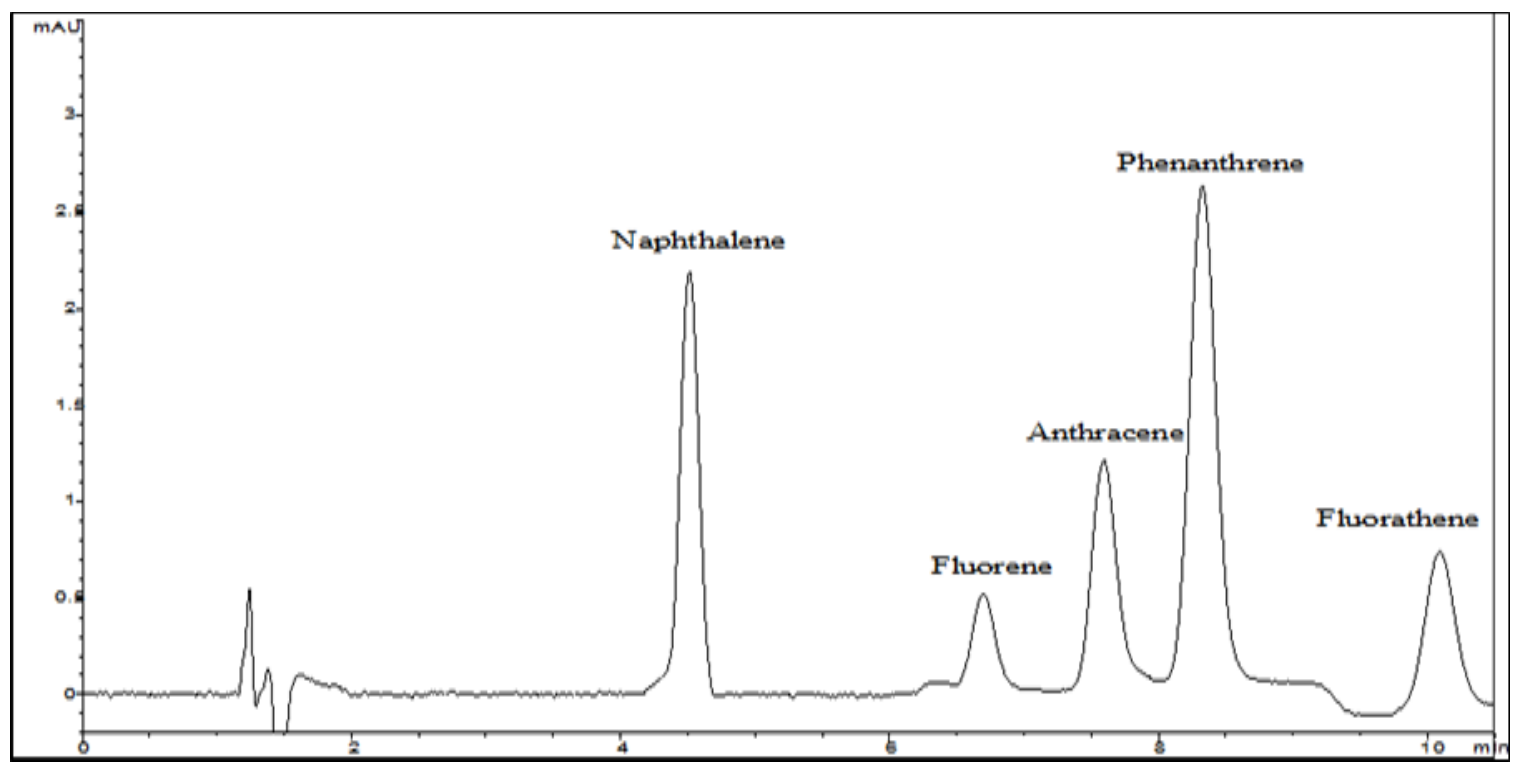

Figure 76: Representative HPLC-UV chromatogram of an analysis of 5 PAHs from deionized water. Each peak is labeled with the identity of the corresponding compound.

\subsubsection{Optimization of Sample Preparation Parameters}

The extraction time optimization for analysis of PAHs in deionized water was relatively short, as expected, given that these compounds are hydrophobic and the FPSE-PDMDPS media are very receptive to them because of similar polarity and also pi-pi interactions. Only three sets of triplicate extractions were required, all easily performed simultaneously. The entire process, including extractions, back extractions and HPLC-UV analysis only require approximately 90 minutes 
of real time. The experimental results of this study, shown in Fig. 77 , show that there is no increase in response after 20 minutes of extraction time. This makes 20 minutes the optimum extraction time for sensitivity. However, 10 minutes provides great sensitivity as well with much better efficiency.

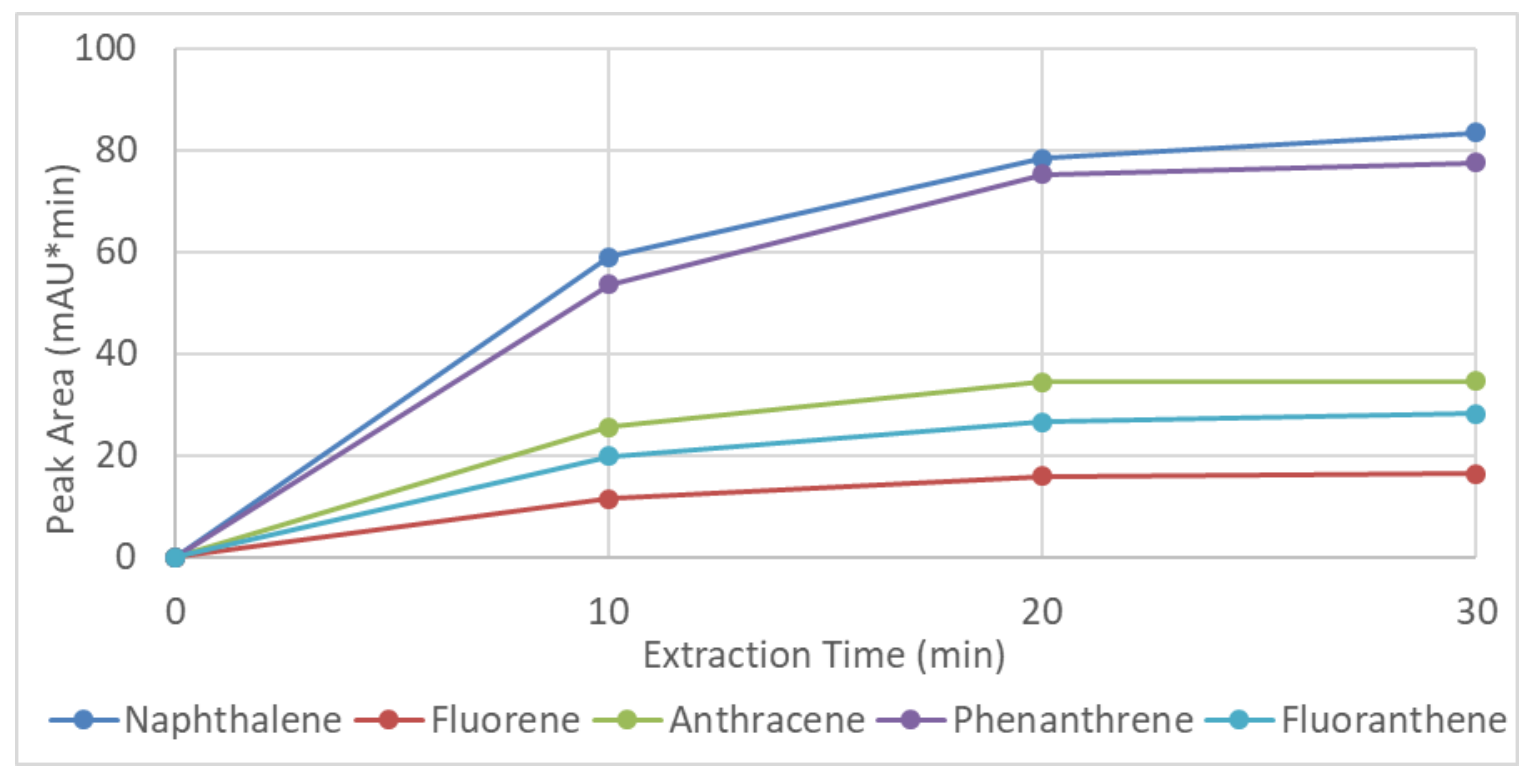

Figure 77: Experimental data of extraction time optimization of the FPSE(PDMDPS)-HPLC-UV method being developed for analysis of PAHs in aqueous samples.

The logical sequence of developing direct immersion methods is to determine the optimum back extraction time and solvent after the extraction time is optimized. Three identical sets of triplicate extractions were done from the same solution of PAHs in deionized water, only the back extraction time was different among them. Figure 78 shows the experimental data, which covers back extractions for 5,10 , and 15 minutes. It is shown in this figure that there is no benefit to be obtained from extending the back extraction beyond 5 minutes. 


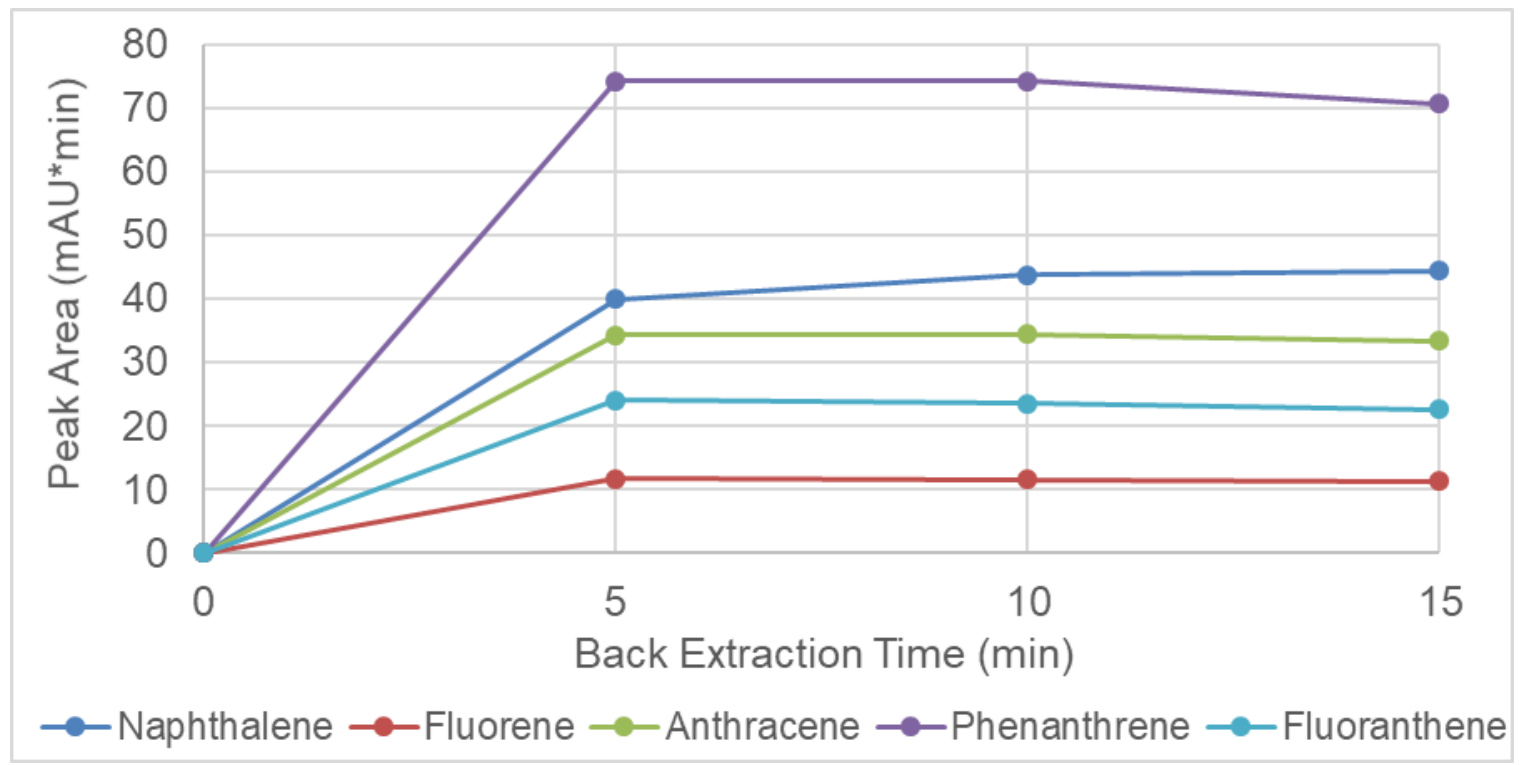

Figure 78: Experimental data of back extraction time optimization of the FPSE(PDMDPS)-HPLC-UV method being developed for analysis of PAHs in aqueous samples.

The same general procedure was followed to determine whether $\mathrm{ACN}, \mathrm{MeOH}$, or an equal part combination of each would be the best option for back extraction of the analytes. All three options were determined to be equivalent according to response magnitude of the triplicate sets of analysis. The choice for the rest of the work on PAHs was ACN.

The extraction sample volume optimization for this method yielded very interesting results, which can be seen if Fig. 79. There was very gradual increase in absolute response magnitude per $5 \mathrm{~mL}$ interval after $10 \mathrm{~mL}$ for all compounds except phenanthrene. However, the method's sensitivity level for phenanthrene is significantly higher than that of every other $\mathrm{PAH}$, and looking at the increase in response magnitude relative to the previous response, phenanthrene is not much different from the rest of the compounds. The overall (taking into account all five 
PAHs) optimum extraction sample volume in this case is $30 \mathrm{~mL}$, because there is certainly no increase in sensitivity from 30 to $35 \mathrm{~mL}$. This is the logical conclusion if sensitivity is the main focus; if it were quantitation then the optimum extraction volume would undoubtedly be $10 \mathrm{~mL}$ for concentrations high enough to be detected with the method being developed from that sample volume.

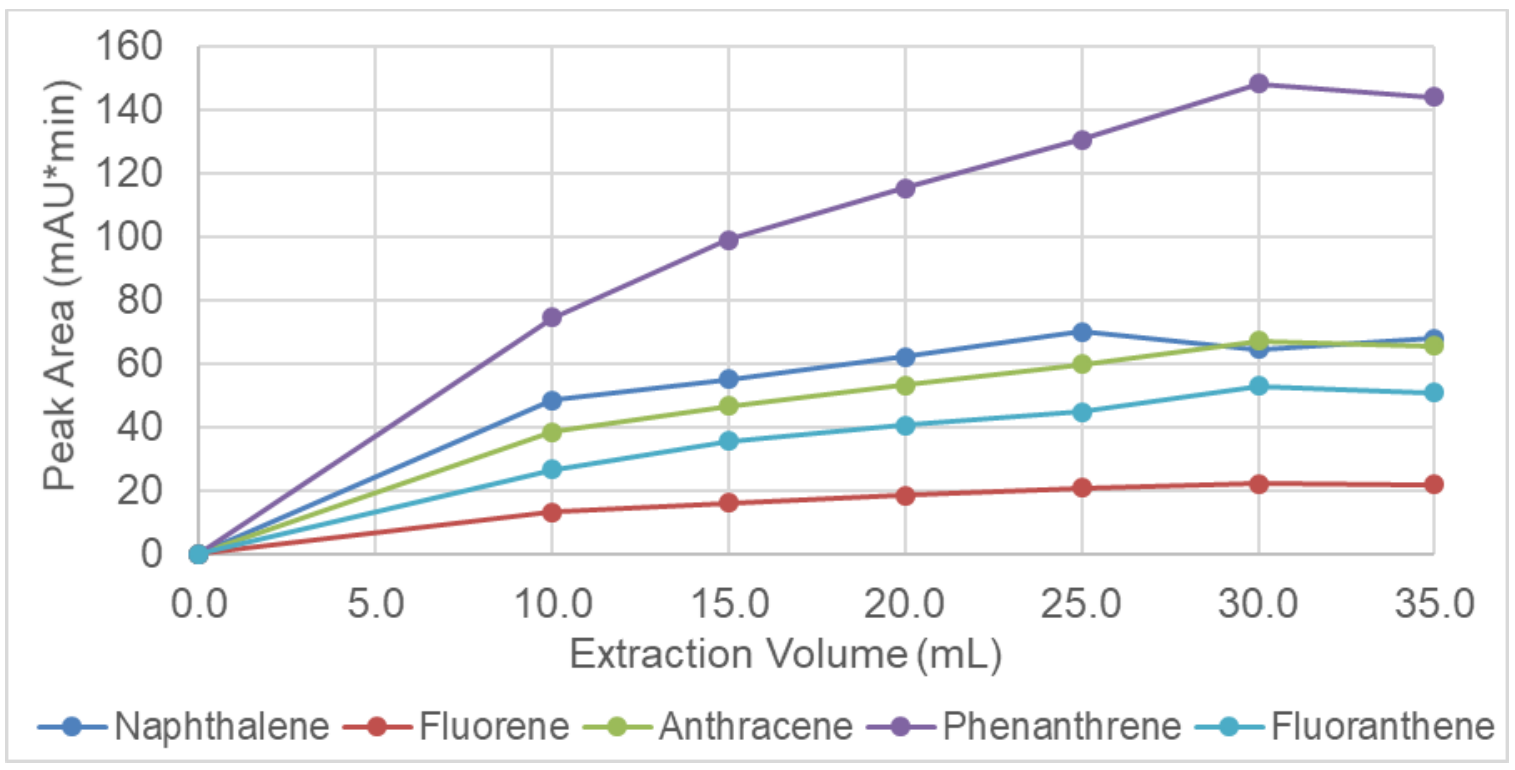

Figure 79: Experimental data of extraction sample volume optimization of the FPSE(PDMDPS)-HPLC-UV method being developed for analysis of PAHs in a aqueous samples.

\subsubsection{Figures of Merit}

A viable first step in the validation of an analytical method intended for quantitative purposes is determination of the dynamic range. The experimental results for the linearity investigation corresponding to the FPSE(PDMDPS)HPLC-UV method developed are presented in Fig. 80 for each PAH. These values are truly magnificent both in regards to the concentration range $(1-1000$ $\mathrm{ppb})$ and correlation values $\left(\mathrm{R}^{2} \geq 0.9982\right)$. This is a very important step in the 
validation of any method and it means that quantitation can be expected to be accurate within this range of concentration. There are, however, other factors to consider when performing quantitative analysis. As discussed earlier, the extraction sample volume affects the accuracy of quantitation because as it increases the partition coefficient governing the retention of analytes decreases and concentration levels can be severely underreported. Underreporting the concentration of environmental pollutants such as PAHs can be very unfortunate as it could lead to mass casualties depending on the nature of the sample being investigated. It is a criminal act.

This study was conducted by triplicate analysis of $10 \mathrm{~mL}$ aliquots of solutions of each concentration presented in Fig. 80 (1, 5, 50, 100, 250, 500, and 1000 ppb). The solutions were prepared by diluting a portion of the previous higher concentration, which would negatively affect the results. The $1000 \mathrm{ppb}$ solution was made by diluting highly concentrated stock solutions of the PAHs in ACN with deionized water. Once triplicate analyses were done of each concentration, all that was necessary was to plot the average response from each concentration vs the concentration itself. 


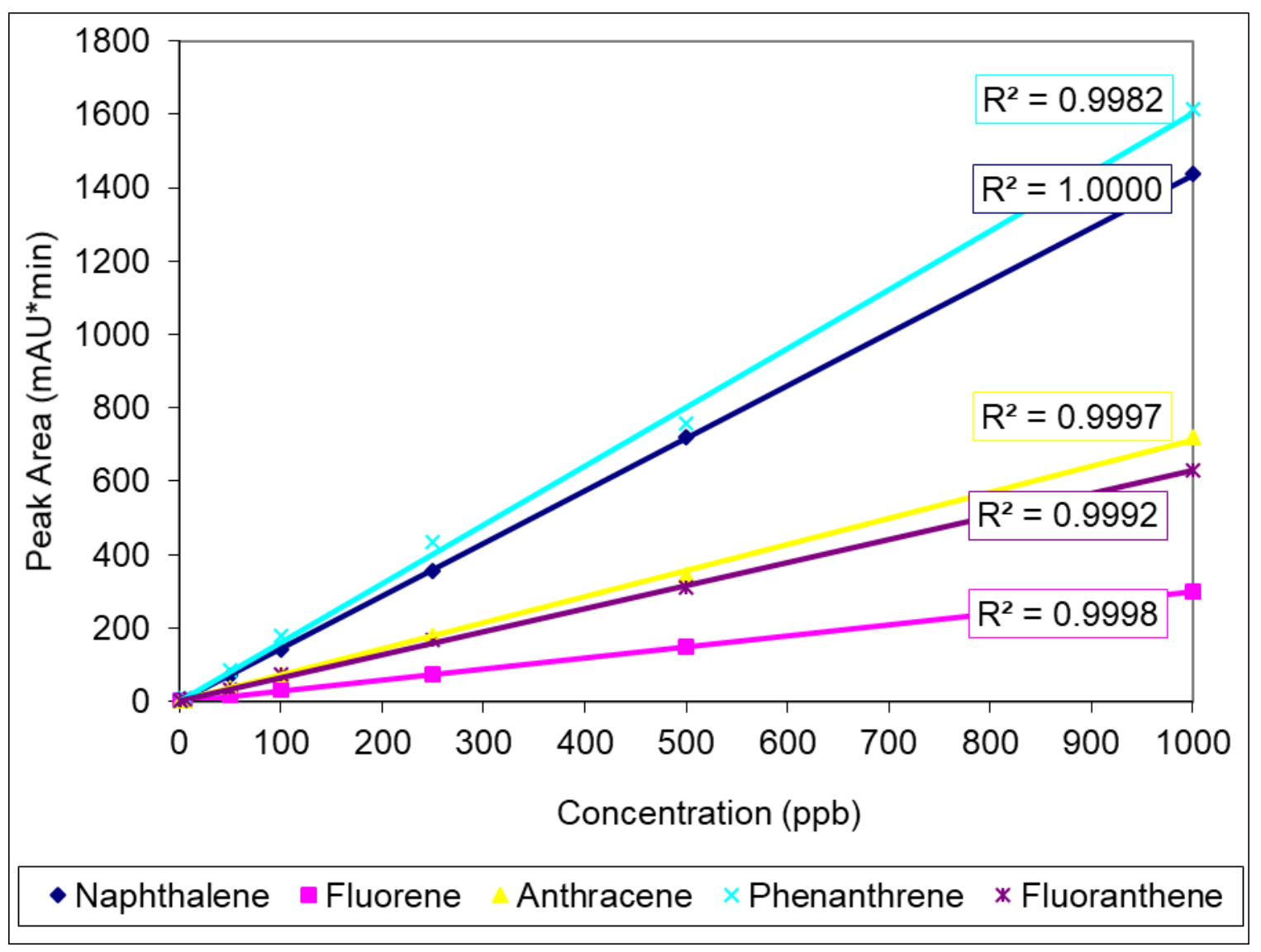

Figure 80: Experimental data of linearity study of the FPSE(PDMDPS)-HPLC-UV method developed for analysis of PAHs in aqueous samples.

The dynamic range of each compound and the corresponding $R^{2}$ value of each linearity curve are presented in Table 12 . This table also presents the results of the repeatability studies for the method being discussed, both intra and inter-day. The intraday repeatability was determined by comparing extractions of a mixture of all PAHs throughout one work day, while the inter-day variation covers three separate sets of triplicate analyses; one set per day. The repeatability of this method (2.2 - 8.4\%RSD intraday and $0.9-6.6 \% \mathrm{RSD}$ inter-day) is well within acceptable thresholds. 
Table 12: Summary of the compound specific results for intra and inter-day repeatability as well as dynamic range and the corresponding correlation coefficient for the developed FPSE(PDMDPS)-HPLC-UV method for analysis of

$\mathrm{PAHs}$ in aqueous samples.

\begin{tabular}{|c|c|c|c|c|}
\multirow{2}{*}{ Compound } & \multicolumn{2}{c}{ Repeatability (\% RSD) } & \multicolumn{2}{c}{ Linearity } \\
\cline { 2 - 5 } & Intraday & Inter-day & Range $(\mathrm{ppb})$ & $\begin{array}{c}\text { Correlation } \\
\left(\mathrm{R}^{2}\right)\end{array}$ \\
\hline Naphthalene & 2.5 & 0.6 & $1-1000$ & 1.0000 \\
\hline Fluorene & 4.6 & 0.6 & $1-1000$ & 0.9998 \\
\hline Anthracene & 2.2 & 0.9 & $1-1000$ & 0.9997 \\
\hline Phenanthrene & 3.9 & 2.9 & $1-1000$ & 0.9982 \\
\hline Fluoranthene & 8.4 & 6.6 & $1-1000$ & 0.9998 \\
\hline
\end{tabular}

The last step before applying the method to real sample matrices was to determine the LOD and LOQ for each PAH. This was done such that the signalto-noise ratio, $\mathrm{S} / \mathrm{N}$, is equal to 3 for the LOD and 10 for the LOQ. The compound specific values for both LOD and LOQ are presented in Table 13 for this method as well as another found in a 2016 article in Talanta by Zhang et al [142]. The sample preparation of this method, which used GC-MS for instrumental analysis, was a novel device that they called ST-SPME (sintered titanium SPME) with a PDMS coating [142]. This device was made by the Zhang et al. research group in an effort to improve the porosity and by extension speed up the mass transfer and increase the PCSA and sorbent loading capacity [142]. It is interesting that they chose to call this a new version of SPME because the substrate is a disk, not a rod (Fig. 81) [142]. ST-SPME further deviates from traditional SPME, 
perhaps more than any other variation of the traditional device, by pushing the sample matrix through the substrate [142] instead of simply exposing the sorbent to the headspace or by direct immersion. ST-SPME effectively attempts absolute extraction instead of equilibrium extraction, which is unlike any other SPME related technique and a fundamental shift from it. The inventors of ST-SPME could easily have called it ST-SPE if the device were larger because the device and the type of extraction certainly resembles SPE more than SPME.

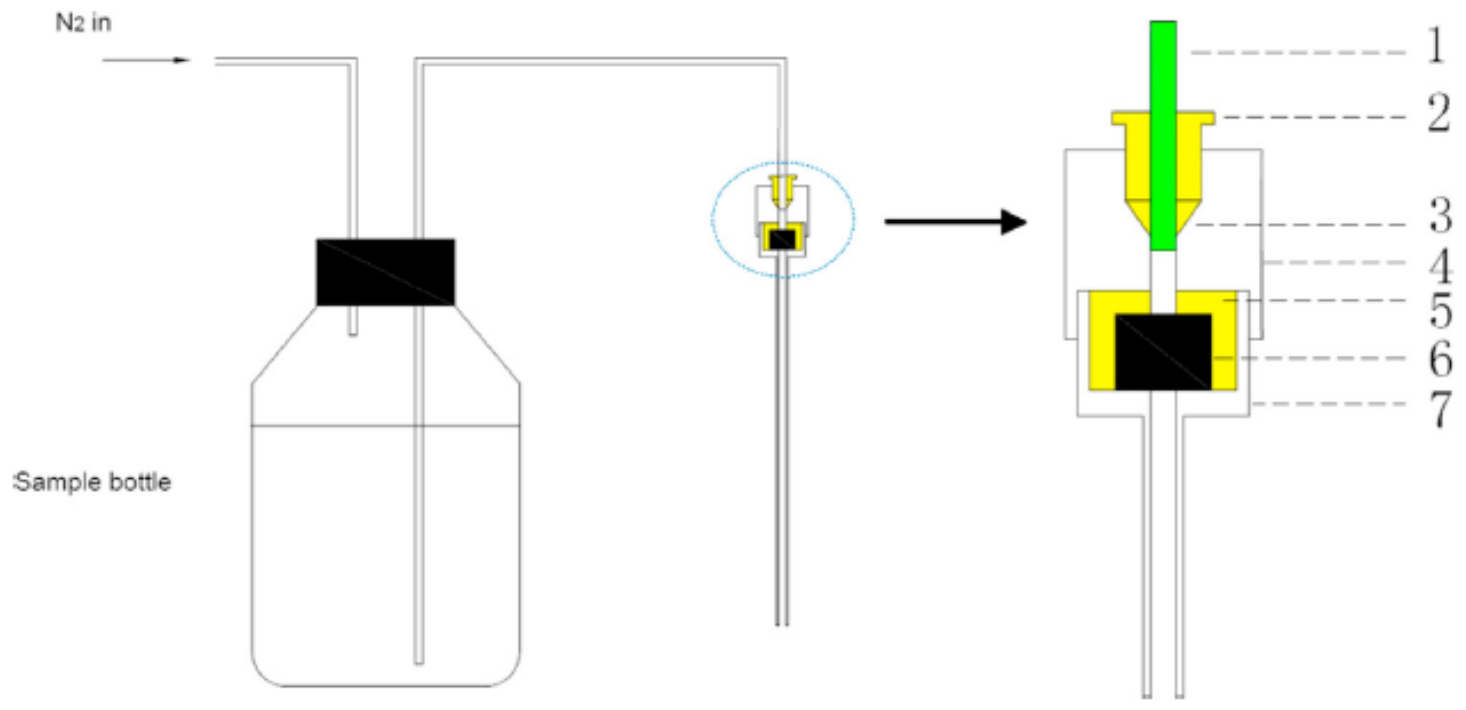

Figure 81: Apparatus used to coat and evaluate the ST-SPME disk: (1) PEEK tube, (2) PEEK screw, (3) PEEK ferrule, (4) stainless steel nut, (5) PEEK ferrule, (6) sintered titanium disk, and (7) stainless steel screw.

As shown in Table 13, despite the innovation of this new version of SPME, the LOD and LOQ values obtained with the developed FPSE(PDMDPS)-HPLC-UV method are better for each compound except fluoranthene. For this compound the values of each method are comparable to each other. It is remarkable that the FPSE based method presented here outperforms this ST-SPME-GC-MS 
method as is because the compounds are thermally desorbed off the ST-SPME disk and all are introduced to the GC column. In the FPSE method only a small portion (1\%) of the back extracted solution is injected in the HPLC-UV.

Table 13: Compound specific results for experimental calculations of LOD and LOQ for the developed FPSE(PEG)-HPLC-UV method and cmparison to performance of a method in the literature.

\begin{tabular}{|c|c|c|c|c|}
\hline Compound & LOD (ppt) & LOQ (ppt) & $\begin{array}{c}\text { LOD (ppt) } \\
\text { Zhang et al. }\end{array}$ & $\begin{array}{c}\text { LOQ (ppt) } \\
\text { Zhang et al. }\end{array}$ \\
\hline Naphthalene & 0.0073 & 0.0244 & 0.2 & 0.6 \\
\hline Fluorene & 0.167 & 0.556 & 0.3 & 1.1 \\
\hline Anthracene & 0.0634 & 0.211 & 0.1 & 0.4 \\
\hline Phenanthrene & 0.0203 & 0.0678 & 0.1 & 0.2 \\
\hline Fluoranthene & 0.0995 & 0.332 & 0.1 & 0.3 \\
\hline
\end{tabular}

Lots of other methods have been developed for analysis of PAHs from aqueous solutions, and newly developed methods are invariably published with LOD values because of the importance of sensitivity. The detection limits of several methods published in peer-reviewed journals are summarized in Table 14, as well as the extraction sample volume and the methodology used for both sample preparation and instrumental analysis. 
Table 14: Comparison of the LOD range for the developed FPSE(PEG)-HPLC-

UV method to that of eight methods in the literature. The ranges reported for each method correspond to several PAHs, not necessarily the same included in the research presented herein.

\begin{tabular}{|c|c|c|c|}
\hline Methodology & $\begin{array}{c}\text { Sample } \\
\text { Volume (mL) }\end{array}$ & $\begin{array}{c}\text { Extraction } \\
\text { Time (min) }\end{array}$ & $\begin{array}{c}\text { LOD Range } \\
\text { (ppt) }\end{array}$ \\
\hline SBSE'-HPLC-FLD [143] & 10 & 140 & $0.2-1.5$ \\
\hline SPME(PDMS)-GC-MS [144] & 30 & 45 & $1-29$ \\
\hline MA-HS-SPMEb-GC-FID [145] & 20 & 30 & $30-1000$ \\
\hline SPMEc-GC-MS/MS [146] & 18 & 60 & $0.07-0.76$ \\
\hline SPME-GC-FID [147] & 15 & 30 & $20-40$ \\
\hline ACIT-SPMEd-GC-FID [148] & 350 & 2 & $0.13-0.86$ \\
\hline SBSE-HPLC-FLD [149] & 500 & 60 & $0.05-3.41$ \\
\hline ST-SPME'-GC-MS [142] & 100 & 2 & $0.06-3.2$ \\
\hline FPSEg-HPLC-UV & 10 & 20 & $0.0073-$ \\
\hline
\end{tabular}

a10 $\mathrm{mm}$ long, $1 \mathrm{~mm}$ thick PDMS coating

bPDMS/DVB sorbent. Microwave Assisted Headspace SPME.

cPDMS/DVB sorbent

${ }^{d}$ Bundle of glass capillaries coated with PDMS sorbent inside a quartz liner.

e20 mm long, $1 \mathrm{~mm}$ thick sol-gel PDMS coating.

fSintered Titanium disk SPME. PDMS sorbent.

gOriginal research being presented herein. 
Most of the methods mentioned in Table 14 use some version of gas chromatography and thermally desorb all the analytes into the separation column, which presents them with a functional advantage over the developed FPSE(PDMDPS)-HPLC-UV method which only introduces $1 \%$ of the back extraction solution into the HPLC-UV. FPSE media exhibit such greatly desirable features for sample preparation, however, that the method being presented here is still more sensitive than all the others in Table 14. PAHs are toxic at very low concentrations in environmental waters, so the importance of this cannot be overstated, especially considering that this FPSE method also displays great repeatability and ease of use.

\subsubsection{Ultra-Sensitive Alternate FPSE Procedure}

The procedure of carrying out FPSE based analysis is very simple, not very laborious, and many extractions can be performed simultaneously. An alternate procedure was investigated, however, that is a bit more time consuming and laborious but increases the sensitivity of the analysis significantly. In this alternate approach, nothing changes regarding the extraction. The back extractions, however, are different because three FPSE media are introduced in the same aliquot of $500 \mu \mathrm{L}$ of eluting solvent. This is done sequentially. Three identical extractions are performed, from three separate aliquots of the same solution if possible, then one is submerged in the solvent used for back extraction. When the predetermined time allotted for back extraction has passed, the FPSE media is removed and another is placed in the same solution that was just back extracted. This is done again for the third FPSE media. This approach 
increases the concentration in the final back extracted solution because it introduces the analytes retained by all three FPSE media in one aliquot of solvent that would normally be used for just one FPSE media. The chromatogram shown previously in Fig. 76 in subsection 5.6 .3 is a result of this alternate FPSE procedure. It is evident that the sensitivity is indeed increased because the peaks are all relatively large, clearly much larger than the noise, and the concentration from the solution analyzed was 200 ppt.

\subsubsection{Field Application to Environmental Samples}

The developed and validated method for analysis of PAHs from water was applied to the direct extraction of these compounds from three relevant real aqueous matrices: pond water, reclaimed water, and saline water from a local beach. It is important to note that these extractions were done without any pretreatment whatsoever of the matrix. The pond and saline water samples in particular had lots of particles in them large enough to be visible. FPSE media can extract from these samples without any modification like filtering because of the large available PCSA.

The first task of this study was to perform a method blank on each environmental water sample. None of the PAHs were detected in any of these samples so they were spiked to a concentration of $5 \mathrm{ppb}$ to determine the effectiveness of the method with these relatively complex sample matrices. Each spiked water sample was analyzed in triplicate, the sample volume being $10 \mathrm{~mL}$. Once the instrumental analysis portion of the method was completed for each sample 
matrix the peaks in the resulting chromatograms were integrated and the relative recoveries were calculated for each compound. These results are all presented in Fig. 82.

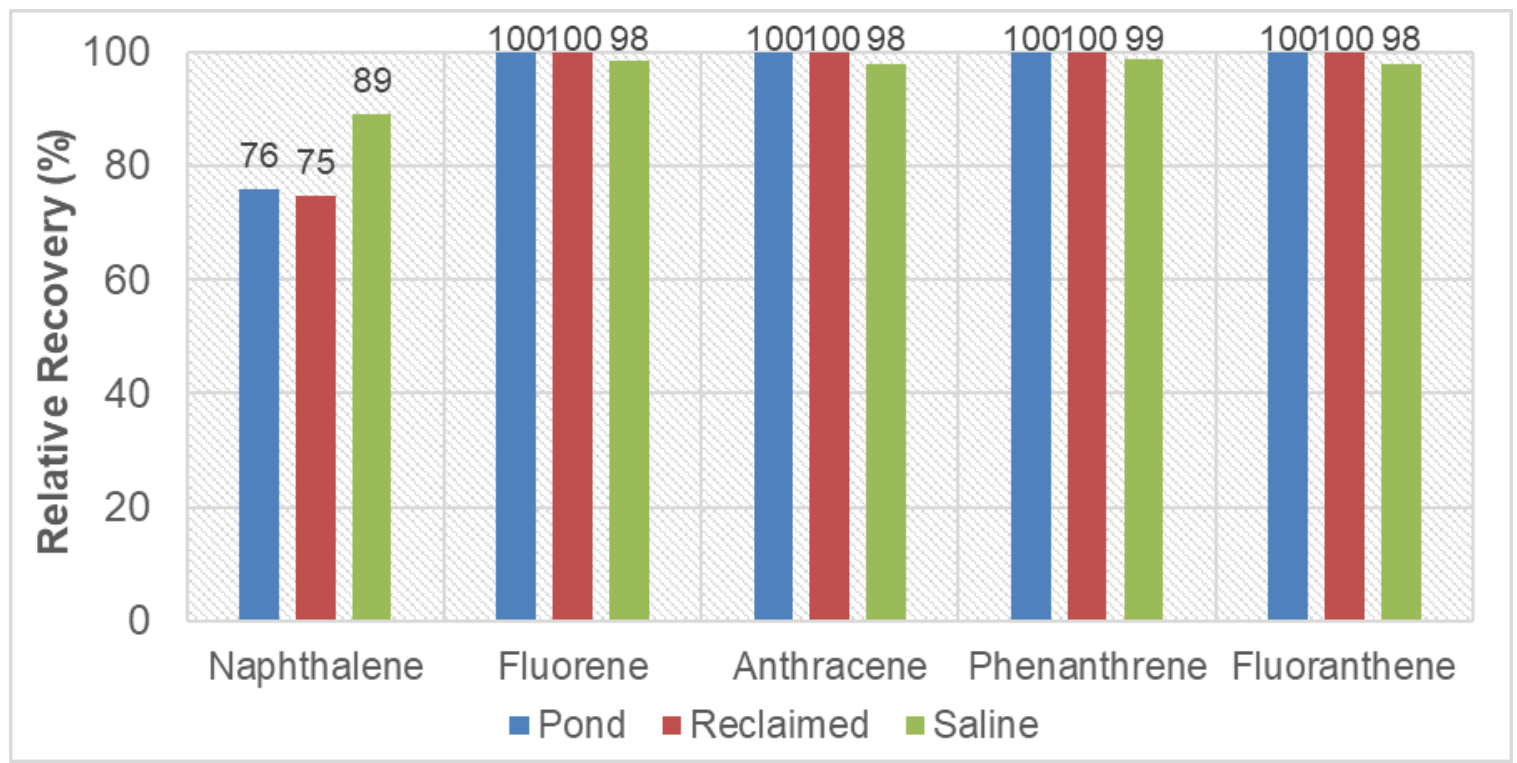

Figure 82: Compound specific relative recovery percentages of each of the five

PAHs from pond, reclaimed, and saline water samples, respectively.

The results of the application of the method to real samples are yet another indication that this method is a great option for analysis of PAHs in aqueous samples worthy of being considered a leading possibility to improve upon current regulatory methods. As shown in Fig. 82, the relative recoveries of the developed FPSE based method are just about perfect for fluorene, anthracene, phenanthrene, and fluoranthene. Naphthalene is the only compound where there is a noticeable negative bias when analyzing pond, reclaimed, and saline water samples, meaning it likely reacted with some other chemical species in those samples or formed a complex with particles. Naphthalene is the only one of the five PAHs that only has two carbon rings and is also a significantly smaller 
molecule, which is likely why it reacted with something else in those samples when the others didn't.

\subsection{Endocrine Disrupting Chemicals in Milk}

Developing an FPSE based method for analysis of EDCs in milk is more challenging than the previously described methods and more extensive work was done with it both in terms of development and validation. Tap water, reclaimed water and fresh and saline environmental water samples all present very real challenges but milk is at a different level altogether. Milk is much denser and contains very large molecules such as fats and proteins in addition to sugars, minerals, other chemical species that may interfere with extraction of EDCs.

As a consequence of these analytical challenges and the immense importance of milk as a vital nutrition source worldwide, the following method, developed for the six EDCs in Table 5 but expected to be applicable for any natural or synthetic hormone not included here, is perhaps even more important than the ones presented previously.

\subsubsection{Preparation of Standard Solutions}

Each of the six EDCs included in this report were purchased in bulk, solid form. Standards were prepared by dissolving each analyte in acetonitrile to a concentration of $10,000 \mathrm{ppm}$, except for estrone, for which the concentration was $2,000 \mathrm{ppm}$ because its solubility is lower than that of the rest. Once these highly concentrated standard solutions were prepared, an intermediate solution of all 6 analytes together was made, the concentration being $50 \mathrm{ppm}$ of each EDC. This 
solution was used for preparation of more diluted standards while developing the instrumental methods. For the purpose of spiking milk or other matrices for method development or validation, a different intermediate was made. The concentration of this one was $400 \mathrm{ppm}$ for BPA, HEX, and DES; the individual concentration of E2, EE2, and E1 was $200 \mathrm{ppm}$. Another intermediate of concentration $500 \mathrm{ppm}$ of each of the EDCs mixed was prepared for the sorbent capacity study. Dilutions of standard solutions for instrumental method development or spiking of samples were always kept to a minimum to diminish error. Standards and intermediates were always kept refrigerated.

\subsubsection{Instrumental Method Development}

The first step in the method development process was development of the instrumental part of the method. This was done by injecting standard solutions of the EDCs individually to confirm the ability of the instrument to detect each analyte and the elution order through the selected Zorbax Extend-C18 column. Once the elution order was determined, satisfactory resolution of the peaks was achieved with an isocratic method (55\% water, $45 \%$ acetonitrile; flow rate 1 $\mathrm{mL} / \mathrm{min}$; column compartment temperature $30^{\circ} \mathrm{C}$; VWD set at $200 \mathrm{~nm}$ ). Fig. 83 shows a representative chromatogram of an extraction of the 6 EDCs from whole milk where the concentration of BPA, E2, and E1 in the milk was $200 \mathrm{ppb}$ and the concentration of EE2, HEX, and DES was $100 \mathrm{ppb}$. The injection volume was 15 $\mu \mathrm{L}$. 


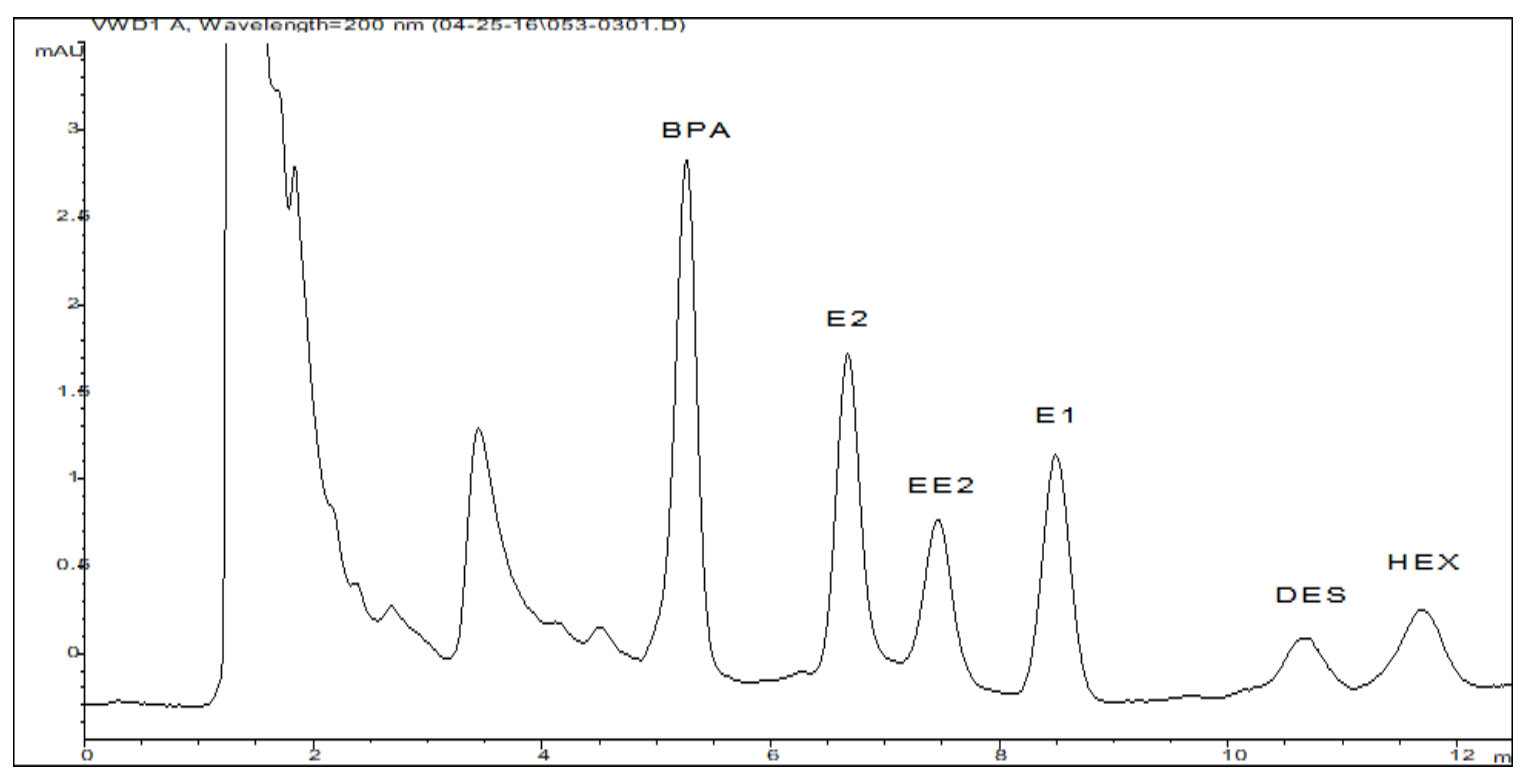

Figure 83: Representative HPLC-UV chromatogram of an FPSE extraction of the 6 EDCs from whole milk. The concentration of BPA, E2, and E1 in the milk was $100 \mathrm{ppb}$, the concentration of EE2, HEX, and DES was $200 \mathrm{ppb}$, and the injection volume was $15 \mu \mathrm{L}$.

\subsubsection{Determination of Optimum FPSE Media Sorbent}

The endocrine disruptor chemicals included in the research presented here are not highly polar, instead range from being slightly polar to nonpolar, as seen in Table 6 via the octanol-water partition coefficient of each. In response to this range of polarity, two FPSE media were selected as possible choices for extraction of these compounds from whole milk: FPSE-PTHF (medium polarity) and FPSE-PDMS (nonpolar). The experimental data resulting from this comparison is shown in Fig. 84, where it is obvious that while both are effective to some extent, the better choice of the two is FPSE-PTHF. 


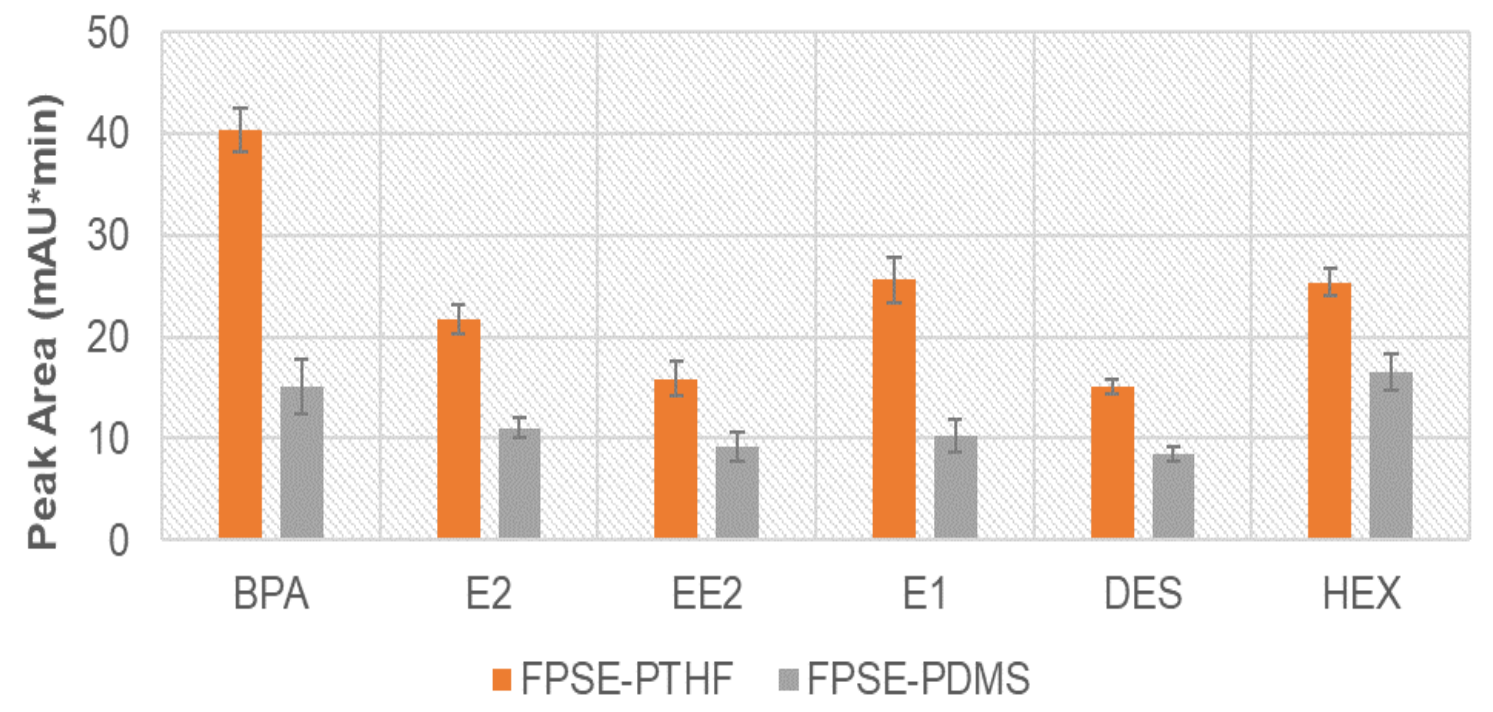

Figure 84: Experimental data of the comparison of the effectiveness of FPSEPTHF and FPSE-PDMS media, respectively, for extraction of EDCs from whole milk.

\subsubsection{Optimization of Sample Preparation Parameters}

After knowing which FPSE media would be used for the method, the specific parameters had to be optimized. In order to determine each optimum parameter, fresh, locally bought whole milk was always spiked with the six pertinent EDCs. Comparisons were made in triplicate where the only parameter being changed was the one being optimized. The optimum parameters were chosen based on sensitivity, repeatability and efficiency.

The extraction time was optimized first. How long does it take for the sol-gel PTHF sorbent of the selected FPSE media to retain as much of the endocrine disruptors as possible? Triplicate extractions were done, all within one hour of each other, with various extraction times: 10, 20, 30, 40, 50 and 60 minutes (Fig. 85). With each increment in time the results were more sensitive until 60 minutes 
when the response for each compound increased less than $10 \%$ when compared to the magnitude of the response at 50 minutes. Therefore, the optimum extraction time for maximum sensitivity is $\mathbf{5 0}$ minutes. It should be noted that when using this method, a different extraction time may serve certain needs just as well while making the process shorter.

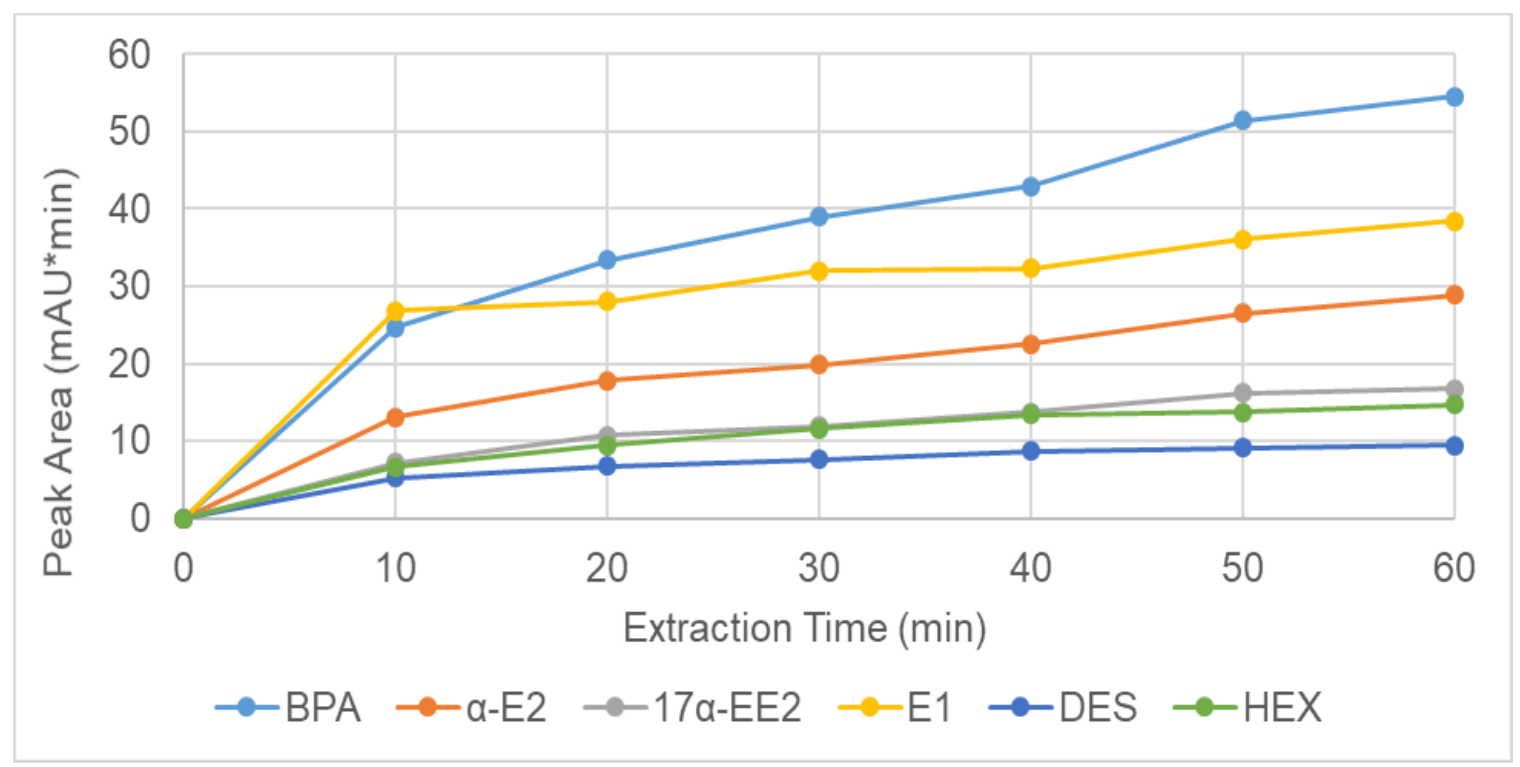

Figure 85: Experimental data of extraction time optimization of the FPSE(PTHF)HPLC-UV method being developed for analysis of 6 EDCs from whole milk. Similarly, the optimum back extraction time was determined to be 5 minutes (Fig. 86), and acetonitrile was chosen as the back extraction solvent over methanol and a 1:1 mixture of each. Methanol interfered with the first peak of the HPLC-UV chromatogram by creating a larger void volume disturbance since it is UV active at the wavelength of the method $(200 \mathrm{~nm})$. 


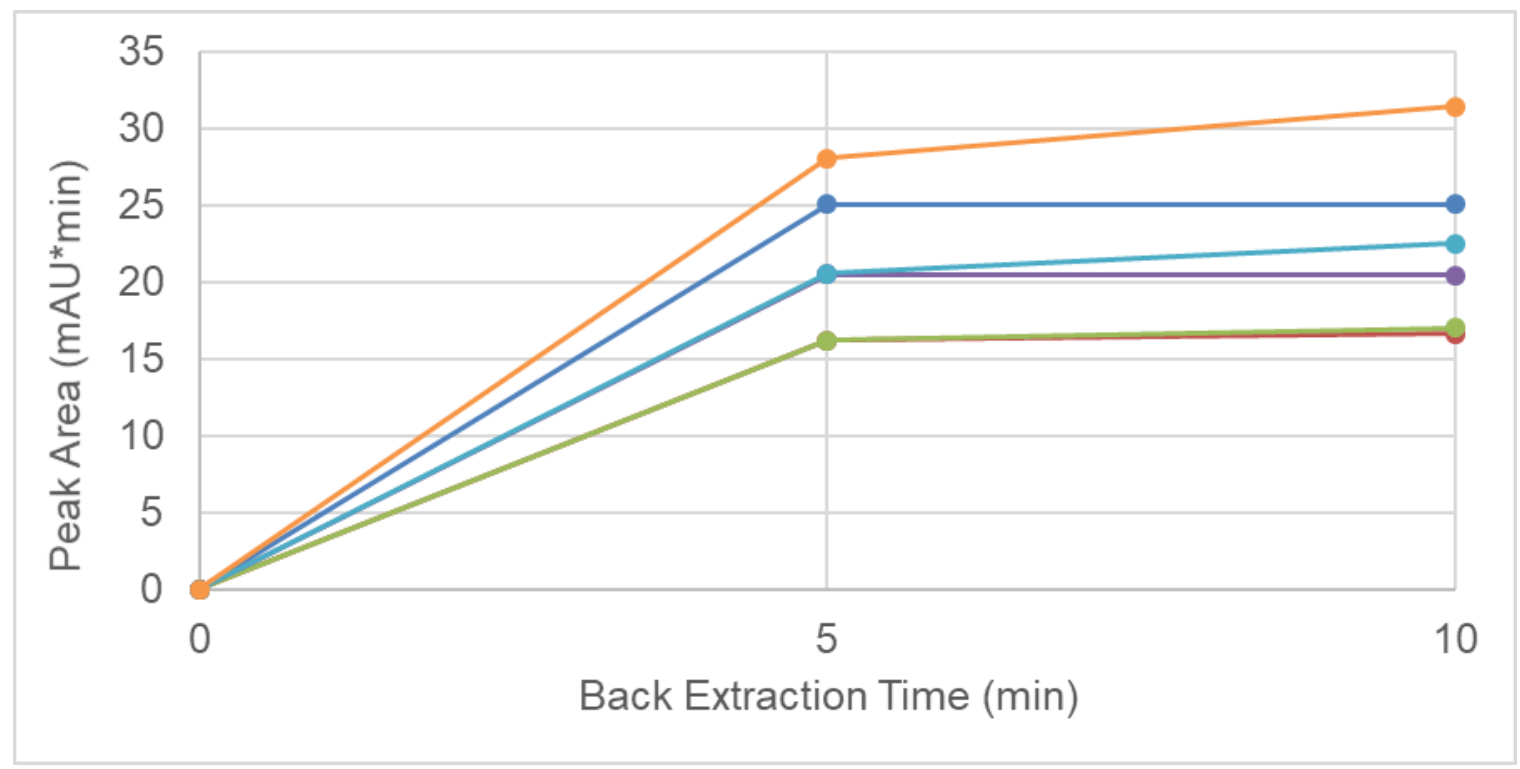

Figure 86: Experimental data of back extraction time optimization of the FPSE(PTHF)-HPLC-UV method being developed for analysis of 6 EDCs from whole milk.

The optimum volume of extraction solution was determined to be $5 \mathrm{~mL}$ (Fig. 87).

This result for optimum extraction volume for this method is quite convenient since quantitation is best at lower volumes. This is the only one of the five methods presented here where the optimum extraction volume is the among the quantities tested. 


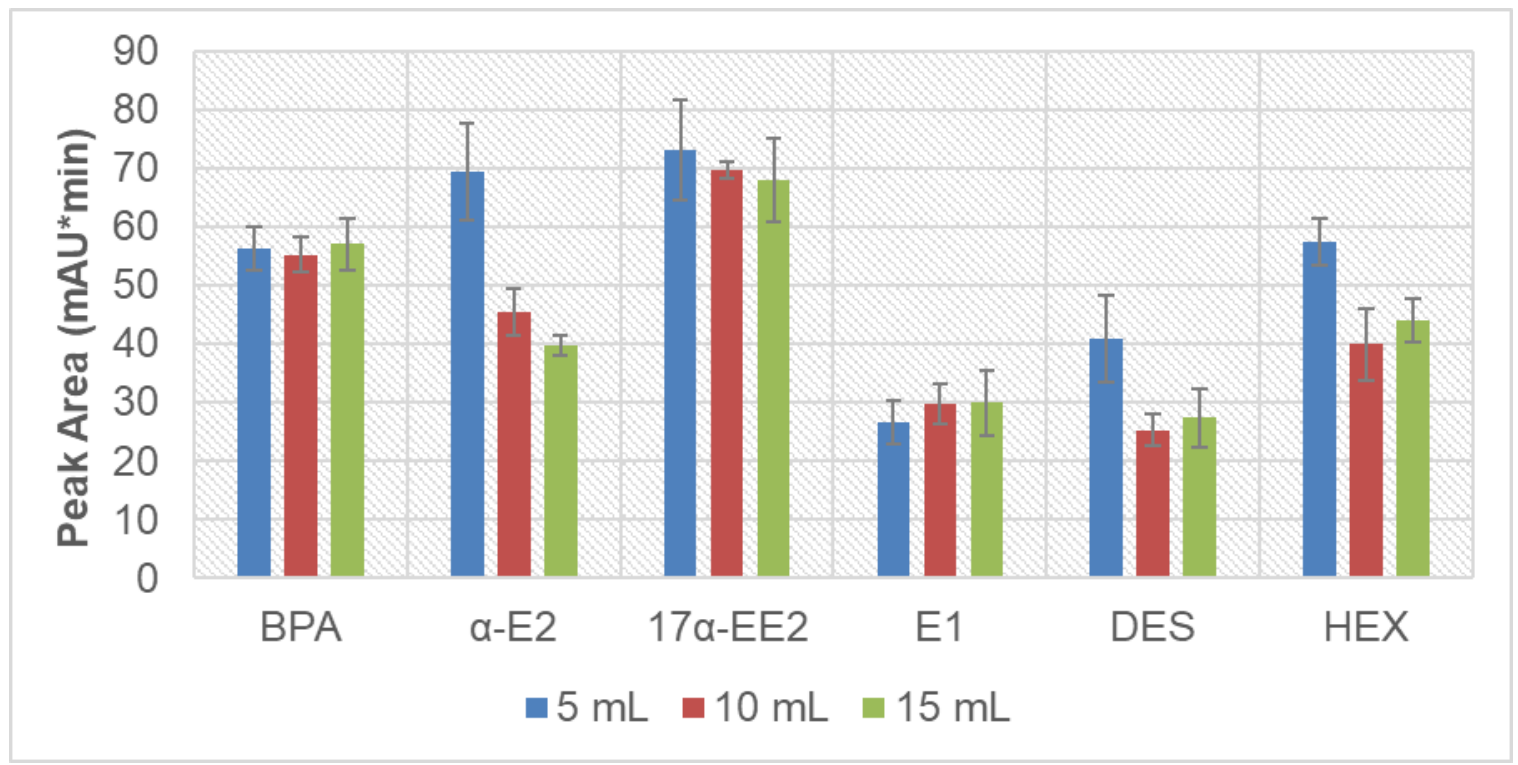

Figure 87: Experimental data of extraction volume optimization of the FPSE(PTHF)-HPLC-UV method being developed for analysis of 6 EDCs from whole milk.

The injection volume was also optimized such that the response is as high as possible without significantly sacrificing the quality of any of the chromatographic parameters that lead to acceptable resolution: capacity, selectivity and efficiency. The best option was $15 \mu \mathrm{L}$. Lower injection volumes provided smaller responses and higher ones caused significant overlapping of the void volume disturbance and the peak for BPA. Notwithstanding the complexity and challenging nature of extracting from whole milk without pretreating it, superb selectivity was achieved, as evidenced by the chromatographic quality presented. The peaks for the 6 EDCs were clearly separated with high resolution, exhibit good peak symmetry and there are no background interferences of consequence despite injecting a relatively high volume. 
Extractions of small organic molecules directly from whole milk had not been achieved at the time the method being presented here was developed. This lack of precedent demanded a more rigorous method development process than much simpler aqueous samples. At this point in the process many parameters had been set, but even more were investigated. Stirring speed, which is always used in FPSE extractions to apply external stimuli, is one of those parameters. A digital stirring plate was used, and sets of triplicate analysis were done with the FPSE(PTHF)-HPLC-UV method to the extent that it had been developed at this time. The only difference between the triplicate sets of analysis was the stirring speed. Fig. 88 presents the experimental results of this study.

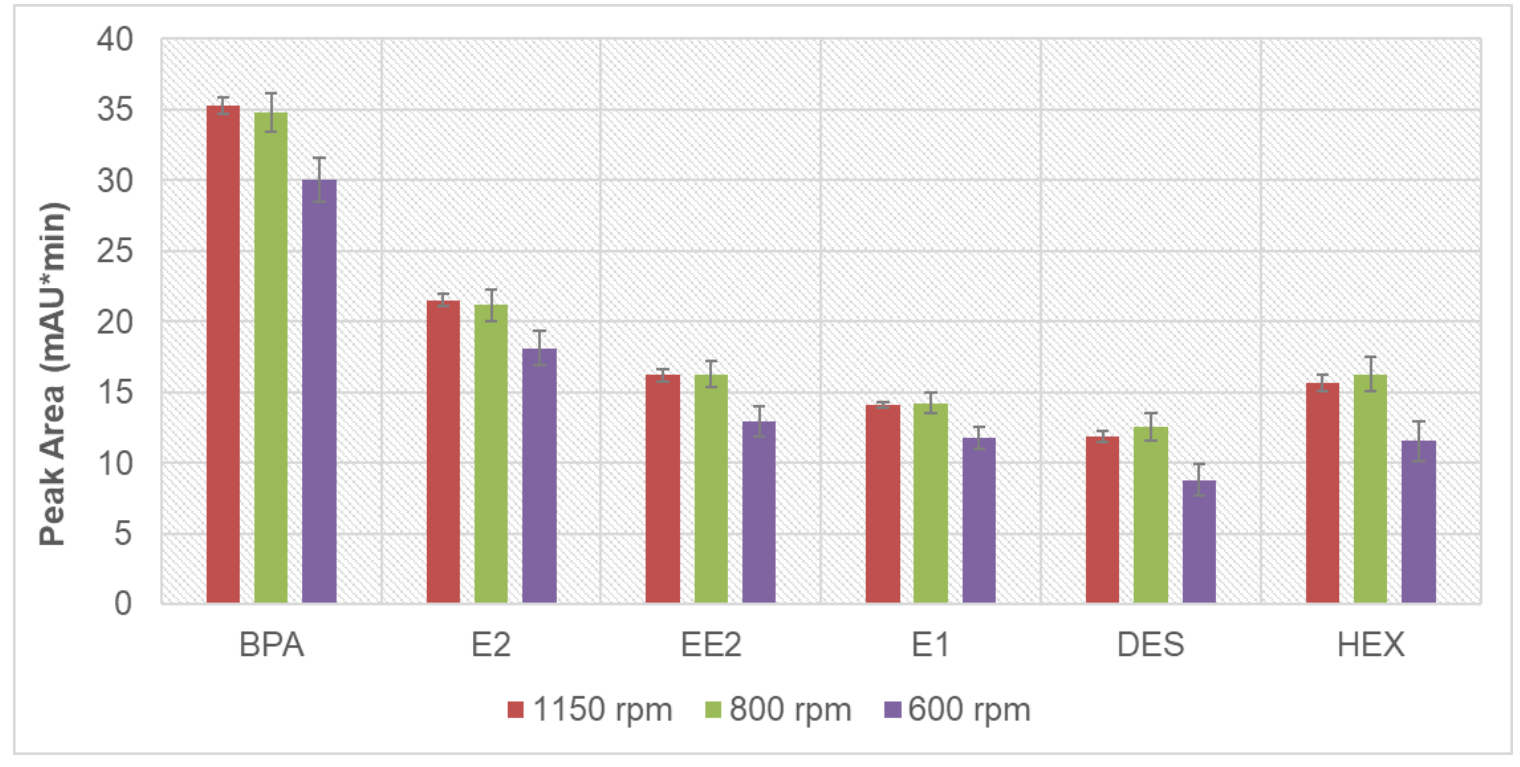

Figure 88: Experimental results of stirring speed optimization of the FPSE(PTHF)-HPLC-UV method being developed for analysis of 6 EDCs from whole milk.

Interestingly, there is not much difference between stirring at 600,800 , and 1150 rpm (the maximum speed of the instrument). There is definitely not a significant 
difference between the effectiveness of the method at 800 and $1150 \mathrm{rpm}$ and it is possible that even the effectiveness at $600 \mathrm{rpm}$ is equivalent statistically. Ultimately the chosen stirring speed was $800 \mathrm{rpm}$ because the optimum volume $(5 \mathrm{~mL})$ is relatively low and the stirring at $1150 \mathrm{rpm}$ can push the FPSE media up the wall of the extraction glass vials and partially out of the sample. This concludes the method development phase of this research as it pertains to analysis of EDCs in whole milk.

\subsubsection{Figures of Merit}

After rigorous development, the method was ready for validation. The repeatability was tested intra $(n=5)$ and inter-day $(n=3)$ and a dynamic range covering three orders of magnitude was determined with a seven point linearity curve for each of the six EDCs. All of these studies were done in triplicate.

The repeatability studies were performed following the design described previously, except that it was all done with milk as the sample matrix instead of using standard solutions in deionized water. For intraday repeatability five sets of analysis were performed during the same day. For each set a fresh sample of whole milk was spiked to a concentration of $200 \mathrm{ppb}$ of BPA, E2, and E1 and 400 ppb of EE2, DES, and HEX. The same principle of preparing fresh milk solutions at that concentration was used for inter-day repeatability, which meant comparing analysis results of triplicate sets performed on three different days. Table 15 shows the results of both repeatability studies. The repeatability is rather impressive. In fact, the \% RSD values calculated based on the experimental data 
would be acceptable even if deionized water was the sample matrix. Of course, milk is the sample matrix in this case and it is not only much more challenging to analyze than deionized water but one of the most difficult and complex matrices of any kind.

Table 14 also includes the experimental results of the linearity study $(n=7)$. Both the confirmed dynamic range and corresponding correlation coefficients are displayed for each EDC. The dynamic range for BPA, E2, and E1 is $25-10000$ ppb, half as much as the dynamic range for EE2, DES, and HEX (50 - 20000 $\mathrm{ppb})$. This is not at all surprising because early in method development it was determined that the method as a whole (including the instrument's performance) is much more sensitive for analysis of BPA, E2, and E1 than EE2, DES, and HEX. The correlation coefficients $(0.9983-0.9992)$ are as good as can be expected for analysis of any kind of solution. Considering that these are results of an analytical method for analysis of whole milk, these values are truly remarkable. 
Table 15: Summary of the compound specific results for intra and inter-day repeatability as well as dynamic range and the corresponding correlation coefficient for the developed FPSE(PTHF)-HPLC-UV method for analysis of EDCs in whole milk.

\begin{tabular}{c|c|c|c|c|}
\multirow{2}{*}{ Compound } & \multicolumn{2}{c}{ Repeatability (\% RSD) } & \multicolumn{2}{c}{ Linearity } \\
\cline { 2 - 5 } & Intraday & Inter-day & Range $(\mathrm{ppb})$ & $\begin{array}{c}\text { Correlation } \\
\left(\mathrm{R}^{2}\right)\end{array}$ \\
\hline BPA & 3.6 & 12.8 & $25-10000$ & 0.9983 \\
\hline E2 & 8.1 & 4.5 & $25-10000$ & 0.9989 \\
\hline EE2 & 8.9 & 9.3 & $50-20000$ & 0.9992 \\
\hline E1 & 3.9 & 7.2 & $25-10000$ & 0.9992 \\
\hline DES & 13.9 & 8.8 & $50-20000$ & 0.9985 \\
\hline HEX & 8.6 & 11.3 & $50-20000$ & 0.9986 \\
\hline
\end{tabular}

In an effort to further validate the developed FPSE-HPLC-UV method for analysis of EDCs from raw milk, the sorbent loading capacity was tested. FPSE is a viable sample preparation option not only for extraction of analytes from complex, untreated samples such as milk but also for retention of a large quantity of analytes due to the high PCSA provided by the inherently porous sol-gel polyTHF sorbent. The results shown in Fig. 89 provide irrefutable evidence that this hypothesis is correct. This is a very significant finding because while it was known that FPSE media's sorbent loading capacity is extraordinary $\left(3.96 \mathrm{mg} / \mathrm{cm}^{2}\right.$ or $19.8 \mathrm{mg}$ per $5 \mathrm{~cm}^{2}$ unit), this result shows that even if some of the active sites on the sorbent are blocked by components of milk such as proteins or fat, plenty of the sorbent is remains available. 


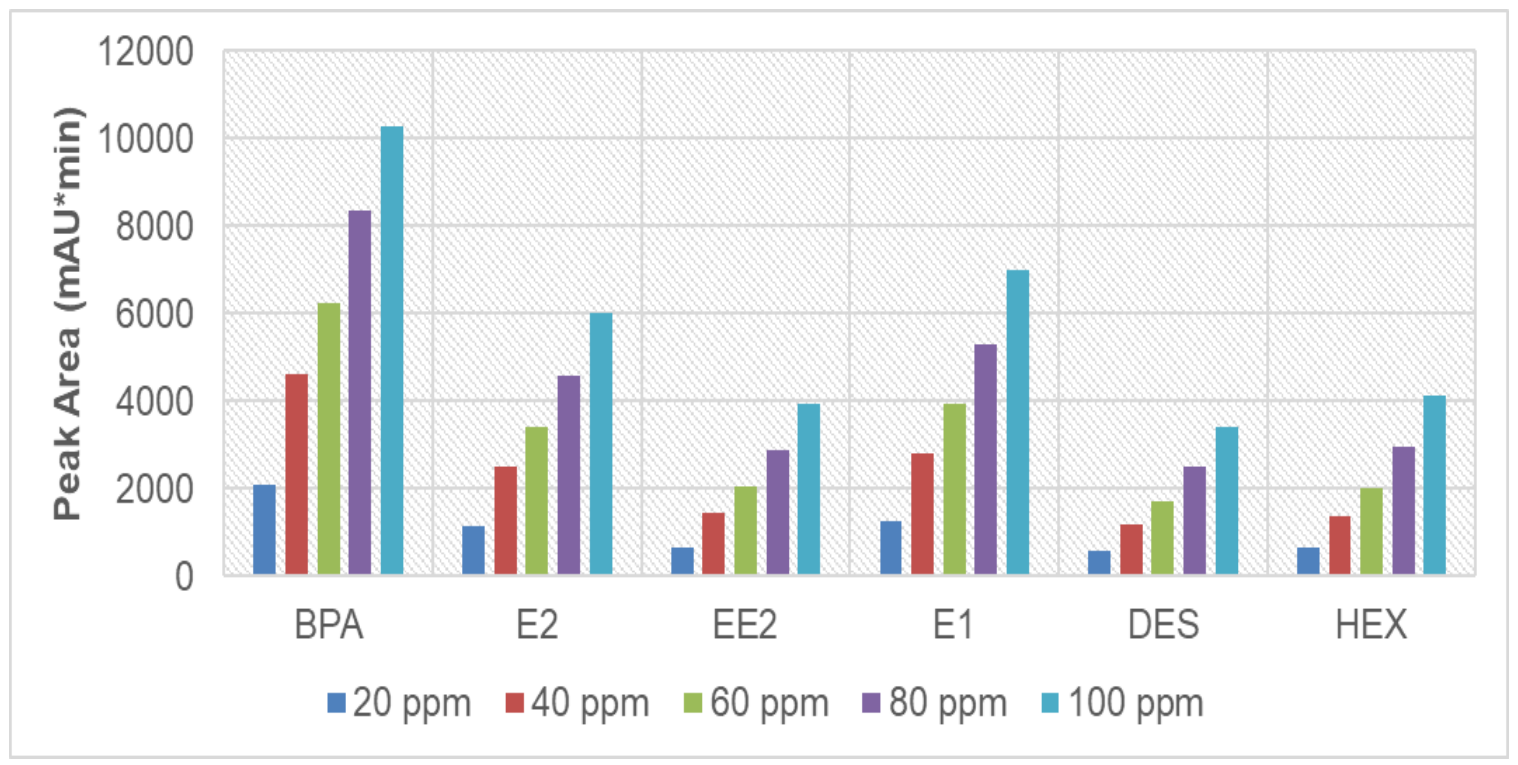

Figure 89: Results of sorbent loading capacity study of the developed FPSE(PTHF)-HPLC-UV method for analysis of 6 EDCs from whole milk.

The LOD and LOQ were determined such that the signal-to-noise ratio $(\mathrm{S} / \mathrm{N})$ is equal to 3 for LOD and 10 for LOQ with the HPLC-UV that was used for method development. A recent article by Gañán et al. in the J. of Chromatography A [150] presents mesoporous silica SPE cartridges as an improvement upon classic sorbents such as amorphous silica and polymeric materials for analysis from complex matrices such as milk. However, as shown in Table 15, the MQL values reported in this article (100 - $630 \mathrm{ppb})$, which used SPE with mesoporous silica coupled to HPLC-DAD, are much higher than the LOQ values being reported here with the developed FPSE(PTHF)-HPLC-UV method (3.45-21.6 $\mathrm{ppb})$. This values of this SPE-HPLC-DAD method are even after evaporation of the elution solvent ( $2 \mathrm{~mL}$ of methanol) and reconstitution to $150 \mu \mathrm{L}$ (theoretical preconcentration factor of 6.7). 
Table 16 also includes the calculated LOD and LOQ values of a Hollow Fiber Liquid Phase Microextraction (HF-LPME) method coupled to HPLC-DAD/FD, in which for some analytes the diode-array detector was used and for others the fluorescence detector [151]. This method achieved much better quantitation values $(2.7-269)$ than the one utilizing Acetate-MeOH-SPE-HPLC-DAD, while also including solvent evaporation and analyte reconstitution (theoretical preconcentration factor of 5) [151]. In fact, for E2 and EE2 the LOQ values in this research by Socas-Rodriguez et al. are better than those in the FPSE-HPLC-UV method, and then the performance of the method drops off significantly for E1, DES, and HEX.

Table 16: Comparison of LOD and LOQ values obtained with the developed FPSE(PTHF)-HPLC-UV method vs HF-LPME-HPLC-DAD/FD and Acetate$\mathrm{MeOH}-S P E-H P L C-D A D$ methods from the literature. N/A signifies that the compound is not included in the data.

\begin{tabular}{cc|c|c|c|c|}
\multirow{2}{*}{ Compound } & \multicolumn{2}{c}{ FPSE-HPLC-UV } & \multicolumn{2}{c}{$\begin{array}{c}\text { HF-LPME-HPLC- } \\
\text { DAD/FD }\end{array}$} & $\begin{array}{c}\text { Acetate-MeOH- } \\
\text { SPE- HPLC-DAD }\end{array}$ \\
\cline { 2 - 6 } & $\begin{array}{c}\text { LOD } \\
(\mathrm{ppb})\end{array}$ & $\begin{array}{c}\mathrm{LOQ} \\
(\mathrm{ppb})\end{array}$ & $\begin{array}{c}\mathrm{LOD} \\
(\mathrm{ppb})\end{array}$ & $\begin{array}{c}\mathrm{LOQ} \\
(\mathrm{ppb})\end{array}$ & MQL (ppb) \\
\hline BPA & 6.48 & 21.6 & $\mathrm{~N} / \mathrm{A}$ & $\mathrm{N} / \mathrm{A}$ & $\mathrm{N} / \mathrm{A}$ \\
\hline E2 & 1.59 & 5.29 & 0.81 & 2.7 & 630 \\
\hline EE2 & 3.65 & 12.2 & 1.7 & 5.7 & $\mathrm{~N} / \mathrm{A}$ \\
\hline E1 & 1.03 & 3.45 & 13.0 & 43.3 & 360 \\
\hline DES & 1.89 & 6.28 & 80.9 & 269 & 100 \\
\hline HEX & 1.19 & 3.95 & 13.2 & 44.0 & 340 \\
\hline
\end{tabular}


To illustrate that the LOD and LOQ values of the FPSE based method presented herein would be much better with this post-extraction solvent evaporation and analyte reconstitution, Fig. 90 shows a comparison of the magnitude of response of the analyses injected before and after. The solvent ( $350 \mu \mathrm{L}$ of ACN) was evaporated with a stream of nitrogen gas at room temperature, then the analytes were reconstituted to $50 \mu \mathrm{L} \mathrm{ACN}$. Evaporating the solvent such that none of it would be spilled (which would create loss of analytes) took 2.5 hours. While this technique increased the response for each compound significantly, the factor by which each response was increased was not 7 , which is the theoretical preconcentration factor in this case, and is not consistent for all analytes. These findings present clear evidence that this procedure should be avoided whenever possible because it takes a very long time and makes accurate quantitation practically impossible. 


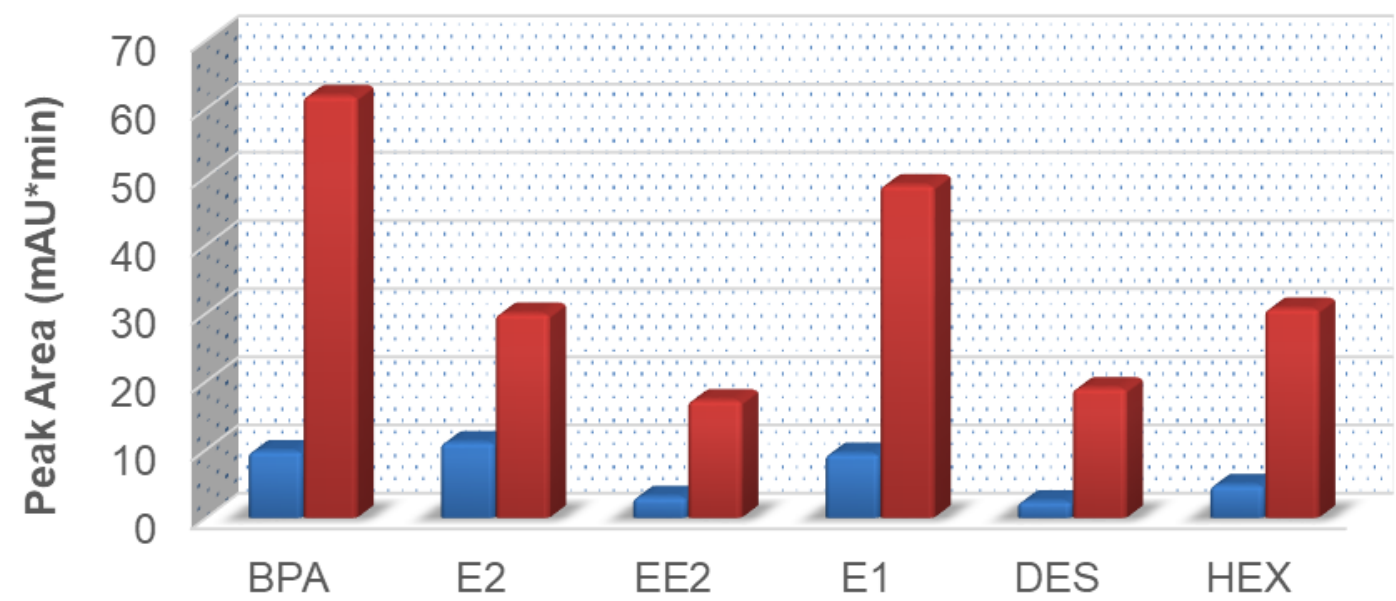

घBefore Evaporation/Reconstitution a After Evaporation/Reconstution

Figure 90: Illustration of experimental data comparison responses generated by analysis of EDCs in whole milk by the developed FPSE(PTHF)-HPLC-UV method before and after solvent evaporation and analyte reconstitution.

\subsubsection{Assessment of Fat Content Bias}

It is commonly hypothesized that one of reasons that whole milk is such a challenging sample matrix is the fat content. The fat in whole milk (approximately $4.3 \% \mathrm{v} / \mathrm{v}$ ) contributes to the difficulty of extracting small compounds such as EDCs as it contributes to the viscosity and forces protein precipitation and defatting for methodologies such as SPE. However, it is also believed that fat interacts with the analytes at the molecular level and the results presented in Fig. 91 are proof of that. Milk, being an animal product, contains most unsaturated fat, which can interact small organic compounds such as EDCs via London dispersion forces (induced dipole interactions) and dipole-induced dipole interactions. 


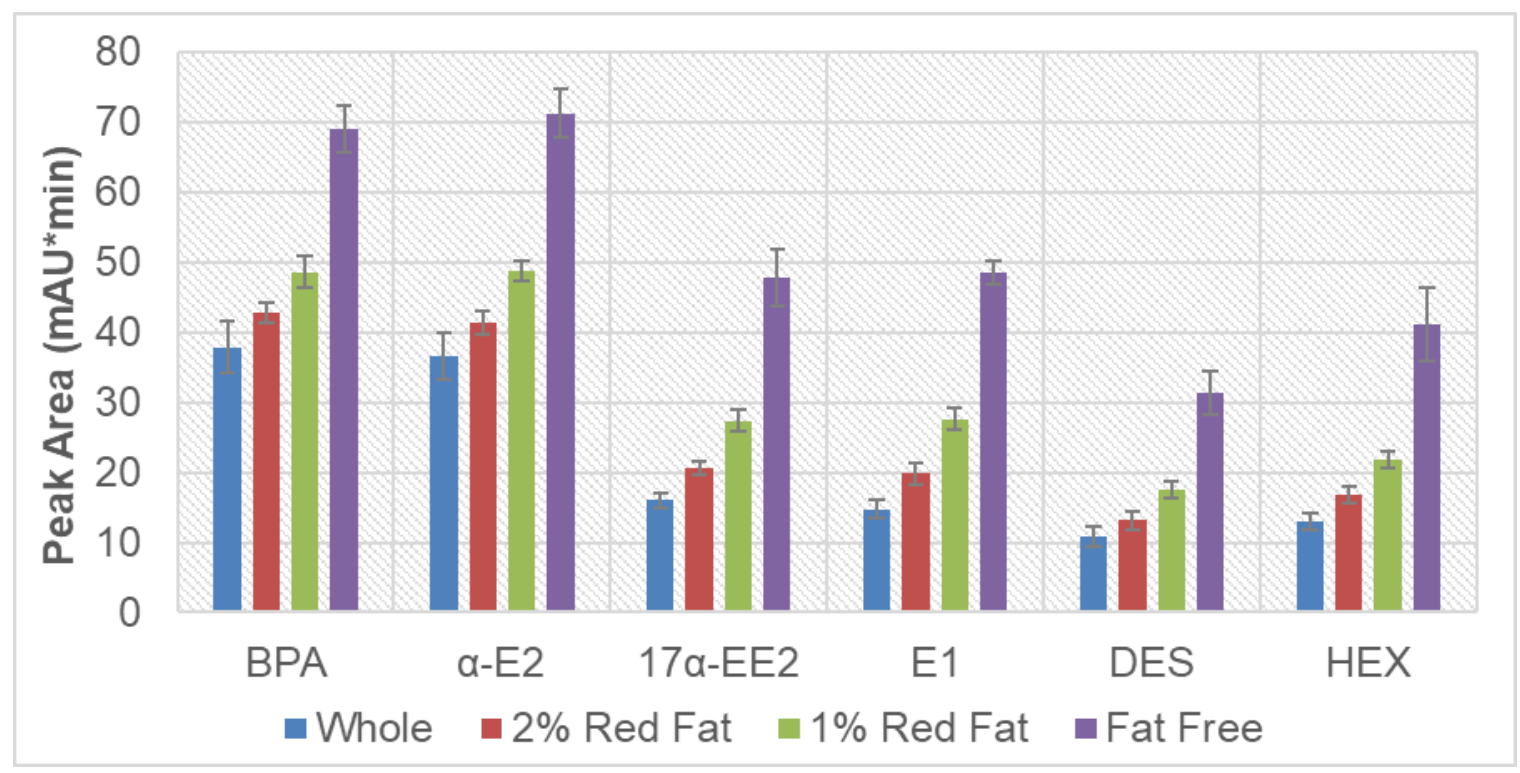

Figure 91: Experimental data of assessment of fat content effect on extraction sensitivity and reliability. Extraction efficiency of the developed FPSE(PTHF)HPLC-UV method is explored for whole milk, reduced fat milks ( $2 \%$ and $1 \%)$ and fat free milk $(<0.1 \%)$, respectively.

\subsubsection{Direct Analysis vs. Analysis After Protein Precipitation and Defatting}

At this point the FPSE(PTHF)-HPLC-UV method presented here has been proven to be more convenient and sensitive than those using conventional sample preparation techniques such as SPE, even when those are followed by solvent evaporation and analyte reconstitution. To determine whether this improvement in sensitivity is due primarily to the aforementioned advantages of sol-gel coatings such as fast mass transfer and porosity of the material or whether it is because analytes are lost in the sample preparation and analyte reconstitution process, the FPSE-HPLC-UV method was applied to a sample of milk after such a process. This was done with two different acidic solutions commonly used for this purpose, acetic acid and formic acid, and compared to extracting from whole milk directly. It can be seen in Fig. 92 that extracting 
directly is without question the better option and that most of the analytes were lost in the protein precipitation and defatting process. Responses were not recorded for BPA, E2, EE2 or DES after acetic acid protein precipitation and defatting because the added steps led to interferences that significantly affected the resulting chromatograms. Even if this hadn't happened, the responses for those compounds would not have been competitive with those from direct extraction. This is known because there was no evidence whatsoever of a high peak being masked.

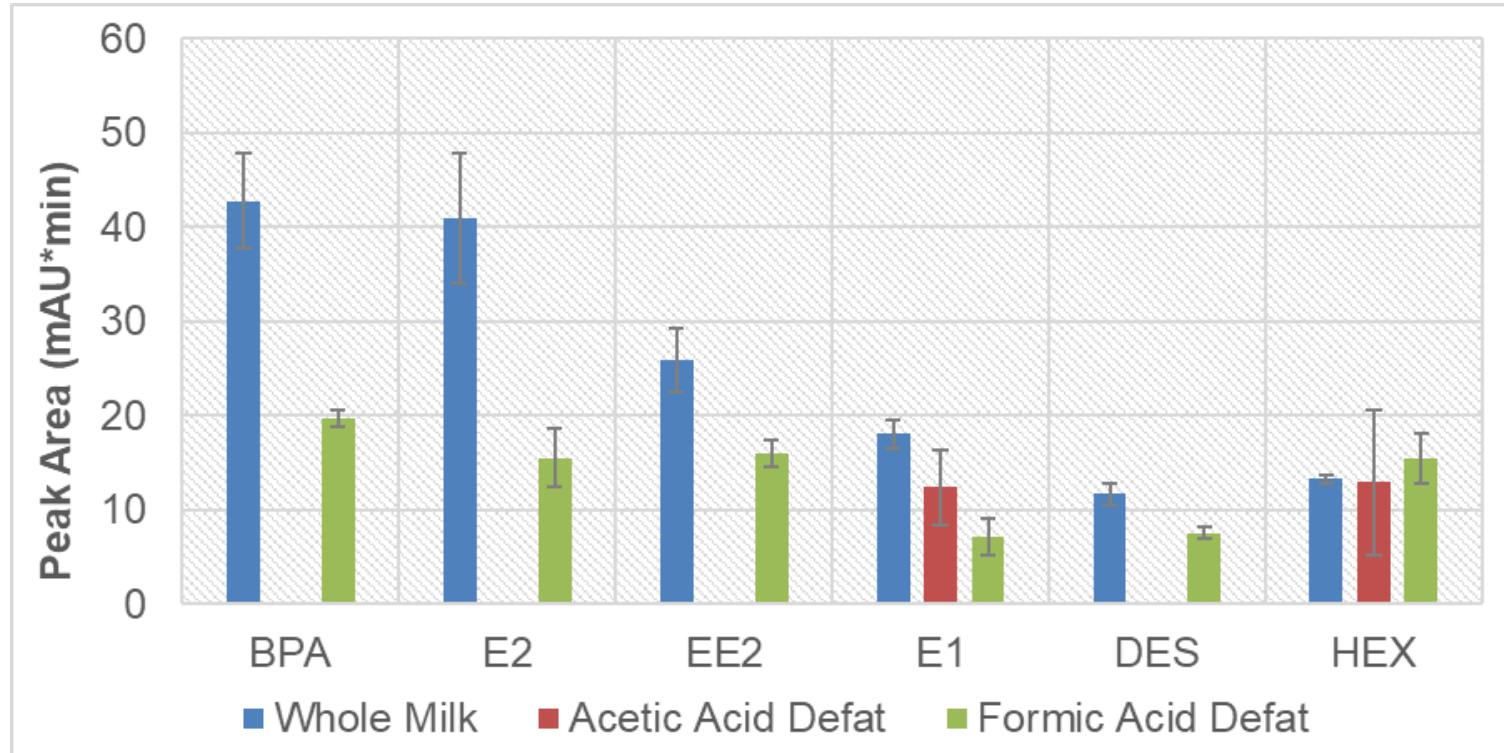

Figure 92: Graphical representation of analysis bias due to protein precipitation and defatting using the developed FPSE(PTHF)-HPLC-UV for analysis of 6 EDCs from whole milk.

\subsubsection{FPSE(PTHF)-HPLC-(QQQ)MS/MS}

When using UV detectors, or really any detector that is not a mass spectrometer, there can be uncertainty about whether the peaks seen in a chromatogram of analysis of complex matrices actually represent the compound assigned to them. 
This concern is augmented when the sample matrix is one as complex as whole milk. For example, in the above chromatogram, are those peaks truly given by the indicated compounds? Needing to answer this question, an HPLC(QQQ)MS/MS method was developed for the 6 EDCs included in the research project and the identity of each analyte was confirmed.

The chromatographic part of the HPLC-UV method was carried over with some minor modifications to HPLC-(QQQ)MS/MS method development. This was suitable because the same Zorbax C-18 Extend column was used. The (QQQ)MS/MS parameters were determined separately. Fig. 93 shows the resulting extracted ion chromatograms (EICs) of FPSE extraction from whole milk spiked to a concentration of BPA, E2, E1, DES, and HEX was $10 \mathrm{ppb}$ and the concentration of EE2 was $50 \mathrm{ppb}$. The injection volume was $20 \mu \mathrm{L}$ and the mass spectrometer mode was positive. For each analyte two transitions were monitored, along with the corresponding retention time. 

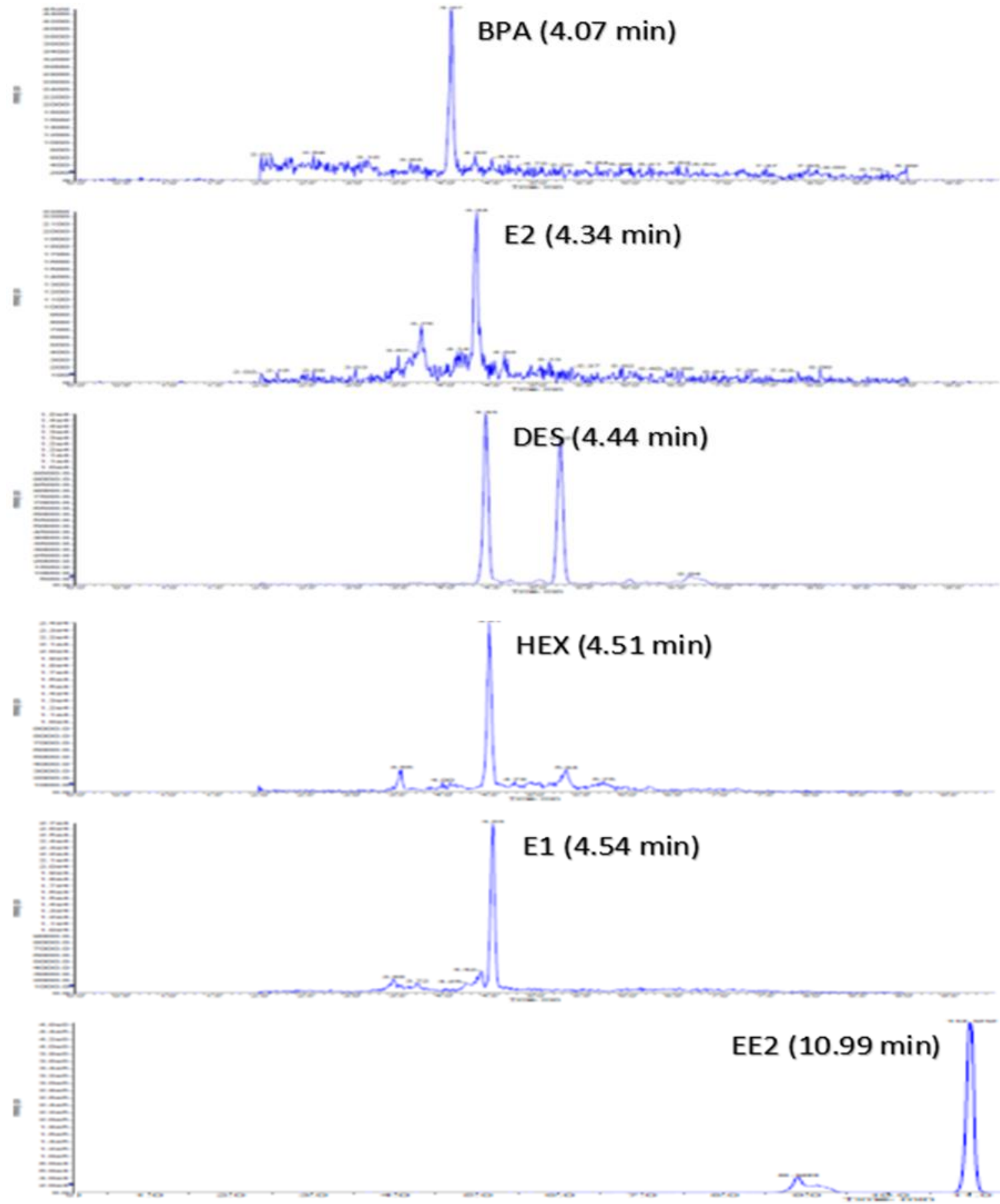

Figure 93: EICs of FPSE based analysis of whole milk spiked with the 6 pertinent EDCs. The concentration for BPA, E2, E1, DES, and HEX was 10 ppb of each in whole milk. The concentration of EE2 was $50 \mathrm{ppb}$. 
Sample preparation by FPSE was effortlessly coupled to this HPLC-

(QQQ)MS/MS method. The FPSE(PTHF)-HPLC-(QQQ)MS/MS method resulted in stupendous improvement on the already low sensitivity of the FPSE(PTHF)HPLC-UV method, as seen in Table 17. This table also compares the LOD values of the FPSE method coupled to tandem mass spectrometry with those from a recent ACN-SPE-HPLC-MS/MS method found in the literature [119] that was developed for various estrogenic EDCs, including E2, EE2, DES, HEX among its compounds. The SPE cartridge used was an Oasis HLB (Hydrophilic Lipophilic Balance) from Waters, which includes a state of the art stationary phase that combines lipophilic functionality for RP retention and hydrophilic functionality for retention of very polar analytes.

Table 17: Comparison of LOD and LOQ values determined with the developed FPSE(PTHF)-HPLC-UV and FPSE(PTHF)-HPLC-(QQQ)MS/MS methods and an ACN-SPE-HPLC-MS/MS methods from the literature. N/A: that compound not included in the data [119].

\begin{tabular}{|c|c|c|c|c|c|}
\hline \multirow{2}{*}{ Compound } & \multicolumn{2}{|c|}{ FPSE-HPLC-UV } & \multicolumn{2}{|c|}{ FPSE-HPLC-MS/MS } & \multirow{2}{*}{$\begin{array}{c}\text { ACN-SPE- } \\
\text { HPLC-MS/MS } \\
\text { LOD (ppb) }\end{array}$} \\
\hline & LOD (ppb) & LOQ (ppb) & LOD (ppb) & LOQ (ppb) & \\
\hline BPA & 6.48 & 21.6 & 0.693 & 2.31 & $\mathrm{~N} / \mathrm{A}$ \\
\hline E2 & 1.59 & 5.29 & 0.734 & 2.45 & 0.13 \\
\hline EE2 & 3.65 & 12.2 & 0.00602 & 0.0201 & 0.16 \\
\hline E1 & 1.03 & 3.45 & 0.091 & 0.302 & $N / A$ \\
\hline DES & 1.89 & 6.28 & 1.190 & 3.97 & 0.14 \\
\hline HEX & 1.19 & 3.95 & 0.114 & 0.379 & 0.12 \\
\hline
\end{tabular}


The sensitivity values reported by Theodoridis \& Kaklamanos for E2, EE2, DES and HEX are very impressive. As can be seen in Table 17, their reported values for LOD of E2 and DES are lower than those being reported here. This is not such a simple comparison, however. This SPE based method, as described in the corresponding article, made use of solvent evaporation and analyte reconstitution by dissolving a very large amount of eluent solvent $(3 \mathrm{~mL})$ and reconstituting in $100 \mu \mathrm{L}$ (theoretical preconcentration factor of 30 ) [119]. If this had not been done the corresponding LOD values would be much higher than those by the FPSE(PTHF)-(QQQ)MS/MS.

\subsubsection{Comparison of Procedure of Developed Method to SBSE and SPE Based Methods}

It is important to keep in mind the relative simplicity of the developed FPSE method being presented herein, in that it extracts from whole milk directly and produces the preceding figures of merit without solvent evaporation and analyte reconstitution. This is one of the best characteristics of FPSE methods in general, and even more so when the sample matrix is such a complex one such as whole milk. To put this in perspective, Fig. 94 consists of flowcharts comparing the procedures two recently published methods $[150,152]$ for analysis of milk to the one being introduced here. The two methods for which the procedures were summarized in the form of a flowchart use SBSE and SPE for sample preparation, respectively. 

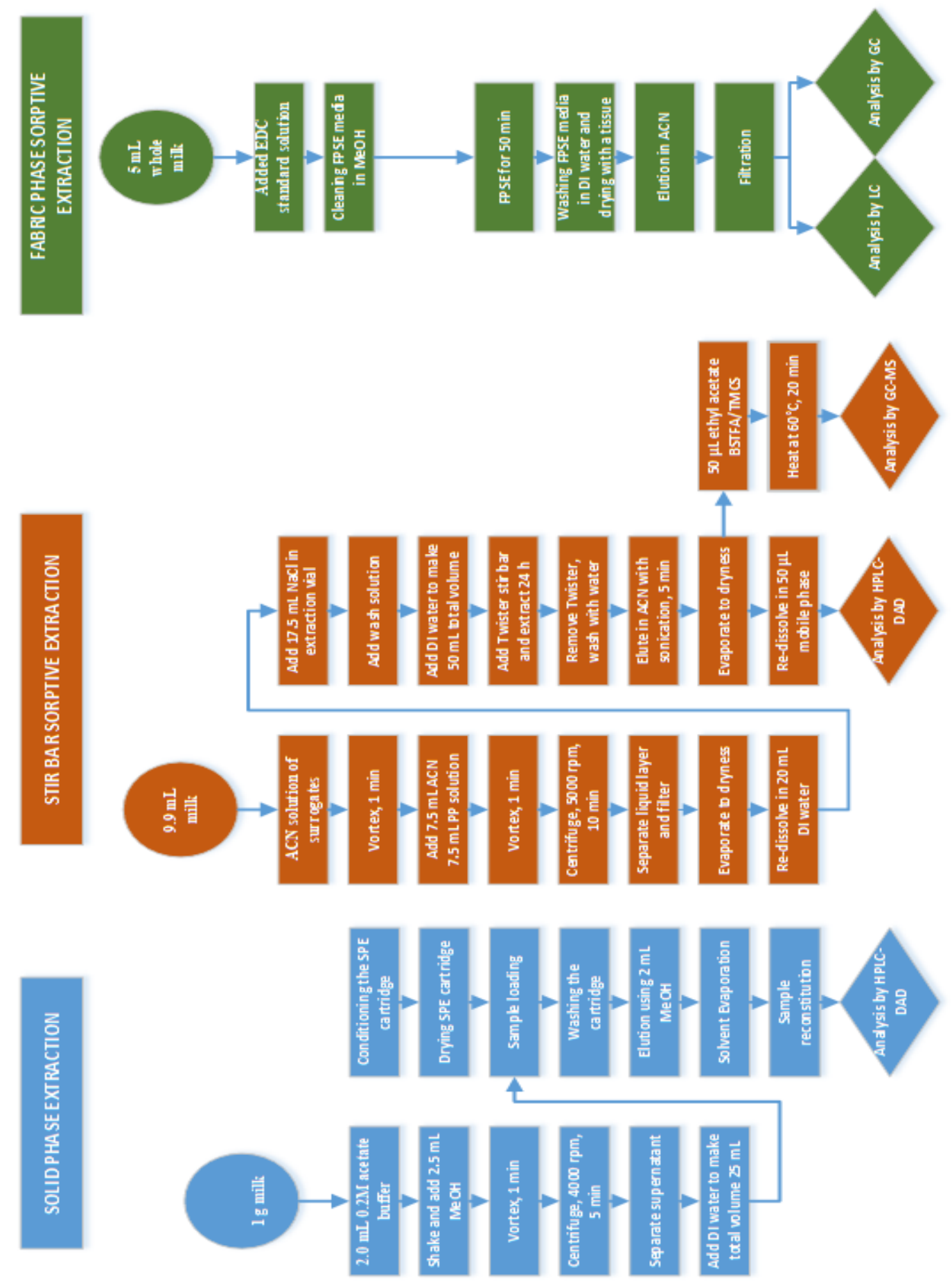

Figure 94: Flowcharts comparing the procedures of two recently published methods (SBSE and SPE used for sample preparation, respectively) for analysis of milk to the one being introduced here. 
5.7.10 Application of Developed Method to Spiked Urine and Whole Blood

Samples

This simple but fabulously effective FPSE method was subsequently used in a proof of concept study where it was applied to analysis of human urine and diluted whole human blood (1:4 whole blood:deionized water dilution). These matrices were spiked to a concentration of $200 \mathrm{ppb}$ for BPA, E2, and E1 and 400 ppb for EE2, DES, and HEX. No modification was done to the urine or the blood samples (other than the dilution) before sample preparation. The dilution of the blood was necessary to prevent clotting. While this is only a proof of concept study, it is clear from the chromatograms showed in Figs. 95 and 96 that the method is effective for analysis of EDCs in whole blood and urine.

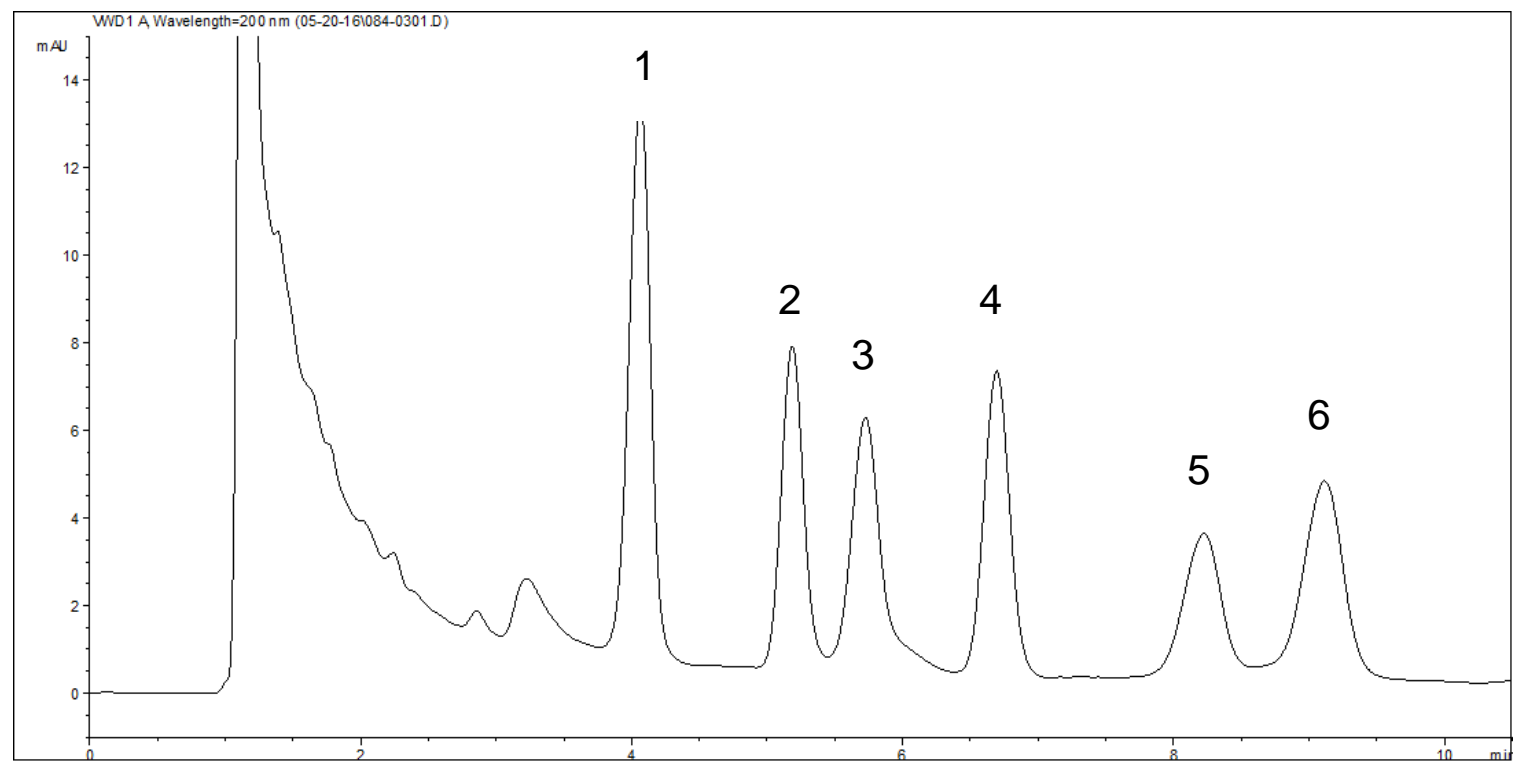

Figure 95: Chromatogram obtained from application of developed FPSE(PTHF)HPLC-UV method to spiked diluted whole blood. The peaks correspond to the EDCs as follows: (1) BPA, (2) E2, (3) EE2, (4) D1, (5) DES, (6) HEX 


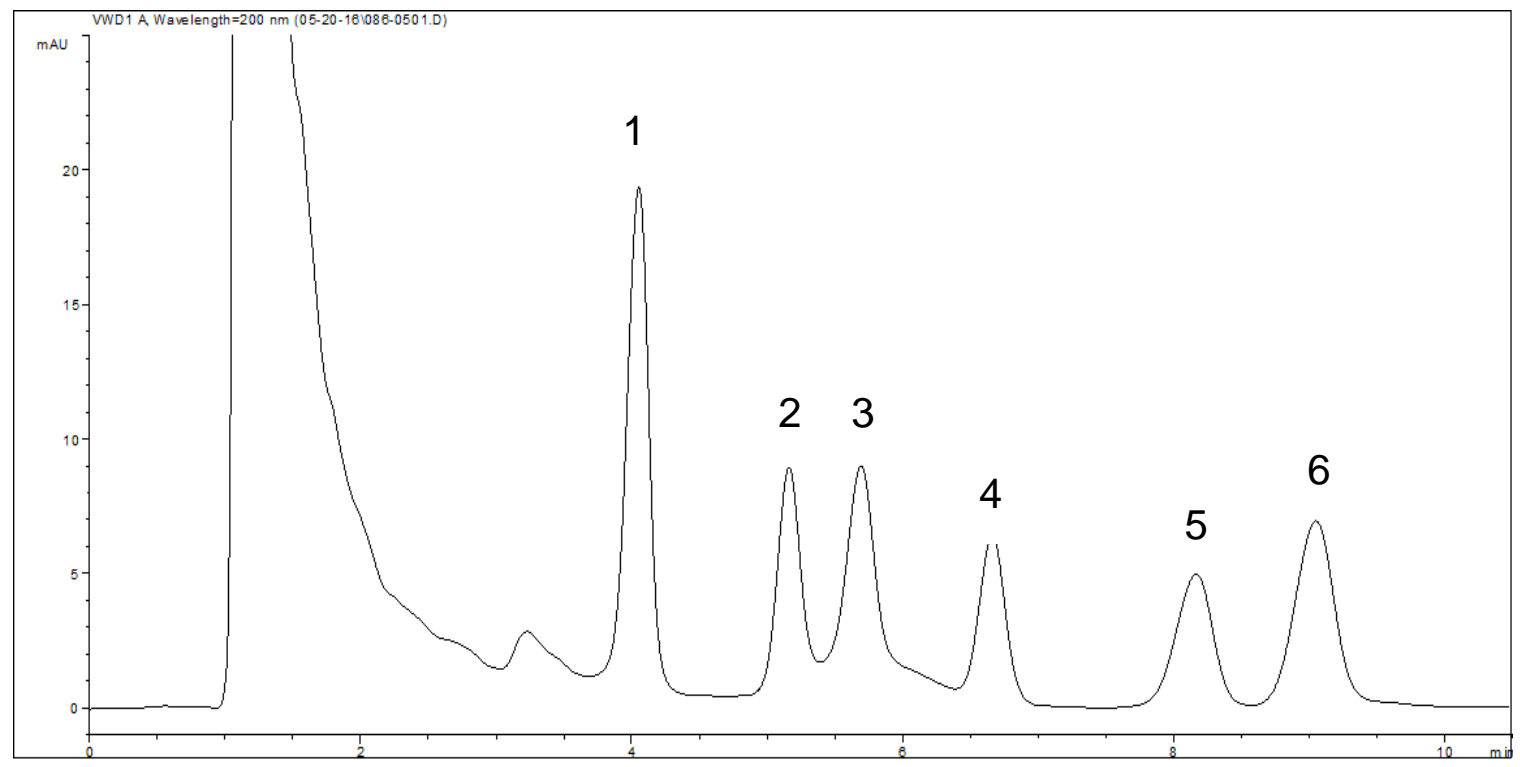

Figure 96: Chromatogram obtained from application of developed FPSE(PTHF)HPLC-UV method to spiked urine. The peaks correspond to the EDCs as follows: (1) BPA, (2) E2, (3) EE2, (4) D1, (5) DES, (6) HEX.

\subsection{FPSE Media: A Highly Effective Extraction and Storage Device}

Another investigated application of FPSE is extraction from water and retention of eight compounds of interest. The retention happens on the sol-gel sorbent of the FPSE media, in this case sol-gel PTHF, because the affinity of the analytes to the sorbent is strong enough. The compounds included in this study are 3,4and 3,5-dimethylphenol (compound class: substituted phenols); diphenylamine (DPA) and 2-nitrodiphenylamine (NDPA) (compound class: amines); benzophenone and t-chalcone (compound class: ketones); and phenanthrene and anthracene (compound class: PAHs).

This investigation was a priority primarily because storage of evidence is a very important and prevalent task performed by forensic laboratories. The experimental data presented subsequently serves as evidence that FPSE-PTHF 
media are a viable option for screening of samples due to its ability to simultaneously extract compounds of a wide variety of physicochemical properties. Additionally, FPSE media are equally effective for storage of these compounds and present a much safer, more convenient and cost-effective alternative, a fact made clear by the same data.

The first step in the procedure of this extraction and retention study was development of an HPLC-UV method for the mixture of the aforementioned 8 compounds mix. Once this task was accomplished, it was necessary to develop the sample preparation part of the method. This process was greatly expedited because methods had already been developed in house for PAHs, ketones, amines, and substituted phenols separately as part of other research projects. The effects of extraction and desorption parameters including extraction volume, extraction time, ionic strength, stirring rate, desorption time, and desorption solvent system on the extraction/desorption efficiency from the previously developed methods were then combined to be able to extract all eight compounds from water effectively.

The developed FPSE(PTHF)-HPLC-UV method was used for extraction of the eight selected compounds from a standard solution in deionized water. The concentration of each compound was $100 \mathrm{ppb}$. Thirty-nine replicate extractions (13 triplicate sets) were performed from $10 \mathrm{~mL}$ aliquots of the same aqueous solutions. Three of the FPSE media were back extracted from followed analysis in the HPLC-UV. Half of the remaining 36 FPSE media, at this point presumably 
retaining the analytes, were stored in glass vials in a freezer. The other 18 FPSE media were stored in a drawer without any temperature control. Two weeks after these extractions were done, six FPSE media were back extracted from, three from the group in the freezer and the other three from the drawer at ambient conditions. Each of the resulting solutions was run in the HPLC-UV with the same method and column used previously. The same procedure was followed to test the retention of the analytes by the FPSE media at 2, 4, 8, 6, 16, and 32 weeks from the time of the extraction. The experimental data from these two studies is shown in Figs. $97 \& 98$. An external standard was used each time with each data point to ensure lack of bias due to instrumental performance variations.

The effectiveness of the FPSE media as storage devices was tested both in frozen and ambient conditions because scientific specimens, in forensic laboratories and otherwise, are often stored in a frozen state to prevent a change in their composition as long as possible. This is a very common and effective practice. The possibility of storage of compounds on FPSE media was also tested without refrigeration because it would be even more advantageous to be able to store samples in ambient conditions. It would save energy and freezer space, not to mention that laboratories in some parts of the world don't have guaranteed consistent access to electricity. 


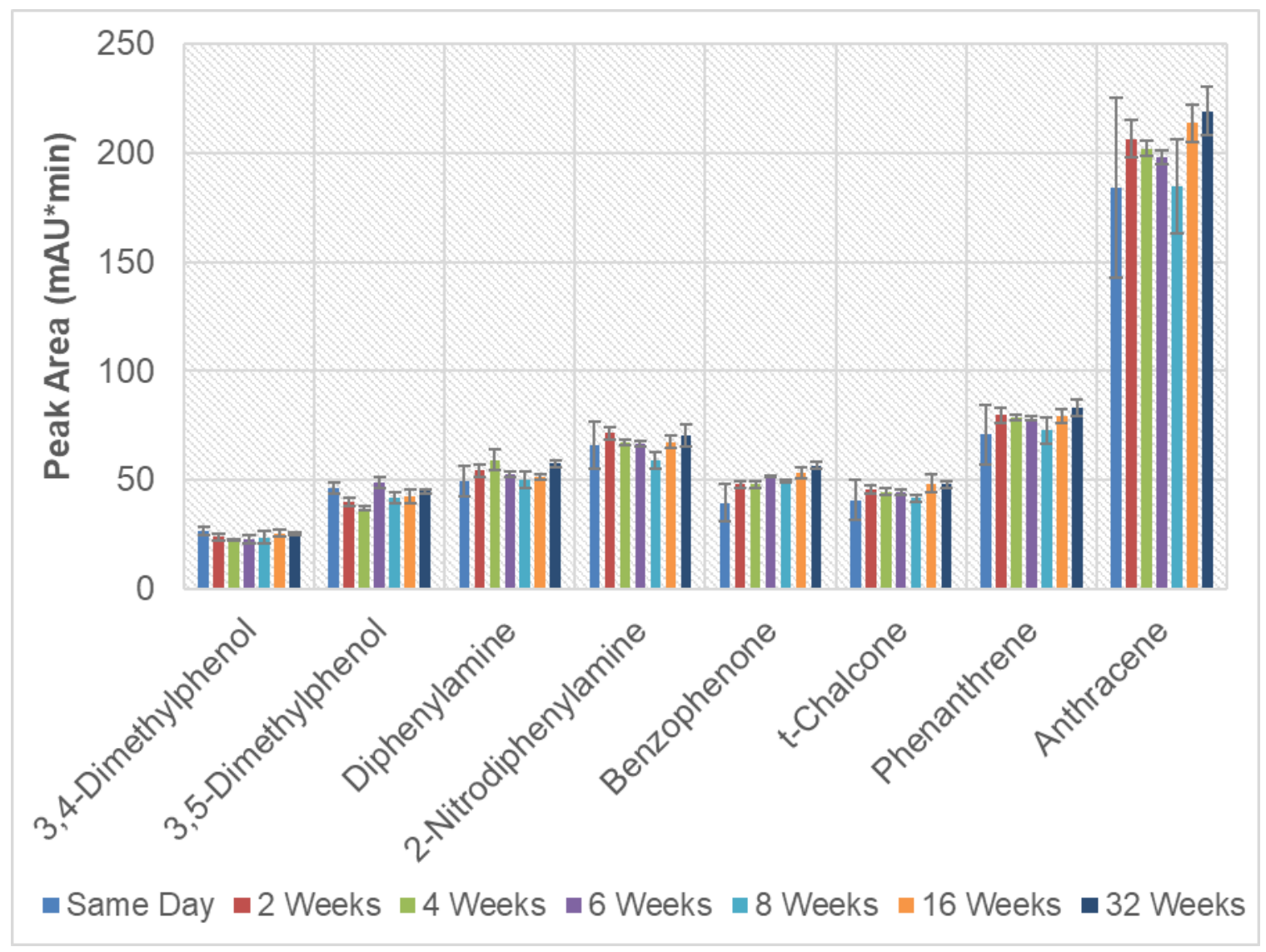

Figure 97: Experimental data showing compound specific results of the same day analysis of 8 relevant compounds in deionized water with the developed FPSE(PTHF)-HPLC-UV method vs the responses after frozen storage in the FPSE media sorbent for 2, 4, 6, 8, 16, and 32 weeks. 


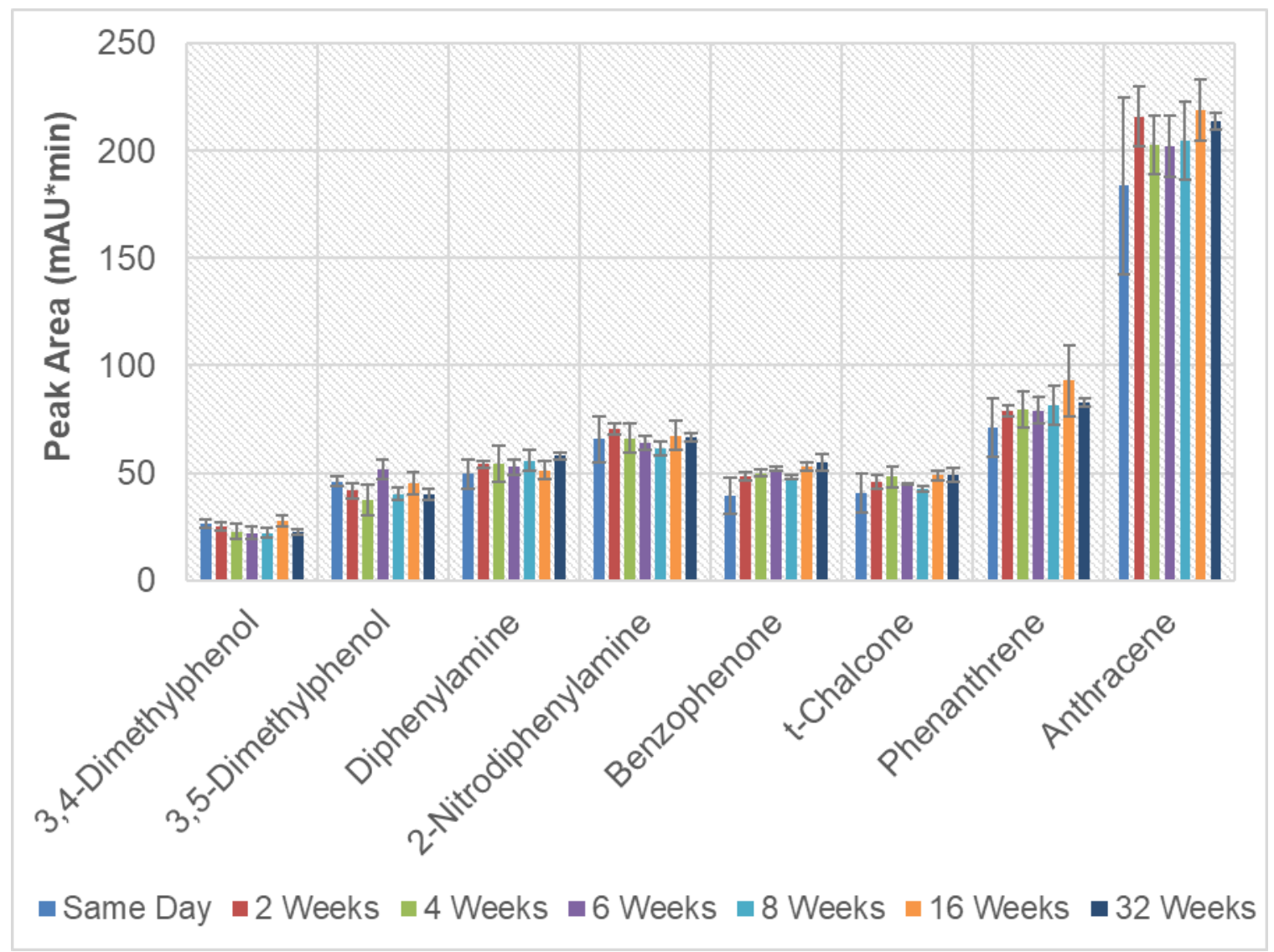

Figure 98: Experimental data showing compound specific results of the same day analysis of 8 relevant compounds in deionized water with the developed FPSE(PTHF)-HPLC-UV method vs the responses after storage at ambient temperature in the FPSE media sorbent for 2, 4, 6, 8, 16, and 32 weeks.

Figs. $97 \& 98$ clearly show that not only the method was effective for the eight selected compounds, the retention was superb. There is no loss of any of the compounds over the 32 week period from the FPSE media that were kept frozen nor from the ones at ambient conditions.

Figs. $99 \& 100$ provide experimental data that shows that the significance and implications of this application of FPSE media extends beyond cost effectiveness and convenience. There are samples that cannot be preserved in the form that they are obtained. The study that produced the data in Figs. $99 \& 100$ consisted 
of spiking a water sample from a local pond with the eight compounds for which the retention of the FPSE-PTHF media was tested. One hour after spiking, the developed FPSE based method was applied to three $10 \mathrm{~mL}$ aliquots of the solution. The rest of the spiked solution was divided in two equal parts and stored in glass containers. One was refrigerated (instead of being placed in the freezer to avoid freezing of the solution) and the other kept at ambient conditions. These solutions were analyzed again, in triplicate, after 1, 2, 3, 14, and 32 weeks of the pond water having been sampled and spiked.

The data in Figs. 99 \& 100 shows significant or complete loss of signal for the analytes by week 32 in both kinds of storage conditions. The ketones were lost in less time than the rest, as the signal for benzophenone and t-chalcone were either completely lost or nearly lost after just one week in storage in both kinds of storage conditions. The compounds in these solutions that were preserved the most were the substituted phenols (3,4- and 3,5-DMP) and amines (DPA and 2NDPA). There doesn't appear to be a significant loss of signal for these four analytes for the first three weeks in either storage condition, but three weeks is not a very long storage time in most situations. 


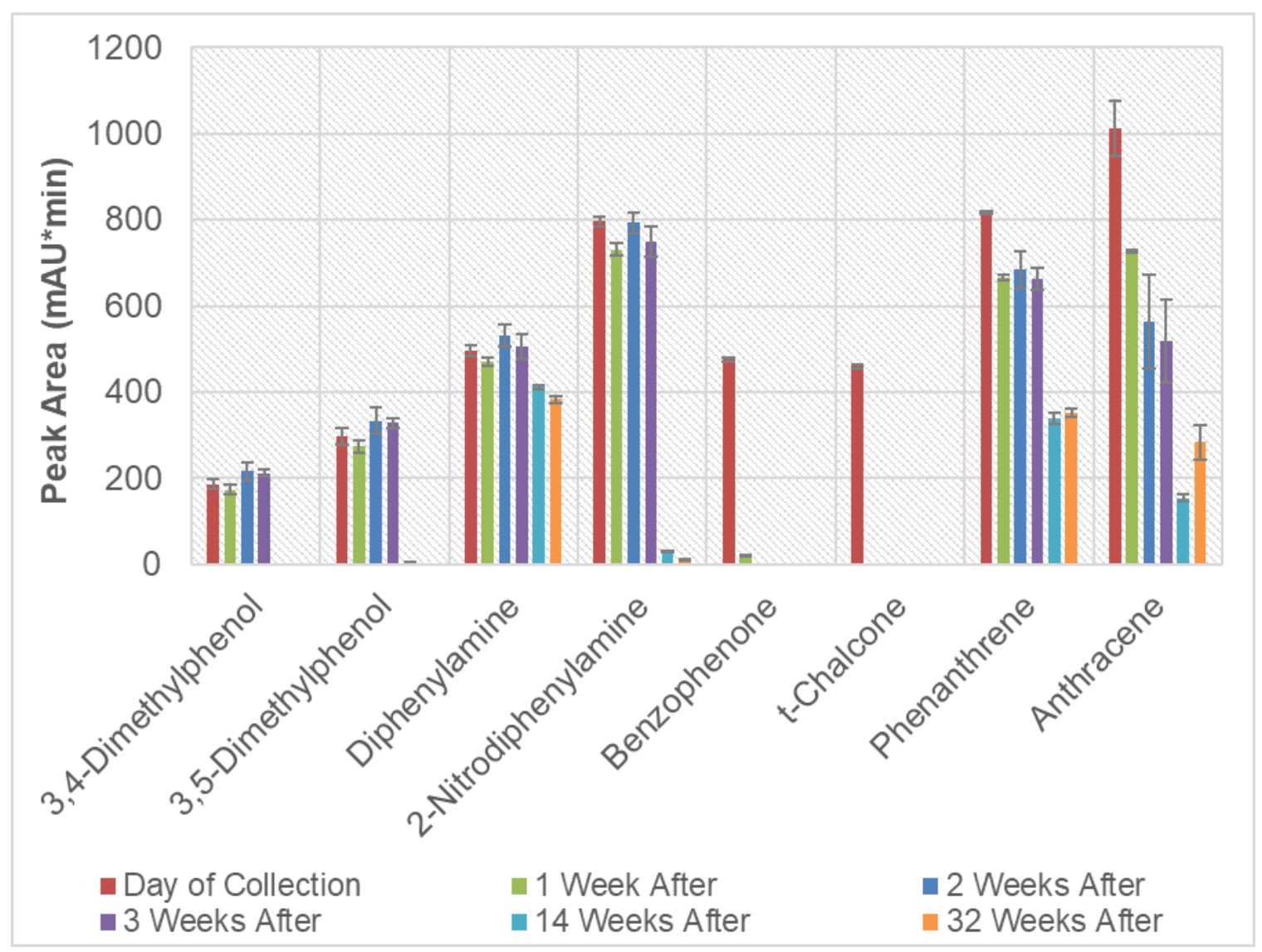

Figure 99: Experimental data showing compound specific results of the same day analysis of a spiked sample of pond water with the developed FPSE(PTHF)HPLC-UV method vs the responses after refrigerated storage of the solution for $1,2,3,14$, and 32 weeks. 


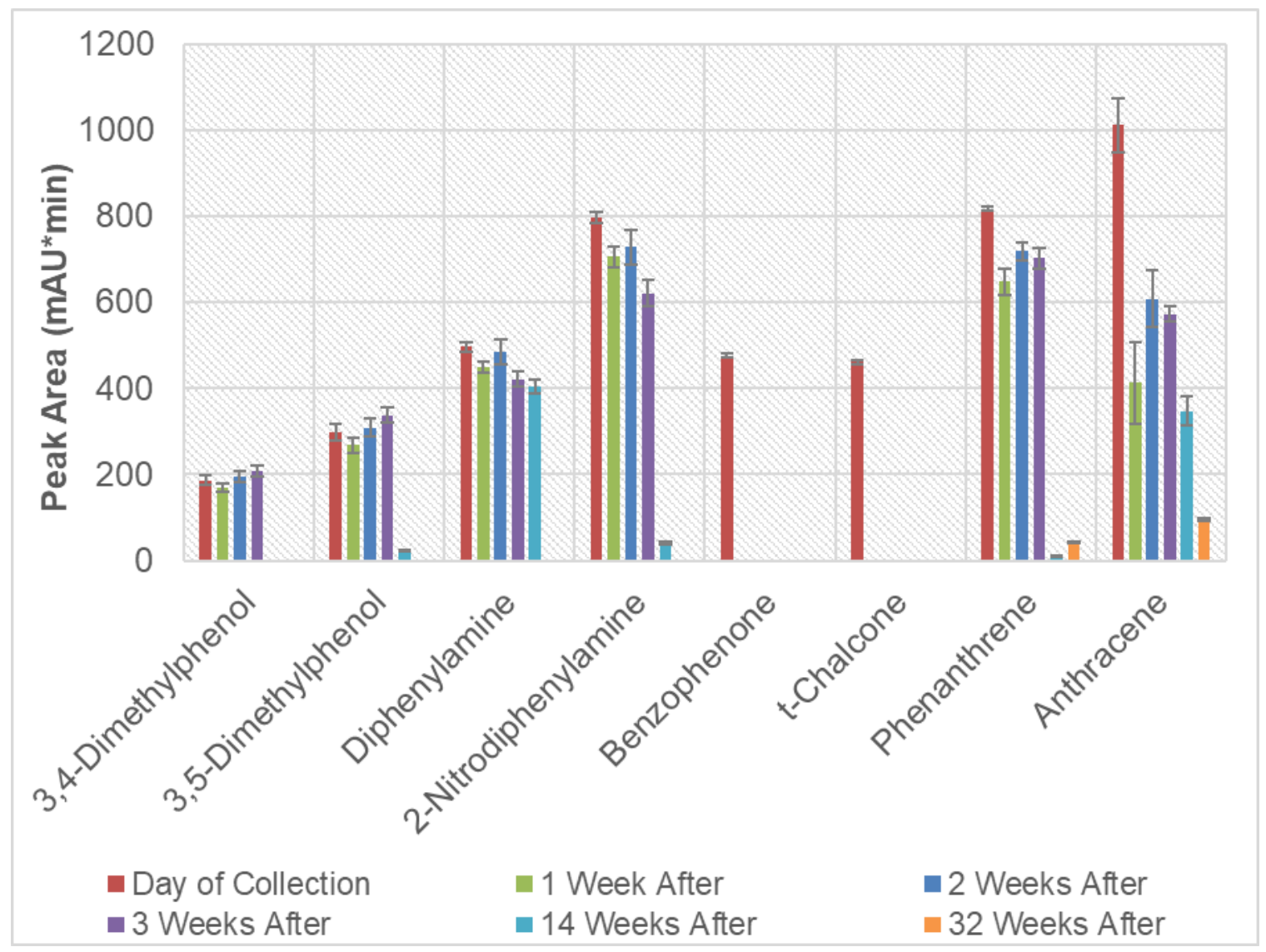

Figure 100: Experimental data showing compound specific results of the same day analysis of a spiked sample of pond water with the developed FPSE(PTHF)HPLC-UV method vs the responses after storage of the solution at ambient temperature for $1,2,3,14$, and 32 weeks.

The data presented in Figs. $97 \& 98$ cannot be interpreted in any other way than to say that FPSE(PTHF) media are a very effective option for screening purposes due to the ability to extract compounds with a wide range of physicochemical properties and if necessary store them. However, someone could possibly question whether this is achieved by the sol-gel sorbent on FPSE media or by cellulose fabrics themselves. To find an answer to this question, the same cellulose fabrics used as the substrate for FPSE(PTHF) media were tested for their ability to extract the same set of compounds and subsequently retain them. 
Everything was replicated just as it was done with the FPSE(PTHF), except that back extractions were done at 1, 2, 4, 8, 16, and 32 weeks, which deviates slightly from the plan followed with FPSE media. The resulting data is presented in Figs. 101 (storage in freezer) \& 102 (ambient storage).

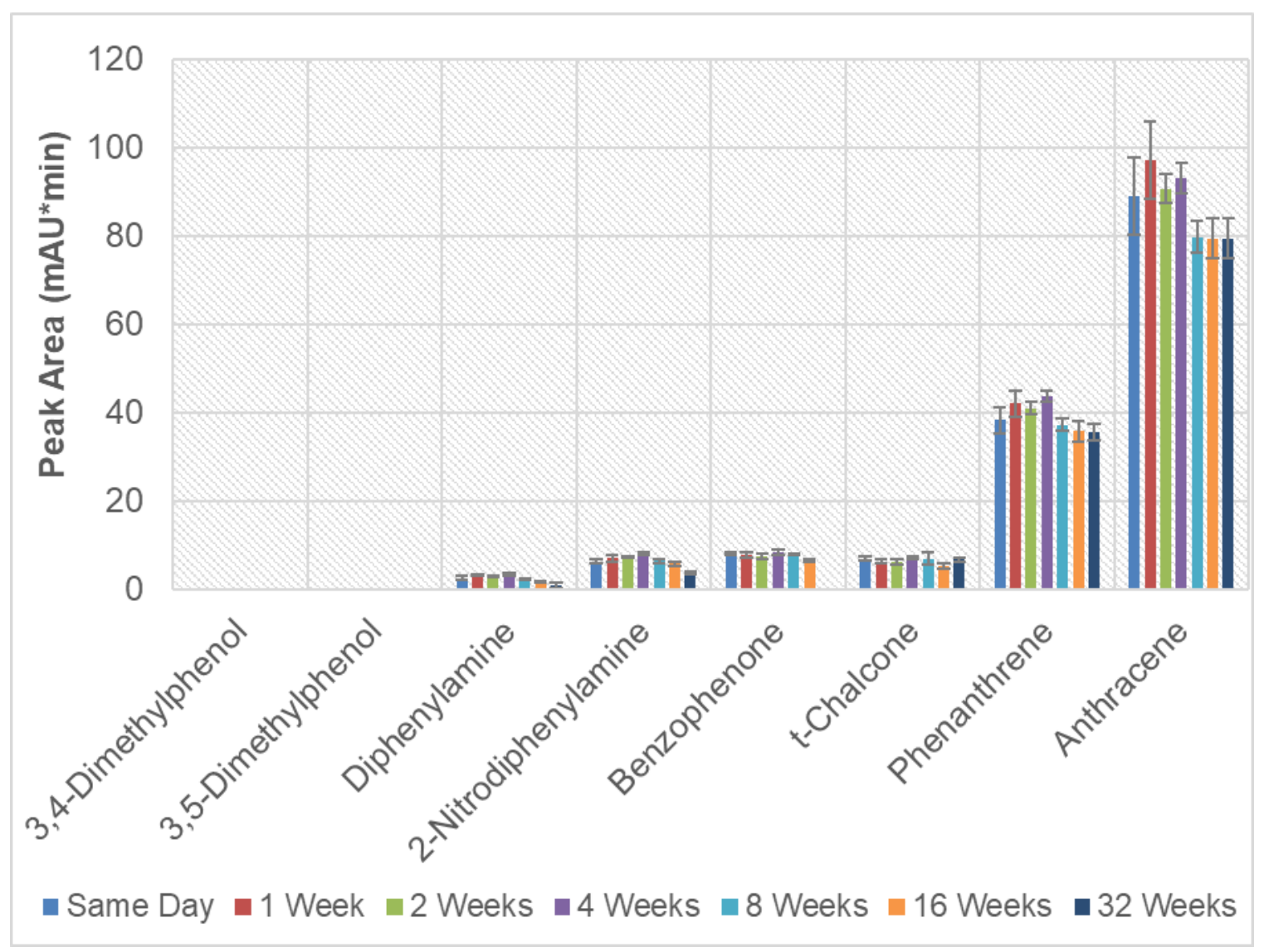

Figure 101: Experimental data showing results of analysis of 8 relevant compounds in deionized water vs the responses after refrigerated storage in the FPSE media sorbent for 1, 2, 4, 8, 16, and 32 weeks. Uncoated cellulose was used instead of FPSE-PTHF media. 


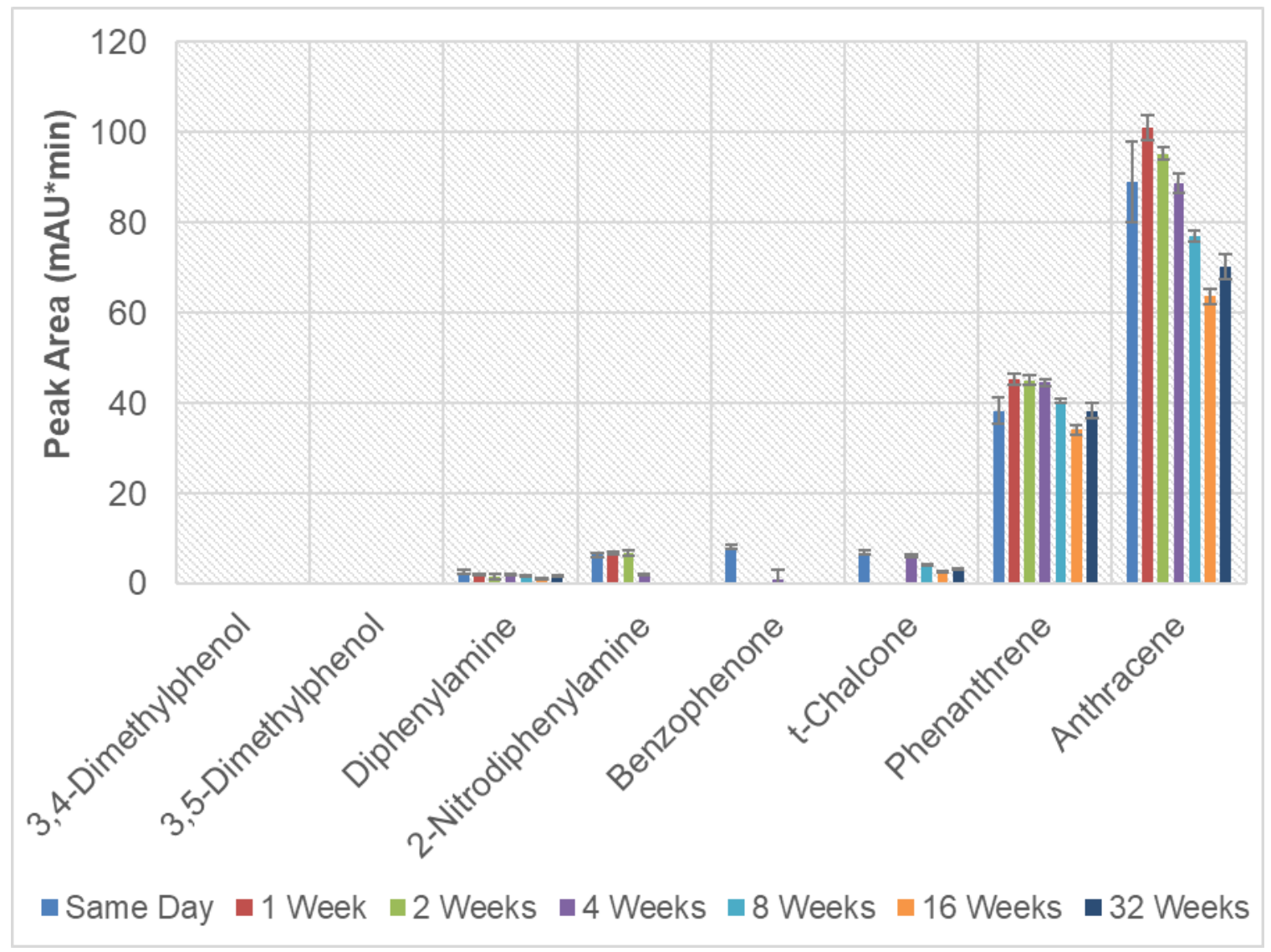

Figure 102: Experimental data showing results of analysis of 8 relevant compounds in deionized water vs the responses after storage at ambient temperature in the FPSE media sorbent for 2, 4, 6, 8, 16, and 32 weeks. Uncoated cellulose was used instead of FPSE-PTHF media.

The difference between the usefulness of cellulose without sol-gel sorbent and FPSE media is vast. The data in Figs. 101 \& 102 makes it obvious that bare cellulose is not nearly as effective as FPSE-PTHF media for extraction of any of the compounds in this study. The only two compounds for which responses large enough to be quantified reliably were produced were the PAHs, phenanthrene and anthracene. This happened because these chemicals are hydrophobic. The other six compounds, however, are slightly or highly polar and therefore not 
hydrophobic and the partition coefficient between the cellulose and water overwhelmingly favors the water.

The storage ability of the bare cellulose is also not comparable to that of the FPSE-PTHF media. DPA and phenanthrene we retained well enough but 2NDPA, benzophenone, $\mathrm{t}$-chalcone and anthracene were not as the signal decreased significantly for each of these compounds. The substituted phenols were not extracted enough to produce a signal so the retention of these by the cellulose could not be tested.

In conclusion, this study proves that FPSE media offer the ability to be used as forensic evidence collection and storage devices for analytes of a wide variety of chemical properties, therefore reducing the laborious, expensive and risky processes of collecting the sample from the field/crime scene and transporting it to the analytical laboratory before the sample preparation for instrumental analysis begins. It was also shown that this is made possible by the sol-gel sorbent of FPSE media, not the cellulose substrate.

\section{CONCLUSION}

The dangers of nitroaromatic explosives, substituted phenols, BTEX compounds, PAHs, and EDCs are well documented in this report and additionally in a plethora of published scholarly articles, websites, and textbooks. These classes of pollutants are all priorities in environmental and criminal forensics. Not only are they very harmful or even lethal to humans and wildlife, the abundance of them is high and for BTEX, PAHs, and estrogenic EDCs it is increasing. 
It is necessary to have the ability to detect and quantify compounds in these classes, preferably at levels lower than toxic thresholds so that measures can be taken to ensure safety of foods and environmental waters before people are injured or possibly even poisoned to death. Currently popular and long established methodologies, including those used in regulatory methods, simply fail to perform reliably to the extent necessary. SPME, for instance, will always be known as a revolutionary creation, and rightfully so, but is not effective in many situations and is better suited for air monitoring or headspace analysis. Whether it is traditional SPME or one of its many derivatives, the sorbent and coating technologies are outdated because they do not provide enough PCSA, sorbent loading capacity, ruggedness, or reliable sensitivity.

LLE has had remarkable staying power in analytical chemistry, but it does not conform to GAC principles and is quite laborious, inefficient, and time consuming. The microextraction derivatives of LLE, such as DLLME, do not present the same egregious violations of GAC principles as their predecessor but do face problems of sensitivity because of the small amount of extracting solvent. SPE is useful in some cases but requires lots of pretreatment to a viscous sample such as milk before it can be passed through a cartridge or even a disk. SPE procedures are long, tiresome and difficult and force solvent evaporation and analyte reconstitution. While solvent evaporation and subsequent reconstitution with a convenient organic solvent serves a specific purpose, it is yet another long and difficult step in the SPE-based analytical process and should be avoided whenever possible. 
Fortunately, there is now a better option for direct immersion extractions from even the most complex liquid matrices: FPSE. The data presented previously in this dissertation undoubtedly supports the deservedly grandiose claims hailing FPSE as a paradigm shift in sample preparation technology. FPSE provides the following characteristics which are known to be ideal for sample preparation: simplicity (reduces error); high efficiency and throughput (saves time); not overly laborious; makes it possible to perform procedure on several samples simultaneously; ability to extract without pretreatment of sample matrix; high PCF and therefore sensitivity; portability; adherence to GAC principles; low cost of production that creates accessibility to more people; physical, solvent and chemical stability given by the rugged nature of sol-gel materials; and bonding of the sorbent to the substrate. Moreover, there has not been any automation of FPSE, but once it becomes commercially available there are no impediments to its immediate development.

Experimental data presented here also proves that FPSE media are not only efficient and effective for extraction from a wide variety of sample matrices, they are equally useful for storage of analytes even at ambient conditions. This may be adopted by forensic laboratories to save millions of dollars annually while ensuring the chemical profile of evidence samples.

This dissertation includes several independent studies but still delivers one message above all others, and it does so emphatically: FPSE ought to be considered in all analytical laboratories worldwide because while it may not 
always be the best available option, it certainly is the best choice more often than any other sample preparation technology that has been published and thoroughly investigated to date. 


\section{REFERENCES}

[1] U. Lewin, J. Efer, W. Engewald, High-performance liquid chromatographic analysis with electrochemical detection for residues of explosives in water samples around a former ammunition plant, J. Chromatogr. A. 730 (1996) 161-167. http://ac.els-cdn.com.ezproxy.fiu.edu/0021967395010777/1-s2.00021967395010777-main.pdf?_tid=52a0a20e-5f73-11e7-87cb00000aab0f02\&acdnat $=1499033637$ f55bd48f8f4c649ce35c273a7a38174 4.

[2] F. Monteil-Rivera, C. Beaulieu, J. Hawari, Use of solid-phase microextraction/gas chromatography-electron capture detection for the determination of energetic chemicals in marine samples, J. Chromatogr. A. 1066 (2005) 177-187. doi:10.1016/j.chroma.2005.01.049.

[3] J. Yinon, Forensic and environmental detection of explosives, John Wiley, 1999.

[4] J. Yinon, Toxicity and metabolism of explosives, CRC Press, 1990.

[5] EPA, Drinking Water Health Advisory for TNT, Washington, DC, 1989.

[6] K.-H. Chang, T.-F. Chen, H.-C. Huang, Estimation of biogenic volatile organic compounds emissions in subtropical island-Taiwan, Sci. Total Environ. 346 (2005) 184-199. http://ac.elscdn.com.ezproxy.fiu.edu/S0048969704007818/1-s2.0S0048969704007818-main.pdf?_tid=514ece80-5f96-11e7-983400000aab0f26\&acdnat=1499048667_9ea3d6fea1bb4f6657bb18c363b0ab be.

[7] N. Yassaa, E. Brancaleoni, M. Frattoni, P. Ciccioli, Isomeric analysis of BTEXs in the atmosphere using ?-cyclodextrin capillary chromatography coupled with thermal desorption and mass spectrometry, Chemosphere. 63 (2006) 502-508. doi:10.1016/j.chemosphere.2005.08.010.

[8] CDC, Fourth National Report on Human Exposure to Environmental Chemicals, 2015.

[9] I. V. Gruzdev, I.M. Kuzivanov, I.G. Zenkevich, B.M. Kondratenok, Determination of methyl-substituted phenols in water by gas chromatography with preliminary iodination, J. Anal. Chem. 68 (2013) 161169. doi:10.1134/S106193481302010X.

[10] A. Kabir, R. Mesa, J. Jurmain, K. Furton, Fabric Phase Sorptive Extraction Explained, Separations. 4 (2017) 21. doi:10.3390/separations4020021.

[11] W.-H. Zhang, D. Zhang, R.-J. Zhang, F. Xia, Y.-F. Liu, Flow injection analysis of volatile phenols in environmental water samples using 
CdTe/ZnSe nanocrystals as a fluorescent probe, Anal. Bioanal. Chem. 402 (2012) 895-901. doi:10.1007/s00216-011-5477-2.

[12] R.W. Giese, Measurement of endogenous estrogens: analytical challenges and recent advances, J. Chromatogr. A. 1000 (2003) 401-412. doi:10.1016/S0021-9673(03)00306-6.

[13] EPA, EPA Chemistry Dashboard Bisphenol A, (n.d.). https://comptox.epa.gov/dashboard/DTXSID7020182.

[14] A. Kabir, K.G. Furton, Fabric Phase Sorptive Extractors, 61/787,910, 2013. https://www.google.com/patents/US20140274660.

[15] A. Kabir, H. Holness, K.G. Furton, J.R. Almirall, Recent advances in microsample preparation with forensic applications, TrAC - Trends Anal. Chem. 45 (2013) 264-279. doi:10.1016/j.trac.2012.11.013.

[16] A. Kabir, C. Hamlet, A. Malik, Parts per quadrillion level ultra-trace determination of polar and nonpolar compounds via solvent-free capillary microextraction on surface-bonded sol-gel polytetrahydrofuran coating and gas chromatography-flame ionization detection, J. Chromatogr. A. 1047 (2004) 1-13. doi:10.1016/j.chroma.2004.06.078.

[17] S. Armenta, S. Garrigues, M. de la Guardia, Green Analytical Chemistry, TrAC - Trends Anal. Chem. 27 (2008) 497-511. doi:10.1016/j.trac.2008.05.003.

[18] C. Pin, J. Rodriguez, Separation Methods Based on Liquid-Liquid Extraction, Extraction Chromatography, and Other Miscellaneous Solid Phase Extraction Processes, in: Treatise on Geochemistry, 2014: pp. 147170. doi:10.1016/B978-0-08-095975-7.01409-1.

[19] D. Harvey, Liquid-Liquid Extractions - Chemistry LibreTexts, (2017). https://chem.libretexts.org/Textbook_Maps/Analytical_Chemistry_Textbook _Maps/Map:_Analytical_Chemistry_2.0_(Harvey)/07:_Collecting_and_Prep aring_Samples/7.7:_Liquid-Liquid_Extractions.

[20] J. Stevens, QuEChERS 101: The Basics and Beyond, (n.d.). https://www.agilent.com/cs/library/eseminars/Public/QuEChERS_101- The Basics and Beyond.pdf.

[21] F.R. Mansour, N.D. Danielson, Solidification of floating organic droplet in dispersive liquid-liquid microextraction as a green analytical tool, (2017). doi:10.1016/j.talanta.2017.03.084.

[22] J. Pawliszyn, Sampling and Sample Preparation for Field and Laboratory: Fundamentals and New Directions in Sample Preparation, in: Compr. Anal. Chem., ElsevierScience, Amsterdam, Netherlands, 2002.

[23] Y. He, H.K. Lee, Liquid-Phase Microextraction in a Single Drop of Organic 
Solvent by Using a Conventional Microsyringe, Anal. Chem. 69 (1997) 4634-4640. doi:10.1021/ac970242q.

[24] ¿ Fatemeh, S. Mirnaghi, K. Gor, A. Rodriguez-Lafuente, E. Boyacı, B. Bojko, J. Pawliszyn, Microextraction versus exhaustive extraction approaches for simultaneous analysis of compounds in wide range of polarity, J. Chromatogr. A. 1316 (2013) 37-43. doi:10.1016/j.chroma.2013.09.084.

[25] M. Rezaee, Y. Assadi, M.-R. Milani Hosseini, E. Aghaee, F. Ahmadi, S. Berijani, Determination of organic compounds in water using dispersive liquid?liquid microextraction, J. Chromatogr. A. 1116 (2006) 1-9. doi:10.1016/j.chroma.2006.03.007.

[26] M. Rezaee, Y. Yamini, M. Faraji, Evolution of dispersive liquid-liquid microextraction method, J. Chromatogr. A. 1217 (2010) 2342-2357. doi:10.1016/j.chroma.2009.11.088.

[27] A. V Herrera-Herrera, M. Asensio-Ramos, J. Hernández-Borges, M. Ngel Rodríguez-Delgado, Dispersive liquid-liquid microextraction for determination of organic analytes, Trends Anal. Chem. 29 (2010) 728-751. doi:10.1016/j.trac.2010.03.016.

[28] D. Barcelo, M.-C. Hennion, Trace determination of pesticides and their degradation products in water, First, Elsevier Science, Amsterdam, The Netherlands, 1997.

[29] M.-C. Hennio, Solid-phase extraction: method development, sorbents, and coupling with liquid chromatography, J. Chromatogr. A. 856 (1999) 3-54. doi:10.1016/S0021-9673(99)00832-8.

[30] M.-C. Hennion, C. Cau-Dit-Coumes, V. Picho, Trace analysis of polar organic pollutants in aqueous samples Tools for the rapid prediction and optimisation of the solid-phase extraction parameters, J. Chromatogr. A. 823 (1998) 147-161. http://ac.elscdn.com.ezproxy.fiu.edu/S0021967398004798/1-s2.0S0021967398004798-main.pdf?_tid=95e607d0-55c7-11e7-a18600000aacb35d\&acdnat=1497970316_998ad7c52a52b1deaeff8dd344a51c $\mathrm{db}$ (accessed June 20, 2017).

[31] C.F. Poole, A.D. Gunatilleka, R. Sethuraman, Contributions of theory to method development in solid-phase extraction, J. Chromatogr. A. 885 (2000) 17-39. doi:10.1016/S0021-9673(00)00224-7.

[32] D. Stevenson, Molecular imprinted polymers for solid-phase extraction, TrAC Trends Anal. Chem. 18 (1999) 154-158. doi:10.1016/S01659936(98)00094-6.

[33] N. Masqué, R.M. Marcé, F. Borrull, New polymeric and other types of 
sorbents for solid-phase extraction of polar organic micropollutants from environmental water, TrAC - Trends Anal. Chem. 17 (1998) 384-394. doi:10.1016/S0165-9936(98)00019-3.

[34] Supelclean ${ }^{\mathrm{TM}}$ ENVI and Supelclean ${ }^{\mathrm{TM}}$ SPE Cartridges - Solid Phase Extraction (SPE) | Sigma-Aldrich, (n.d.).

http://www.sigmaaldrich.com/analytical-chromatography/analyticalproducts.html?TablePage=9645027 (accessed June 21, 2017).

[35] H. Lingeman, S.J.F. Hoekstra-Oussoren, Particle-loaded membranes for sample concentration and/or cleanup in bioanalysis, J. Chromatogr. B Biomed. Appl. 689 (1997) 221-237. doi:10.1016/S0378-4347(96)00363-5.

[36] D.F. Hagen, C.G. Markell, G.A. Schmitt, D.D. Blevins, Membrane approach to solid-phase extractions, Anal. Chim. Acta. 236 (1990) 157-164. doi:10.1016/S0003-2670(00)83309-7.

[37] $3 \mathrm{M}$ Empore ${ }^{\mathrm{TM}}$ SPE Cartridges | Sigma-Aldrich, (n.d.). http://www.sigmaaldrich.com/analytical-chromatography/samplepreparation/spe/3m-empore/cartridges.html (accessed June 21, 2017).

[38] M.L. Mayer, C.F. Poole, Identification of the procedural steps that affect recovery of semi-volatile compounds by solid-phase extraction using cartridge and particle-loaded membrane (disk) devices, Anal. Chim. Acta. 294 (1994) 113-126. doi:10.1016/0003-2670(94)80186-X.

[39] E.M. (Earl M. Thurman, M.S. Mills, Solid-phase extraction : principles and practice, Wiley, 1998.

[40] N.J.K. Simpson, Solid-phase extraction : principles, techniques, and applications, Marcel Dekker, 2000. https://www.crcpress.com/Solid-PhaseExtraction-Principles-Techniques-andApplications/Simpson/p/book/9780824700218 (accessed June 21, 2017).

[41] E.R. Brouwer, S. Kofman, U.A.T. Brinkman, Selected procedures for the monitoring of polar pesticides and related microcontaminants in aquatic samples, J. Chromatogr. A. 703 (1995) 167-190. doi:10.1016/00219673(94)01237-9.

[42] J.S. Fritz, P.J. Dumont, L.W. Schmidt, Methods and materials for solidphase extraction, J. Chromatogr. A. 691 (1995) 133-140. doi:10.1016/0021-9673(94)00756-Y.

[43] A.R. Fernandez-Alba, A. Agüera, M. Contreras, G. Peñuela, I. Ferrer, D. Barceló, Comparison of various sample handling and analytical procedures for the monitoring of pesticides and metabolites in ground waters, J. Chromatogr. A. 823 (1998) 35-47. doi:10.1016/S0021-9673(98)00439-7.

[44] D. Barcelo, M.C. Hennion, On-line sample handling strategies next term for the trace-level determination of pesticides and their degradation products 
in environmental waters, Anal. Chim. Acta. 318 (1995) 1-41. doi:10.1016/0003-2670(95)00423-8.

[45] J. Pawliszyn, C.L. Arthur, Solid Phase Microextraction with Thermal Desorption Using Fused Silica Optical Fibers, Anal. Cham. 62 (1990) 2145-2148. http://pubs.acs.org/doi/pdf/10.1021/ac00218a019 (accessed June 11, 2017).

[46] S. Aldrich, Bioanalysis with SPME, (n.d.). http://www.sigmaaldrich.com/technical-documents/articles/reporterus/bioanalysis-with-spme.html.

[47] É.A. Souza-Silva, R. Jiang, A. Rodríguez-Lafuente, E. Gionfriddo, J. Pawliszyn, A critical review of the state of the art of solid-phase microextraction of complex matrices I. Environmental analysis, TrAC Trends Anal. Chem. 71 (2015). doi:10.1016/j.trac.2015.04.016.

[48] L.G. Blomberg, Current aspects of stationary phase immobilization in open tubular column chromatography, J. Microcolumn Sep. 2 (1990) 62-68. doi:10.1002/mcs.1220020204.

[49] E. Baltussen, P. Sandra, F. David, C. Cramers, Stir bar sorptive extraction (SBSE), a novel extraction technique for aqueous samples: Theory and principles, J. Microcolumn Sep. 11 (1999) 737-747. doi:10.1002/(SICI)1520-667X(1999)11:10<737::AID-MCS7>3.0.CO;2-4.

[50] E. Baltussen, H. Janssen, P. Sandra, C. a Cramers, A Novel Type of LiquidLiquid Extraction for the Preconcentration of Organic Micropollutants from Aqueous Samples: Application to the Analysis of PAH ' $s$ and OCP ' $s$ in Water, HRC J. High Resolut. Chromatogr. 20 (1997) 395-399.

[51] E. Baltussen, H.-G. Janssen, P. Sandra, C.A. Cramers, A new method for sorptive enrichment of gaseous samples: Application in air analysis and natural gas characterization, HRC J. High Resolut. Chromatogr. 20 (1997) 385-393.

[52] F. David, P. Sandra, Stir bar sorptive extraction for trace analysis, J. Chromatogr. A. 1152 (2007) 54-69. doi:10.1016/j.chroma.2007.01.032.

[53] I. Bruheim, X. Liu, J. Pawliszyn, Thin-Film Microextraction, Anal. Chem. 75 (2003) 1002-1010. doi:10.1021/ac026162q.

[54] S. Bigham, J. Medlar, A. Kabir, C. Shende, A. Alli, A. Malik, Sol-gel capillary microextraction, Anal. Chem. 74 (2002) 752-761. doi:10.1021/ac0109523.

[55] E. Karageorgou, N. Manousi, V. Samanidou, A. Kabir, K.G. Furton, Fabric phase sorptive extraction for the fast isolation of sulfonamides residues from raw milk followed by high performance liquid chromatography with ultraviolet detection, Food Chem. 196 (2016) 428-436. 
doi:10.1016/j.foodchem.2015.09.060.

[56] R. Kumar, Gaurav, Heena, A.K. Malik, A. Kabir, K.G. Furton, Efficient analysis of selected estrogens using fabric phase sorptive extraction and high performance liquid chromatography-fluorescence detection, J. Chromatogr. A. 1359 (2014) 16-25. doi:10.1016/j.chroma.2014.07.013.

[57] V. Samanidou, L.-D. Galanopoulos, A. Kabir, K.G. Furton, Fast extraction of amphenicols residues from raw milk using novel fabric phase sorptive extraction followed by high-performance liquid chromatography-diode array detection, Anal. Chim. Acta. 855 (2015) 41-50. doi:10.1016/j.aca.2014.11.036.

[58] K.G. Furton, A. Kabir, Novel Sol-Gel Sorbents in Sorptive Microextraction Analytical Microextraction Techniques, Anal. Microextraction Tech. (2016) 28-69.

[59] R. Guedes-Alonso, L. Ciofi, Z. Sosa-Ferrera, J.J. Santana-Rodr??guez, M. Del Bubba, A. Kabir, K.G. Furton, Determination of androgens and progestogens in environmental and biological samples using fabric phase sorptive extraction coupled to ultra-high performance liquid chromatography tandem mass spectrometry, J. Chromatogr. A. 1437 (2016) 116-126. doi:10.1016/j.chroma.2016.01.077.

[60] S.S. Lakade, F. Borrull, K.G. Furton, A. Kabir, N. Fontanals, R.M. Marcé, Comparative study of different fabric phase sorptive extraction sorbents to determine emerging contaminants from environmental water using liquid chromatography-tandem mass spectrometry, Talanta. 144 (2015) 13421351. doi:10.1016/j.talanta.2015.08.009.

[61] I. Racamonde, R. Rodil, J.B. Quintana, B.J. Sieira, A. Kabir, K.G. Furton, R. Cela, Fabric phase sorptive extraction: A new sorptive microextraction technique for the determination of non-steroidal anti-inflammatory drugs from environmental water samples, Anal. Chim. Acta. 865 (2015) 22-30. doi:10.1016/j.aca.2015.01.036.

[62] M. Roldán-Pijuán, R. Lucena, S. Cárdenas, M. Valcárcel, A. Kabir, K.G. Furton, Stir fabric phase sorptive extraction for the determination of triazine herbicides in environmental waters by liquid chromatography, $\mathrm{J}$. Chromatogr. A. 1376 (2015) 35-45. doi:10.1016/j.chroma.2014.12.027.

[63] V. Samanidou, A. Kabir, K.G. Furton, K. Michaelidou, Fabric phase sorptive extraction of selected penicillin antibiotic residues from intact milk followed by high performance liquid chromatography with diode array detection, Food Chem. 224 (2017) 131-138.

[64] S. Cardenas, M. Valcarcel, A. Kabir, K.G. Furton, M.C. Alcudia-Leon, R. Lucena, Integrated sampling and analysis unit for the determination of sexual pheromones in environmental air using fabric phase sorptive 
extraction and headspace-gas chromatography-mass spectrometry, J. Chromatogr. A. 1488 (2017) 17-25.

[65] M. Locatelli, A. Kabir, K.G. Furton, D. Innosa, T. Lopatriello, A fabric phase sorptive extraction-High performance liquid chromatography-Photo diode array detection method for the determination of twelve azole antimicrobial drug residues in human plasma and urine, J. Chromatogr. B. 1040 (2016) 192-198.

[66] J.J. Santana-Rodriguez, A. Kabir, K.G. Furton, R.B. Garcia-Guerra, S. Montesdeoca-Esponda, Z. Sosa-Ferrera, Rapid monitoring of residual UVstabilizers in seawater samples from beaches using fabric phase sorptive extraction and UHPLC-MS/MS, Chemosphere. 164 (2016) 201-207.

[67] A. Kabir, K.G. Furton, A. Malik, Innovations in sol-gel microextraction phases for solvent-free sample preparation in analytical chemistry, TrAC Trends Anal. Chem. 45 (2013) 197-218. doi:10.1016/j.trac.2012.11.014.

[68] A. Kumar, Gaurav, A.K. Malik, D.K. Tewary, B. Singh, A review on development of solid phase microextraction fibers by sol-gel methods and their applications, Anal. Chim. Acta. (2008) 1-14.

doi:10.1016/j.aca.2008.01.028.

[69] C.J. Brinker, G.W. Scherer, Sol-gel science : the physics and chemistry of sol-gel processing, Academic Press, 1990.

[70] L.C. Klein, Sol-Gel Technology for Thin Films, Fibers, Preforms, Electronics, and Specialty Shapes., First, Noyes Publications, New Jersey, 1988.

[71] L. Cai, S. Gong, M. Chen, C. Wu, Vinyl crown ether as a novel radical crosslinked sol-gel SPME fiber for determination of organophosphorus pesticides in food samples, Anal. Chim. Acta. 559 (2006) 89-96. doi:10.1016/j.aca.2005.11.048.

[72] K. Robards, P.R. Haddad, P.E. Jackson, Principles and Practice of Modern Chromatographic Methods, Academic Press, San Diego, CA, 1994.

[73] International Union of Applied Chemistry (IUPAC), Recommendations on Nomenclature for Chromatography, Pure Appl. Chem. 37 (1974) 445-462.

[74] P. Wang, V. Murugaiah, B. Yeung, P. Vouros, R.W. Giese, 2Phosphoglycolate and glycolate-electrophore detection, including detection of 87 zeptomoles of the latter by gas chromatography-electron-capture mass spectrometry, J. Chromatogr. A. 721 (1996) 289-296. doi:10.1016/0021-9673(95)00791-1.

[75] G.A. Valaskovic, N.L. Kelleher, F.W. McLafferty, Attomole Protein Characterization by Capillary Electrophoresis-Mass Spectrometry, Science (80-. ). 273 (1996). 
http://science.sciencemag.org/content/273/5279/1199.long.

[76] E. de Hoffman, V. Stroobant, Mass Spectrometry Principles and Applications, Third, John Wiley \& Sons, 2007.

[77] M. Mann, K. Meng, J.B. Fenn, Interpreting Mass Spectra of Multiply Charged lons, 61 (1989) 1702-1708.

http://pubs.acs.org/doi/pdf/10.1021/ac00190a023 (accessed June 27, 2017).

[78] M. Yamashita, J.B. Fenn, Electrospray ion source. Another variation on the free-jet theme, J. Phys. Chem. 88 (1984) 4451-4459. doi:10.1021/j150664a002.

[79] M. Yamashita, B.J. Fenn, Negative ion production with the electrospray ion source, J Phys.Chem. 88 (1984) 4671-4675. doi:10.1021/j150664a046.

[80] M.G. Ikonomou, A.T. Blades, P. Kebarle, Investigations of the Electrospray Interface for Liquid Chromatography/Mass Spectrometry, Anal. Chem. 62 (1990) 957-967. http://pubs.acs.org/doi/pdf/10.1021/ac00208a012 (accessed June 27, 2017).

[81] R.D. Smith, J.A. Loo, C.G. Edmonds, C.J. Barinaga, H.R. Udseth, PERSPECTIVE: ANALYTICAL BIOTECHNOLOGY New Developments in Biochemical Mass Spectrometry: Electrospray lonization, Anal. Chem. 62 (1990) 882-899. http://pubs.acs.org/doi/pdf/10.1021/ac00208a002 (accessed June 27, 2017).

[82] M. Mann, Electrospray: Its potential and limitations as an ionization method for biomolecules, Org. Mass Spectrom. 25 (1990) 575-587. doi:10.1002/oms.1210251104.

[83] F.M. Dunnivant, J.W. Ginsbach, Gas Chromatography, Liquid Chromatography, Capillary Electrophoresis - Mass Spectrometry-A BASIC INTRODUCTION, 2011.

[84] W. Paul, S. Helmut, Notizen: Ein neues Massenspektrometer ohne Magnetfeld, Zeitschrift Für Naturforsch. A. 8 (1953) 448-450.

[85] J. Yinon, Forensic and environmental detection of explosives, John Wiley, 1999.

[86] J. Yinon, Toxicity and Metabolism of Explosives, CRC Press, Inc., Boca Raton, 1990.

https://books.google.com/books/about/Toxicity_and_Metabolism_of_Explo sives.html?id=BD3c7FN4x5YC\&printsec=frontcover\&source=kp_read_butt on\&hl=en\#v=onepage \&q\&f=false.

[87] M. Smith, G.E. Collins, J. Wang, M icroscale solid-phase extraction system for explosives, J. Chromatogr. A. 991 (2003) 159-167. www.elsevier.com 
(accessed March 24, 2017).

[88] N.C. for B. Information, PubChem Compound Database CID=7434, n.d. https://pubchem.ncbi.nlm.nih.gov/compound/7434.

[89] EPA, Technical Fact Sheet - ( NDMA ) At a Glance, 2012.

[90] N.C. for B. Information, PubChem Compound Database CID=7452, n.d. https://pubchem.ncbi.nlm.nih.gov/compound/7452.

[91] N.C. for B. Information, PubChem Compound Database CID=6944, n.d. https://pubchem.ncbi.nlm.nih.gov/compound/6944.

[92] EPA, Nitrobenzene, 2000.

[93] N.C. for B. Information, PubChem Compound Database CID=7473, n.d. https://pubchem.ncbi.nlm.nih.gov/compound/7473.

[94] EPA, Technical Fact Sheet - 2,4,6-Trinitrotoluene (TNT), 2014. http://www2.epa.gov/sites/production/files/201403/documents/ffrrofactsheet_contaminant_tnt_january2014_final.pdf.

[95] N.C. for B. Information, PubChem Compound Database CID=7422, n.d. https://pubchem.ncbi.nlm.nih.gov/compound/7422.

[96] N.C. for B. Information, PubChem Compound Database CID=10178, n.d. https://pubchem.ncbi.nlm.nih.gov/compound/10178.

[97] J.-D. Berset, U. Ochsenbein, M. Zeh, Comparing solid phase extraction and direct injection for the analysis of ultra-trace levels of relevant explosives in lake water and tributaries using liquid chromatographyelectrospray tandem mass spectrometry, Chemosphere. 72 (2008) 974980.

[98] D. Bernet, T. Wahli, C. Kueng, H. Segner, Frequent and unexplained gonadal abnormalities in whitefish (central alpine Coregonus sp.) from an alpine oligotrophic lake in Switzerland, Dis. Aquat. Org. Dis Aquat Org. 61 (2004) 137-148.

[99] N.C. for B. Information, PubChem Compound Database CID=241, n.d. https://pubchem.ncbi.nlm.nih.gov/compound/241.

[100] N.C. for B. Information, PubChem Compound Database;CID=7237, n.d. https://pubchem.ncbi.nlm.nih.gov/compound/7237.

[101] N.C. for B. Information, PubChem Compound Database CID=1140, n.d. https://pubchem.ncbi.nlm.nih.gov/compound/1140.

[102] N.C. for B. Information, PubChem Compound Database CID=7809, n.d. https://pubchem.ncbi.nlm.nih.gov/compound/7809.

[103] N.C. for B. Information, PubChem Compound Database CID=7500, n.d. 
https://pubchem.ncbi.nlm.nih.gov/compound/7500.

[104] S. Camou, E. Tamechika, T. Horiuchi, Portable sensor for determining benzene concentration from airborne/ liquid samples with high accuracy, NTT Tech. Rev. 10 (2012) 10-12.

[105] R. Atkinson, Atmospheric chemistry of VOCs and NOx, Atmos. Environ. 34 (2000) 2063-2101. http://ac.els-

cdn.com.ezproxy.fiu.edu/S1352231099004604/1-s2.0-

S1352231099004604-main.pdf? tid=24e99c94-5f96-11e7-83fd00000aab0f01\&acdnat=1499048592_043da7c102e5e9f27866fdf804703ac 1 (accessed July 2, 2017).

[106] D.L. Heavner, W.T. Morgan, M.W. Ogden, Determination of volatile organic compounds and ETS apportionment in 49 homes, Environ. Int. 21 (1995) 3-21. doi:10.1016/0160-4120(94)00018-3.

[107] A. Allouch, S. Le calve, C.A. Serra, Portable, miniature, fast and high sensitive real-time analyzers: BTEX detection, Sensors Actuators B Chem. 182 (2013) 446-452. http://ac.elscdn.com.ezproxy.fiu.edu/S0925400513002918/1-s2.0S0925400513002918-main.pdf?_tid=9864700c-5f93-11e7-8b3100000aabOf6b\&acdnat $=1499047498 \_511 d 840647$ c549f129fda787253406 59.

[108] Q. Li, X. Xu, L.F. Sen-Chun, X. Wang, Determination of trace PAHs in seawater and sediment pore-water by solid-phase microextraction (SPME) coupled with GC/MS, Sci. China Ser. B Chem. 49 (2006) 481-491. doi:10.1007/s11426-006-2026-5.

[109] Z. Dang, Z. Lin, H. Yin, G. Lu, X. Yi, D. Peng, L.Y. Wick, S. Liu, C. Liao, C. Guo, X. Liang, Drivers and applications of integrated clean-up technologies for surfactant-enhanced remediation of environments contaminated with polycyclic aromatic hydrocarbons (PAHs), Environ. Pollut. 225 (2017) 129140.

[110] F. Coulon, K. Jones, H. Li, Q. Hu, J. Gao, F. Li, M. Chen, Y.-G. Zhu, R. Liu, M. Liu, K. Canning, N. Harries, P. Bardos, P. Nathanail, R. Sweeney, D. Middleton, M. Charnley, J. Randall, M. Richell, T. Howard, I. Martin, S. Spooner, J. Weeks, M. Cave, F. Yu, F. Zhang, Y. Jiang, P. Longhurst, G. Prpich, R. Bewley, J. Abra, S. Pollard, China's soil and groundwater management challenges: Lessons from the UK's experience and opportunities for China, Environ. Int. 91 (2016) 196-200. doi:10.1016/j.envint.2016.02.023.

[111] M.S. Garc, ! la-Falc, ! On, B. Cancho-Grande, J. Simal-G, ! Andara, Stirring bar sorptive extraction in the determination of PAHs in drinking waters, Water Res. 38 (2004) 1679-1684. doi:10.1016/j.watres.2003.12.034. 
[112] N.C. for B. Information, PubChem Compound Database CID=931, n.d. https://pubchem.ncbi.nlm.nih.gov/compound/931.

[113] N.C. for B. Information, PubChem Compound Database CID=8418, n.d. https://pubchem.ncbi.nlm.nih.gov/compound/8418.

[114] N.C. for B. Information, PubChem Compound Database CID=6853, n.d. https://pubchem.ncbi.nlm.nih.gov/compound/6853.

[115] N.C. for B. Information, PubChem Compound Database CID=9154, n.d. https://pubchem.ncbi.nlm.nih.gov/compound/9154.

[116] N.C. for B. Information, PubChem Compound Database CID=995, n.d. https://pubchem.ncbi.nlm.nih.gov/compound/995.

[117] S.K. Brar, T. Kadri, T. Rouissi, M. Cledon, S. Sarma, M. Verma, Biodegradation of polycyclic aromatic hydrocarbons (PAHs) by fungal enzymes: A review, J. Environ. Sci. 51 (2017) 52-74. http://ac.elscdn.com.ezproxy.fiu.edu/S1001074216306167/1-s2.0S1001074216306167-main.pdf?_tid=e9b866f6-60ca-11e7-aa3a00000aacb361\&acdnat=1499181208_fd814012911eb009f7ff8c86a3a1d59 a.

[118] C. Hansch, A. Leo, D.H. Hoekman, Exploring QSAR.: Hydrophobic, electronic, and steric constants, American Chemical Society, 1995.

[119] G. Kaklamanos, G. Theodoridis, Rapid multi-method for the determination of growth promoters in bovine milk by liquid chromatography-tandem mass spectrometry, J. Chromatogr. B. 930 (2013) 22-29. doi:10.1016/j.jchromb.2013.04.013.

[120] Y. Juan-Garcia, Ana; Font, Guillermina; Pico, Determination of quinolone residues in chicken and fish by capillary electrophoresis-mass spectrometry, Electrophoresis. 27 (2006) 2240-2249.

[121] V. Samanidou, E. Evaggelopoulou, M. Trötzmüller, X. Guo, E. Lankmayr, Multi-residue determination of seven quinolones antibiotics in gilthead seabream using liquid chromatography-tandem mass spectrometry, J. Chromatogr. A. 1203 (2008) 115-123. doi:10.1016/j.chroma.2008.07.003.

[122] X. Peng, Z. Wang, C. Yang, F. Chen, B. Mai, Simultaneous determination of endocrine-disrupting phenols and steroid estrogens in sediment by gas chromatography-mass spectrometry, J. Chromatogr. A. 1116 (2006) 5156. doi:10.1016/j.chroma.2006.03.017.

[123] EPA, Bisphenol A (BPA) Action Plan, (n.d.). https://www.epa.gov/assessing-and-managing-chemicals-undertsca/bisphenol-bpa-action-plan.

[124] J.R. Rochester, Bisphenol A and human health: A review of the literature, 
Reprod. Toxicol. 42 (2013) 132-155. doi:10.1016/j.reprotox.2013.08.008.

[125] P.S. Bailin, M. with Margaret Byrne, S. Lewis, R. Liroff, Public Awareness Drives Market for Safer Alternatives Bisphenol A Market Analysis Report, (2008).

[126] L.N. Vandenberg, T. Colborn, T.B. Hayes, J.J. Heindel, D.R. Jacobs, D.-H. Lee, T. Shioda, A.M. Soto, F.S. vom Saal, W. V Welshons, R.T. Zoeller, J.P. Myers, J.P. Myers, Hormones and endocrine-disrupting chemicals: low-dose effects and nonmonotonic dose responses., Endocr. Rev. 33 (2012) 378-455. doi:10.1210/er.2011-1050.

[127] P.A. Fowler, M. Bellingham, K.D. Sinclair, N.P. Evans, P. Pocar, B. Fischer, K. Schaedlich, J.-S. Schmidt, M.R. Amezaga, S. Bhattacharya, S.M. Rhind, P.J. O'Shaughnessy, Impact of endocrine-disrupting compounds (EDCs) on female reproductive health, Mol. Cell. Endocrinol. 355 (2012) 231-239. doi:10.1016/j.mce.2011.10.021.

[128] L. Wang, Y.-Q. Cai, B. He, C.-G. Yuan, D.-Z. Shen, J. Shao, G.-B. Jiang, Determination of estrogens in water by HPLC-UV using cloud point extraction, Talanta. 70 (2006) 47-51. doi:10.1016/j.talanta.2006.01.013.

[129] N.E. Skakkebaek, E. Rajpert-De Meyts, K.M. Main, Testicular dysgenesis syndrome: an increasingly common developmental disorder with environmental aspects., Hum. Reprod. 16 (2001) 972-8. http://www.ncbi.nlm.nih.gov/pubmed/11331648 (accessed February 27, 2017).

[130] T.K. Woodruff, C.L. Walker, Fetal and early postnatal environmental exposures and reproductive health effects in the female, Fertil. Steril. 89 (2008) e47-e51. doi:10.1016/j.fertnstert.2007.12.029.

[131] EPA, EPA Chemistry Dashboard Hexestrol, (n.d.). https://comptox.epa.gov/dashboard/DTXSID2022381.

[132] J.D. Hayes, A. Malik, C. Shende, A. Kabir, E. Townsend, D. Wang, S.L. Chong, L. Fang, S. Kulkarni, K. Alhooshani, S. Florida, E.F. Avenue, SolGel Column Technology for Single-Step Deactivation, Coating , and Stationary-Phase Immobilization in High-Resolution Capillary Gas Chromatography, Anal. Chem. 79 (1997) 9441-9451. doi:10.1021/ac970614v.

[133] nanoScience, Scanning Electron Microscopy, (n.d.). http://dev.nanoscience.com/technology/sem-technology/how-sem-works/.

[134] J. Schweitzer, Scanning Electron Microscope, (2014). https://www.purdue.edu/ehps/rem/rs/sem.htm.

[135] M. Bradley, FTIR Basics, (n.d.). https://www.thermofisher.com/us/en/home/industrial/spectroscopy- 
elemental-isotope-analysis/spectroscopy-elemental-isotope-analysislearning-center/molecular-spectroscopy-information/ftir-information/ftirbasics.html.

[136] S. Kuche Loghmani, M. Farrokhi-Rad, T. Shahrabi, Effect of polyethylene glycol on the electrophoretic deposition of hydroxyapatite nanoparticles in isopropanol, Ceram. Int. 39 (2013) 7043-7051.

doi:10.1016/j.ceramint.2013.02.043.

[137] Agilent Technologies Inc., Evaluating System Suitability CE, GC, LC and A/D ChemStation, (2004) 1-17.

[138] Gaurav, A.K. Malik, P.K. Rai, Development of a new SPME-HPLC-UV method for the analysis of nitro explosives on reverse phase amide column and application to analysis of aqueous samples, J. Hazard. Mater. 172 (2009) 1652-1658. doi:10.1016/j.jhazmat.2009.08.039.

[139] E.M. Reyes-Gallardo, G. Lasarte-Aragon??s, R. Lucena, S. C??rdenas, M. Valc??rcel, Hybridization of commercial polymeric microparticles and magnetic nanoparticles for the dispersive micro-solid phase extraction of nitroaromatic hydrocarbons from water, J. Chromatogr. A. 1271 (2013) 5055. doi:10.1016/j.chroma.2012.11.040.

[140] M.B. Gholivand, M. Shamsipur, M. Shamizadeh, R. Moradian, B. Astinchap, Cobalt oxide nanoparticles as a novel high-efficiency fiber coating for solid phase microextraction of benzene, toluene, ethylbenzene and xylene from aqueous solutions, Anal. Chim. Acta. 822 (2014) 30-36. doi:10.1016/j.aca.2014.02.032.

[141] H. Bagheri, A. Mir, E. Babanezhad, An electropolymerized aniline-based fiber coating for solid phase microextraction of phenols from water, Anal. Chim. Acta. 532 (2005) 89-95. doi:10.1016/j.aca.2004.10.040.

[142] Y. Zhang, D. Wu, X. Yan, Y. Guan, Rapid solid-phase microextraction of polycyclic aromatic hydrocarbons in water samples by a coated throughpore sintered titanium disk, Talanta. 154 (2016) 400-408. doi:10.1016/j.talanta.2016.03.094.

[143] M. Bourdat-Deschamps, J.-J. Daudin, E. Barriuso, An experimental design approach to optimise the determination of polycyclic aromatic hydrocarbons from rainfall water using stir bar sorptive extraction and high performance liquid chromatography-fluorescence detection., J. Chromatogr. A. 1167 (2007) 143-53. doi:10.1016/j.chroma.2007.08.025.

[144] J.L. Zhou, A.J. King, J.W. Readman, Determination of polycyclic aromatic hydrocarbons in water by solid-phase microextraction-gas chromatography-mass spectrometry, Anal. Chim. Acta. 523 (2004) 259267. http://ac.els-cdn.com.ezproxy.fiu.edu/S0003267004009377/1-s2.0S0003267004009377-main.pdf?_tid=347ed9d2-7cae-11e7-9f4e- 
00000aab0f01\&acdnat=1502247510_9db2c0f3533a485d11d3ca49748c22 a2.

[145] M.-C. Wei, J.-F. Jen, Determination of polycyclic aromatic hydrocarbons in aqueous samples by microwave assisted headspace solid-phase microextraction and gas chromatography/flame ionization detection, Talanta. 72 (2007) 1269-1274. doi:10.1016/j.talanta.2007.01.017.

[146] V. Fernández-González, E. Concha-Graña, S. Muniategui-Lorenzo, P. López-Mahía, D. Prada-Rodríguez, Solid-phase microextraction-gas chromatographic-tandem mass spectrometric analysis of polycyclic aromatic hydrocarbons Towards the European Union water directive 2006/0129 EC, J. Chromatogr. A. 1176 (2007) 48-56. doi:10.1016/j.chroma.2007.11.006.

[147] J. Feng, M. Sun, J. Li, L. Xu, X. Liu, S. Jiang, Polydopamine supported preparation method for solid-phase microextraction coatings on stainless steel wire, J. Chromatogr. A. 1218 (2011) 3601-3607. doi:10.1016/j.chroma.2011.04.018.

[148] X. Yan, D. Wu, H. Peng, K. Ding, C. Duan, Y. Guan, Array capillary in-tube solid-phase microextraction: A rapid preparation technique for water samples, J. Chromatogr. A. 1244 (2012) 69-76. doi:10.1016/j.chroma.2012.05.015.

[149] X. Mao, B. Hu, M. He, W. Fan, Stir bar sorptive extraction approaches with a home-made portable electric stirrer for the analysis of polycyclic aromatic hydrocarbon compounds in environmental water, J. Chromatogr. A. 1260 (2012) 16-24. doi:10.1016/j.chroma.2012.08.062.

[150] J. Gañán, S. Morante-Zarcero, D. Pérez-Quintanilla, M.L. Marina, I. Sierra, One-pot synthesized functionalized mesoporous silica as a reversed-phase sorbent for solid-phase extraction of endocrine disrupting compounds in milks, J. Chromatogr. A. 1428 (2016) 228-235. doi:10.1016/j.chroma.2015.08.063.

[151] B. Socas-Rodríguez, M. Asensio-Ramos, J. Hernández-Borges, M.Á. Rodríguez-Delgado, Hollow-fiber liquid-phase microextraction for the determination of natural and synthetic estrogens in milk samples, J. Chromatogr. A. 1313 (2013) 175-184. doi:10.1016/j.chroma.2013.05.028.

[152] R. Rodríguez-Gómez, A. Zafra-Gómez, F.J. Camino-Sánchez, O. Ballesteros, A. Navalón, Gas chromatography and ultra high performance liquid chromatography tandem mass spectrometry methods for the determination of selected endocrine disrupting chemicals in human breast milk after stir-bar sorptive extraction, J. Chromatogr. A. 1349 (2014) 69-79. doi:10.1016/j.chroma.2014.04.100. 
VITA

RODOLFO MESA

2012

B.S., Chemistry Florida International University Miami, Florida

2016

M.S. Forensic Science

Florida International University

Miami, Florida

$2016-2017$

Doctoral Candidate

Florida International University

Miami, Florida

Teaching Assistant

Florida International University

Miami, Florida

Featured on a Miami Herald article for graduate research projects with Fabric Phase Sorptive Extraction

Presenter of FPSE advancements and future possibilities on behalf of FIU at eMerge Americas 2017

Analytical chemistry method development, validation, application and troubleshooting both within sample preparation and HPLCbased instrumental analysis

Literature research within peer-reviewed journals in chemistry and regulatory agency publications

\section{PUBLICATIONS AND PRESENTATIONS:}

1. A. Kabir, R. Mesa, J. L. Jurmain, K. G. Furton. Fabric Phase Sorptive Extraction, Explained. Journal: Separations, 2017, 4, 21.

2. R. Mesa, A. Kabir, K. G. Furton. Direct Ultra-Trace Analysis of Estrogenic Endocrine Disrupting Chemicals and Bisphenol A from Whole Milk with Fabric Phase Sorptive Extraction. Journal of Chromatography A (pending) 
1. Rodolfo Mesa, Abuzar Kabir, Kenneth G. Furton; Analysis of Trace Organics at Parts per Trillion Level Concentrations Using a Novel Sample Preparation Technique: Fabric Phase Sorptive Extraction; AAFS Annual Meeting; 02/19/2014; Seattle, WA

2. Rodolfo Mesa, Abuzar Kabir, Kenneth G. Furton; Analysis of Trace Organics at Parts per Trillion Level Concentrations Using a Novel Sample Preparation Technique: Fabric Phase Sorptive Extraction; IFRI Symposium; 04/30/2014; Miami, FL

3. Rodolfo Mesa, Abuzar Kabir, Kenneth G. Furton; Trace Analysis of Nitroaromatic Explosives Using a Novel Sample Preparation Technique: Fabric Phase Sorptive Extraction; FAME; 05/10/2014; Palm Harbor, FL

4. Rodolfo Mesa, Abuzar Kabir, Kenneth G. Furton; Fabric Phase Sorptive Extraction Media: A Highly Effective Forensic Sample Collection and Storage Device; AAFS Annual Meeting; 02/21/2015; Orlando, FL

5. Rodolfo Mesa, Abuzar Kabir, Kenneth G. Furton; Fabric Phase Sorptive Extraction Media: A Forensic Sample Collection and Storage Device; IFRI Symposium; 05/05/2015; Miami, FL

6. Rodolfo Mesa, Manuel Bode, Rayma Blanco, Abuzar Kabir, Kenneth G. Furton; Trace Analysis of Endocrine Disrupting Chemicals in Whole Milk Using Fabric Phase Sorptive Extraction; FAME; 05/09/2015; Palm Harbor, FL

7. Rodolfo Mesa, Abuzar Kabir, Kenneth G. Furton; Direct Ultra-Trace Analysis of Selected Estrogenic Endocrine Disruptors Including Bisphenol A from Whole Milk Using Fabric Phase Sorptive Extraction; IFRI Symposium; 03/15/2016; Miami, FL

8. Rodolfo Mesa, Abuzar Kabir, Kenneth G. Furton; Fabric Phase Sorptive Extraction Media: A Highly Effective and Versatile Storage Device; FAME; 05/07/2016; Palm Harbor, FL

9. Rodolfo Mesa, Abuzar Kabir, Kenneth G. Furton; Capsule Phase Microextraction (CPME): A New and Powerful Arsenal in Analytical and Forensic Sample Preparation; AAFS Annual Meeting; 02/15/2017; New Orleans, LA 